\title{
Rehabilitation of deteriorated timber piles with fiber reinforced composite wrap systems
}

John William Harper

Follow this and additional works at: https://researchrepository.wvu.edu/etd

\section{Recommended Citation}

Harper, John William, "Rehabilitation of deteriorated timber piles with fiber reinforced composite wrap systems" (2017). Graduate Theses, Dissertations, and Problem Reports. 3998.

https://researchrepository.wvu.edu/etd/3998

This Problem/Project Report is protected by copyright and/or related rights. It has been brought to you by the The Research Repository @WVU with permission from the rights-holder(s). You are free to use this Problem/Project Report in any way that is permitted by the copyright and related rights legislation that applies to your use. For other uses you must obtain permission from the rights-holder(s) directly, unless additional rights are indicated by a Creative Commons license in the record and/ or on the work itself. This Problem/Project Report has been accepted for inclusion in WVU Graduate Theses, Dissertations, and Problem Reports collection by an authorized administrator of The Research Repository @ WVU. For more information, please contact researchrepository@mail.wvu.edu. 


\title{
Rehabilitation of Deteriorated Timber Piles with Fiber Reinforced Composite Wrap Systems
}

\author{
John William Harper
}

Problem Report submitted to the

College of Engineering and Mineral Resources at

West Virginia University in

partial fulfillment of the requirements

for the degree of

\section{Master of Science \\ in}

Civil Engineering

\author{
Approved by \\ Hota GangaRoa, Ph.D., Chair \\ Udaya Halabe, Ph.D. \\ Mark Skidmore, P.E.
}

\section{Department of Civil and Environmental Engineering}

\author{
Morgantown, West Virginia
}

2017

Keywords: Fiber Reinforced Polymer Composites, FRP wraps, Timber Rehabilitation 


\title{
ABSTRACT \\ Rehabilitation of Deteriorated Timber Piles with Fiber Reinforced Composite Wrap Systems
}

\author{
John William Harper \\ Constructed Facilities Center, West Virginia University
}

Fiber reinforced polymer (FRP) composite wraps have been used for timber pile repair, but there is a lack of empirical data for the development of design guidelines and strengthening equations. To address this need, this study evaluated both the bond and compressive strength of four FRP wrap systems on whole timber piles. Wrap systems evaluated used glass fabric with epoxy, polyurethane, and phenol formaldehyde resins.

Bond strengths were evaluated through push-out and pull-off bond testing data on new (unused) treated timber. The push-out test evaluated the bond strength of the wraps on timber by applying axial loads on wrapped (6" and 12" bond lengths) timber samples until bond slippage occurred. Modified pull-off tests ASTM D7522, (FRP wraps on concrete substrates), were conducted to establish pull-off bond strengths. Axial compression tests were performed on hand layup shells with varying numbers of wrap until failure. To evaluate bond strength and compressive capacity simultaneously, simulations of timber pile rehabilitation were performed also. For the simulations, two portions of timber separated by a gap (to simulate decayed timber with near zero strength) were wrapped and tested in axial compression until failure.

Results revealed that while 12" bond lengths provided a higher capacity than 6" bond lengths, their bond strengths (P/A) were reduced suggesting a non-linear relationship between bond strength and bond length. Epoxy and phenol formaldehyde resin systems predominately displayed timber failure whereas the polyurethane system failed in bond. Systems that utilized slow cure, low-viscosity resins developed high bond strengths, suggesting good timber penetration. Compression evaluations showed additional wrap layers increased the compression capacity of the shells. Some shells developed bending moments from unintended eccentric loading which reduced compressive capacity. Epoxy and phenol formaldehyde systems failed in the fibers while the polyurethane system failed due to delamination. Systems with high fiber volume fractions in the axial direction displayed the highest axial capacity. Compressive strength results corresponded well with values predicted by mechanics based FRP design equations. Since no current models for FRP bond strengths on timber are available, these results will greatly aid in their development. 


\section{ACKNOWLEDGEMENTS}

I would like to extend much gratitude to Dr. Hota GangaRao for his support and guidance during my time at CFC. His guidance and wisdom were invaluable not only in completing this work, but also in developing critical thinking and a deep appreciation for engineering and research. I would also like to thank Mr. Mark Skidmore for his encouragement and constant support in overcoming the various challenges presented in this work. Without his mentorship and assistance, this work would not have been completed. I will miss our conversations and discussions when I am gone. Gratidtude is given to Dr. Halabe for serving on my committee and providing insight into this work as well. Much thanks goes to Dr. Liang for kindly guiding me in my other projects and for providing valuable insights into the nuances involved in research. I would like to also acknowledge Mr. Jerry Nestor for his assistance and creativity throughout my time here. His energy and problem solving skills were responsible for much of the completed work and his company will be deeply missed. Thanks is also extended to the various professors and staff at WVU who assisted me throughout my time here.

Special thanks to my engineering professors at LeTourneau University for preparing me well for the rigors of graduate school. Specifically, I would like to thanks Dr. Stephen Ayers and Dr. David Dittenber for guiding me, praying for me, and encouraging me throughout this experience. Thanks is also extended to my fellow graduate students. Among these, Ben Imes, Praveen Majjigapu, Amir Houshmandyar, Jeffery Lorimer, Maria Martinez de Lahidalga, and Lupe Leon were most encouraging and helpful through my time here. I would also like to thank my Crossroads Church family for supporting and providing encouragement. Thanks also goes out to my best friend Jared Tompkins for his encouragement and conversations. His moral support was much appreciated and precious to me. I would also like thank my girlfriend Bettina Burns for her love, support, and understanding. Her encouragement and care for me was invaluable and I am incredibly thankful for her. Above all of these, my parents Don and Kim and my siblings require the most thanks. From the late night phone calls to the visits, their love and support got me through this experience. Finally I would like to give glory to God and my savior Jesus Christ. I pray that my actions and accomplishments here were done for His glory and praise above all else.

The work completed here was sponsored by the Louisiana Transportation Research Center. Much thanks to Creative Pultrusions for graciously assisting the work with the use of their invaluable equipment. 


\section{TABLE OF CONTENTS}

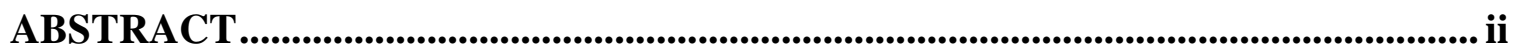

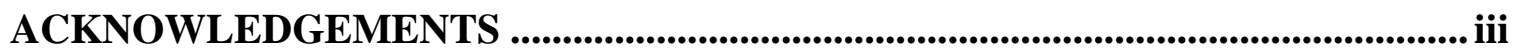

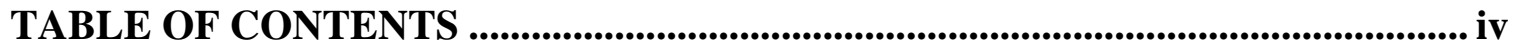

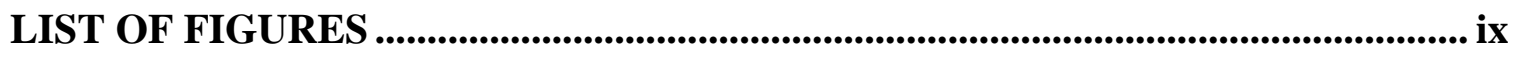

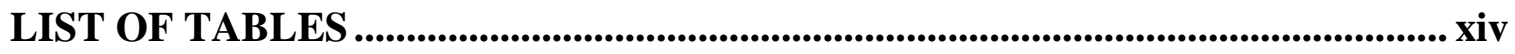

CHAPTER 1 INTRODUCTION

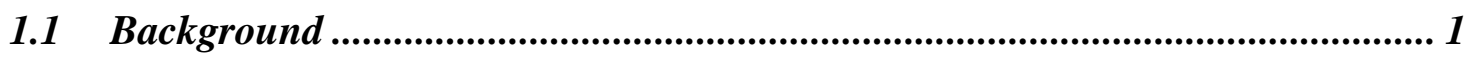

1.2 Objectives ....................................................................................................... 2

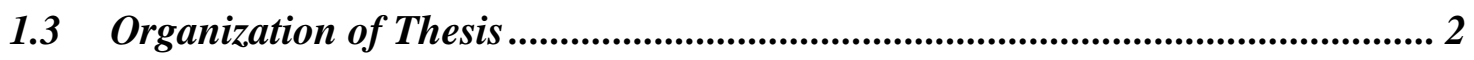

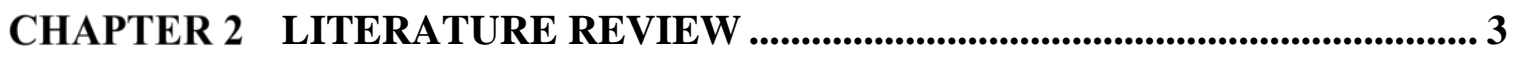

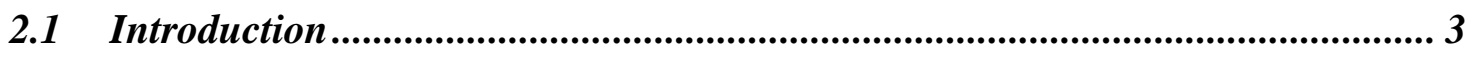

2.2 Deterioration Mechanisms of Timber Piles ...................................................... 3

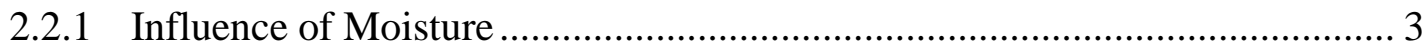

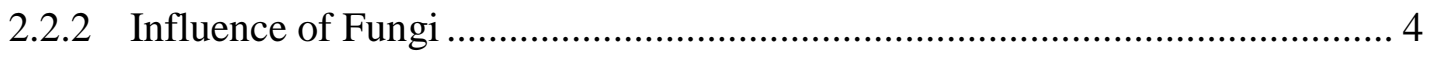

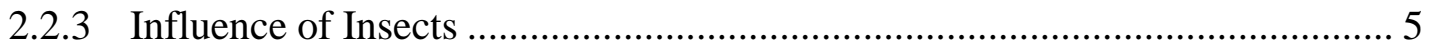

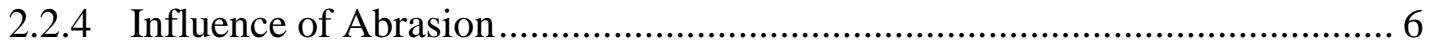

2.3 Preservative Treatments of Timber Piles...................................................... 6 
2.3.1 Oil Based Preservatives..................................................................... 6

2.3.2 Water Based Preservatives ..................................................................... 7

2.4 Rehabilitation Techniques for Timber Piles .................................................. 7

2.4.1 Concrete Jacketing (Concrete Confinement) .......................................... 7

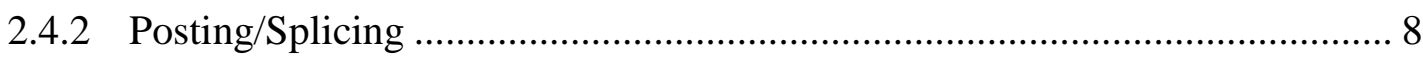

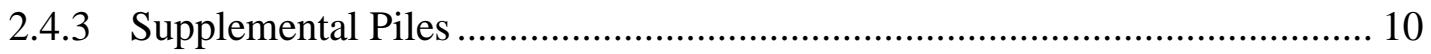

2.4.4 Prefabricated FRP Composites Shells with Grout .................................. 10

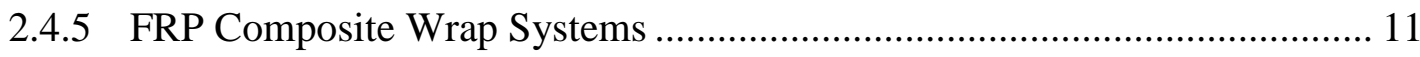

2.4.6 Summary of Rehabilitation Techniques ............................................... 13

2.5 Studies of FRP Composite Wraps on Timber Piles ...................................... 13

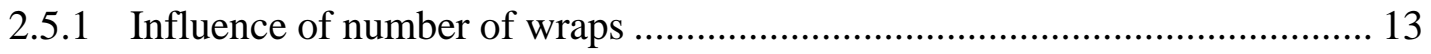

2.5.2 Common systems utilized for service conditions ................................. 15

2.6 FRP Wrap Design for Timber Pile Repair ............................................... 17

2.6.1 Basics Theory of FRP Wrap Design .................................................. 17

2.6.2 Published Design Codes for FRP wraps for Timber Piles ......................... 18

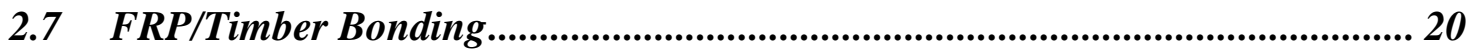

2.7.1 Preparation Factors Influencing Bond............................................... 20

2.7.2 Primers/Coupling Agents Influence on Bond with Treated Timber ............ 21

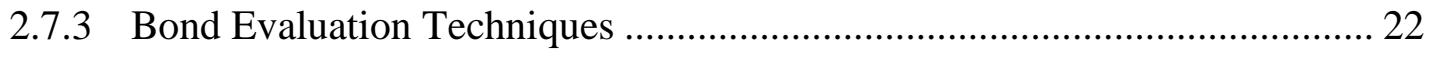

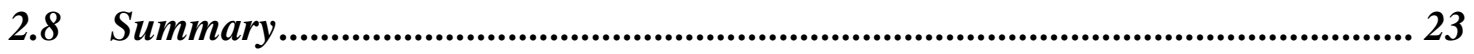




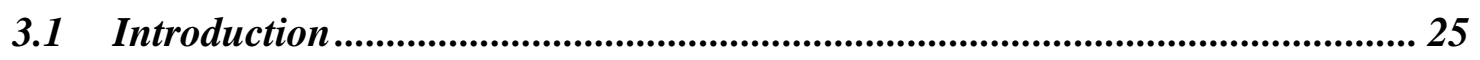

3.1.1 Limitations in current research............................................................. 25

3.1.2 Failure modes of FRP wraps on timber piles ............................................ 25

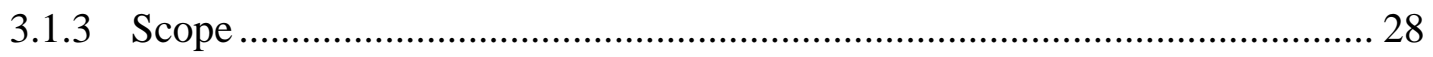

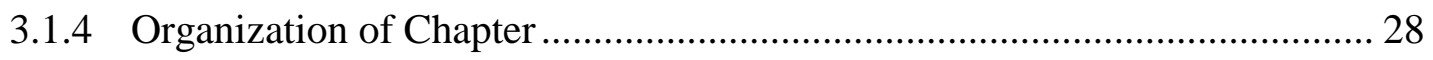

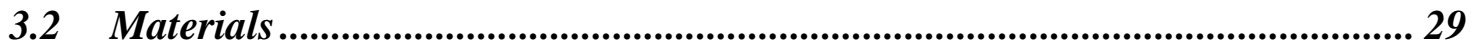

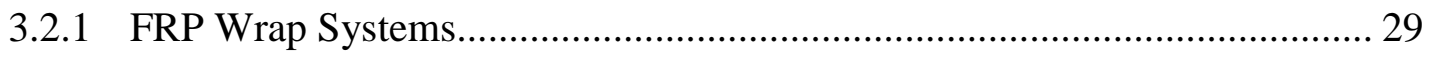

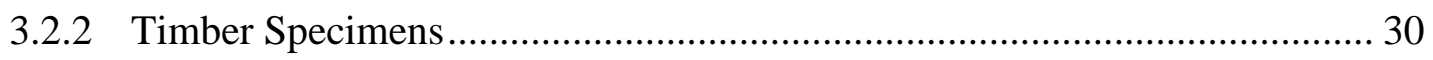

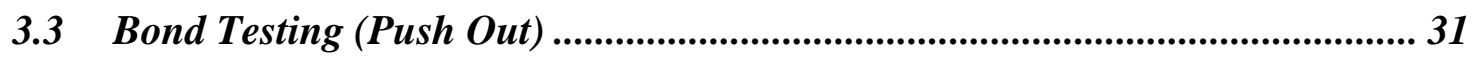

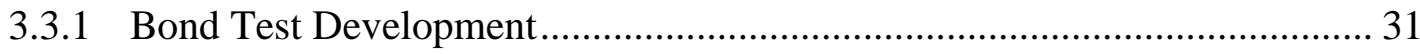

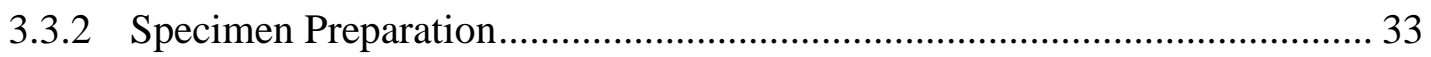

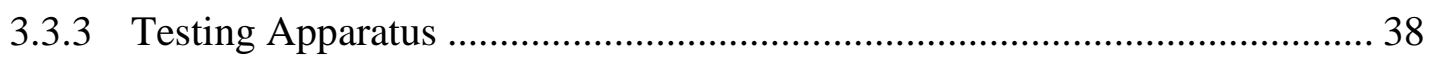

$3.4 \quad$ Pull off Bond Test (Modified ASTM D7522) ....................................................... 40

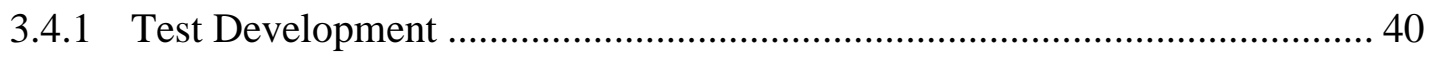

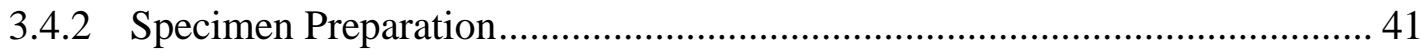

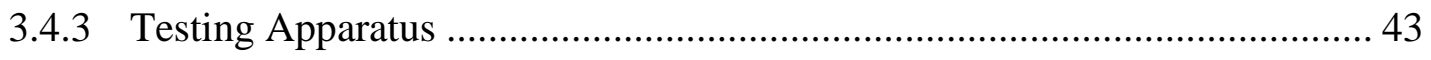

$3.5 \quad$ Compression Testing ................................................................................... 44

3.5.1 Compression Test Development ………………................................... 44

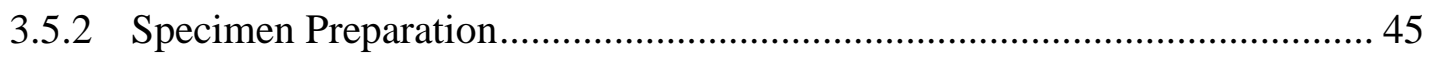




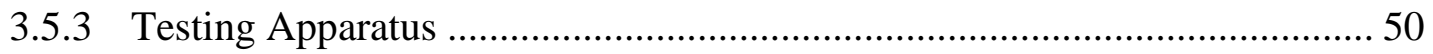

3.6 Full Scale Rehabilitation Simulation ........................................................... 52

3.6.1 Rehabilitation Simulation Test Development ........................................... 52

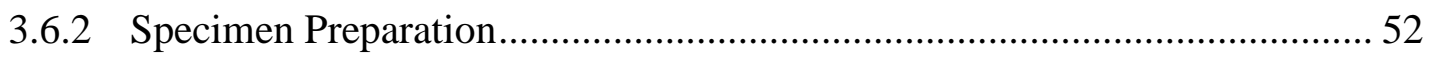

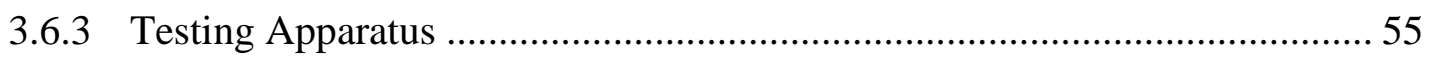

CHAPTER 4 DATA ANALYSIS and Discussion ................................................ 57

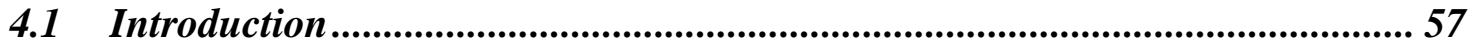

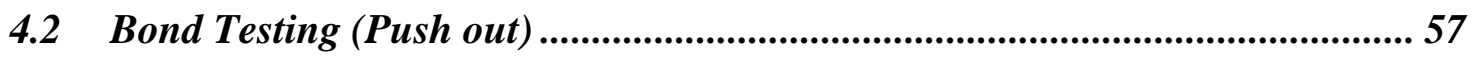

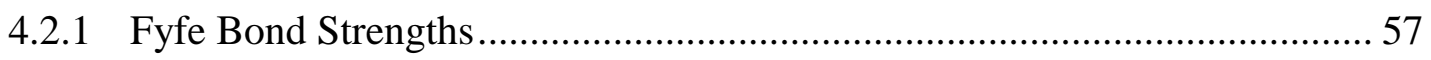

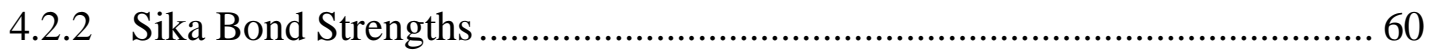

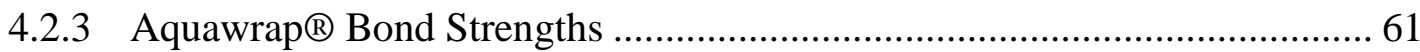

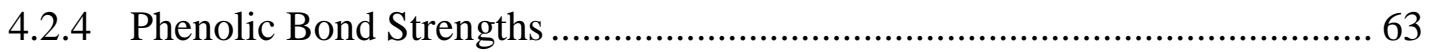

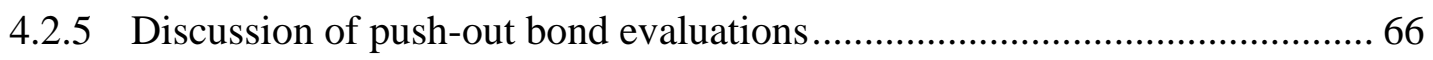

$4.3 \quad$ Pull off Bond Testing (Modified ASTM D7522)..................................... 75

4.3.1 Pull Off Bond Strength of Fyfe ......................................................... 75

4.3.2 Pull Off Bond Strength of Sika .......................................................... 76

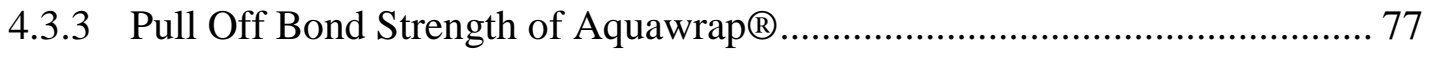

4.3.4 Pull Off Bond Strength of Phenolic .................................................... 78

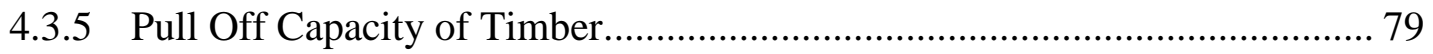

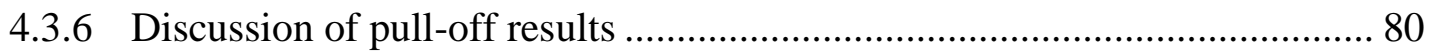




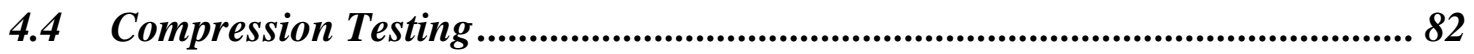

4.4.1 Fyfe Compression Results .................................................................. 83

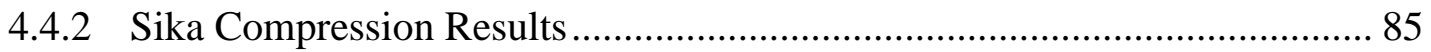

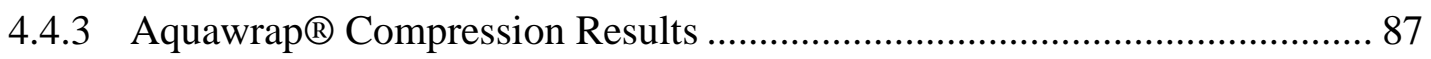

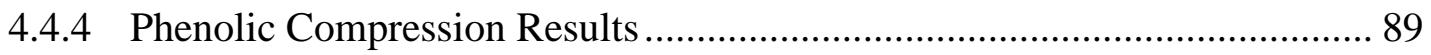

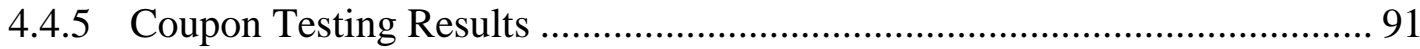

4.4.6 Discussion of Compression Results ........................................................ 96

$4.5 \quad$ Full Scale Rehabilitation Simulation ............................................................. 109

4.5.1 Summary of rehabilitation results ....................................................... 110

4.5.2 Discussion of full scale rehabilitation simulations results ....................... 112

CHAPTER 5 CONCLUSIONS AND RECOMMENDATIONS .......................... 115

5.1 Bond Strength Evaluations .................................................................. 115

5.2 Compressive Strength Evaluations....................................................... 115

5.3 Recommendations ........................................................................... 116

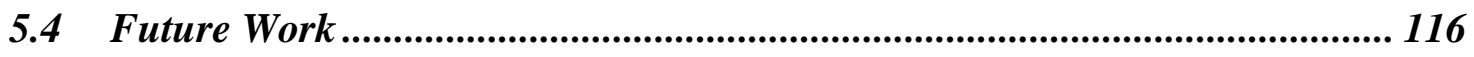

REFERENCES............................................................................................................................. 118

APPENDIX A - Bond Test Development .......................................................... 124

APPENDIX B - Compression Test Development ................................................. 128 


\section{LIST OF FIGURES}

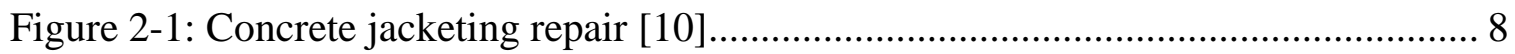

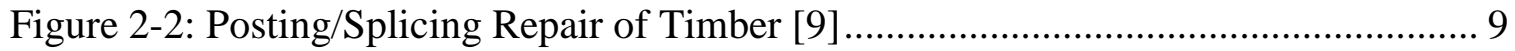

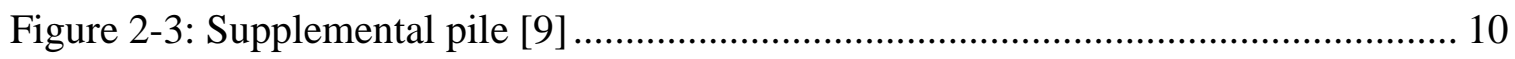

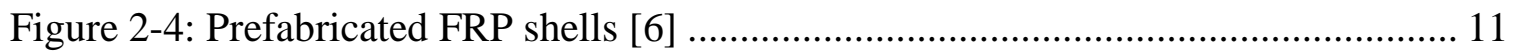

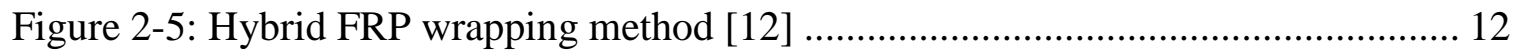

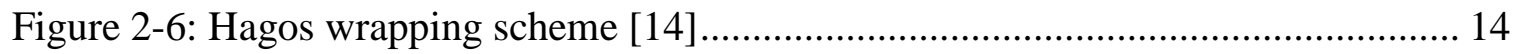

Figure 2-7: Testing schematic of apparatus used by Lopez-Anido [39]........................ 23

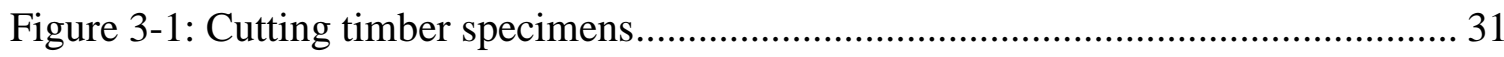

Figure 3-2: Testing schematic of pushout testing portions...................................... 32

Figure 3-3: Unwrapped timber bond specimen .................................................. 34

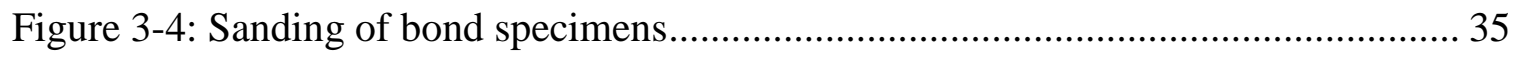

Figure 3-5: Apply primer to smaller mock up sample ............................................ 36

Figure 3-6: Apply resin to wraps for smaller mock up sample.................................. 36

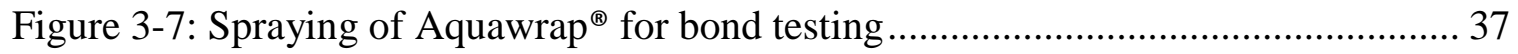

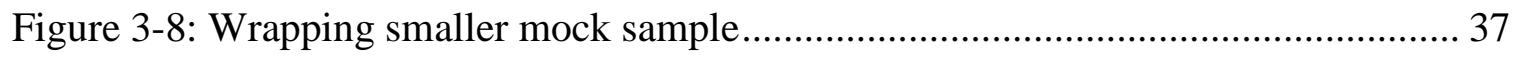

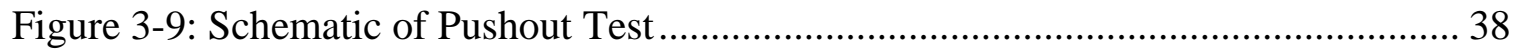

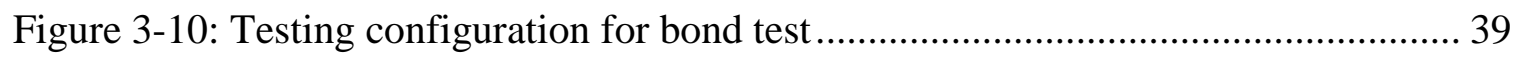

Figure 3-11: Example of pushout load vs deflection plot......................................... 40

Figure 3-12: Example of deflection over time for pushout tests .................................. 40

Figure $3-13$ : Cutting of timber for pull off tests ................................................... 42

Figure 3-14: Pull off puck attachment technique .................................................. 43

Figure 3-15: Dyna Proceq Z16 pull off tester ....................................................... 44 


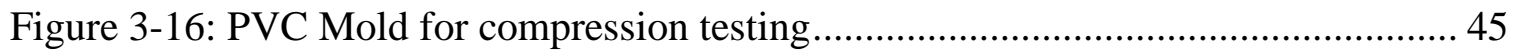

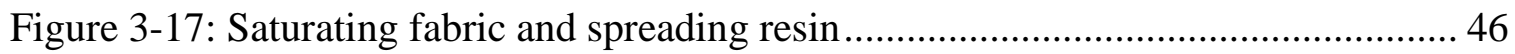

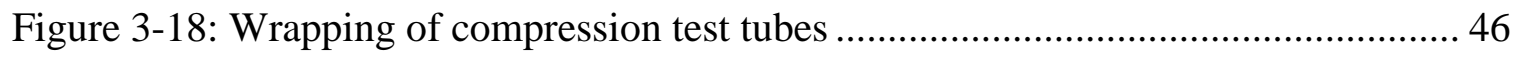

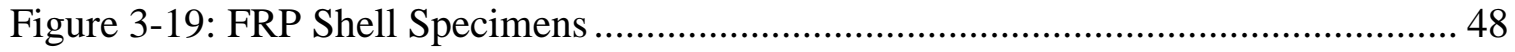

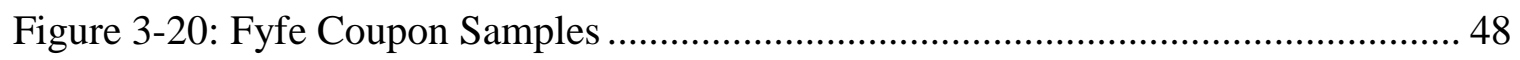

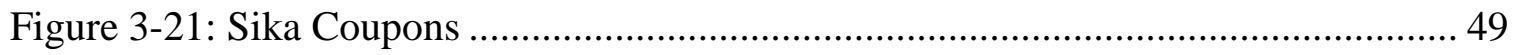

Figure 3-22: Aquawrap® Coupon Samples......................................................... 49

Figure 3-23: Phenolic Coupon Samples ............................................................... 49

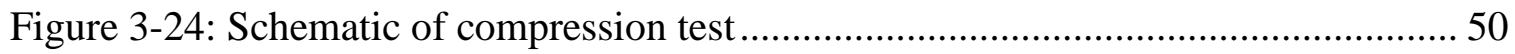

Figure 3-25: Example of compressive loading ..................................................... 51

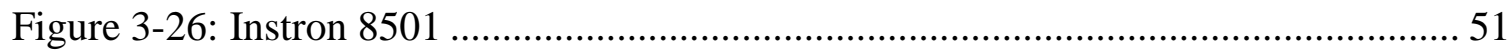

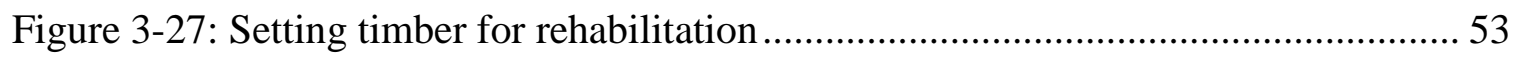

Figure 3-28: Trimming insulation for simulated rehabilitation ................................. 53

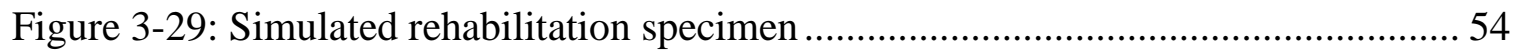

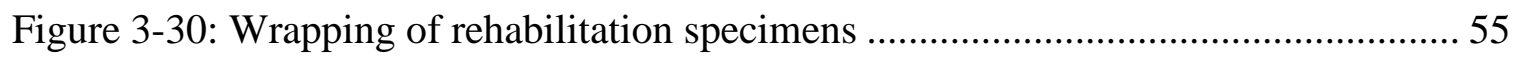

Figure 3-31: Schematic of Simulated Rehabilitation Test ........................................ 56

Figure 3-32: Simulated rehabilitation evaluation testing setup ................................ 56

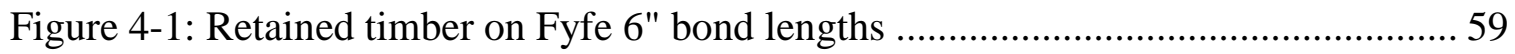

Figure 4-2: Retained timber on Fyfe 12" bond lengths .......................................... 59

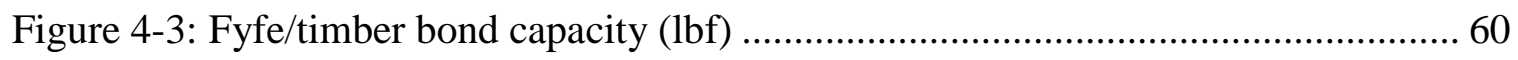

Figure 4-4: Limited retained timber on Aquawrap ${ }^{\circledR}$ 6" and 12" bond specimens ............ 62

Figure 4-5: Retained timber on Aquawrap ${ }^{\circledR} 12$ ” specimen with sliver of timber............. 62

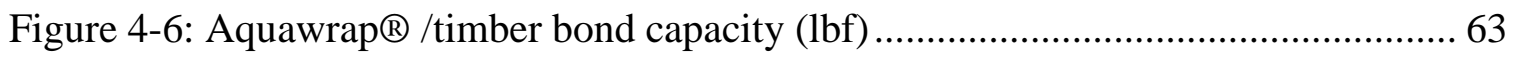




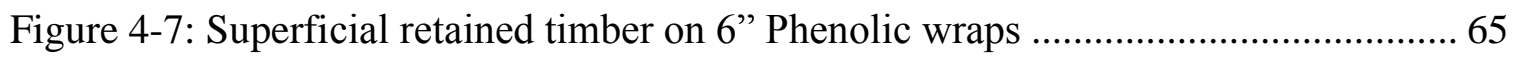

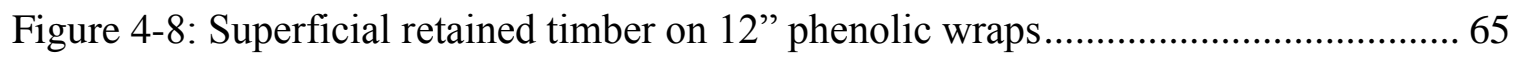

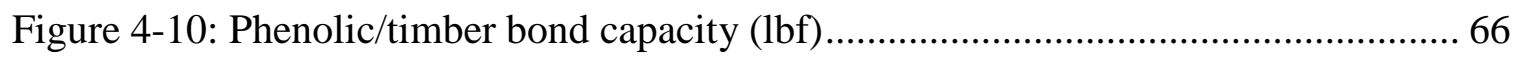

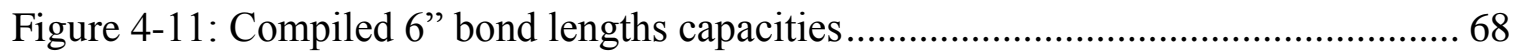

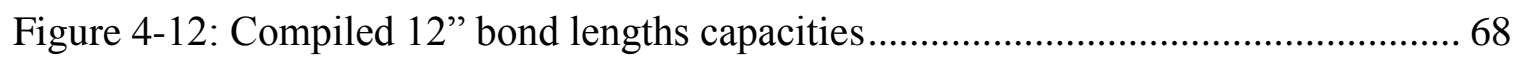

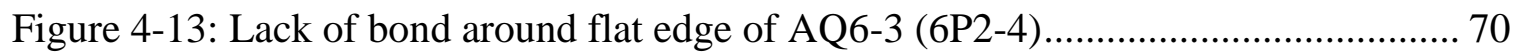

Figure 4-14: De-bond on rounded Aquawrap® bond specimen AQ12-2 (12P2-2) ........ 71

Figure 4-15: Phenolic bond specimen with pad to negate eccentricity $(12 \mathrm{p} 2-1) \ldots \ldots \ldots \ldots . . . . .72$

Figure 4-16: Failure from fiber crushing on AQ 12-1 (12P1-1) and AQ 12-3 (12P3-3).. 73

Figure 4-17: Fyfe Pull-off Carriers (Timber) ..................................................... 76

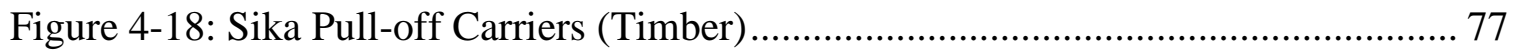

Figure 4-19: Aquawrap ${ }^{\circledR}$ Pull-off Carriers (Bond line) ............................................ 78

Figure 4-20: Phenolic Pull-off Pucks (Timber) ….................................................. 79

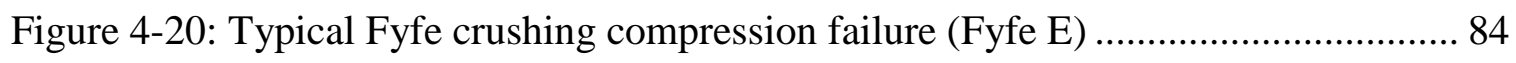

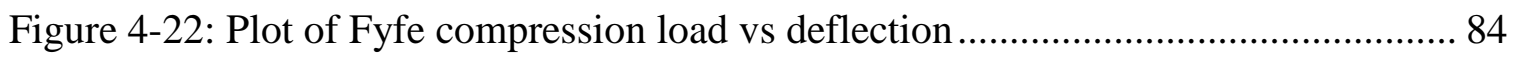

Figure 4-23: Typical compressive failure of sika system (Sika C) in the fibers.............. 86

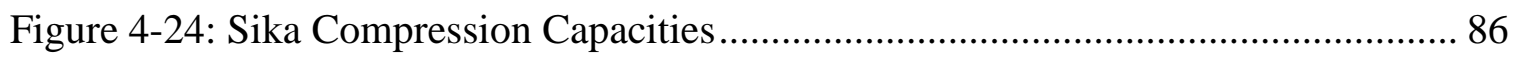

Figure 4-25: Elastic Local Buckling Failure of Aquawrap ${ }^{\circledR}$ (3 layers) .......................... 88

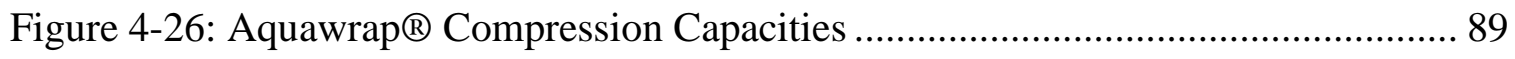

Figure 4-27: Typical compressive failure mode in the phenolic shell (Phenolic A) ....... 90

Figure 4-28: Phenolic Compression Capacities ..................................................... 91

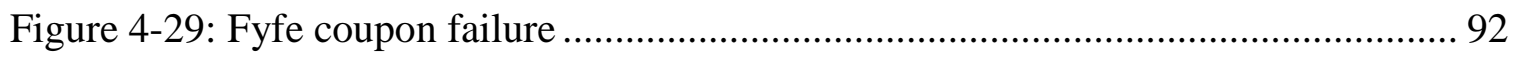

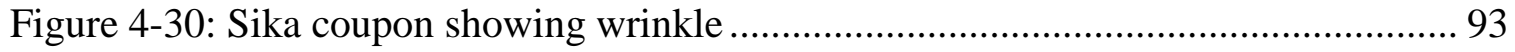


Figure 4-31: Sika Coupon Failure at wrinkle 93

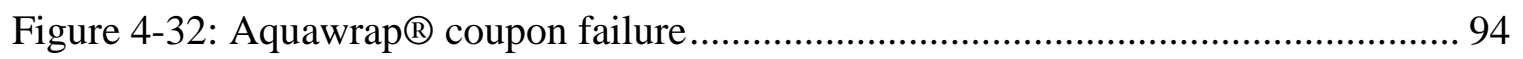

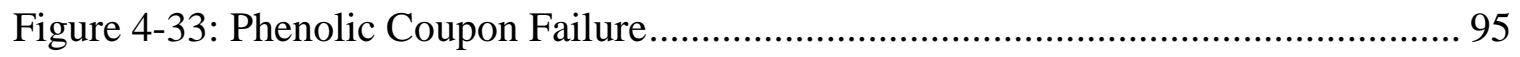

Figure 4-34: Load vs deflection for three layers of wrap ........................................ 98

Figure 4-35: Load vs deflection for five layers of wrap ........................................ 98

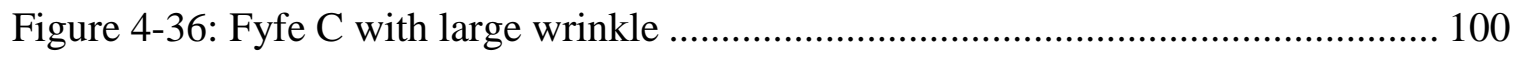

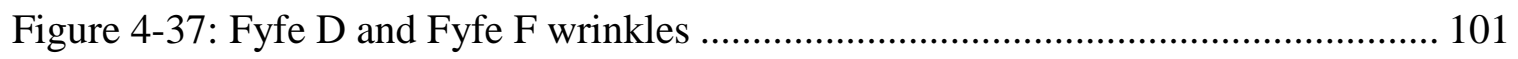

Figure 4-38: Lack of parallel bearing surface (Sika D) ......................................... 102

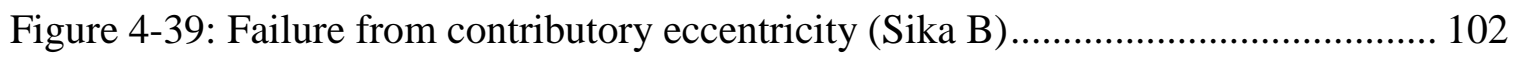

Figure 4-40: Testing head rotating to remove eccentricity ..................................... 102

Figure 4-41: Fyfe A with incorrect placement in Instron (placed too far to the right) ... 103

Figure 4-42: Load versus position behavior of wraps on the piles ............................ 111

Figure 4-43: Fyfe simulated rehab failure .......................................................... 111

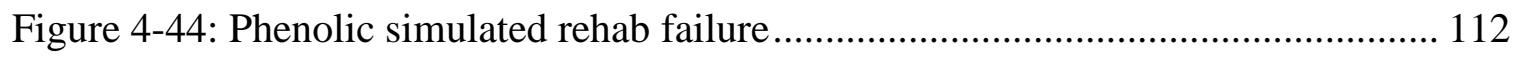

Figure 5-1: Preliminary evaluations of bond length with 4", 8" and 10" bond lengths . 124

Figure 5-2: Preliminary evaluations with 4", 8", and 10" bond lengths....................... 124

Figure 5-3: Comparing perfectly smooth and non-smooth surfaces........................... 125

Figure 5-4: Perfectly round versus non-round results.......................................... 125

Figure 5-5: Preliminary evaluations with stricture wrapping and vacuum bagging ....... 126

Figure 5-6: Vacuum bagging versus stricture wrapping ...................................... 127

Figure 5-7: Crushing reinforcement using timber sections and adhesive .................... 128

Figure 5-8: Crushing reinforcement using PVC and pipe clamps ............................ 129

Figure 5-9: Crushing reinforcement using three additional layers of wraps ................. 129 


\section{LIST OF TABLES}

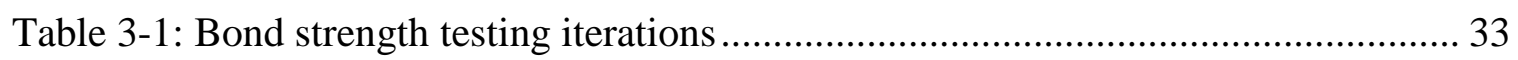

Table 3-2: Fabric dimensions for bond test ...................................................... 36

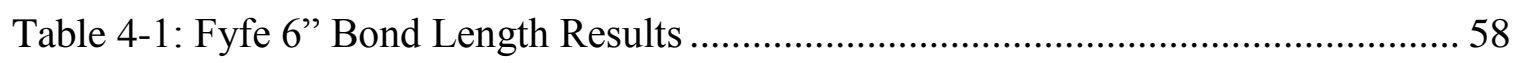

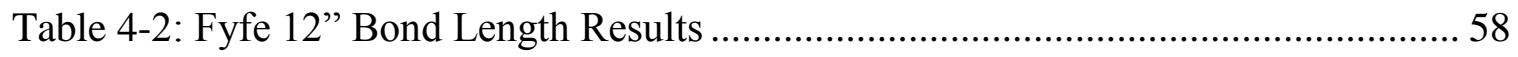

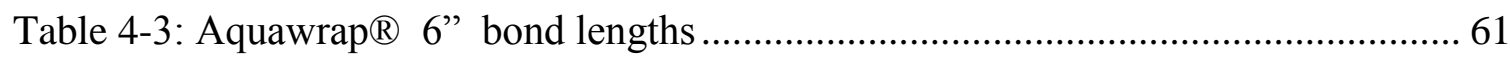

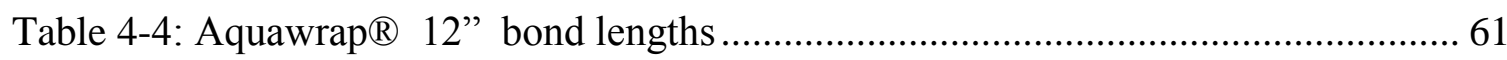

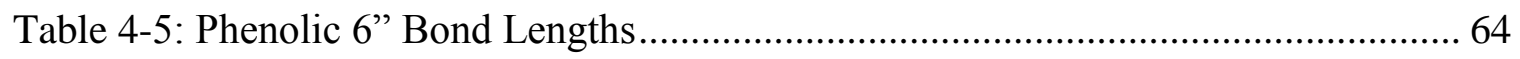

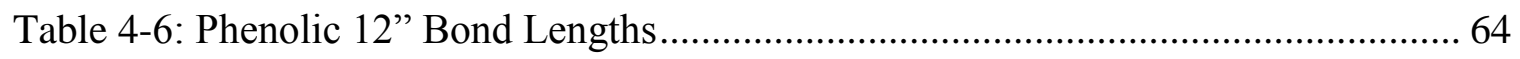

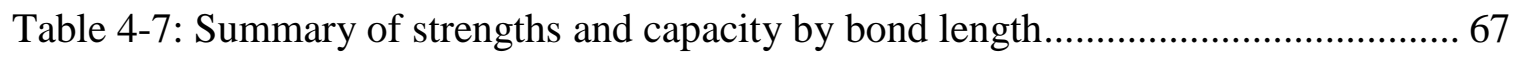

Table 4-8: Factors influencing lowered bond capacity ......................................... 74

Table 4-9: Fyfe Pull-off Bond Capacity and Strengths .......................................... 75

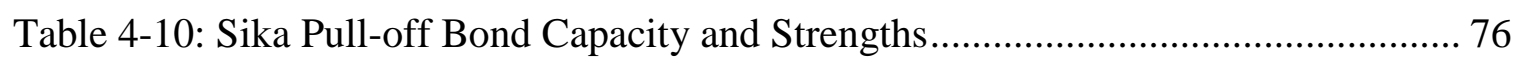

Table 4-11: Aquawrap ${ }^{\circledR}$ Pull-off Bond Capacity and Strengths ................................... 77

Table 4-12: Phenolic Pull-off Bond Capacity and Strengths ..................................... 78

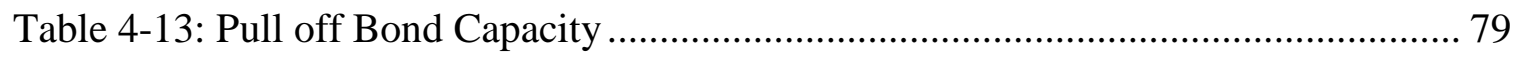

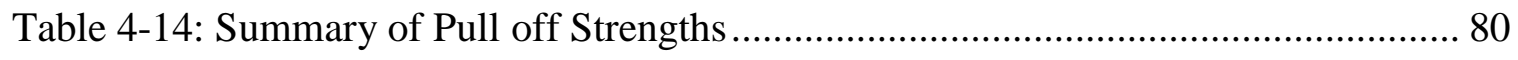

Table 4-15: Comparison with pull off and average pushout bond strengths (psi)........... 81

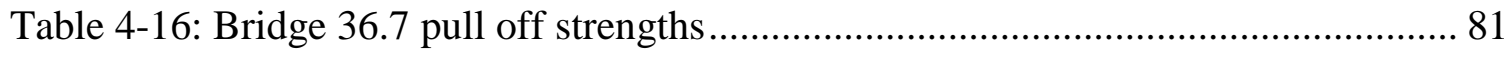

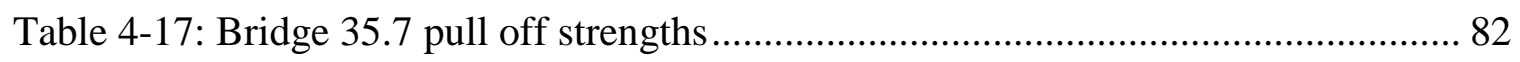

Table 4-18: Fyfe Three Layer Compression Results ................................................ 83

Table 4-19: Fyfe Five Layer Compression Results ................................................ 83

Table 4-20: Sika Three Layer Compression Results ................................................... 85 


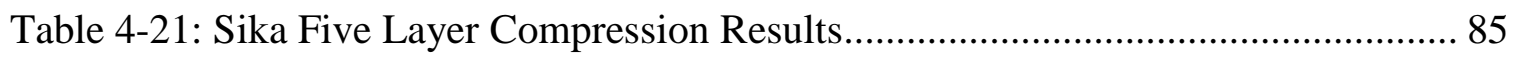

Table 4-22: Aquawrap ${ }^{\circledR}$ Three Layer Compression Results ....................................... 87

Table 4-23: Aquawrap ${ }^{\circledR}$ Five Layer Compression Results ....................................... 87

Table 4-24: Phenolic Three Layer Compression Results ............................................ 89

Table 4-25: Phenolic Five Layer Compression Results............................................. 89

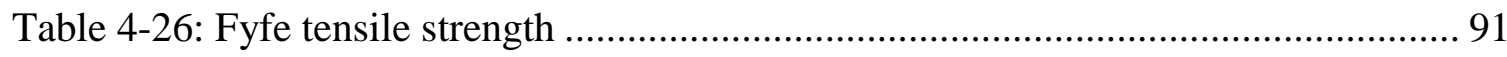

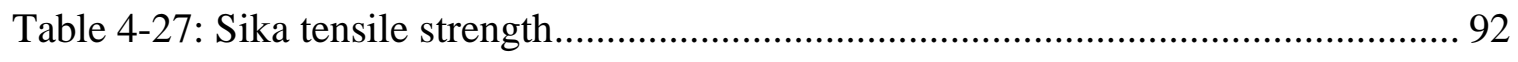

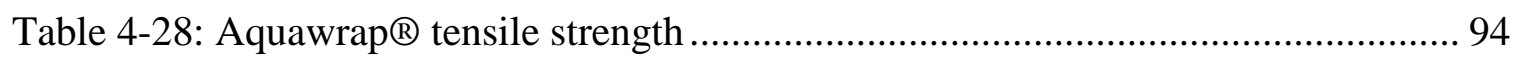

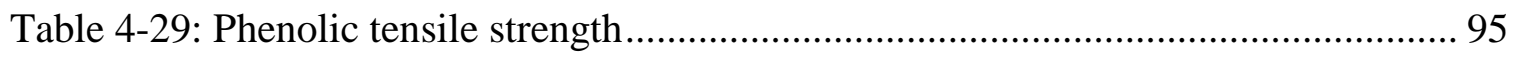

Table 4-30: Summarized Tensile Results ............................................................. 95

Table 4-31: Average compressive capacity by number of wraps (lbf) ............................. 96

Table 4-32: Average compressive strength by number of wraps (psi) ......................... 96

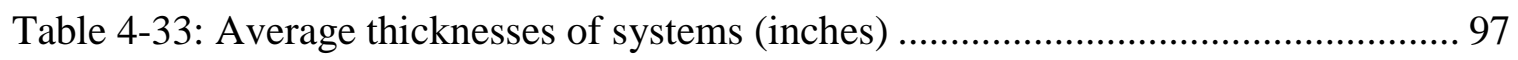

Table 4-34: Summary of influences on compressive capacity .................................. 104

Table 4-35: Compression vs tensile strengths compared (psi) .................................. 104

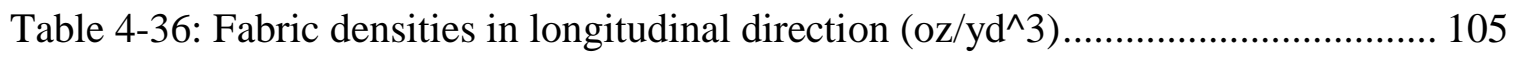

Table 4-37: Comparing compressive strengths and fabric densities versus Fyfe ........... 105

Table 4-38: Comparing tensile strengths and fabric densities versus Fyfe .................. 106

Table 4-39: Average moduli based on compression and tension .............................. 107

Table 4-40: Variations in Tensile Coupon Testing Results ....................................... 108

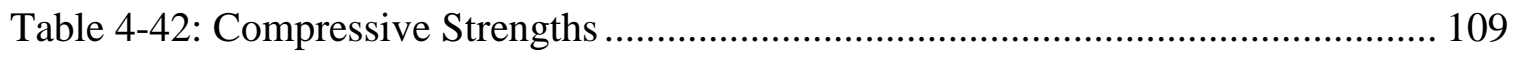

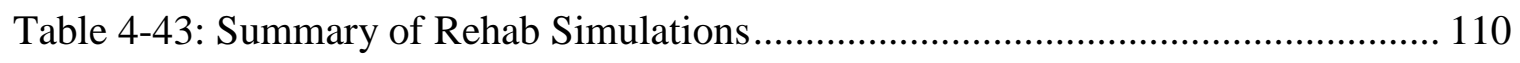

Table 4-44: Comparison with compression strength (psi) ...................................... 112 
Table 4-45: Comparison with bond strength (psi)

Table 5-1: Average compressive strength by number of wraps (psi) ... 116 


\section{CHAPTER 1 INTRODUCTION}

\subsection{Background}

Timber piles, exposed to fluctuating moisture contents and fungi, can often deteriorate past serviceable limits, requiring replacement or repair. In Louisiana, contractors have used Fiber reinforced polymer (FRP) composite wraps to repair decayed timber piles, but bridge engineers coordinating these repairs lack design guidelines and strengthening equations to properly specify required number of wraps and recommended bond lengths. While organizations have successfully used FRP wraps for the repair of timber piles, efforts have generally been focused on confinement strengthening using a variety FRP products on timber piles ranging in condition from "new" to severely decayed (large cross section loss). Due to variability in compressive capacity of decayed timbers, extrapolating number of wraps used in these repairs to generalized strength predictions is not feasible. From a design perspective, it is critical to be able to predict the strength of FRP wrap repairs independent of the decayed pile. Furthermore, none of the past efforts focused on FRP/timber bond development lengths, a crucial design element for contact critical repairs since failure to develop good bond strengths will result bond slippage.

To address these two main issues (compressive capacity independent of timber and develop of required bond lengths), this study will evaluate compressive capacity of FRP wraps with varying wrap number of wraps in the form of hollow tubes and bond strength on new timber piles with varying bond lengths. The hollow tubes allow for accurate strength evaluations without the presence of timber. Evaluating the bond lengths on new timber piles allows for comparative evaluations between systems without the variability of decay. Different FRP systems commonly 
used for timber repairs will be evaluated to provide bridge engineers with accurate design values for a selection of systems.

\subsection{Objectives}

- To review published literature for the behavior of deteriorated timber piles, current repair methods, and determine current FRP wrap systems suitable for timber piles with respect to factors influencing the strength and durability of FRP to timbers adhesion.

- To evaluate various commercial FRP wrap systems for bond and compressive capacity via laboratory tests.

\subsection{Organization of Thesis}

- Chapter 2 contains the literature review

- Chapter 3 contains descriptions of the bond and compression testing of the FRP systems

- Chapter 4 includes the analysis and discussion of the tests conducted for all four systems

- Chapter 5 contains the conclusion along with recommendations for future work

- References 


\section{CHAPTER 2 LITERATURE REVIEW}

\subsection{Introduction}

The literature review was conducted to provide a comprehensive understanding of factors important for the repair of FRP wraps for timber piles. The factors investigated included timber deterioration, preservative treatments, and common repair methods. Studies utilizing FRP wraps on timber piles were investigated to determine FRP wrap systems for the laboratory evaluations.

\subsection{Deterioration Mechanisms of Timber Piles}

According to AASHTO Maintenance Manual for Roadways and Bridges, timber piles typically require limited maintenance unless exposed to open-air environments [1]. However, exposed timber piles are subjected to numerous decay mechanisms, leading to cross section loss and significant structural degradation. This decay is especially pronounced in the splash zone at water lines. As a natural material, timber is vulnerable to deterioration from a variety of sources if not properly maintained and chemically treated. Sources of decay include moisture, fungi, insects, abrasion, heat, holes, corrosion from metal connectors, and chemicals. Of these, the most common factors affecting piles are moisture, fungi, insects, and abrasion.

\subsubsection{Influence of Moisture}

Timber that is protected from the environment and with a relatively low moisture content is very durable, and timber continuously submerged in fresh water will show very limited decay [2]. However continuous exposure to fluctuating moisture contents freeze-thaw cycles splash zones of wet-dry cycles chemical exposure causes the wood to swell and shrink irregularly, 
producing internal damage and external surface checking [3]. Each cycle increases the damage to the wood [4] and exposes more of the wood cells to the water [3]. In addition to the damaged inflicted by the swelling and shrinking, moisture cycling can also leach out preservatives and natural extracts that protect the wood from fungi. Because of this, timbers at the water line are especially vulnerable to decay and degradation [5].

\subsubsection{Influence of Fungi}

Fungi are organisms that consume wood as food. They are made up of interconnected hyphae that spread through pits or penetrate the cell walls of the wood. When elongated, hyphae release enzymes that break down the wood to be absorbed by the fungi as food. When sufficiently fed, fungi will produce fruiting bodies and release spores to infect other nearby wood. Fungi compromise the cell walls of the wood and create section loss and weakening in infected areas. Four main forms of fungal deterioration have been identified: brown rot, white rot, soft rot [5] and heart rot. While slightly different in how they deteriorate the cells and how they spreads through the wood, all of these fungi cause decay and section loss.

\subsubsection{Brown Rot}

Brown rot attack cellulose and hemicellulose. Since cellulose provides much of the strength of the cell wall, wood capacity can be reduced rapidly even if it may appear undamaged visually. Brown rot spreads far from the site of the initial visible damage making it the most dangerous form of rot as it commonly more wide spread than suspected via visual inspection [5].

\subsubsection{White Rot}

White rot fungi in its advanced stages gives the wood a soft texture. Unlike brown rot, white rot attacks the lignin as well as the cellulose and hemicellulose of the wood cell wall causing 
more structural damage. Its enzymes are mostly located near the source hyphae, and it is not noticeable in the early stages [5].

\subsubsection{Soft Rot}

Soft rot fungi generally attack the outer wood shell of moisture exposed wood, but they are not considered as great of a threat as brown and white rot [5].

\subsubsection{Heart Rot}

For larger diameter piles, a form of fungal decay called heart rot can be extremely damaging. During treatment, only the first few inches of the piles can be penetrated by the preservative leaving the interior heartwood to be unprotected. As the timbers dry out, the piles shrink causing splits and checks on the surface. Through these splits, fungal spores can enter the inner heartwood and decay the unprotected core. Though the outer material may remain intact, the interior becomes completely hollowed out over time as the fungi consume and digest the timber pile [6].

\subsubsection{Influence of Insects}

Insects such as termites, beetles, and marine borers can also be encountered in different environments. Because they consume the wood as a food source, they cause pile section loss and softening. Furthermore their burrowing creates openings in the wood through which fungi can enter [7]. 


\subsubsection{Influence of Abrasion}

In stream beds, the erosion of the base of piles is a commonly encountered problem in many applications. Such deterioration occurs from the impact of materials floating in the water and weathering from the flow of the stream [7].

\subsection{Preservative Treatments of Timber Piles}

While not all causes of deterioration can be prevented, treating the timbers with chemical preservatives will decrease the likelihood of deterioration occurring. Preservatives are chemicals that are toxic to fungi and insects, which are coated or injected into the timber. Two broad categories of preservatives have been implemented, oil based and water based. While some of these preservatives may no longer be commonly used for new construction, it is important to be familiar with them as the type of preservative used to treat the timber pile can influence repair and rehabilitation decisions of existing structures.

\subsubsection{Oil Based Preservatives}

Although numerous oil based preservatives are available, perhaps the most common is creosote. Creosote based preservatives were widely used historically due to their long history of high performance in every environment, except when exposed to marine borers. It protects the wood from weathering and limits moisture content changes, preventing checking and splitting. Unfortunately, creosote is harmful to marine environments and has recently been restricted in those applications, and creosote leaching makes surface preparation and cleaning difficult. Pentachlorophenol and copper naphthenate are also commonly used oil based preservatives [8]. 


\subsubsection{Water Based Preservatives}

Water based preservatives are more expensive but leave a clear surface finish that can be stained or painted. Of these preservatives, Chromated Copper Arsenate (CCA) commonly is utilized in marine and brackish environments to protect against marine borers and mitigate the environmental impact of oil based preservatives. However it contains heavy metals and can be hazardous to human health causing its restriction in residential areas. Additional water based preservatives include copper naphthenate and ammoniacal copper zinc arsenate [8].

\subsection{Rehabilitation Techniques for Timber Piles}

While chemical treatments are typically effective in preserving the treated areas initially, they often can leach out of the wood over time leaving the timber piles to deteriorate. Once deterioration has occurred, remedial action is required to restore the piles to their original structural capacity. After a review of the literature, the following methods were determined to be commonly used by state DOTs for structural repair: concrete jacketing, posting/splicing, supplemental pile placement, fiber reinforced polymer (FRP) composite shells, and fiber reinforced polymer (FRP) composite wraps.

\subsubsection{Concrete Jacketing (Concrete Confinement)}

Concrete confinement can be utilized for severely deteriorated piles with section loss of $10-50 \%$ [9]. The concrete protects the pile from further abrasion and weathering, and provides an increase in compressive strength. To begin, the surface of the pile in the area to be repaired is cleaned and a steel reinforcement cage is placed around the pile with spacers to ensure proper alignment as seen in Figure 2-1. A flexible form consisting of either a fiberglass jacket or a corrugated metal pipe is placed around the pile and secured at the base. After the bottom of the 
form is sealed, concrete is pumped into the top of the form. After placement, the top of the concrete is sloped to allow water to run off [10]. In wet environments, cofferdams would need to be constructed around the base of the pile to allow the implementation of the repair method. Material costs were as low as $\$ 20$ per linear foot, but that estimate does not include labor cost [11].

The main disadvantage is if the concrete encasing the timber pile cracks, the timber pile will be exposed to possible deterioration. Additionally the ability to transfer load between the timber and concrete is considered questionable, particularly when considering bearing strength of decayed timber [11]. In confined spaces, placement of the rebar and jacketing can be challenging [10].
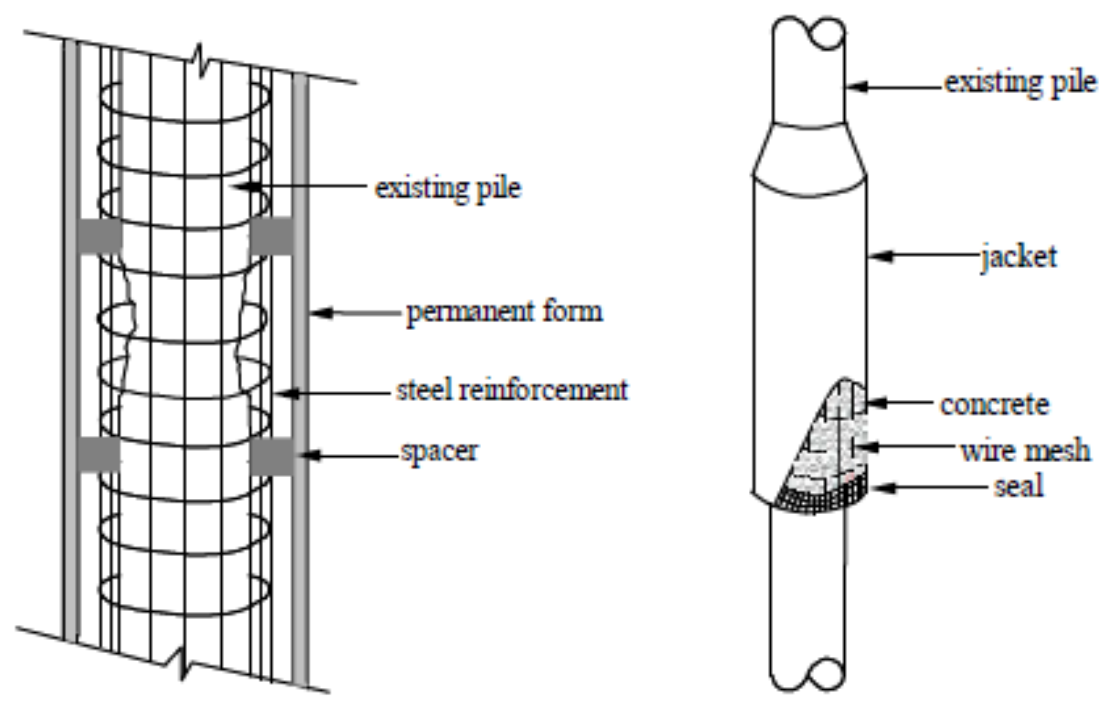

Figure 2-1: Concrete jacketing repair [10]

\subsubsection{Posting/Splicing}

Posting/Splicing is utilized for repair of deteriorated piles at or above ground level. It is very useful for badly deteriorated piles with extreme section losses because it completely removes the deteriorated section and replaces it with a new pile. "Posted" piles can still transfer axial compression forces, but the pile remains weak in flexure [12]. AASHTO recommends that timbers 
on the end bents not be repaired with splicing because the overturning moment on the back wall could cause the splice to fail [1]. To complete a post repair as shown in Figure 2-2, the area around the pile is excavated and a strut is installed using a hydraulic jack to support the pile cap. The damaged pile is removed below the permanent moisture line. A new treated post is installed in the place of the removed pile and secured [10]. The post section can be secured using a wide range of methods including concrete jacketing, timber fishplates, drift pins, steel side supports, epoxy injection, and FRP wraps [13]. Costs can vary for each post between \$126- \$252 depending on the material used for the splicing not including labor costs [11]. Service life will vary depending on the materials used to secure the posting.

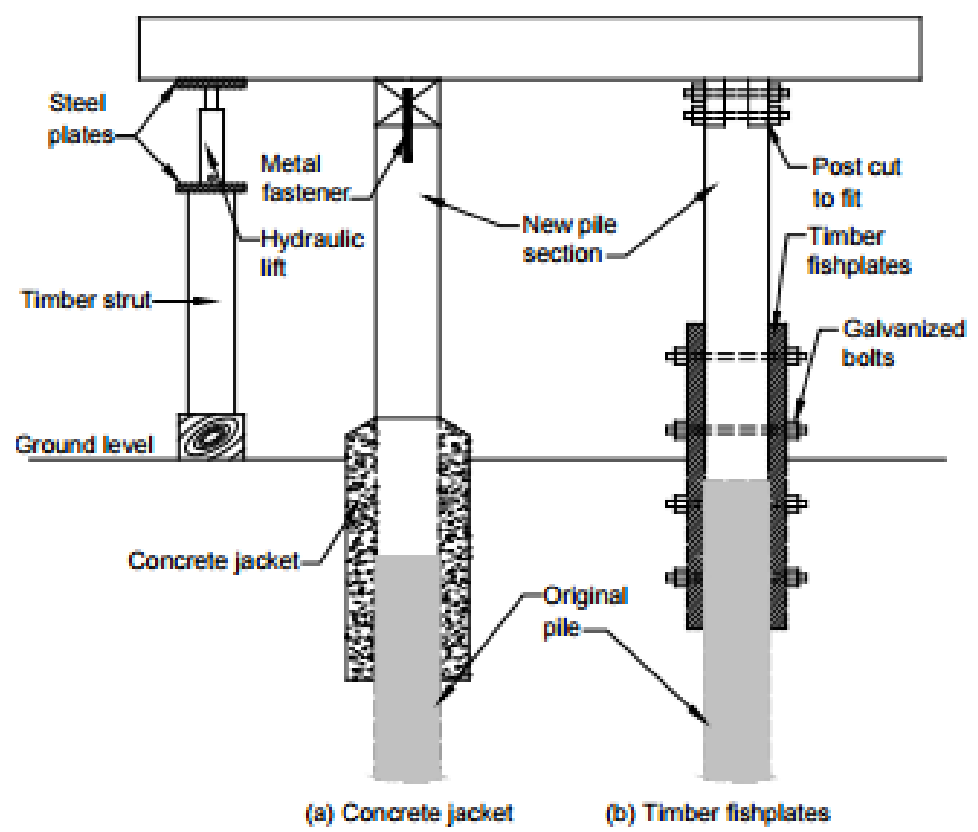

Figure 2-2: Posting/Splicing Repair of Timber [9]

Bridge traffic must be rerouted during repair, and cutting out the damaged section and jacking up the bridge requires extensive amounts of equipment and a solid surface for jacking. Installing a new post is also very difficult for bridges with limited clearance. Furthermore, 
discontinuous load distribution can take place throughout the pile due to the difference in cross section or variations in the timber between the new post and the original pile.

\subsubsection{Supplemental Piles}

In cases where the section losses are too great for other repair methods, supplemental pile placement or replacement is sometimes the only solution. The supplemental piles are generally timber or steel columns [1], [13]. As seen in Figure 2-3, an opening in the deck is cut to allow for the placement of the supplemental pile and the pile is driven into the ground. Once embedded, it is laterally pulled to alignment under pile cap and shimmed. For timber piles, a drift pin is installed and for steel piles, expansion bolts are utilized [13]. The repair method is very expensive and requires bridge closure; therefore it should only be considered as a last resort.

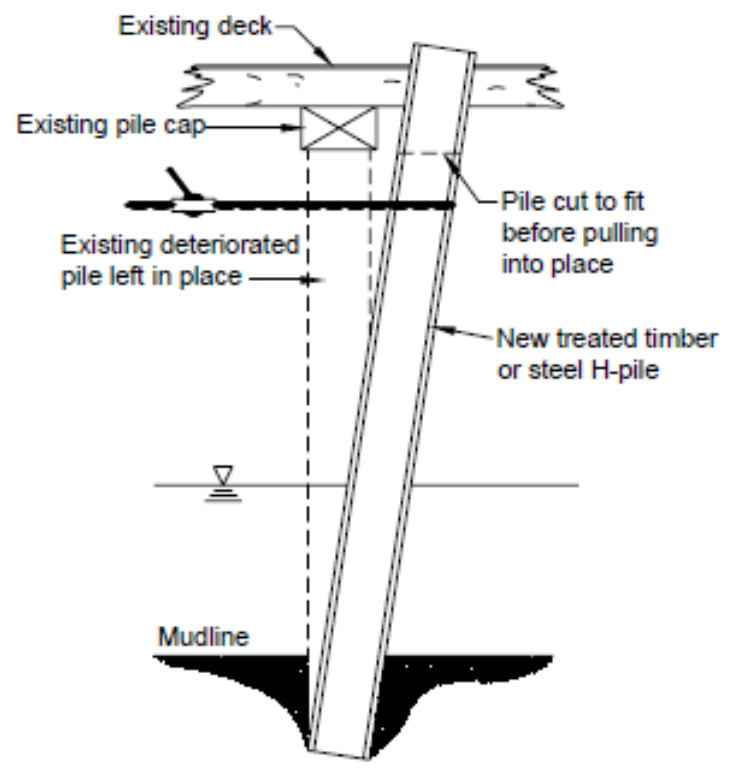

Figure 2-3: Supplemental pile [9]

\subsubsection{Prefabricated FRP Composites Shells with Grout}

FRP composite shells with grout are used in situations that require both structural strengthening and protection from further biological decay. The primary example would be a 
deteriorated marine wharf pile infested with marine borers. The damaged wood around the pile is removed and the remaining timber treated to ensure the decay does not continue after the repair. The FRP shell is placed around the pile and sealed at the base, but an opening remains at the top of the pile. Utilizing this opening, grout (cementious/epoxy with aggregate) is pumped into the shell as seen in Figure 6. Once cured, the rehabilitation process is complete [13].
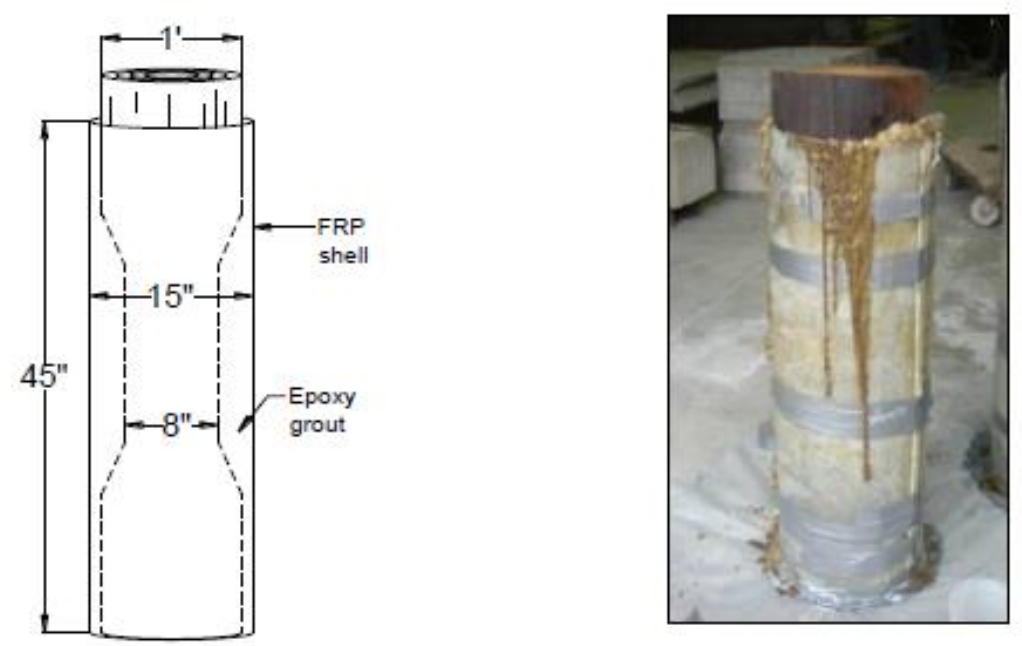

Figure 2-4: Prefabricated FRP shells [6]

The repair time is relatively quick and typically does not interfere with the daily traffic of the road system. Furthermore the shell protects the pile from further environmental exposure. The repair method is more expensive than other methods, one source citing $\$ 600$ for linear foot [11]. Additionally due to the higher stiffness provided by the grout, stress concentration can develop above and below the repaired portion of the pile due to the differential stiffness between the two materials [14]. This can result in premature bearing failure.

\subsubsection{FRP Composite Wrap Systems}

FRP composite wraps are utilized in situations that require protection as well as strengthening. Typical practice in marine environments is to remove damaged and decayed portions of the pile and thoroughly clean the pile to remove all remaining fungi. If substantial 
section losses exist, a filler material (either a cementious grout or a resin mixed with wood particles or expanding wood filler) is placed into the void and secured until cured. The surface is then primed with a coupling agent to improve bonding. After this, the pile is wrapped and smoothed to ensure no voids are present. Typical wrap layers vary from $2-5$ wraps [13]. The studies were not clear on methods used to apply even pressure. Once cured, the wraps protect the piles and increase their capacity under live loads. Good strengths, efficient labor utilization, limited traffic disruption, load transfer optimization, and costs make this repair method desirable [15]. One source listed costs as low as $\$ 10$ up to $\$ 50$ per square foot [11]. The source did not clarify whether it indicated square foot of wrap or square foot of pile.

\subsubsection{FRP wraps with Splicing}

If the timber is deteriorated to the point that fillers cannot be used, a hybrid repair technique can be used combining splicing and FRP wraps. As seen in Figure 2-5, the decayed section is cut out and a new pile section is inserted, then FRP wrap is used to secure the splice in place [12]. This method has only been evaluated in the lab and has currently not been field tested to the authors' knowledge.

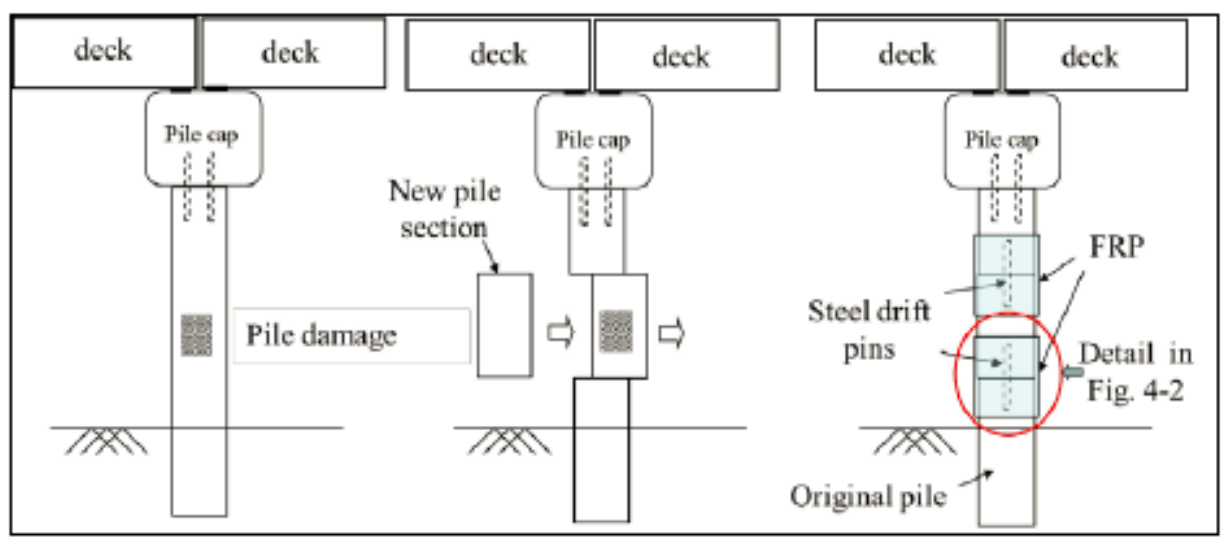

Figure 2-5: Hybrid FRP wrapping method [12] 


\subsubsection{Summary of Rehabilitation Techniques}

Methods for repairing piles include concrete jacketing, posting/splicing, supplemental piles, prefabricated FRP shells, and FRP wet-layup composite wraps. Compared to the other repair methods, FRP wraps are less intrusive, provide strengthening as well as protection from biological decay, and can be more cost effective than other methods. Additionally, placing the wraps is far less labor intensive than moving a very heavy section of wood and replacing it with another section. For these reasons, FRP wraps are good choice for rehabilitation of deteriorated timber piles.

For the purpose of this study, case studies of FRP wraps on timber are presented that provide information needed for design. Evaluations were included for number of wraps and types of FRP systems commonly utilized.

\subsection{Studies of FRP Composite Wraps on Timber Piles}

\subsubsection{Influence of number of wraps}

Najm et al evaluated forty poplar timber samples in compression with carbon fiber reinforcement in an inorganic matrix [16]. All samples were short columns to avoid buckling. The carbon fiber reinforcement utilized included unidirectional fabrics and continuous strands wrapped in spirals with varying spacing. Unidirectional sheets were tested in one and three layers each. Evaluating the different configurations revealed that the columns with full confinement from the unidirectional sheet performed well, but those with three fabrics showed even higher performance levels [16]. Carbon fibers were selected for this study to provide a direct comparison with research that utilized carbon wraps and an organic matrix (as opposed to an inorganic matrix). 
Hagos utilized unidirectional glass FRP wraps for the rehabilitation of damaged columns using grout as filler material. The configuration used is shown in Figure 2-6. For his testing, he assumed that the grout carried the majority of the load. Based on this assumption, he calculated the number of wraps to be used based on concrete confinement theory. While not directly applicable because of the grout, he determined that two wraps provided more strength than one [14].
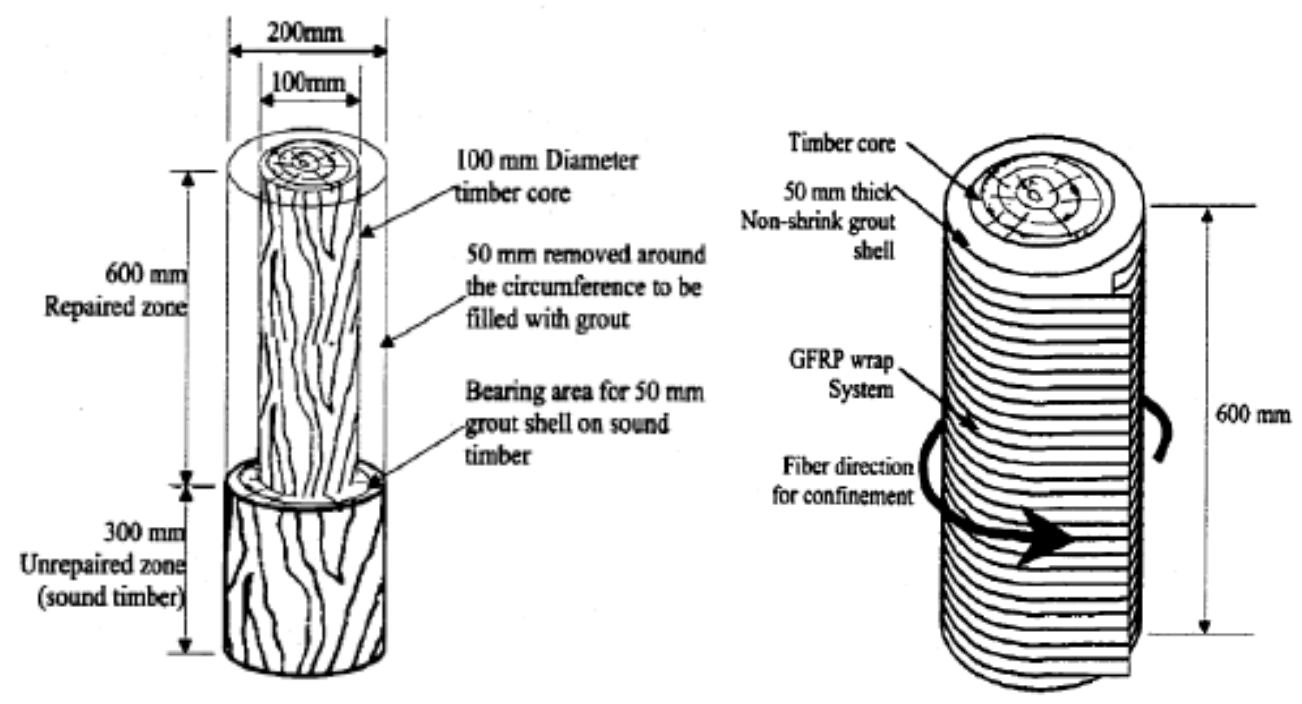

Figure 2-6: Hagos wrapping scheme [14]

Illinois Transportation Center used Fyfe FRP wraps to enhance the capacity of piles repaired with posted sections. Members were evaluated in compression and compression-flexure. The number of wraps required to obtain strengths greater than the unretrofitted capacity was determined by trial and error. The number of wraps used varied from 9-10 [12]. It should be noted that the axial capacity was already enhanced by the new pile section. Therefore, the wraps were simply strengthening an already adequate member.

From these studies it can be seen that more wraps increase compression capacity, but it is not known if this trend continues linearly with more wraps. 


\subsubsection{Common systems utilized for service conditions}

\subsubsection{Fyfe Wrap System:}

In a study in Nova Scotia at Halifax Cable Wharf, marine timber piles experiencing severe deterioration from freeze/thaw cycling and marine borers were rehabilitated using glass FRP wraps and prefabricated shells [17]. The wet layup consisted of Tyfo® SHE-51 with saturated with Tyfoß SW-1S, an underwater epoxy and were used primarily just for protection from future decay. It was found that the wet layup wraps from this system increase shear capacity and provide confinement [17].

\subsubsection{Sika System:}

Sika shells were used to repair piers on a NYC Passenger Ship Terminal [18]. The shells were placed around the deteriorated pile after which grout was pumped into the shell to complete the repair. While shells were used for this particular evaluation, Sika also provided a line of wrap products similar to the Fyfe system.

\subsubsection{Aquawrap ${ }^{\circledR}$ by Air Logistics Corporation}

Aquawrap ${ }^{\circledR}$ were selected for a repair of timber piles on a bridge by an unnamed transportation department. Several of the piles on the bridge had deteriorated to the point of separation between the top and bottom portion of the piles. In this study, a BP-4 primer/coating was applied to fill cracks in voids. After curing for two hours, Aquawrap ${ }^{\circledR}$ was then applied, covering all decayed areas above and below the water line. After another two hour cure, epoxy was injected to restore the pile to a full cross sectional area [19]. 


\subsubsection{WVU-CFC phenolic systems}

WVU-CFC has successfully utilized phenolic wraps on timber piles several times in the past on the South Branch Valley Railroad (SBVR) in Moorefield, West Virginia. The first applications were completed during 2002 on three bridges using 28 oz/sq. yd bidirectional GFRP (glass fiber reinforced polymer) fabric with phenolic resin [20] [21]. The repairs followed the following process: First the decayed portion of the pile was removed and then the surface was sanded. Sharp edges was rounded down with an edger to reduce stress concentrations. Shrink wrap was applied around the pile and then filler material consisting of saw dust mixed with the phenolic resin was inserted into the make shift mold then allowed to cure [20]. For piles with large section losses, a plastic mold was applied and then the filler was applied [21]. After this the piles were primed with phenolic resin and cured for 24 hours. Wraps were saturated with phenolic formaldehyde resin, wrung out to remove excess resin, and applied with two layers. Field testing using locomotives before and after load application revealed improved strain distributions [21].

In 2010, glass fiber reinforced polymer composite using the same systems were use on eleven timber bridges for a total of 57 piles. The wraps used were $12 \mathrm{oz} / \mathrm{sq}$. yd bidirectional Eglass fabrics with Cascophen RF G1149 resin. Decayed portions of timber were removed, and the surface was sanded before being coated with the saturating resin as a primer. Filler material consisting of the saturating resin mixed with sawdust was used to fill voids in the wood. For wrapping, two layers of wraps were applied and secured with shrink wrap for 24 hours [22]. 


\subsection{FRP Wrap Design for Timber Pile Repair}

\subsubsection{Basics Theory of FRP Wrap Design}

Fiber reinforced polymer composites systems are composed primarily of two elements: 1) fabric made up of fibers that provides the bulk of the strength and 2) resin system that binds the fibers together and ensures transfer of forces. For this study, manufacturers selected provide the optimum combinations of fabric and resin. Factors to be considered are influences on wrap installation and filler materials.

\subsubsection{Influence of Wrap Installation}

Prepreg is the pre-impregnation of a fiber matrix with a partially cured resin. It allows for more control over the final fiber distribution and eliminates the need for time consuming fabric cutting and saturating. Because it is a prefabricated material, it is more expensive than wet-layup. As the resin is already partially cured, the materials has a limited shelf life. Although it has a higher volume fraction and a more controlled resin content than wet layups, the higher costs and limited shelf life make it less attractive for field use [23].

Wet lay-up or hand lay-up is the simplest and most widely used FRP manufacturing method. Fabrics are soaked in resin and applied to the timber and rolled flat using a hand tool. For proper application, the fabric has to be carefully wrung after being soaked in resin. Less desirable elements include fumes, labor utilization, non-uniform resin distribution, and longer curing rates. Wet layup is the least expensive FRP wrapping method [23].

After the wraps are install, some form of external pressure is applied. In the past, WVUCFC utilized shrink wrap plastic wrapped tightly applied to circumference [20], [22]. Systems 
have also been developed called stricture wrap that can be used to apply uniform pressure during the curing process similar to shrink wrap.

\subsubsection{Published Design Codes for FRP wraps for Timber Piles}

\subsubsection{Design Codes for FRP Wraps}

Currently, no design codes have been established for the wrapping of timber members. In light of this, design philosophies can be modified from ACI 440.2R-08, FRP wrapping of concrete substrates [24]. ACI 440.2R-08 was developed for general use and provide useful requirements on the storage, handling, installation, inspection, and maintenance of FRP wraps. AASHTO also has developed a code FRP repair of concrete members, "Guide Specifications for Design of Bonded FRP Systems for Repair and Strengthening of Concrete Bridge Elements" [25]. The manual, in addition to design guidelines for FRP systems, also includes surface preparation and inspection information [25]. The design information is not currently directly applicable to timber structures, but some of the material will be useful for developing design guidelines for timber FRP wraps.

\subsubsection{Design Codes for Timber Piles}

The AASHTO LRFD Bridge Design specifications specify that the nominal axial compression resistance for timber piles is based upon the strength of the pile in compression parallel to the grain, calculated as per Section 8.8.2 [26]. This calculation includes a reduction based on the Euler buckling strength of the pile, which is specified in 10.7.3.13.4 to include the depth under the soil to fixity, but is otherwise based on the adjusted compression strength parallel to the grain multiplied by the area of the pile [26]. The allowable compression strength can be found in Section 8.4.1.4 which lists the same values found in the American Wood Council's 
National Design Specification (NDS). AASHTO specifies various adjustment factors based on the size, condition and time effects. AASHTO specifies that timber piles must conform to the AASHTO M168, which references ASTM D25 for timber piles [26].

Further design information can be found in the following:

- ASTM D25 provides general pile specifications for new timber piles, including straightness, cutting and peeling requirements and minimum butt and tip sizes for different lengths and classes of piles [27]

- ASTM D2899 discusses how to establish the allowable stresses in round timber piles $[28]$

- The Timber Pile Design and Construction Manual combines information from the NDS and ASTM standards into one complete guide for the design of new timber piles [29]

All of the above sources are developed on the basis that the pile is sufficiently braced. If not properly braced, a pile must be designed according to AASHTO 8.10.2 for loads subjected to both flexure and compression. This equation combines the moment and axial forces, including using the Euler buckling strength [26]. The information from these various codes and standards will be modified as needed to incorporate simple design calculations for determining the enhancement needed by the FRP wraps. 


\subsection{FRP/Timber Bonding}

\subsubsection{Preparation Factors Influencing Bond}

\subsubsection{Influence of Surface Preparation}

Before the bonding, free or loose material should be removed from the surface of the wood so adhesion will be directly with the main wood surface. After the removal of loose material, the pores of the wood surface should be opened to allow the adhesive to enter increase interlock. Methods commonly used in factories settings include sawing, sanding, or cutting by knife [30]. While cutting with a knife produces the most desirable finish and leaves open pores without residue, sanding would be most reasonable preparation method for field applications. Like planeing with a knife, sanding opens up the pores of the wood, increasing wettability. Well-sanded surfaces are flat, allowing an even spread for the adhesive which improves the bond. Despite these benefits, sanding too intensely can abrade portions of the wood substructure weakening the surface. Furthermore, sanding can leave a layer of dust in the pores that inhibits bonding [30]. Because of this, it is recommended that the surface should be lightly sanded with high grit sand paper. Once a surface is sanded, the adhesive should be applied immediately to prevent the collection of contaminates on the surface [31]. For field installation, it would seem that the most imperative issue would be creating a smooth surface and removing loose material. Therefore, despite its drawbacks, sanding would still be a reasonable surface preparation method.

\subsubsection{Influence of Moisture Content}

Changes in moisture content negatively affect the bond between FRP and wood due to the hygrothermal stresses developed by the swelling of the wood [32]. For factory bonding, it is recommended that the wood be conditioned to the same or higher design moisture content (say 
$50 \%$ ) that a wood pile will be subjected to in the field [33]. This is done to prevent the development of hygrothermal stresses as the wood swells. Elements that were preconditioned showed better durability than those that were dry and later exposed to moisture [34]. However, excessive moisture prevents bonding because wood pores are filled with water preventing the adhesive from entering. If the surface is over dried, extracts can diffuse to the surface of the wood which can interfere with bonding [31]. For field applications, it would be more important to have a dry surface to create a strong bond than to be concerned about moisture cycling as this will occur at the water line or splash zone.. It would be more reasonable to select a moisture compatible resin. For many resin types, the surfaces should be dry which requires diverting the water and drying the wood.

\subsubsection{Influence of Applied Pressure}

Proper application of pressure is important because it forces out entrapped air, brings the adhesive into contact with wood, squeezes the adhesive into a uniform film, and holds the FRP in place during curing. For curing applications as would be utilized in field work (minimum 70 degrees), it is suggested to apply pressure for at least 24 hours [22]. Failing to apply uniform pressure results in weaker than expected bond between the wood and FRP [35]. Manufacturers have specific pressure ranges for their adhesives, which should be followed to the best of the contractors ability during field installation of FRP wraps.

\subsubsection{Primers/Coupling Agents Influence on Bond with Treated Timber}

\subsubsection{Primer Types}

Primers are important for the improved bonding of plastics to both treated and untreated wood [31]. Common coupling agents found in the literature are hydroxymethylated resorcinol (HMR), resorcinol formaldehyde (RF), and polyurethane (PU). Hydroxyl methylated resorcinol 
(HMR) was developed in the 1990s to improve bonding of epoxies, vinyl esters, and phenol resorcinol formaldehydes with CCA treated and untreated wood [36], [37]. Resorcinol formaldehyde is a common wood adhesive used to bond wood laminates. Polyurethane, common resin used to manufacture composites, has been shown to produce good bonds between polymers and wood [31].

For the purposes of this study, it is sufficient to note that generally primers provide improved bond strengths on different types of treated timbers. For this study however, the saturating resin will be applied and not any additional special primer. Since no durability analysis will be included in this portion study, no in depth analysis of the various types of primers and their influences on treated timbers is included.

\subsubsection{Bond Evaluation Techniques}

\subsubsection{Bond via Pushout Test}

Bond evaluation methods utilized in this study utilized "pushout" test methods pioneered by Lopez- Anido [38]. In that particular study, FRP shell systems were evaluated by grouting the shells around timbers in the configuration shown in Figure 2-7. The wood was offset from the shell which allowed for timber and shell to be loaded independently causing the force to be transferred through the bond. The method worked well as the thickness of the shell prevented fiber crushing at the base. 


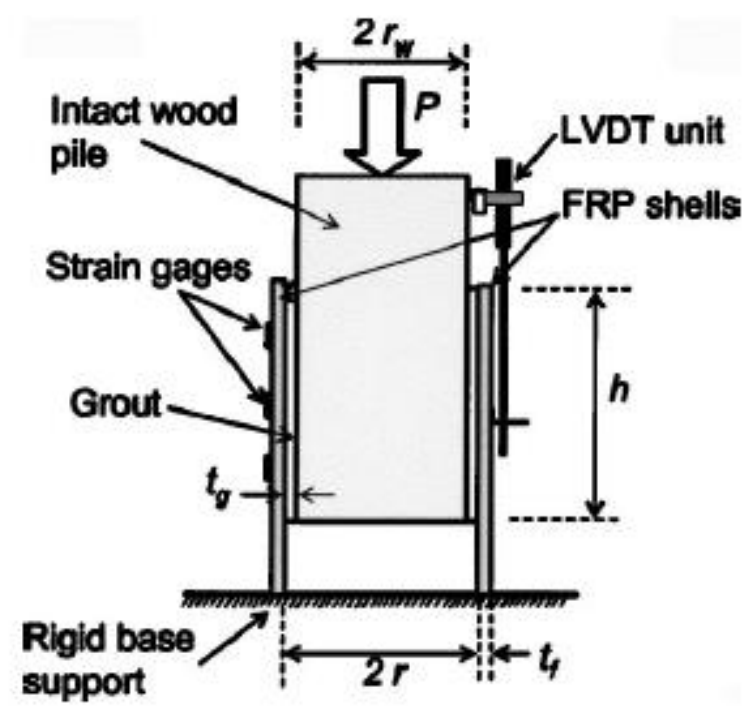

Figure 2-7: Testing schematic of apparatus used by Lopez-Anido [38]

\subsubsection{Bond Strength via Pull off Test}

For pull off evaluations, ASTM D7522 has been successfully utilized to evaluate the bonding strength of FRP wraps to concrete piles. This test method uses 2-inch diameter carrier that is bonded to the FRP surface on a previously cut core hole to create a distinct test area and then pulled off using a calibrated tester [39]. By modifying this method for timber with FRP wraps, valuable data relating pull off strength with bond shear strength can be gathered.

\subsection{Summary}

From the literature review, the following observations were determined to be further evaluated:

- Timber deterioration mechanisms were identified.

- FRP wraps are viable alternative to some of the other timber repair methods available. 
- Influences impacting bond strengths were identified for this study and taken into account for bonding of the bond systems. These included moisture, surface preparation, primers, and pressure during application.

- Fyfe, Sika, Aquawrap ${ }^{\circledR}$, and Phenolic systems have all been utilized successfully in the past to improve timber bonding therefore they will also be evaluated in this study. 


\section{CHAPTER 3 TESTING OF FRP WRAP SYSTEMS}

\subsection{Introduction}

\subsubsection{Limitations in current research}

Current studies of FRP wraps have been focused primarily on determining, on a case by case basis, the number of wraps required to restore specific deteriorated timber piles to adequate capacity through confinement strengthening. Furthermore, the studies have evaluated a variety of FRP products on several different types of timber piles, whose diameters and decay rates vary widely from study to study. While such data is useful for those specific applications, the wide variations that exists among studies makes extrapolating the results into general guidelines difficult.

An alternative approach would therefore be to first determine the potential failure modes of FRP wraps on timber piles and then to evaluate specific FRP products, used in previous studies, through a battery of tests to determine ultimate strengths of these failure modes. Such an approach would allow not only eliminate the current issues related to variability but would also provide useful data for developing simplified strength predicting equations. To begin this process, failure modes were first identified.

\subsubsection{Failure modes of FRP wraps on timber piles}

For FRP wrap repairs, four failure modes are possible: 1) compression failure in the timber, 2) loss of composite action between the FRP and the timber (e.i. bond slippage), 3) local compressive failure of the FRP wrap, and 4) global buckling of the FRP wrap. 


\subsubsection{Timber compressive failure}

In the field, degradation between timber piles can vary widely depending on the life of the pile and the environmental conditions in specific locations. Due to this variability of degradation, it is challenging to accurately predict the capacity of decayed timber from a design point of view. To eliminate this large uncertainty, it is therefore most effective to assume that decayed portions of timber in zones of repair contribute zero compressive capacity. Any additional capacity provided will simply be in addition to that accounted for in the design. Compressive failure in timber outside the repair area would have been previously accounted for in the design of the timber pile and will not be considered.

\subsubsection{Loss of composite action between wrap and timber (bond slippage)}

For FRP wraps on columns, two types of strengthening mechanisms can be utilized: "contact critical" or "bond critical". For concrete columns, FRP wraps are often used for "contact critical" application meaning the wraps provide passive confinement and does not fully engage until the concrete is dilated or cracking [24]. Alternatively, "bond critical" systems provide bending, shear, and axial enhancements from the start of external loadings.

For the purposes of design, FRP wraps on timber piles will be assumed to operate as bond critical systems since the behavior the decayed timber under a contact critical application would be difficult to accurately predict. For bond critical systems, failure occurs when the timber and wrap separate from each other (bond slippage) resulting in the loss of composite action between the timber and the wrap. Such a slippage negates the additional strength provided by the wrap returning the pile to its original strength and behavior. The wrap however may still provide some small measure of confinement enhancement, but should enhancement could be considered 
insignificant when compared to the capacity reduction after loss of the bond critical state. In light of this, timber/FRP wrap slippage will be considered a critical area for evaluation in this study.

Much information is available on coupon size specimens related to FRP/timber bonding, but information on global FRP/timber bond slippage is limited. Therefore, to accurately determine the bond slippage strengths, full scale bond evaluations will be conducted on new, creosote timber piles (to eliminate the irregularity present in decayed timber and to account for influences from preservatives). Ultimate bond slippage strengths will be determined for different bond development lengths to allow designers to comfortably specify bond lengths for field installations.

Field methods in the form of tensile pull off testing exist for determining if wraps for bond critical applications are installed properly on concrete substrates. For quality control purposes, it would also be reasonable to use a similar method to establish baseline pull off strengths values to correlate with field installed values in the field.

\subsubsection{Compressive failure of wrap}

In addition to bond slippage, FRP wraps can also fail in compression through compressive crushing based on simple load over area (P/A) compressive strength, which increases based on the thickness of the composite (number of layers). Should such a failure occur, the wrapped section would immediately lose any strength capacity provided by the wrap. The lack of advanced warning from this type of failure make its accurate prediction even more critical.

While compressive design strengths for FRP products are provided by manufacturers, they are based off of small scale specimens (i.e. less than 1 inch wide) evaluated by ASTM standards and these small coupon specimens do not necessarily predict the compressive behavior of full scale repairs. Therefore full scale samples in the form of hollow tubes manufactured with different numbers of wrap will be evaluated in axial compression until failure. By coupling compressive 
strength with wrap thickness (varying layers of wrap) and testing hollow tubes (neglecting timber capacity), the results will provide designers with accurate strength values per number of wraps for future design predictions.

\subsubsection{Buckling of wrap}

For global Euler buckling equation to be valid, the repaired FRP section must span a significant distance given the cross section of a pile, i.e. roughly 10 feet without bracing for a typical 12" diameter hollow composite. For local buckling to control, the thickness of the wrap would have to be so thin that it would fail in crushing before buckling. Thus, the crushing capacity governs the compressive strength of the FRP repair. Buckling will be not considered as failure mode for design purposes.

\subsubsection{Scope}

The objective of this study is therefore to evaluate the ultimate bond slippage strength between wraps and new treated timber piles per a given bond length and to determine the ultimate compressive capacity of wraps with different number of layers. These results will allow for the future creation of simplified design codes for specifying bond lengths and number of wraps for any given loading. Four different manufacturers of FRP wrap systems were selected for these evaluations: Fyfe, Sika, Aquawrap®, and WVU-CFC's phenolic wraps.

\subsubsection{Organization of Chapter}

This chapter is divided into four general sections: materials, bond testing, compression testing, and field rehabilitation simulation.

All materials utilized in the study are presented in Section 3.2. Detailed descriptions are provided for the four types of FRP wraps, and the timber used in testing. FRP/Timber bond tests 
are discussed in Sections 3.3 and 3.4. Section 3.3 presents the test methodology used for the evaluations of two different bond lengths under axial push-out testing, and Section 3.4 describes the evaluations of bond strength under pull-off conditions (ASTM D7522). Compression testing methods are presented for axial compression in Section 3.5. Test methodology for the simultaneous evaluation of bond strength and compression capacities of each system are given in Section 3.6 through the simulated timber rehabilitation tests.

\subsection{Materials}

\subsubsection{FRP Wrap Systems}

For this study, FRP systems using glass fibers were selected as these are relatively less expensive than other fabrics such as carbon or Kevlar and have a similar elastic modulus to timber, which prevents stress concentrations. Furthermore, these systems have been previously used to rehab timber piles as noted in the literature review.

\subsubsection{Fyfe's Tyfo FiberWrap}

The Fyfe system is a wet lay-up unidirectional fabric consisting of Tyfo SEH-51A (27 $\left.\mathrm{oz} / \mathrm{yd}^{\wedge} 2\right)$ with primary fibers with yellow secondary fibers to maintain fiber direction and additional reinforcement. The resin utilized was Tyfo S Epoxy, low viscosity and long workability. It was used previously in past in rehabilitation projects on pier piles (see 2.5.2.1).

\subsubsection{Sika Wrap}

The Sika system is a wet layup system consisting of unidirectional fabric, Sika Hex100G (27 oz/yd^2), and epoxy resin, Sikadur Hex 300. The resin is low viscosity and has a long working time. 


\subsubsection{Aquawrap ${ }^{\circledR}$}

The Aquawrap ${ }^{\circledR}$ system is a pre impregnated polyurethane system with $22 \mathrm{oz} / \mathrm{yd}^{\wedge} 2$ bidirectional fabric. Developed originally for pipe line repair, the system has been expanded to rehabilitation of concrete and timber structures. WVU-CFC has used this system in the past for concrete column repairs and the manufacturer stated it had been used for timber rehab projects [19].

\subsubsection{Phenolic Wrap}

This phenolic formaldehyde wrap system consists of vector ply fabric $\left(18 \mathrm{oz} / \mathrm{yd}^{\wedge} 2\right)$ and Cascophen resin. The system was used by WVU-CFC previously on the repair of railroad bridges due to phenolic's compatibility with timber.

\subsubsection{Timber Specimens}

Timber specimens were used in bond evaluations, pull off testing, and full scale simulated rehabilitation. Testing was conducted on new timber piles to provide a uniform result. New timbers were selected due the variability present in deteriorated piles from the field that would influence the test results. Timber piles were provided from Stella Jones in eight foot lengths and treated with creosote before shipping. Pile diameters varied from 10" to 12 ". A chain saw was used to cut the piles into the required portions for each tests as shown in Figure 3-1. While effective at cutting the pile into appropriate lengths, the hand held saw made it difficult to create straight cuts consistently. Therefore, on many of the timber specimens, surfaces are often non-parallel which created small amounts of eccentricities during testing. Any influences from these eccentricities will be pointed out in the test discussions. 


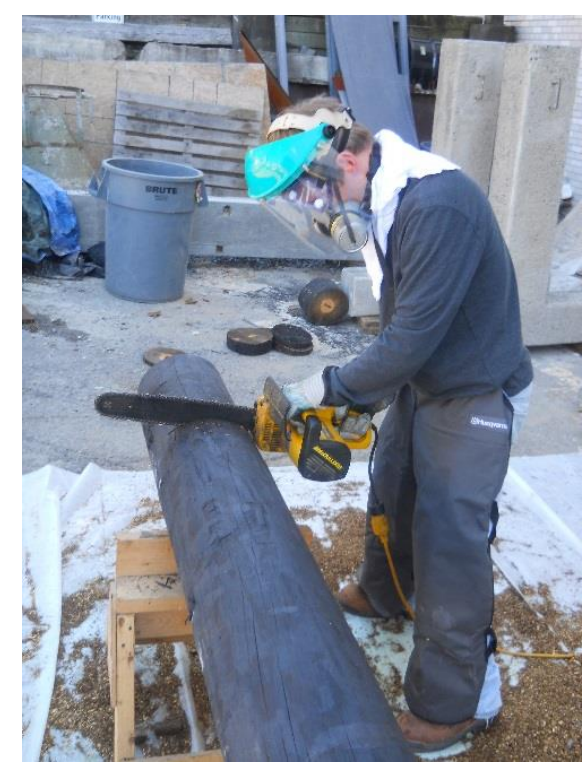

Figure 3-1: Cutting timber specimens

\subsection{Bond Testing (Push Out)}

\subsubsection{Bond Test Development}

The bond test concept is to apply a load to the timber and support the sample only with the FRP system, thus forcing the FRP/timber bond to carry the entire load. Unlike the shell system discussed in 2.7.3.1, the systems to be evaluated in the current study are all wet lay-up composites. Due to the flexible nature of the fabrics during curing, it is difficult to create a uniform base without some form of mold that has the same shape as non-uniform timber pile. This issue is compounded by the fact that the mold must provide for a gap that does not resist any load, but is still capable of holding firm during wrapping. Finally, direct bearing of the composite on the loading surface could result in fiber crushing in the composite at the expected service bond load levels. While crushing of fibers is an issue because of the bearing on the steel plate, in the field no such drastic change in stress will occur as the pile will be a continuous member. Therefore, the fiber crushing encountered is simply a test related issue. 
To solve these issues, several iterations were attempted on 5" diameter copper azole treated timbers to determine the most effective method of creating the specimens. Results from these evaluations can be seen in Appendix A.

After several test iterations, it was found that 6 " and 12" bond lengths were realistic representations for short and long bond lengths. To prevent direct bearing failure or the FRP, a small piece of timber was used to provide a load transfer mechanism from the wrap to the loading platen at the bottom of the sample to prevent crushing of the fabric. A bond breaker was applied to create a controlled bond area accurately while accounting for the variability of hand lay-up. See Figure 3-2 below for a visual representation of the components described above.

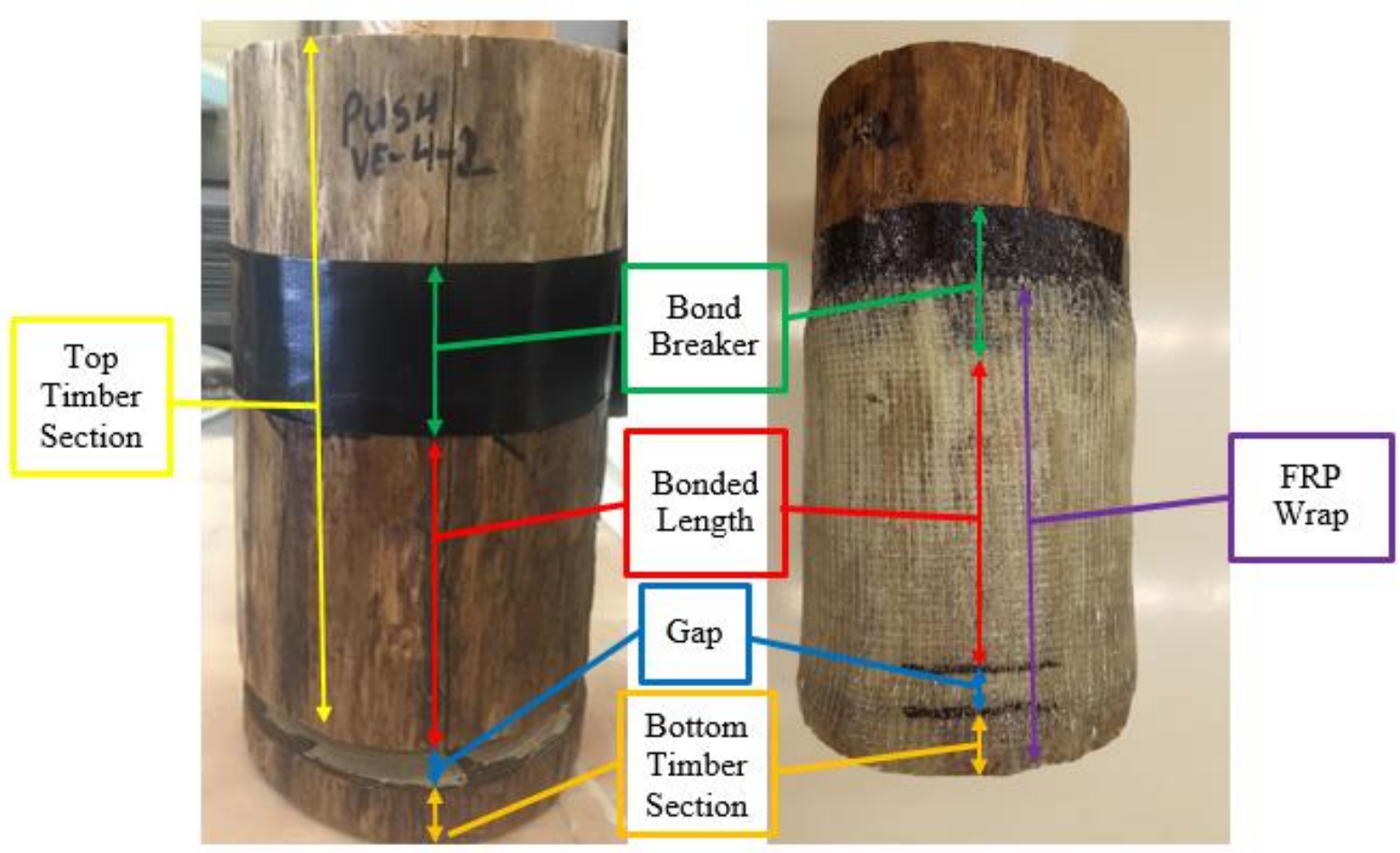

Figure 3-2: Testing schematic of pushout testing portions.

To create a gap between the timber sections, insulation was placed between the lower and upper portions of timber stubs to prevent the fabric from slipping into the gap and keep pooling resin away from filling the gap. It was further determined that additional layers of reinforcement 
around the base of the specimens prevented the composite from buckling in the gap due to local discontinuities or crushing on the load platen. A gap of $3 / 4$ inch was found to be adequate to allow a slippage but not cause buckling in the gap.

From a design perspective, the primary concern was determining the capacity per length of bond to be used in a design. Therefore no strain behavior was evaluated.

\subsubsection{Specimen Preparation}

In order to evaluate average bond strength of FRP composite wraps on timber, timber piles were wrapped with the FRP systems with 6" and 12" bond lengths. Three of each bond lengths were done for each commercial system (Table 3-1). Unidirectional fabric systems were wrapped with three layers to create a [0/90/0] laminate configuration with 0 indicating the longitudinal direction. Bidirectional fabric systems were wrapped with three layers with the fibers being aligned in the longitudinal and hoop directions. Additional layers were applied around the base to provide additional buckling and bearing support.

Table 3-1: Bond strength testing iterations

\begin{tabular}{|l|r|r|}
\hline FRP System & \multicolumn{1}{|c|}{$\begin{array}{c}\text { Short } \\
\text { (6" Bond) }\end{array}$} & $\begin{array}{c}\text { Long } \\
\text { (12" Bond })\end{array}$ \\
\hline Fyfe & 3 & 3 \\
\hline Sika & 3 & 3 \\
\hline Aqua Wrap & 3 & 3 \\
\hline Phenolic System & 3 & 3 \\
\hline
\end{tabular}

For the 6 inch bond length specimens, timber was cut at 10" with a 2" base portion. For the 12 inch bond length specimens, timber was cut at 16" with a 2" thick bottom. After cutting, the bond specimens were assembled with the insulation sandwiched between the top (bond section) and bottom (2") timber portions then screwed together with 3" screws. Insulation was later 
trimmed to match the circumference of the timber (shown in Figure 3-3) and putty was applied to fill any voids created during the trimming of the insulation.

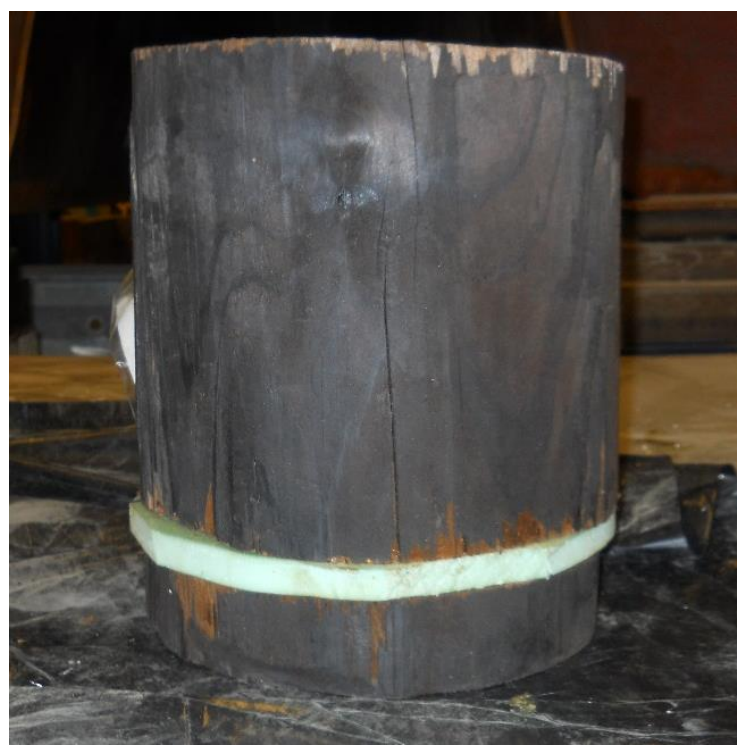

Figure 3-3: Unwrapped timber bond specimen

Samples were then sanded on the surface with an electric palm sander to remove some of the creosote clogging the pores of the timbers to allow for resin penetration, which would improve the bond strength as shown in Figure 3-4. Wax paper was installed at the top of the specimen to provide a bond breaking material that ensured a consistent bond length during wrapping and ensured that the testing would apply load only to the timber. 


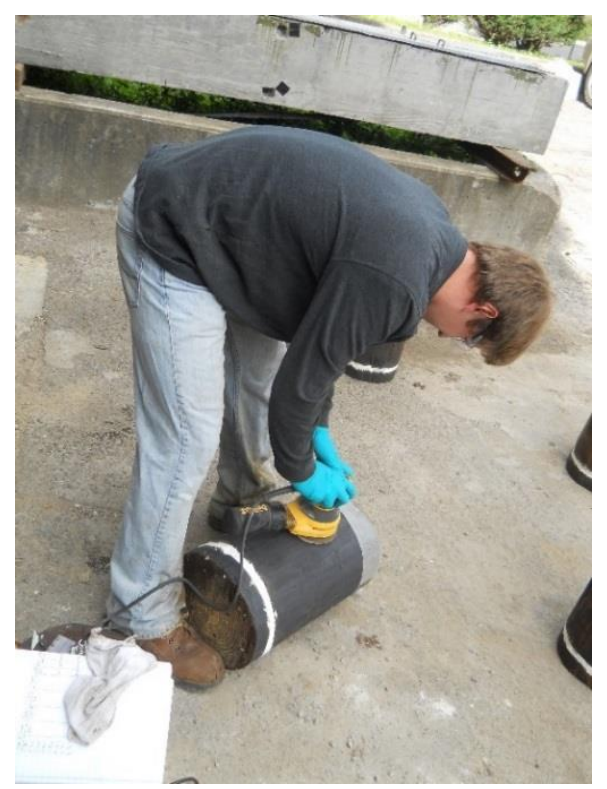

Figure 3-4: Sanding of bond specimens

Samples were wrapped after sanding and the installation of the bond breaker. First, primer consisting of the saturating resin used was applied to the surface of the pile for the Fyfe, Sika, and Phenolic systems (Figure 3-5). Aquawrap ${ }^{\circledR}$ did not provide a primer, but used water to activate the process; therefore the piles were sprayed with a layer of water instead of applying a primer. Next wraps with the dimensions shown in Table 3-2 were saturated by hand with the saturate resin (Figure 3-6) until the surface of the fibers was just beginning to show a coating of resin but not completely submerged with resin. The exception to this process was the Aquawrap ${ }^{\circledR}$ whose wraps were sprayed with water before applying them to the piles (shown in Figure 3-7). Once saturated (activating the prepreg resin) the wraps were wrapped tightly around the timber samples Figure 3-8 and secured in place with stricture wrap (shrink wrap) to provide uniform pressure during curing. The stricture wrap was originally provided for the Aquawrap®, but was also used for the other systems to provide consistent pressure application for each system. The pressure was critical in producing a strong bond between the timber and the wrap. 
Table 3-2: Fabric dimensions for bond test

\begin{tabular}{|l|r|r|}
\hline \multicolumn{1}{|c|}{ Type } & $\begin{array}{c}\text { Height } \\
\text { (inch) }\end{array}$ & $\begin{array}{c}\text { Length } \\
\text { (inch) }\end{array}$ \\
\hline 6" Bond & 10 & 38 \\
\hline 12 " Bond & 16 & 38 \\
\hline
\end{tabular}

Staples were used on the bottom 2" section of pile to provide additional load transfer to the 2" timber base. No staples were used in the upper bond test area as they would provide additional strength that is not realistic in the field as metal staples would rust and fail under field conditions.

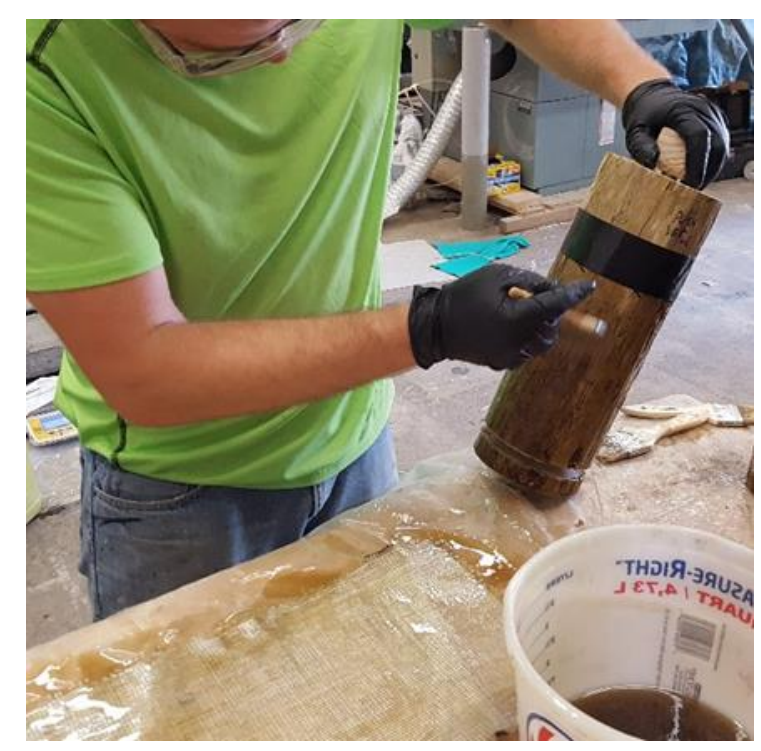

Figure 3-5: Apply primer to smaller mock up sample

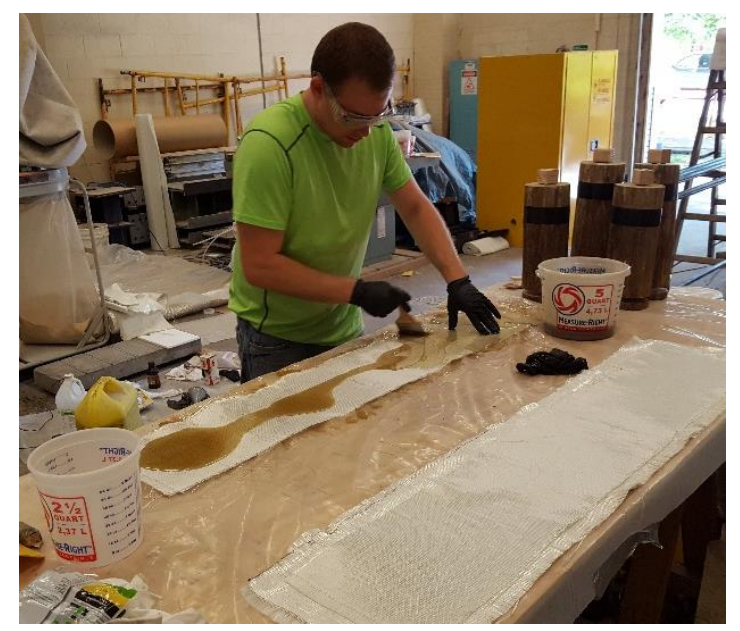

Figure 3-6: Apply resin to wraps for smaller mock up sample 


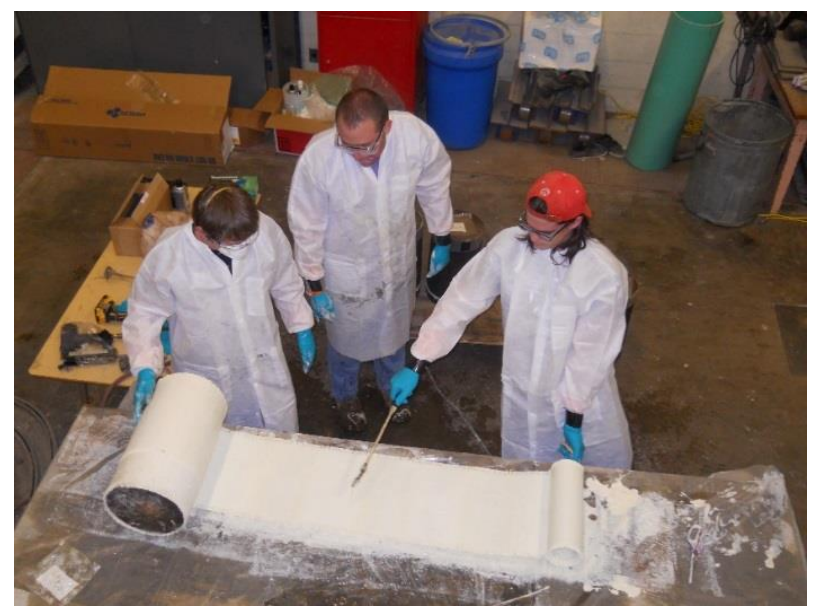

Figure 3-7: Spraying of Aquawrap ${ }^{\circledR}$ for bond testing

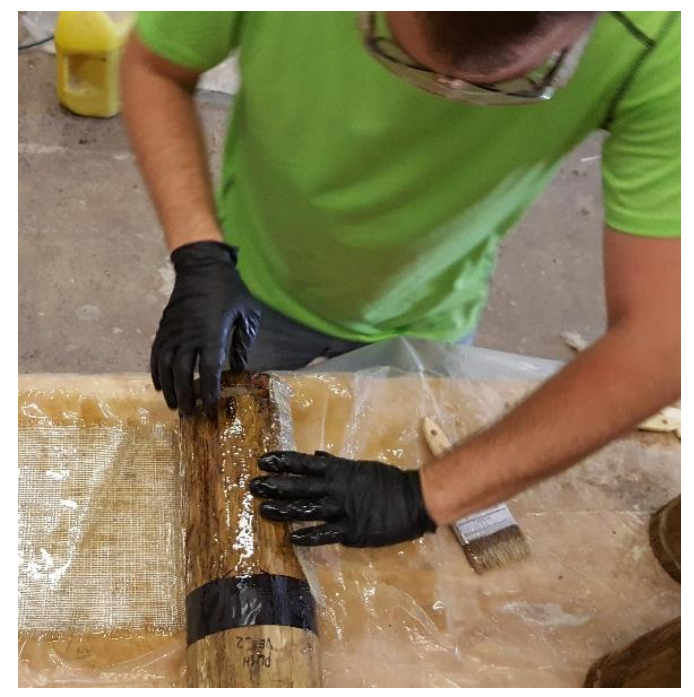

Figure 3-8: Wrapping smaller mock sample

After curing, the screws were removed and the insulation was dissolved with acetone via holes drilled into bottom the base. The insulation and acetone were used exclusively for this particular lab test to create the gap required to allow for bond slippage, and does not represent a procedure that would be utilized in field repairs. The loading surfaces were cleaned of any excess resin and the edges of the composite were trimmed flush with the timber at the base to ensure a uniform loading of both the wrap and the 2 " base. Samples were allowed to cure for at least 7 days before testing. The resins cured within a 24 hours, be 7-day cure was done to allow for full strength 
developments of the bonds. In the field, the bonds upon immediate cure likely will carry any applied loading with increasing strength over time.

\subsubsection{Testing Apparatus}

Specimens were evaluated under compression on WVU-CFC's Instron 1000HDX universal testing machine at a loading rate of 10 kips per minute. To provide a large enough bearing surface for the 10 " to 12 " diameter piles, 2 " thick steel plates (18" x 18 ") were attached to the machines. The configuration for the test is shown in Figure 3-9. A standard bond test is shown in Figure 3-10.

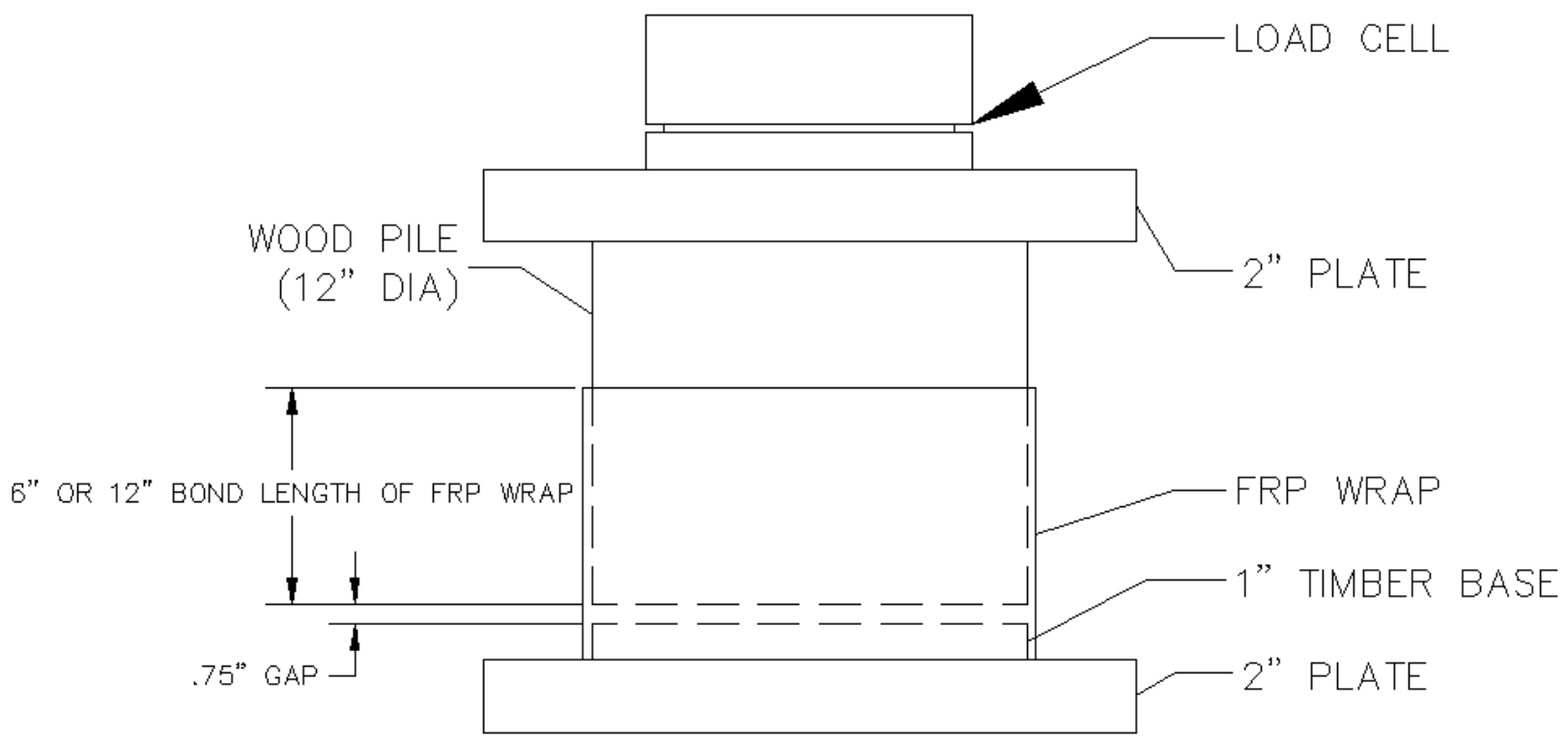

Figure 3-9: Schematic of Pushout Test 


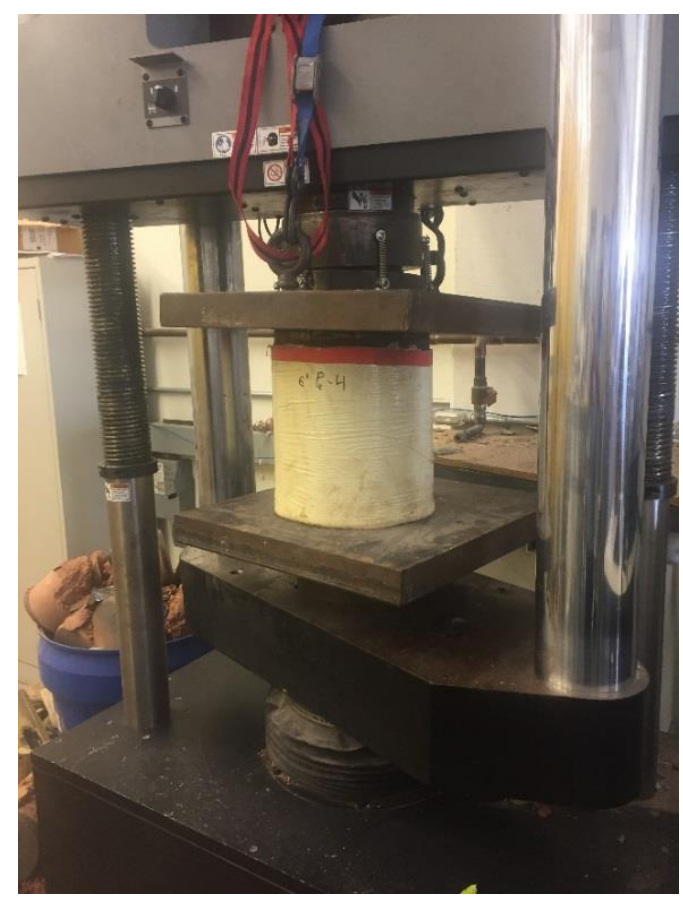

Figure 3-10: Testing configuration for bond test

The samples were loaded at a constant rate until slippage occurred. Slippage was determined based upon evaluating the load versus deflection plots obtained for the results similar to the example shown below in Figure 3-11. The point at which the bond began to slip was identified to be the highest load on the graph. Notice that substantial deflection occurs in the system even after slippage at much lower than maximum loads; this indicates the top portion sliding down into the gap. The small disruption in the load seen around $2000 \mathrm{lbf}$ corresponds a very slight slip of the wrap as it settled under the loading. Evaluating the graph of position versus time (Figure 3-12) clearly shows the point at which the bond slipped as indicated by the rapid increase in deflection (horizontal) with very little increase in time (vertical). 


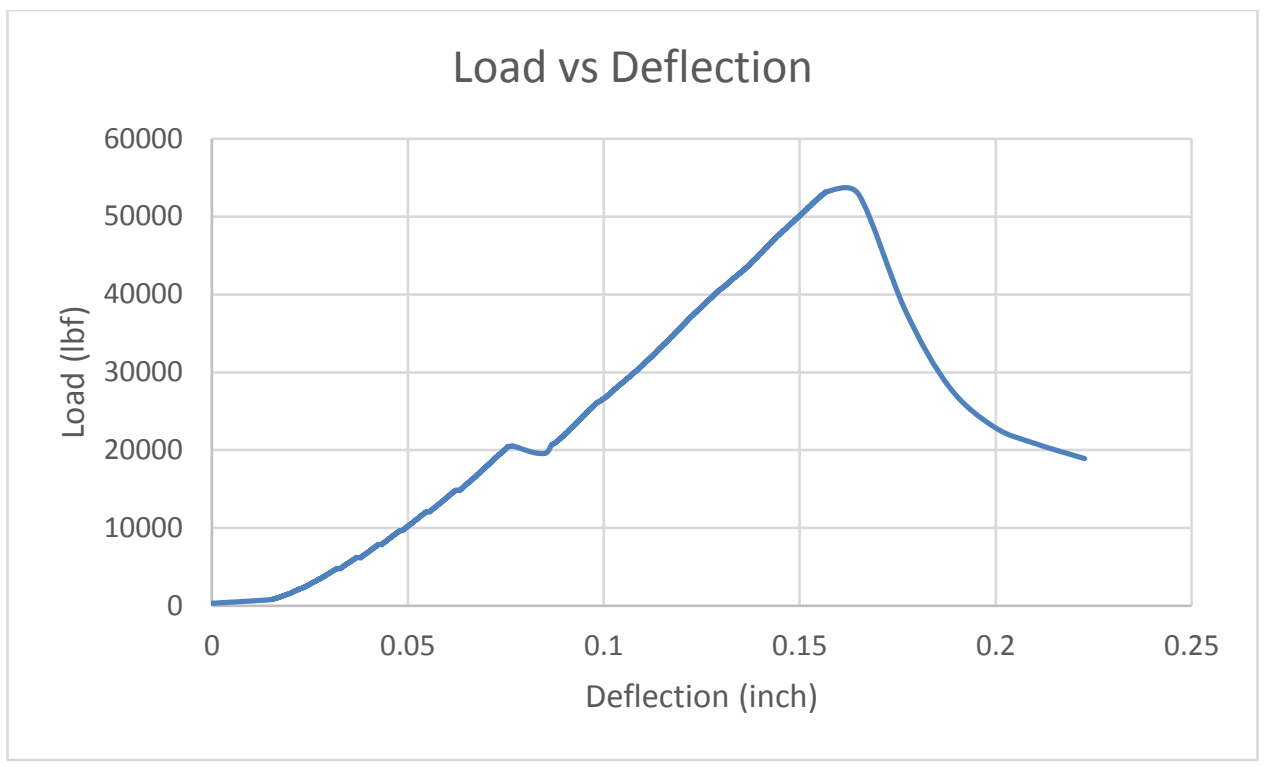

Figure 3-11: Example of pushout load vs deflection plot

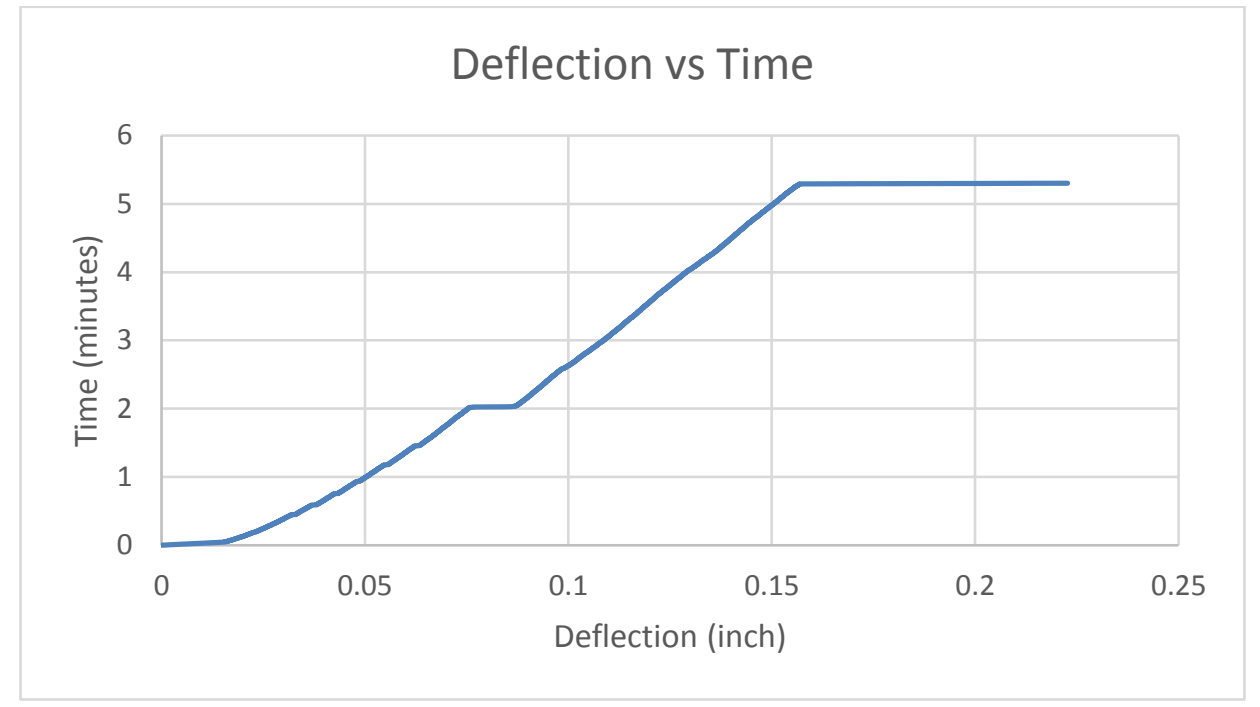

Figure 3-12: Example of deflection over time for pushout tests

\subsection{Pull off Bond Test (Modified ASTM D7522)}

\subsubsection{Test Development}

While the bond testing described in Section 3.3 could provide a value of bond strength in laboratory conditions, evaluating installed FRP systems for proper bonding in the field is equally 
important. For concrete samples, ACI recommends using ASTM D7522 to establish the bond strength of FRP bonded to concrete. To use this test method, a 2 inch inner diameter core drill is used to cut into the FRP and concrete to a depth of $1 / 4$ to $1 / 2$ inch. A 2 inch diameter loading fixture (carrier) is bonded to the FRP using high strength epoxy. After curing, a tension force is applied to the carrier via a tester and the failure load and failure type is documented. However, there are no similar ASTM test procedures for FRP bonded to timber. In light of this, pull off tests were conducted on the FRP systems bonded to creosote treated timber piles using a modified ASTM D7522 to provide a general range of values and to correlate these values to the bond values determined using the method in Section 3.3.

\subsubsection{Specimen Preparation}

Pull off testing specimens consisted of four timber pile portions ( 8 " in height with 9" to 10" diameter) each wrapped with one of the composite wrap systems (Sika, Fyfe, Phenolic, and Aqua Wrap). Five pull offs were used on each system. Before wrapping, the piles were gently sanded to remove loose splitters and to open up the pores of the timber for bonding. The timbers were primed by applying the resin used to saturate the fabric in a thin, even layer around the circumference of the pile. Exception to the priming process was the Aquawrap ${ }^{\circledR}$ preimpregananted system and does not provide a primer resin for application. Three layers of saturated FRP wrap were applied using hand lay up around the pile's circumference. Stricture wrap was applied for all systems with uniform pressure around the wrapped portions to remove voids and prevent layers from experiencing delamination before the composites were fully cured.

After curing for at least 7 days, specimens were prepared for pull off testing. First the composite was drilled with a 2 1/8" composite hole-saw through the FRP. Next a 2 1/8" wood hole saw completed the cut $1 / 4$ " to $1 / 2$ " into the timber. This was done to ensure a localized bond strength 
was being tested and prevent contributions from the rest of the bond area. Consistent cutting was ensured by a small wooden plate with a hole that matched the hole-saw diameter attached with screws as shown in Figure 3-13. The plate allowed the saw to cut in the same location on the specimen without any misalignment. After the holes were drilled and the securing plate removed, aluminum pull off pucks with 2" diameters were attached to the composite with 2500 psi fast setting epoxy and secured by timber sheaths as shown in Figure 3-14.

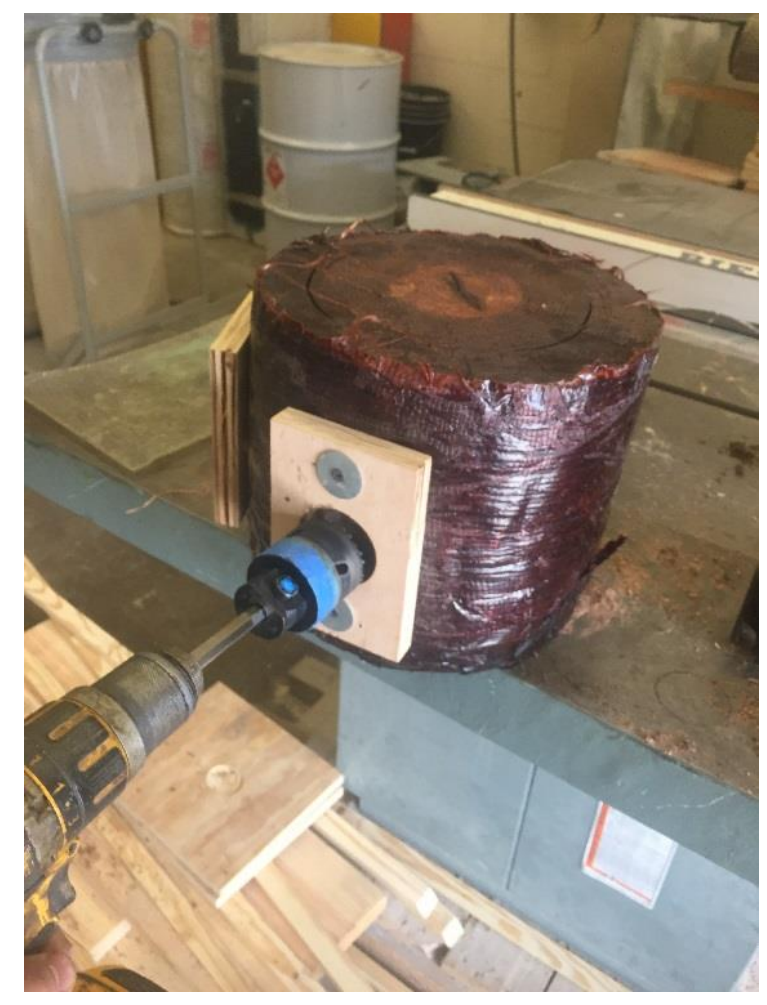

Figure 3-13 : Cutting of timber for pull off tests 


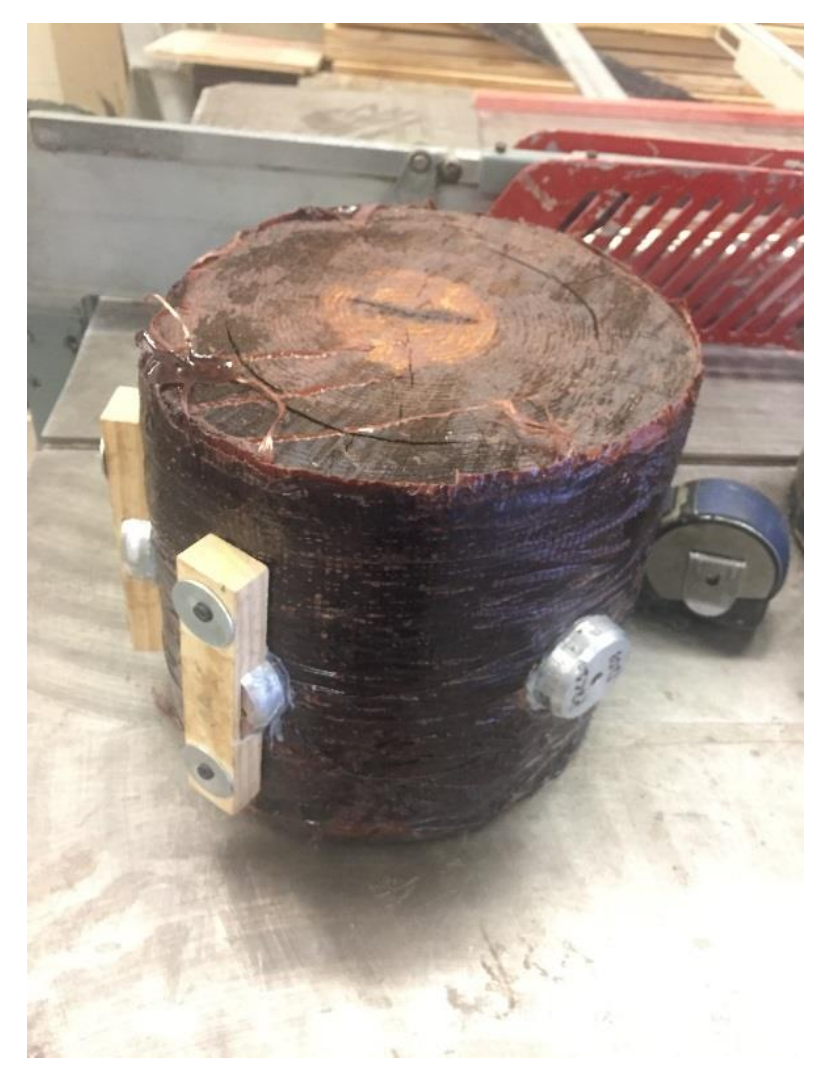

Figure 3-14: Pull off puck attachment technique

\subsubsection{Testing Apparatus}

Dyna Proceq Z16 was the pull off apparatus used to remove the pucks from the specimens. The apparatus consisted of three adjustable legs with a single testing shaft attached to the pucks, adjusted by a hand crank as seen in Figure 3-15. Tests were considered completed when the pull off pucks achieved a peak load resulting from the FRP bond failing or exceeding the wood tensile strength. 


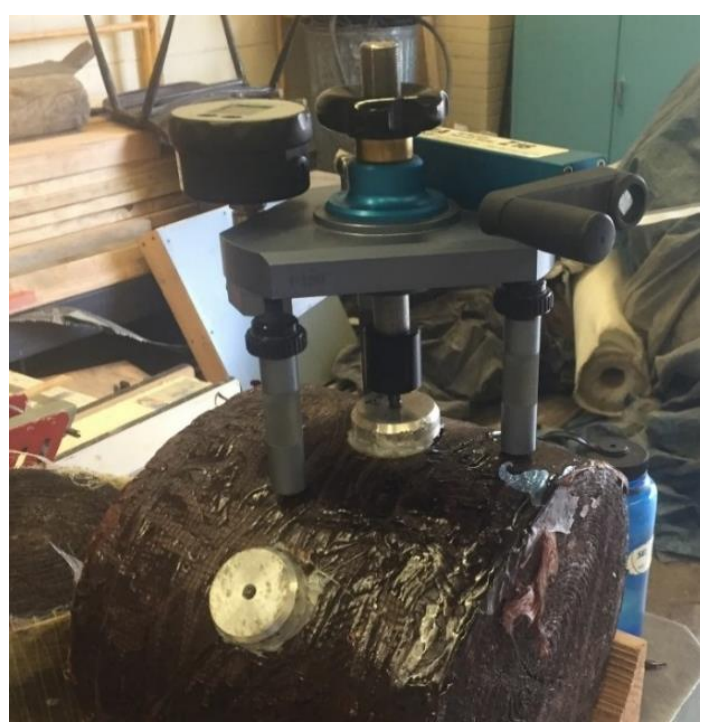

Figure 3-15: Dyna Proceq Z16 pull off tester

\subsection{Compression Testing}

\subsubsection{Compression Test Development}

To determine how to most efficiently evaluate the full sized (i..e. 9 inch diameter and 24 inch long) shells in compression, preliminary testing was conducted on 6 inch diameter, 10 inch long compression samples as included in the Appendix B. These preliminary tests revealed that the samples tended to fail in bearing where the FRP material was in contact with the hardened steel testing platen. Several different methods of reinforcing the ends of the test samples were evaluated, but it was found that the simplest and most effective method was to provide at least three strips of FRP, effectively doubling the wall thickness for the top and bottom 6" of the samples.

Manufacturing methods for creating the shells were also developed through experimentation. Initially, specimens were found to be sticking to the PVS pipes used as molds after curing, and mold release agents were not found to be effective for the full size samples although they were effective for smaller samples. As a solution, two layers of $3 \mathrm{~mm}$ plastic sheeting 
were placed around the molds allowing the shell to cure to one of the plastic layers while sliding off of the second underneath. Additionally initial shells were found to shrink onto the molds as they cured, requiring over $20,000 \mathrm{lbs}$ of force to remove the mold from inside the shells. To address these issues, PVC pipes were slit lengthwise. The top of the molds were held open by set screws that were used to expand the molds and insert a $1 / 2$ " wide wood strip into the lengthwise cut in the pipe, thus expanding the diameter of the PVC pipe as shown in Figure 3-16. After the FRP was wrapped and cured on the mold, the wooden strips were removed the PVC mold snapped back to their original shape and could easily be removed. After these adjustments, successful shells for compression testing could be produced.

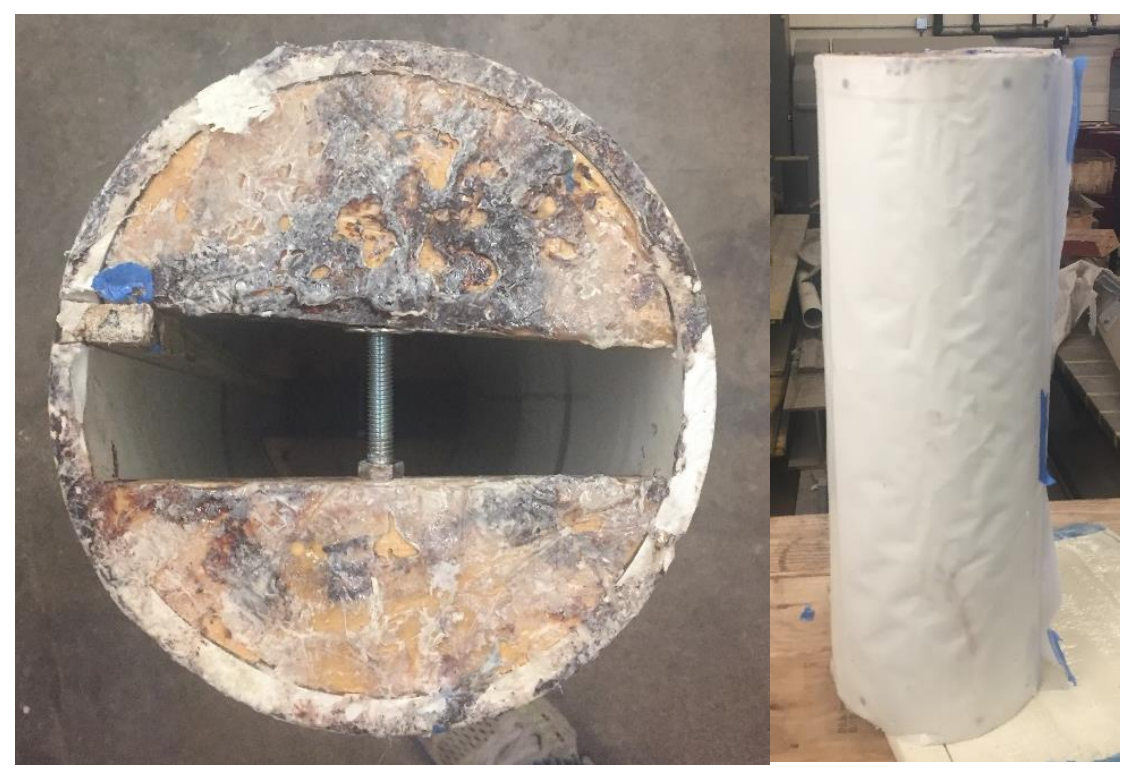

Figure 3-16: PVC Mold for compression testing

\subsubsection{Specimen Preparation}

To evaluate compression, wet layup tube specimens were manufactured consisting of both three and five layers (three of each tube type). The tubes were manufactured on PVC molds to be roughly 9" in diameter and 24" in height. For Fyfe, Sika, and Phenolic, fabric was cut to 30 inch in width and 24 inch in height for a single layer. During wrapping, the resins were prepared as per 
the specifications and applied to fabric in order to saturate them fully as shown in Figure 3-17. For unidirectional Fyfe and Sika fabric systems, the layers were oriented with the majority of fibers in the longitudinal directions in a [0/90/0] orientation for three layers and [0/90/0/90/0] for five layers of wrap. For the bidirectional fabric systems (Aquawrap® and Phenolic), the wraps were simply wrapped with three and five layers as necessary. See Figure 3-18 for example of wrapping shells.

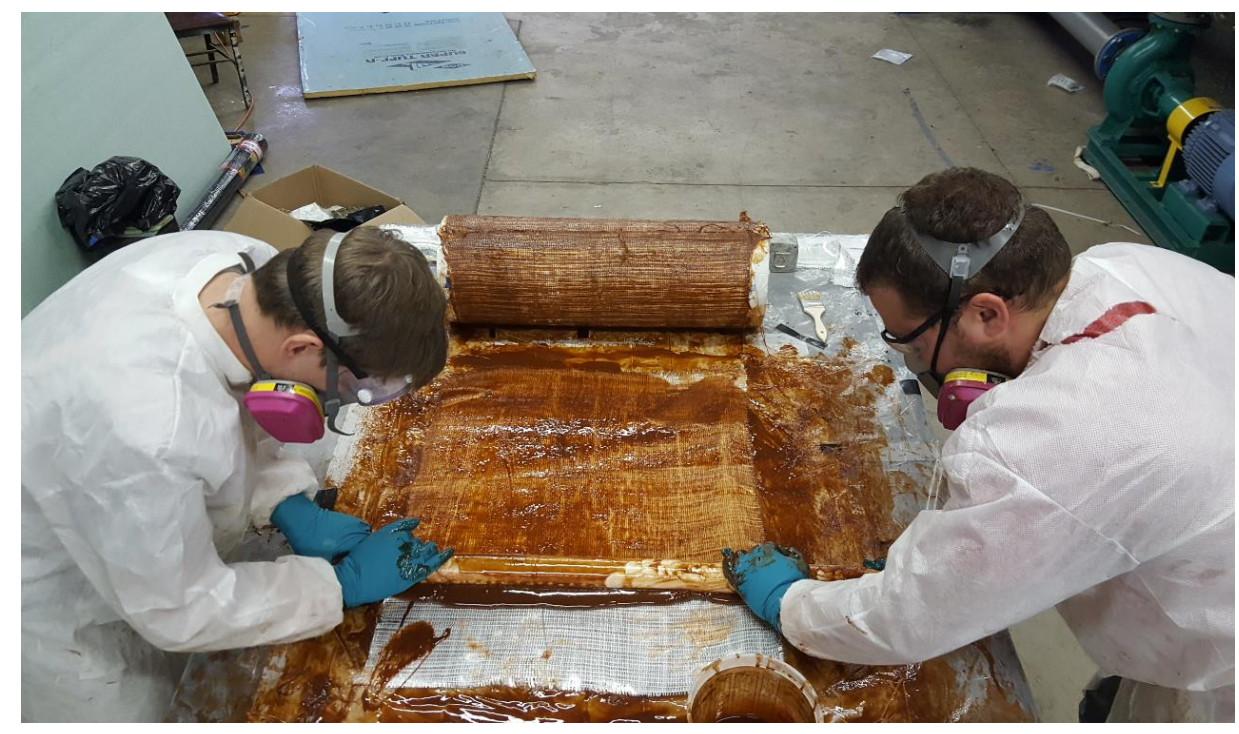

Figure 3-17: Saturating fabric and spreading resin

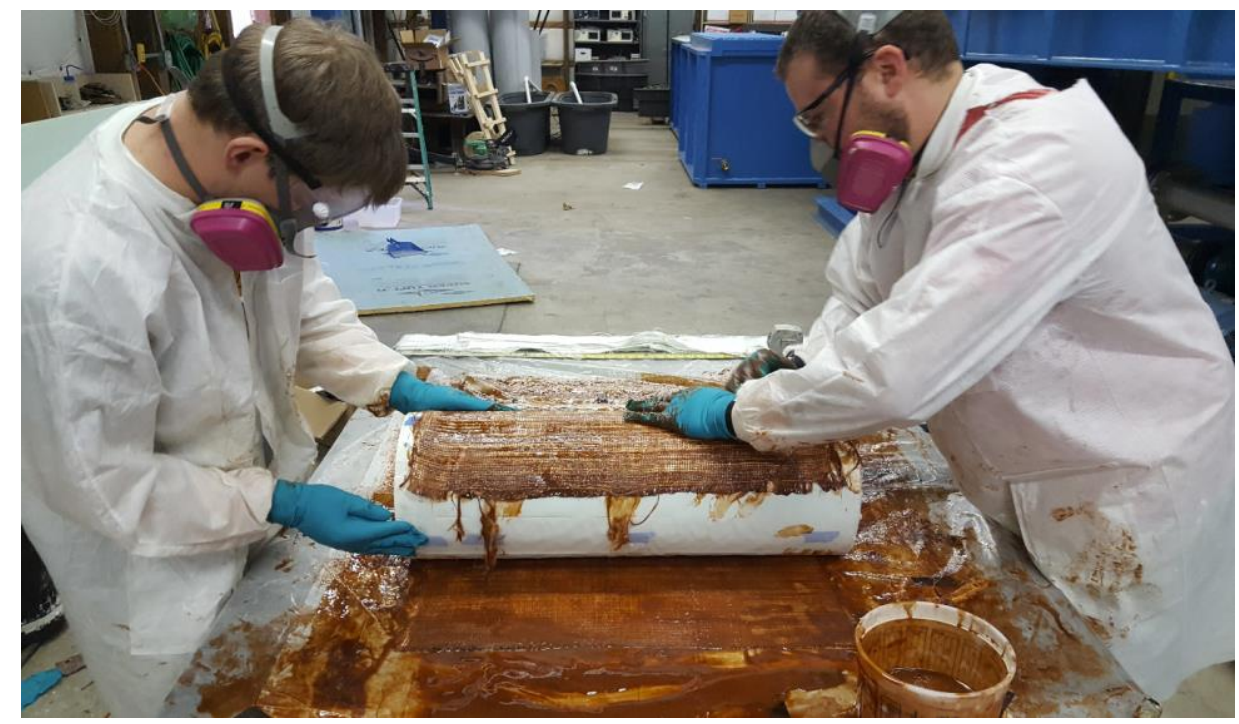

Figure 3-18: Wrapping of compression test tubes 
For the Aquawrap®, 12 inch wide rolls were provided. The first and third layers were applied by laying 12 inch portions next to each to create a 24 inch portion. The second layer consisted of one 12 inch portion in the middle and two 6" portions on either side. The same process was used with the five layer specimens.

To prevent fiber crushing on top and bottom of the shells during compression testing, additional strips were placed on the top and bottom with a width varying from 4" to 6". The strips do not interfere with the compressive capacity of the shells during testing but instead function similar to grips used in tension testing of coupon samples. After applying the extra reinforcement, the specimens were wrapped with stricture wrap to ensure proper bonding between layers and to keep the specimens from slipping off the forms. Specimens were wrapped and cured above 70 degrees F. After curing the specimens, the ends of the specimens were cut square to provide a smooth bearing specimen for uniform load distribution, preventing stress concentrations. All completed shells are shown in Figure 3-19. Witness panels for each system were also created with three layers of wrap to be later cut into coupons and tested for tensile capacity. Coupons were made by building up the wrap in layers on a steel table covered in plastic. After all three layers were built up, a second layer of plastic was applied and a board placed on top of the panel. Weights were placed on the board to apply a uniform curing pressure. Coupons are shown below for Fyfe (Figure 3-22), Sika (), Aquawrap® (Figure 3-23), and Phenolic (Figure 3-20). 


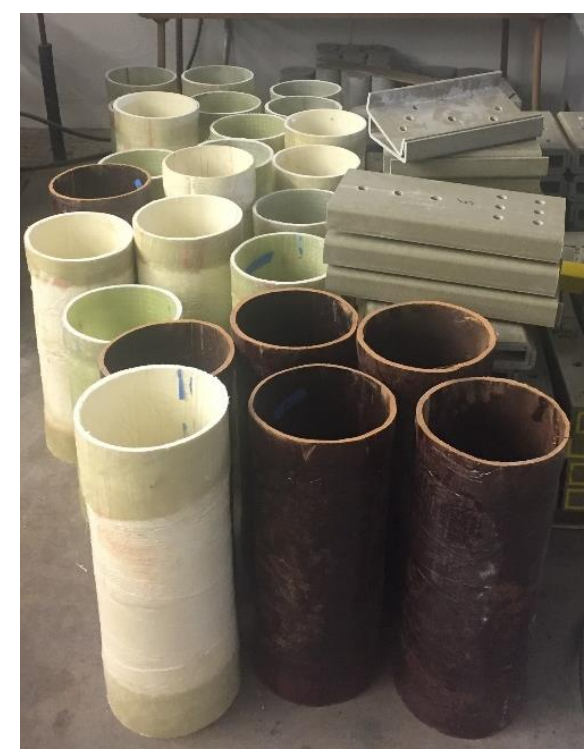

Figure 3-19: FRP Shell Specimens

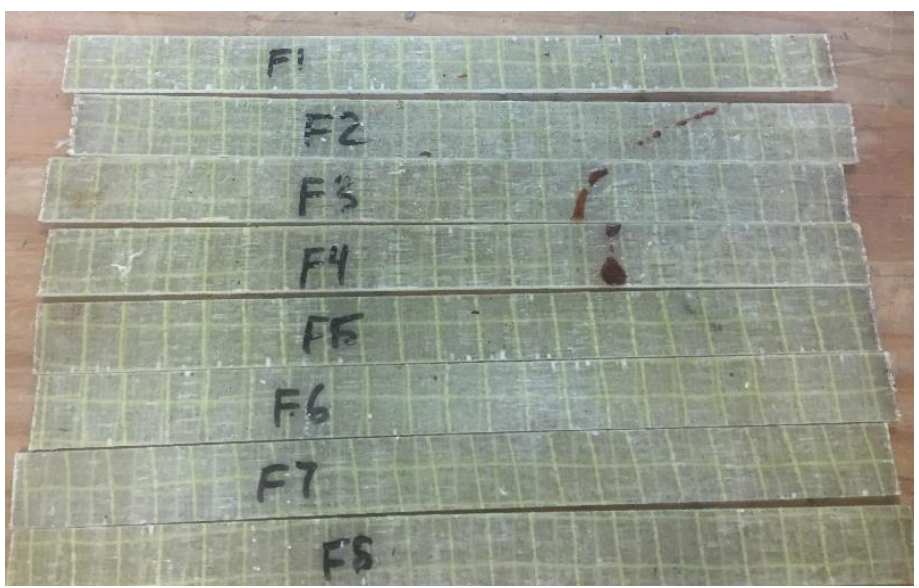

Figure 3-20: Fyfe Coupon Samples 


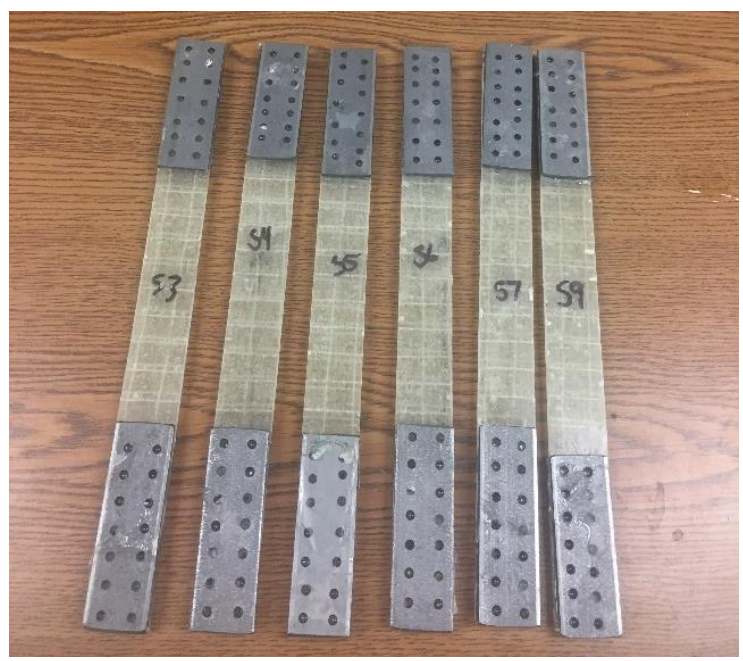

Figure 3-21: Sika Coupons

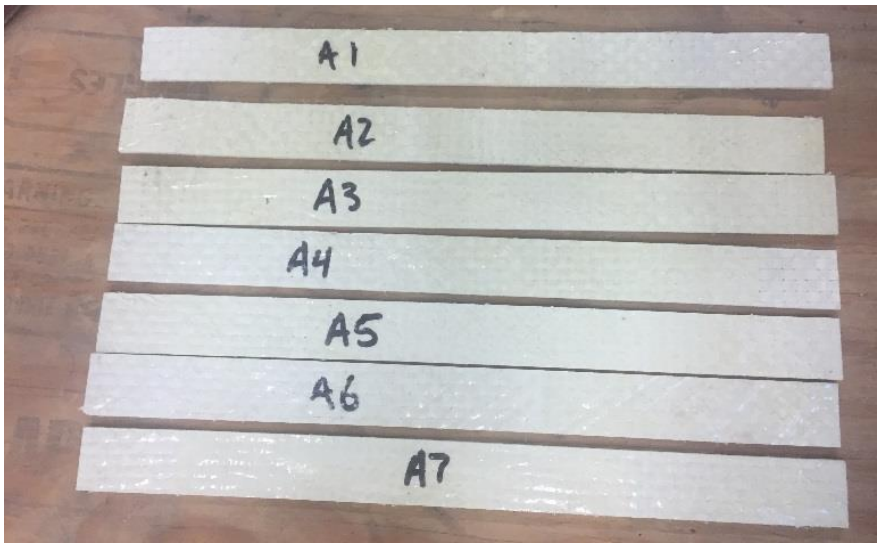

Figure 3-22: Aquawrap ${ }^{\circledR}$ Coupon Samples

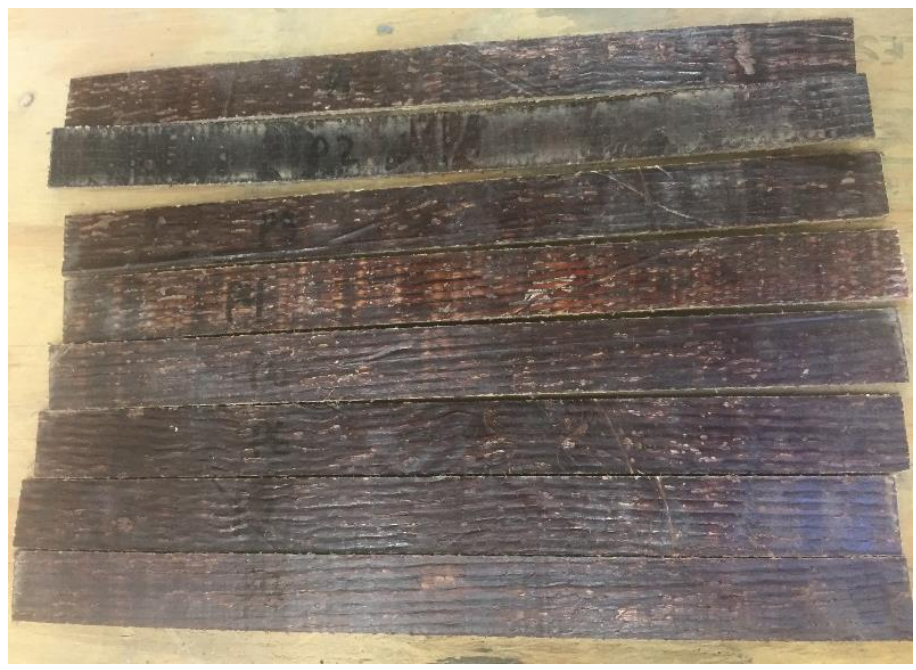

Figure 3-23: Phenolic Coupon Samples 


\subsubsection{Testing Apparatus}

The same Instron machine was used for bond testing was used for the compression testing, with loading rates adjusted to achieve failure in 2 to 10 minutes. Loading rate for Sika and Fyfe system was 20 kips per minute. For the Phenolic system, load rates were $20 \mathrm{kips} / \mathrm{min}$ (A and B). 5 kip/min (Phenol C and D), and $10 \mathrm{kip} / \mathrm{min}$ (Phenol E \& F). For the Aquawrap® shells, loading rates were $20 \mathrm{kip} / \mathrm{min}$ (Aqua A) and $10 \mathrm{kip} / \mathrm{min}$ (Aqua B-F). 2" steel plates were used to supply a large bearing surface on the test machine. For the configuration, see Figure 3-24

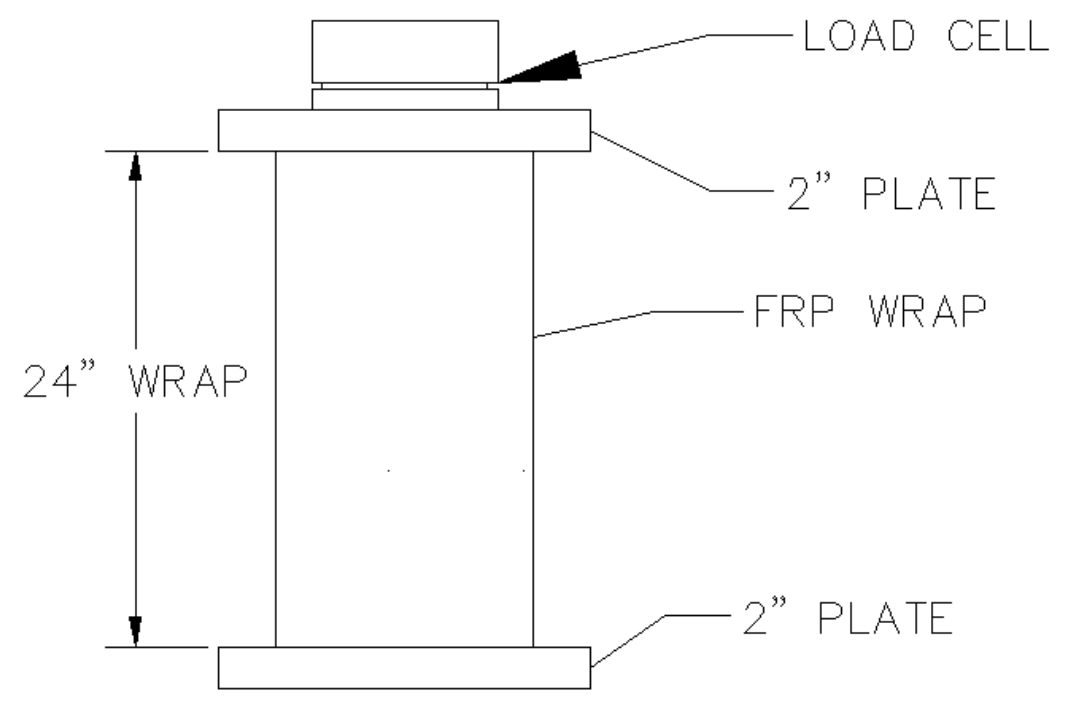

Figure 3-24: Schematic of compression test

Failure occurred after fiber crushing or laminate de-bonding. An example of the fiber failure when plotted is shown in Figure 3-25. The drop after maximum indicates the failure. See the arrow that is used to represent the peak. 


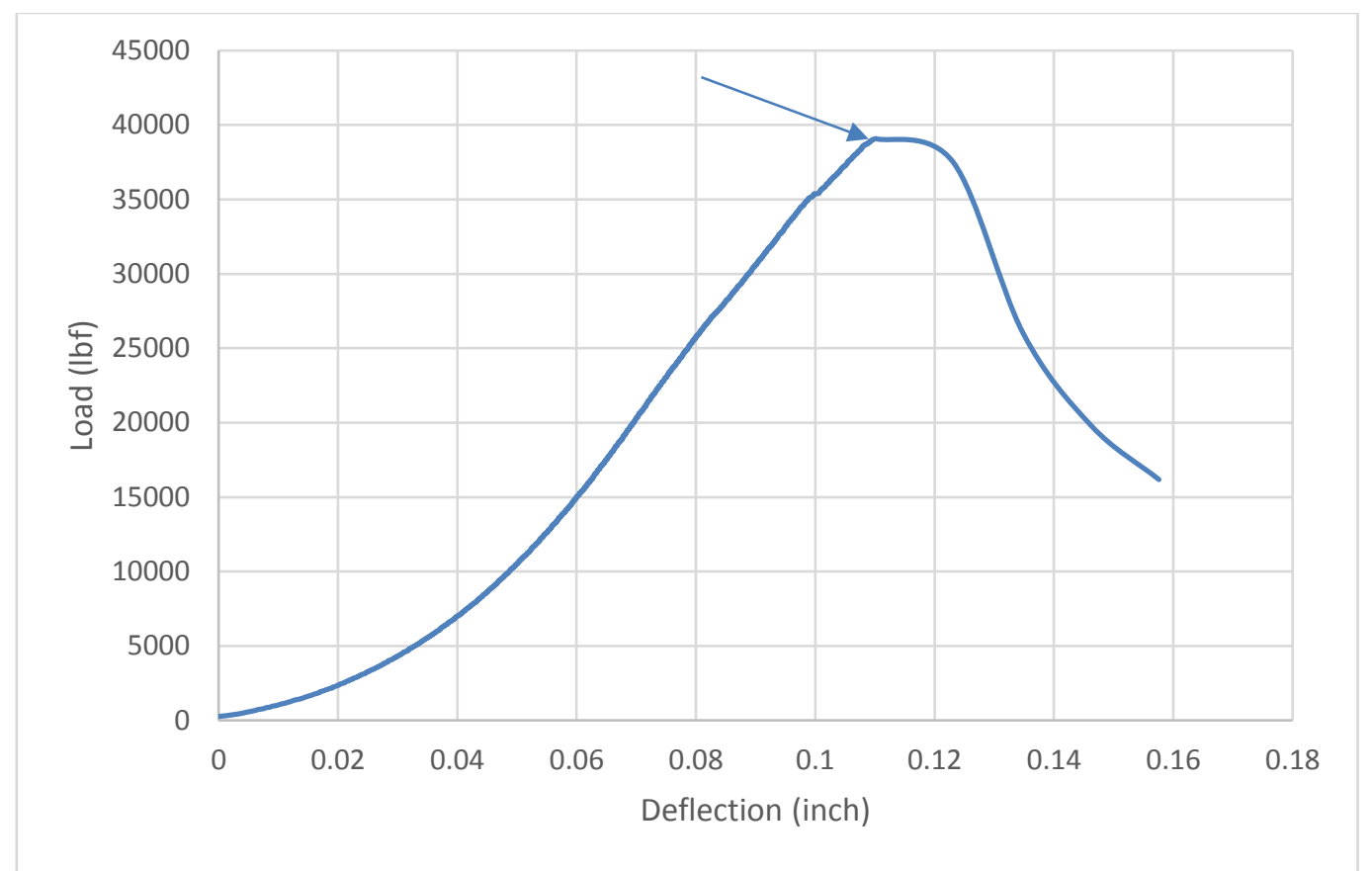

Figure 3-25: Example of compressive loading

Coupons were tested on an Instron 8501 tensile machine (Figure 3-26) at a loading rate of $.05 \mathrm{in} / \mathrm{min}$ until failure as per ASTM D3039 with no conditioning or post-curing of the coupons.

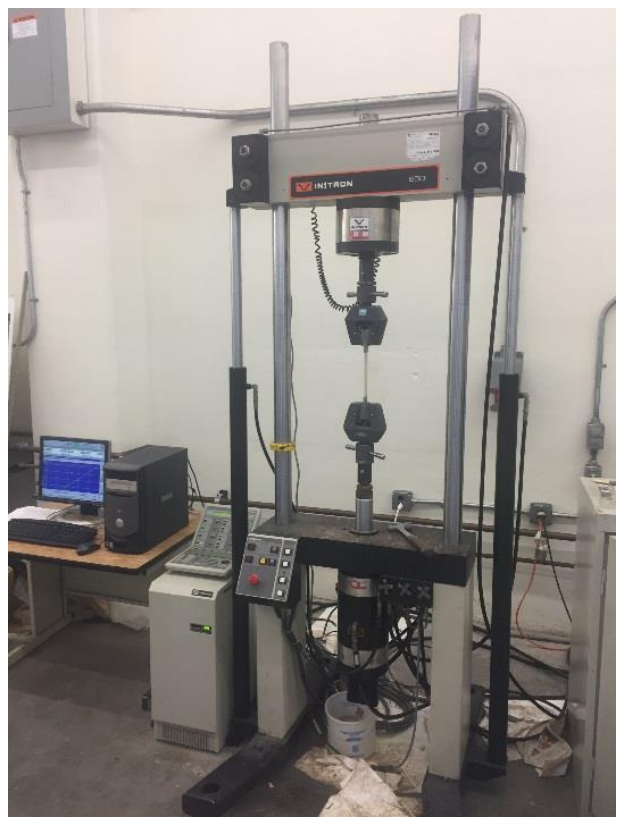

Figure 3-26: Instron 8501 


\subsection{Full Scale Rehabilitation Simulation}

\subsubsection{Rehabilitation Simulation Test Development}

Once the bond strength and compression capacities of the systems were established separately, it was determined that evaluating the two failure modes together was needed to demonstrate rehabilitation capacities that might be encountered in the field. Because the capacity of decayed timber in the field is uncertain and difficult to determine, the deteriorated timber portion cannot be counted upon to provide any reasonable capacity and should be neglected. Therefore, evaluations of the compressive and bond capacity of the wrap system should be evaluated in tandem while excluding the decayed timber. To accomplish this two 16" timber sections separated by a 18" gap were wrapped with each FRP system and tested under axial compression.

\subsubsection{Specimen Preparation}

To ensure the timber samples remained parallel to each other during testing, a steel pipe was used for alignment and to aid in handling. The two 16 " portions of timber pile were drilled with a 2" vertical hole in order to place it on the 2" steel pipe. The ends were secured with timber plates to prevent slipping and ensure alignment as shown in Figure 3-27. 


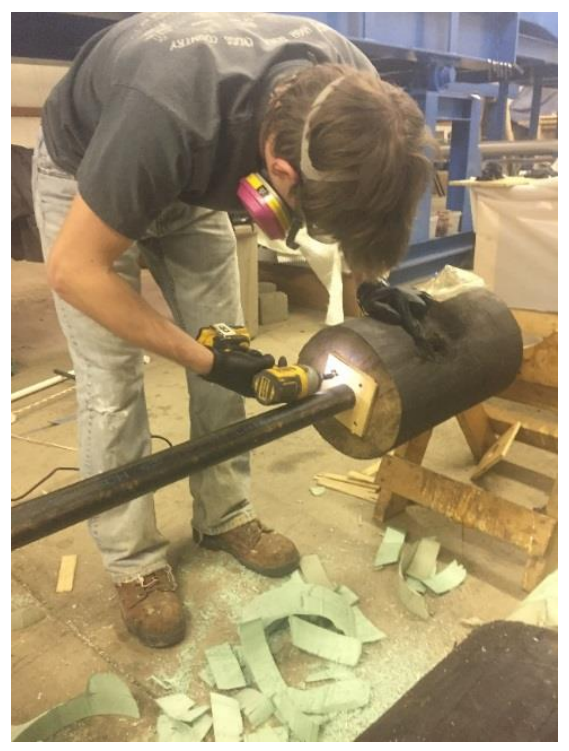

Figure 3-27: Setting timber for rehabilitation

The two portions were separated by an 18 " gap created by 9 pieces of 12 " x 12 " x 2 " extruded insulation. The insulation was then trimmed with a hot wire to match the shape of the timber it was sandwiched between as shown in Figure 3-28. See Figure 3-29 for pictures of the trimmed specimen.

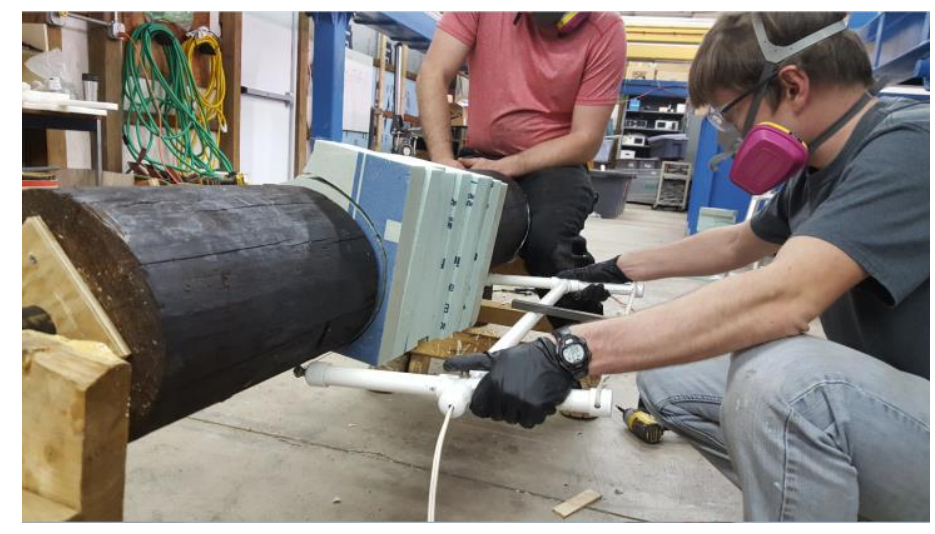

Figure 3-28: Trimming insulation for simulated rehabilitation 


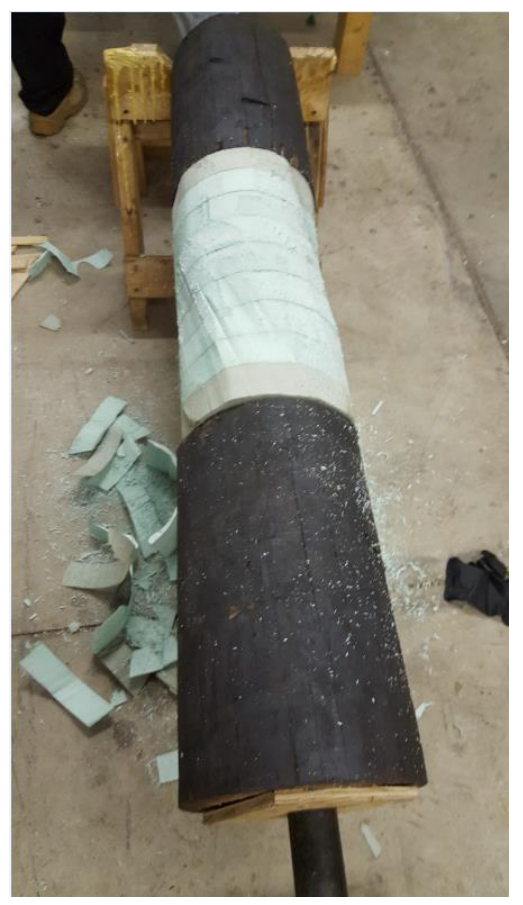

Figure 3-29: Simulated rehabilitation specimen

Dry wall compound was utilized to fill any voids developed during the trimming process and provide a uniform wrapping surface. The timber specimens were then wrapped with the FRP wraps as shown in Figure 3-30. For the hand lay-up specimens (Fyfe, Phenolic), fabrics were cut to 42 " in the longitudinal by 38 " in the hoop direction. After curing for 7 days, the pipes were removed from the specimens, and the insulation was melted away with acetone to produce a hollow core between the timber pieces. 


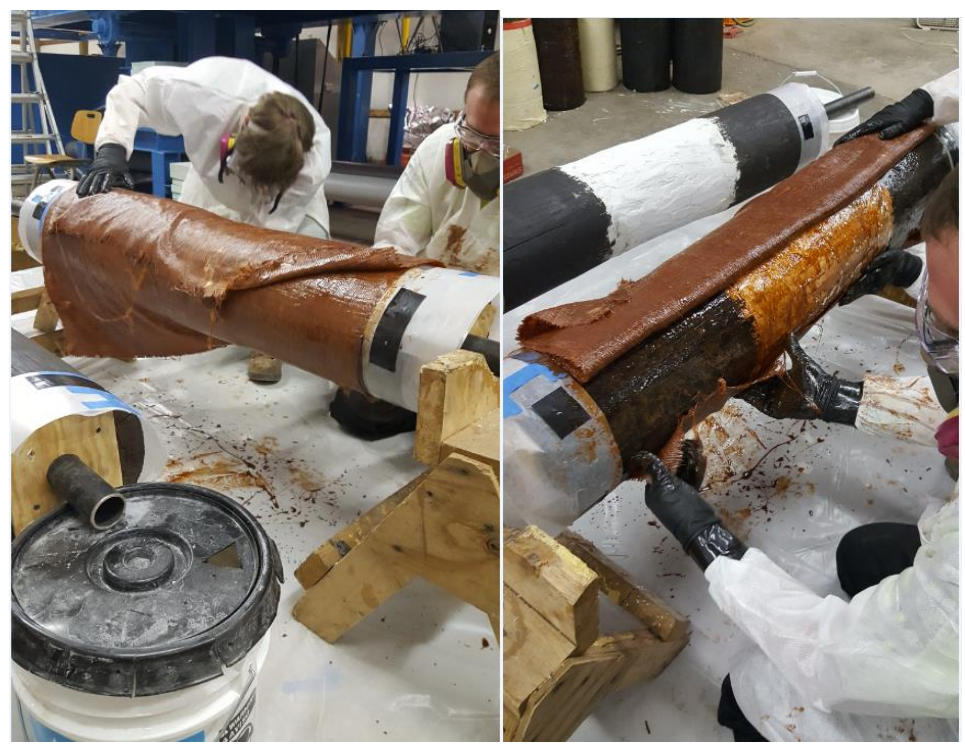

Figure 3-30: Wrapping of rehabilitation specimens

\subsubsection{Testing Apparatus}

The configuration of the simulated rehabilitation testing is shown in Figure 3-31. Instron 1000HDX universal testing was used as before as shown in Figure 3-32. Failure was determined by either bond slippage or compressive failure in the wrap. Explanations for determining these failures were mentioned above in Sections 3.3 and 3.5. Loading was applied at a constant rate of $10 \mathrm{kip} / \mathrm{min}$ until failure occurred in the specimens. 


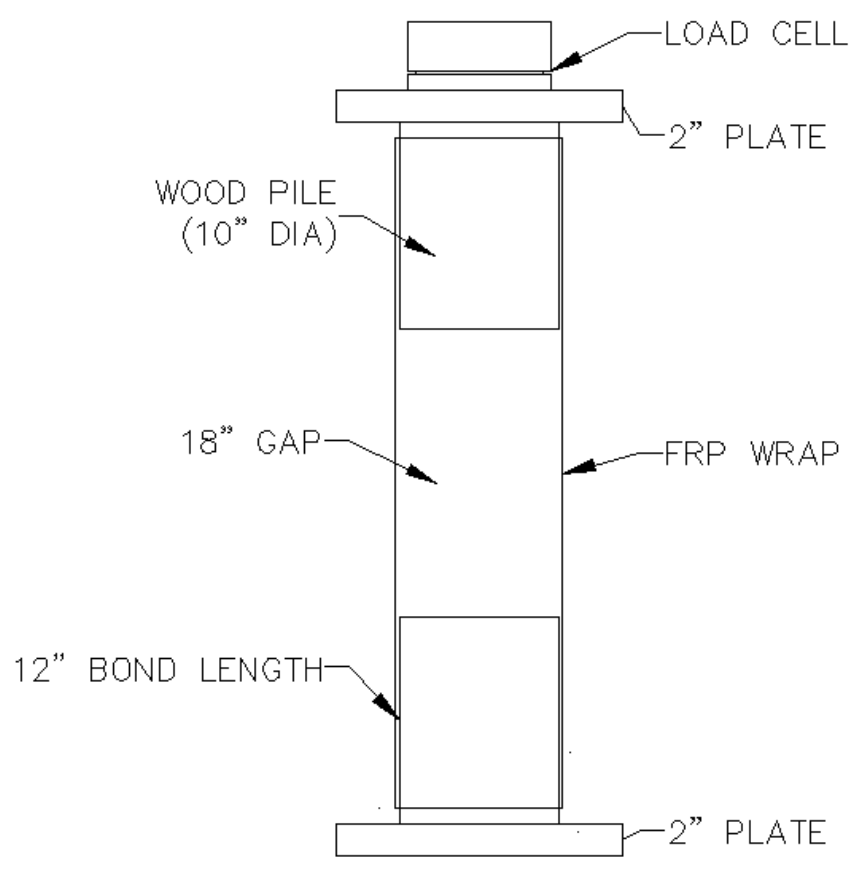

Figure 3-31: Schematic of Simulated Rehabilitation Test

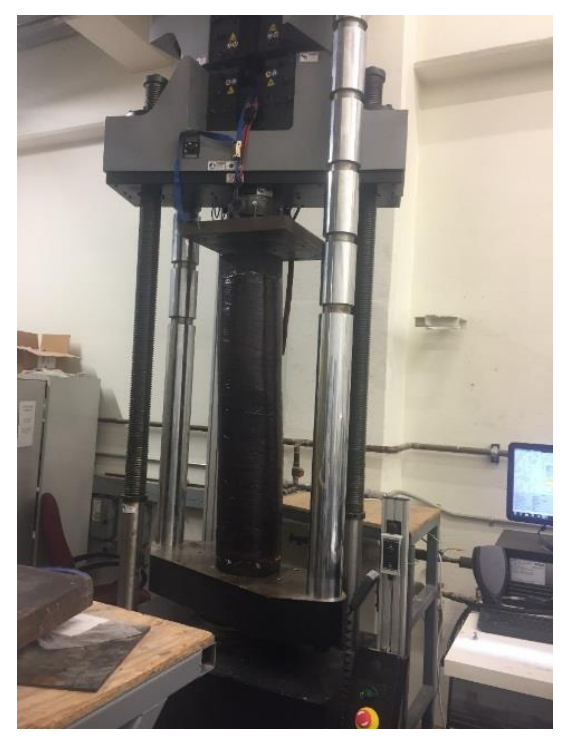

Figure 3-32: Simulated rehabilitation evaluation testing setup 


\section{CHAPTER 4 DATA ANALYSIS AND DISCUSSION}

\subsection{Introduction}

The data from the evaluations are presented for each system with very limited discussion of behavior except to note any unusual behavior in the system. Presented data includes maximum values and visual inspections of wraps after testing. Following results, discussions of the failure mechanisms and significance of the results for each of the tests are provided for each test type. Results are divided into Sections 4.2 and 4.3 for bond, Section 4.4 for compression, and Section 4.5 for simulated timber rehabilitation evaluations.

\subsection{Bond Testing (Push out)}

The bond pushout testing was conducted on the four systems for 6" and 12" bond lengths. Results are presented in this section for Fyfe (4.2.1), Sika (4.2.2), Aquawrap® (4.2.3), and Phenolic (4.2.4). Discussions of the results of the bond evaluations are provided in 4.2.5.

\subsubsection{Fyfe Bond Strengths}

\subsubsection{Capacity and strength of Fyfe/timber bonds}

All Fyfe/timber bond specimens failed in bond slippage as additional reinforcement was applied around the base to prevent fiber crushing. Average bond strengths for 6" and 12" of lengths of bond were 281 psi and 240 psi. Capacities were 61.2 kips and 90.5 kips for 6" and 12" bond lengths respectively. Results from the bond evaluations are reported for the 6" and 12" Fyfe bond lengths are shown in Table 4-1 and Table 4-2. In the case of sample FY6-3 (ID 6P2-2), a gap 
between the wrap and the timber was noticed at the top of the specimen which seems to have influenced the bond capacity; therefore this result was excluded from the average calculations.

Table 4-1: Fyfe 6" Bond Length Results

\begin{tabular}{|c|c|c|c|c|}
\hline Sample & ID & $\begin{array}{l}\text { Bond } \\
\text { Area } \\
\left(\text { in`2 }^{\wedge}\right)\end{array}$ & $\begin{array}{c}\text { Peak } \\
\text { Load } \\
\text { (lbf) }\end{array}$ & $\begin{array}{c}\text { Peak } \\
\text { Stress } \\
\text { (psi) }\end{array}$ \\
\hline FY6-1 ${ }^{1}$ & $6 \mathrm{P} 1-5$ & 198 & 53,233 & 269 \\
\hline FY6-2 ${ }^{1}$ & $6 \mathrm{P} 2-3$ & 191 & 69,067 & 362 \\
\hline FY6-3 ${ }^{1}$ & $6 \mathrm{P} 2-2$ & 198 & 41,853 & 211 \\
\hline \multirow{2}{*}{\multicolumn{3}{|c|}{$\begin{array}{r}\text { Average: } \\
\text { COV: }\end{array}$}} & 61,150 & 315.5 \\
\hline & & & $18 \%$ & $21 \%$ \\
\hline
\end{tabular}

Table 4-2: Fyfe 12” Bond Length Results

\begin{tabular}{|c|c|c|c|c|}
\hline Sample & ID & $\begin{array}{l}\text { Bond } \\
\text { Area } \\
\left(\text { in^}^{\wedge} 2\right)\end{array}$ & $\begin{array}{c}\text { Peak } \\
\text { Load } \\
\text { (lbf) }\end{array}$ & $\begin{array}{c}\text { Peak } \\
\text { Stress } \\
\text { (psi) }\end{array}$ \\
\hline FY12-1 ${ }^{1}$ & $12 \mathrm{P} 3-4$ & 363 & 75,718 & 209 \\
\hline FY12-2 1 & $12 \mathrm{P} 2-5$ & 391 & 97,572 & 249 \\
\hline FY12-3 ${ }^{1}$ & $12 \mathrm{P} 1-4$ & 377 & 98,333 & 261 \\
\hline \multirow{2}{*}{\multicolumn{3}{|c|}{$\begin{array}{r}\text { Average: } \\
\text { COV: }\end{array}$}} & 90,541 & 240 \\
\hline & & & $14 \%$ & $11 \%$ \\
\hline
\end{tabular}

\subsubsection{Visual inspection of Fyfe/Timber bonds after testing}

After testing, 6" and 12" wraps were cut from the test samples to examine the wrap for retained material. As shown in both Figure 4-1 and Figure 4-2, delaminated wraps contained large portions of timber (1/4" to $1 / 2$ " in thickness). Such behavior indicated failure of the timber substrate and not the bond (failure of the resin matrix). From this visual evaluation, failure in the timber is assumed to be the controlling failure mode for the majority of the Fyfe bond specimens. 


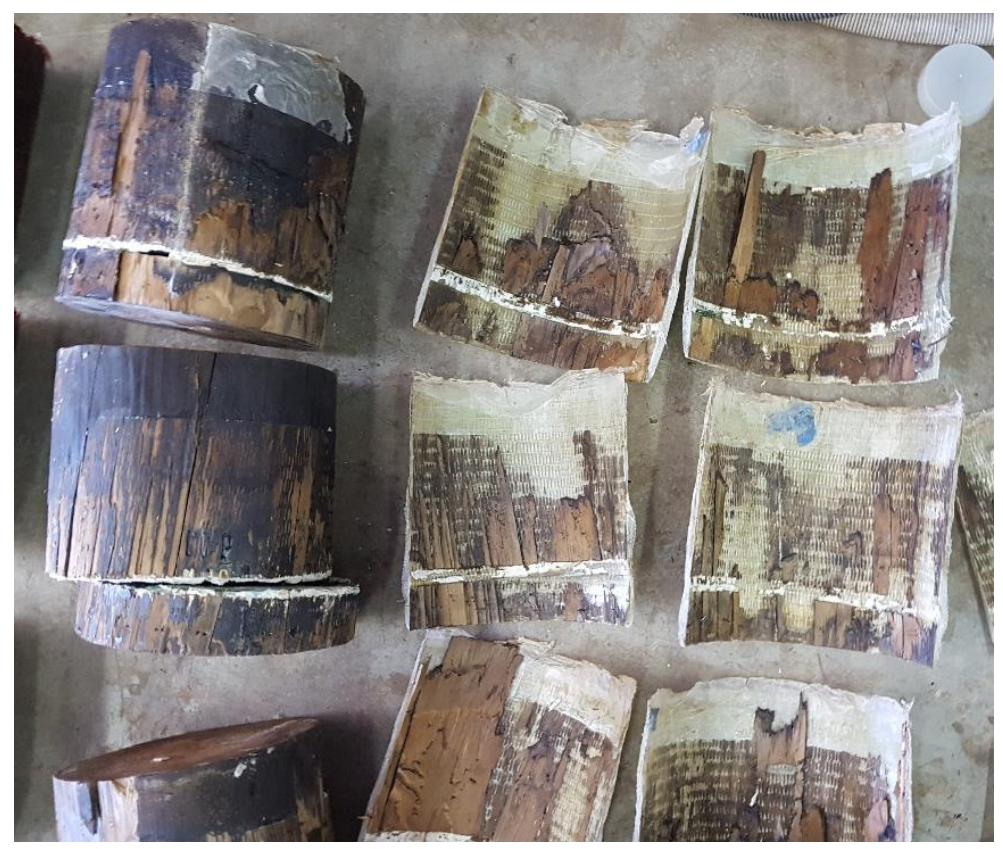

Figure 4-1: Retained timber on Fyfe 6" bond lengths

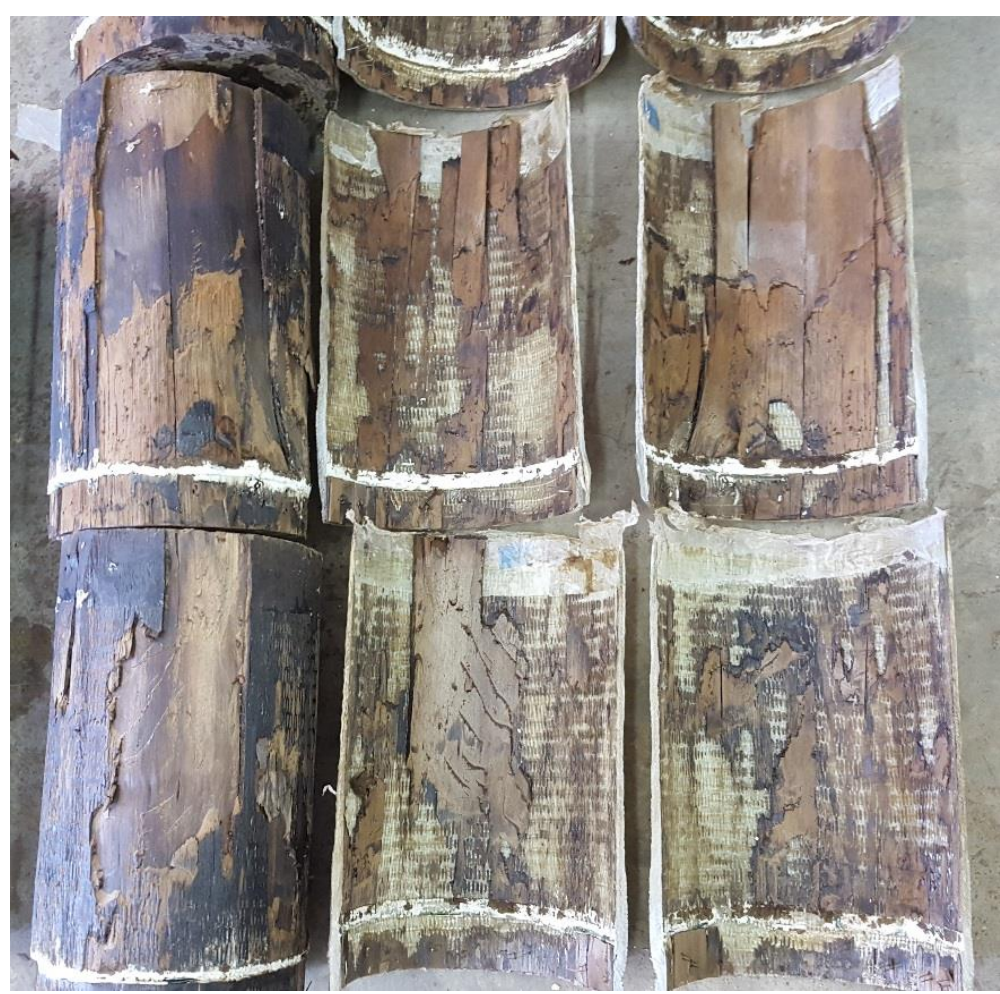

Figure 4-2: Retained timber on Fyfe 12" bond lengths 


\subsubsection{Plot of load vs deflection of Fyfe pushout}

The load versus deflection plots shown in Figure 4-3 correlate well with the assumption of failure in the timber. The sharp drops in capacity coupled with rapid deformation shown on several of the specimens would seem to indicate the ripping failure in the timber. More testing would be needed to verify this assumption. The figure also shows the lowered capacity of the 6" bond specimen (dashed purple line).

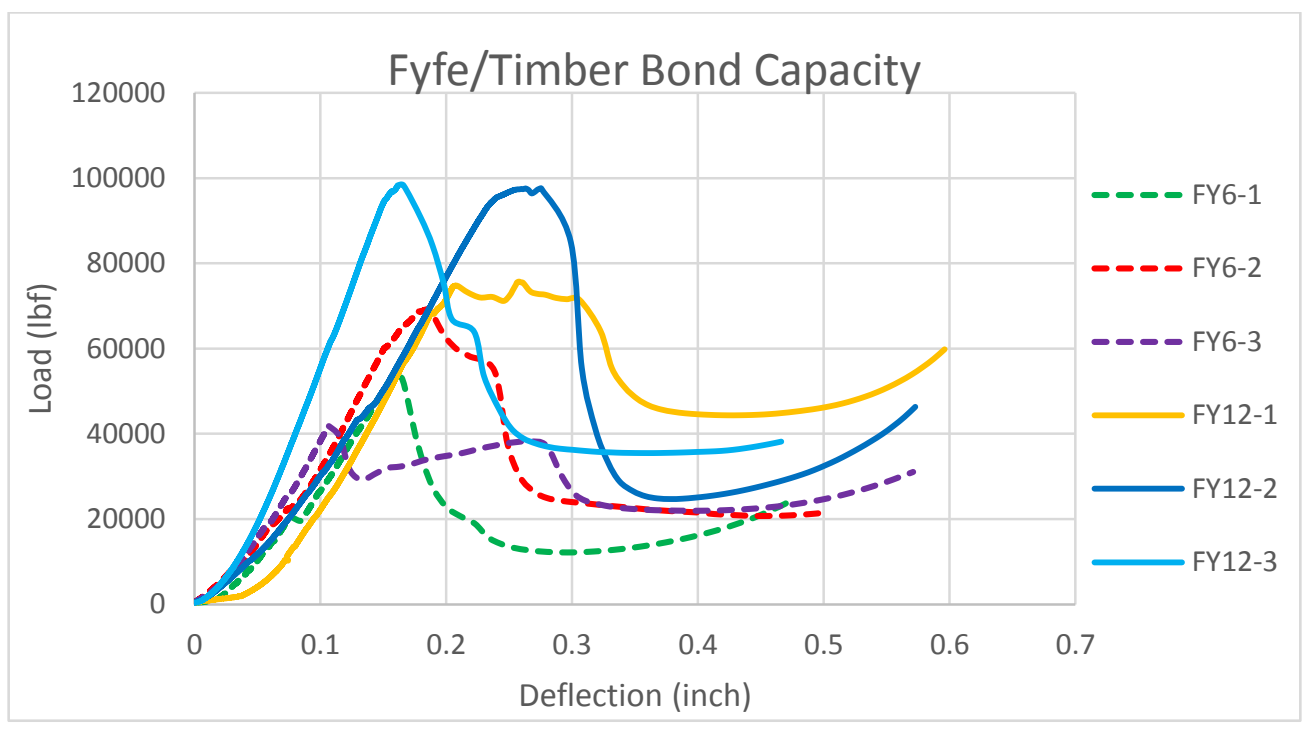

Figure 4-3: Fyfe/timber bond capacity (lbf)

\subsubsection{Sika Bond Strengths}

Due to a supply issue, the Sika/timber bond capacities were not evaluated at the time this. However due to the similarities between the Sika and Fyfe systems (same fabric type/epoxy resin), it is predicted that results similar to those obtained in the Fyfe/timber bond evaluations will be obtained. 


\subsubsection{Aquawrap® Bond Strengths}

\subsubsection{Capacity and strength of Aquawrap ${ }^{\circledR} /$ Timber bonds}

Failure modes varied from bond slippage for the 6 inch samples to crushing of the fibers in the base for the 12 inch samples as the Aquawrap ${ }^{\circledR}$ specimens were not reinforced in the base as were the other systems. Average bond strengths for 6" and 12" of lengths of bond were 82 psi and 56 psi respectively. When bonded to a 10" diameter timber, 6" bond lengths provided an average capacity of approximately 20.4 kips while 12 " bond lengths carried around 22.3 kips for the same approximate diameter. Results from the bond evaluations are reported for the 6" and 12 " Aquawrap ${ }^{\circledR}$ bond lengths are shown in Table 4-3 and Table 4-4. Failure modes are indicated by subscripts on the tables. Due to its comparatively low value, sample AQ6-2 was considered an outliner and neglected from the average values calculated.

Table 4-3: Aquawrap ${ }^{\circledR}$ 6” bond lengths

\begin{tabular}{|c|c|c|c|c|}
\hline Sample & ID & $\begin{array}{l}\text { Bond } \\
\text { Area } \\
\left(\text { in^}^{\wedge} 2\right)\end{array}$ & $\begin{array}{l}\text { Peak } \\
\text { Load } \\
\text { (lbf) }\end{array}$ & $\begin{array}{c}\text { Peak } \\
\text { Stress } \\
(\mathrm{psi}) \\
\end{array}$ \\
\hline AQ6-1 ${ }^{1}$ & 6P1-1 & 219 & 22,783 & 104 \\
\hline AQ6-21 & $6 \mathrm{P} 1-6$ & 200 & 9,803 & 49 \\
\hline AQ6-31 & $6 \mathrm{P} 2-4$ & 192 & 17,921 & 93 \\
\hline \multirow{2}{*}{\multicolumn{3}{|c|}{$\begin{array}{r}\text { Average: } \\
\text { COV: }\end{array}$}} & 20,352 & 98.5 \\
\hline & & & $17 \%$ & $8 \%$ \\
\hline
\end{tabular}

${ }^{1}$ Bond slippage

Table 4-4: Aquawrap® 12" bond lengths

\begin{tabular}{|c|c|c|c|c|}
\hline Sample & ID & $\begin{array}{l}\text { Bond } \\
\text { Area } \\
\left(\text { in`2) }^{\wedge}\right.\end{array}$ & $\begin{array}{c}\text { Peak Load } \\
\text { (lbf) }\end{array}$ & $\begin{array}{c}\text { Peak } \\
\text { Stress } \\
(\mathrm{psi})\end{array}$ \\
\hline AQ12-12 & $12 \mathrm{P} 1-1$ & 409 & 28,412 & 69 \\
\hline AQ12-2² & $12 \mathrm{P} 2-2$ & 373 & 16,394 & 44 \\
\hline AQ12-33 & $12 \mathrm{P} 3-3$ & 401 & 22,202 & 55 \\
\hline \multirow{2}{*}{\multicolumn{3}{|c|}{$\begin{array}{r}\text { Average: } \\
\text { COV: }\end{array}$}} & 22,336 & 56 \\
\hline & & & $27 \%$ & $22 \%$ \\
\hline
\end{tabular}

${ }^{2}$ Fiber crushing in gap/ ${ }^{3}$ fiber crushing in base 


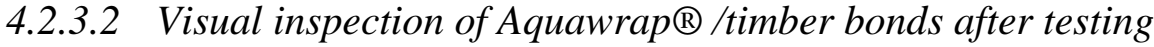

Evaluating the removed wraps after bond testing revealed that limited timber remained as shown in Figure 4-4 on the wrap suggesting that failures occurred in the bond line and not the wood. Failure in the bond line indicates a poor bond strength. Even on one specimens that failed in crushing, only a small sliver of timber was retained as shown in Figure 4-5. Such behavior indicated

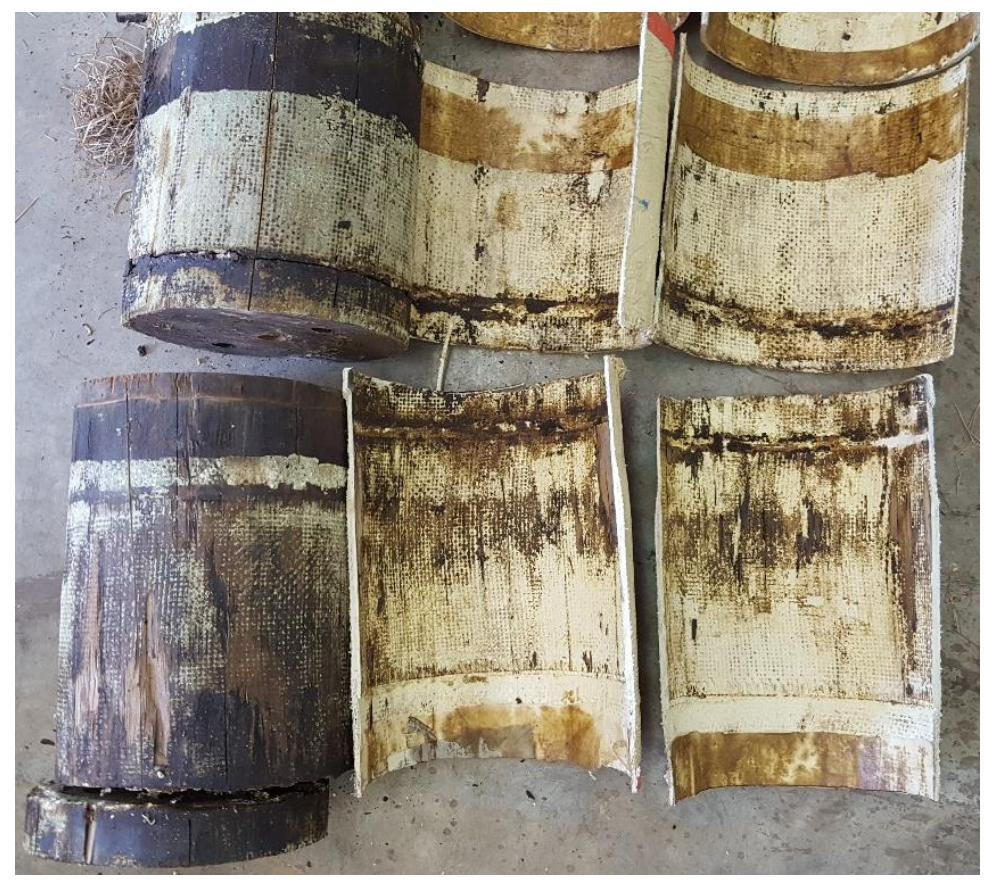

Figure 4-4: Limited retained timber on Aquawrap ${ }^{\circledR}$ 6" and 12" bond specimens

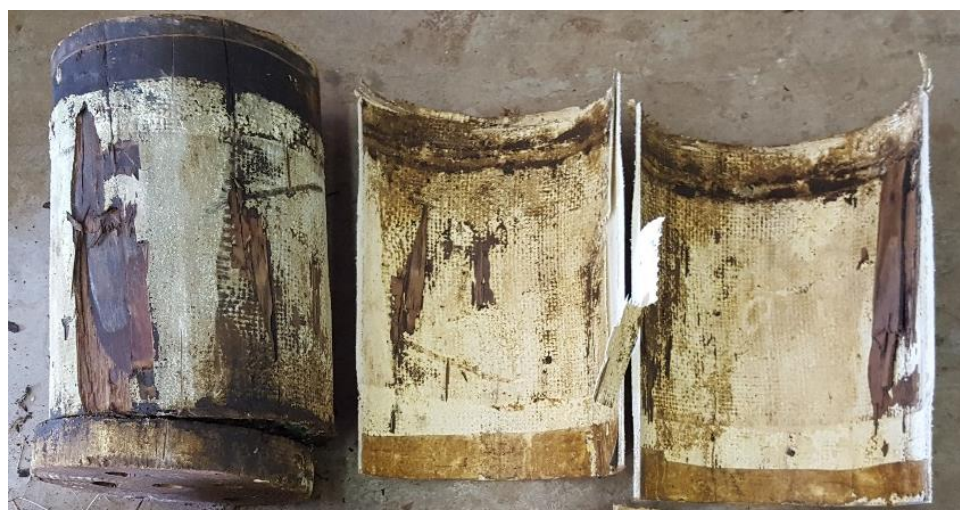

Figure 4-5: Retained timber on Aquawrap ${ }^{\circledR} 12$ " specimen with sliver of timber 


\subsubsection{Plot of load vs deflection of Aquawrap ${ }^{\circledR}$ pushout}

The plots of the Aquawrap bond samples (Figure 4-6) showed extended deflections under peak loading, not sharp drops as with the Fyfe specimens (Figure 4-3). Such deflection behavior might possibly suggest a failure in the bond instead of timber. This observation corresponds well with the visual inspection of the Aquawrap ${ }^{\circledR}$ bond wraps. An exception being AQ6-3, which displayed the same sudden drop in capacity as on the Fyfe materials.

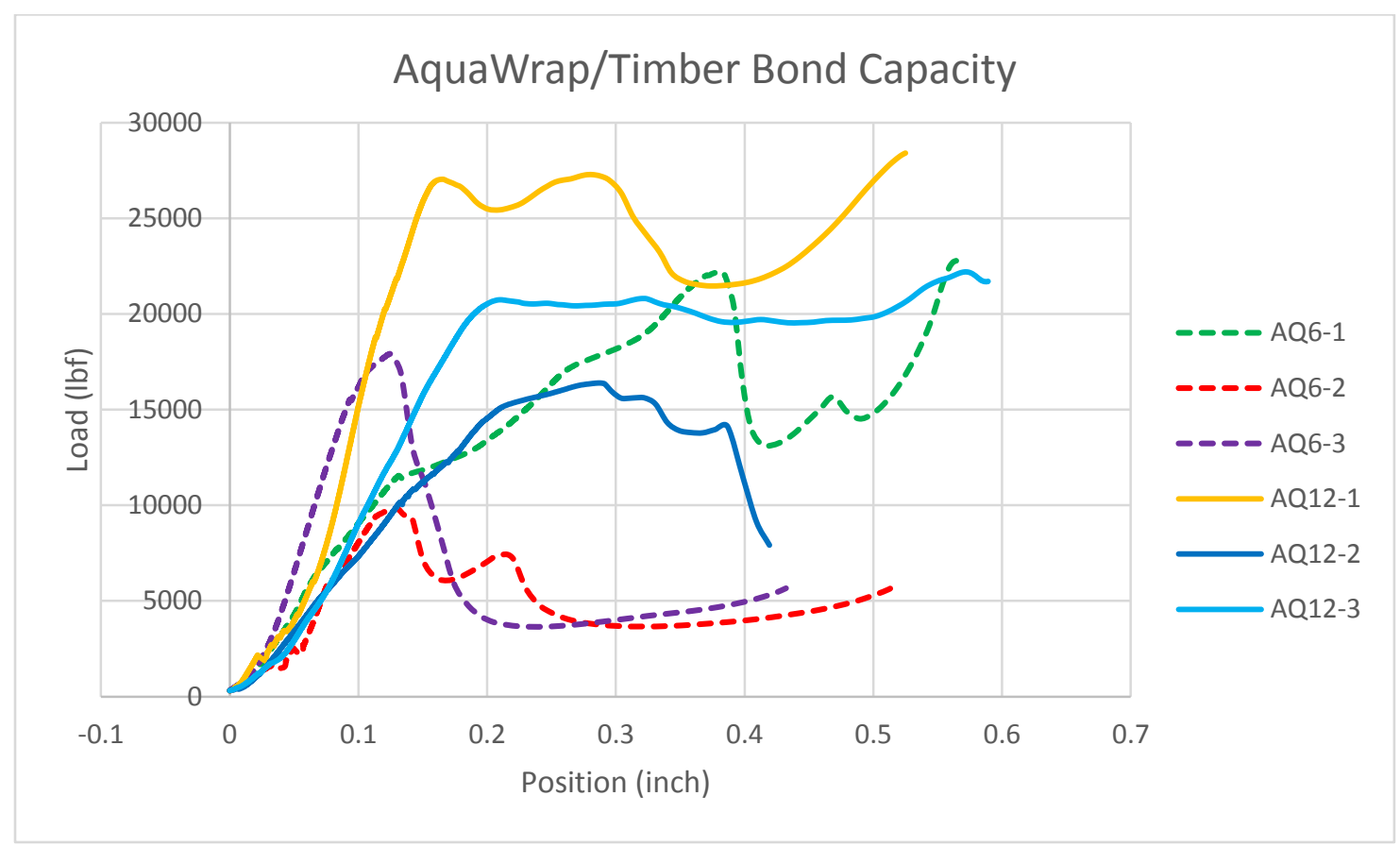

Figure 4-6: Aquawrap ${ }^{\circledR} /$ timber bond capacity (lbf)

\subsubsection{Phenolic Bond Strengths}

\subsubsection{Capacity and strength of phenolic/timber bonds}

Phenolic/timber bond specimens failed in bond slippage. Average bond strengths for 6" and 12" of lengths of bond were 112 psi and 64 psi respectively. 6 " bond lengths provided an average capacity of approximately 24.1 kips while 12 " bond lengths carried around 26 kips. Results from the bond evaluations are reported for the 6" and 12" phenolic bond lengths are shown 
in Table 4-5 and Table 4-6. Due to the limited increase in capacity from 6" to 12" bond lengths (only 7\%), it is likely that the phenolic material has an optimum bond length between 6" and 12". Increasing past 12 " of bond length will not provide much more additional capacity.

Table 4-5: Phenolic 6" Bond Lengths

\begin{tabular}{|c|c|c|c|c|}
\hline Sample & ID & $\begin{array}{l}\text { Bond } \\
\text { Area } \\
\left(\mathrm{in}^{\wedge} 2\right) \\
\end{array}$ & $\begin{array}{c}\text { Peak } \\
\text { Load } \\
\text { (lbf) }\end{array}$ & $\begin{array}{c}\text { Peak } \\
\text { Stress } \\
(\mathrm{psi})\end{array}$ \\
\hline PH6-1 ${ }^{1}$ & 6P3-3 & 206 & 19,279 & 93 \\
\hline PH6-21 & 6P1-2 & 221 & 28,518 & 129 \\
\hline PH6-31 & $6 \mathrm{P} 1-3$ & 216 & 24,523 & 114 \\
\hline \multirow{2}{*}{\multicolumn{3}{|c|}{$\begin{array}{r}\text { Average: } \\
\text { COV: }\end{array}$}} & 24,107 & 112 \\
\hline & & & $19 \%$ & $16 \%$ \\
\hline
\end{tabular}

Table 4-6: Phenolic 12" Bond Lengths

\begin{tabular}{|c|c|c|c|c|}
\hline Sample & ID & $\begin{array}{l}\text { Bond } \\
\text { Area } \\
\left(\text { in`2 }^{\wedge}\right)\end{array}$ & $\begin{array}{c}\text { Peak } \\
\text { Load } \\
\text { (lbf) }\end{array}$ & $\begin{array}{c}\text { Peak } \\
\text { Stress } \\
(\mathrm{psi})\end{array}$ \\
\hline PH12-1 ${ }^{1}$ & $12 \mathrm{P} 1-2$ & 405 & 30,754 & 76 \\
\hline $\mathrm{PH} 12-2^{12}$ & $12 \mathrm{P} 2-3$ & 381 & 23,756 & 62 \\
\hline PH12-3 $3^{12}$ & $12 \mathrm{P} 3-1$ & 432 & 23,481 & 54 \\
\hline \multirow{2}{*}{\multicolumn{3}{|c|}{$\begin{array}{r}\text { Average: } \\
\text { COV: }\end{array}$}} & 25,997 & 64 \\
\hline & & & $16 \%$ & $17 \%$ \\
\hline
\end{tabular}

${ }^{1}$ bond slippage $/{ }^{2}$ eccentricity present

\subsubsection{Visual inspection of phenolic/Timber bonds after testing}

Cutting off the phenolic wrap after bond testing revealed that a thin layer of timber remained on the entire surface of the wrap as shown in Figure 4-7 and Figure 4-8. The retained timber seemed to indicate failure in the timber and not in the bond. The retained timber layer was superficial (>1/32"). This may indicate that the outer layer of timber was weakened to creosote treatment and degraded further from the heat of the chemical reactions. 


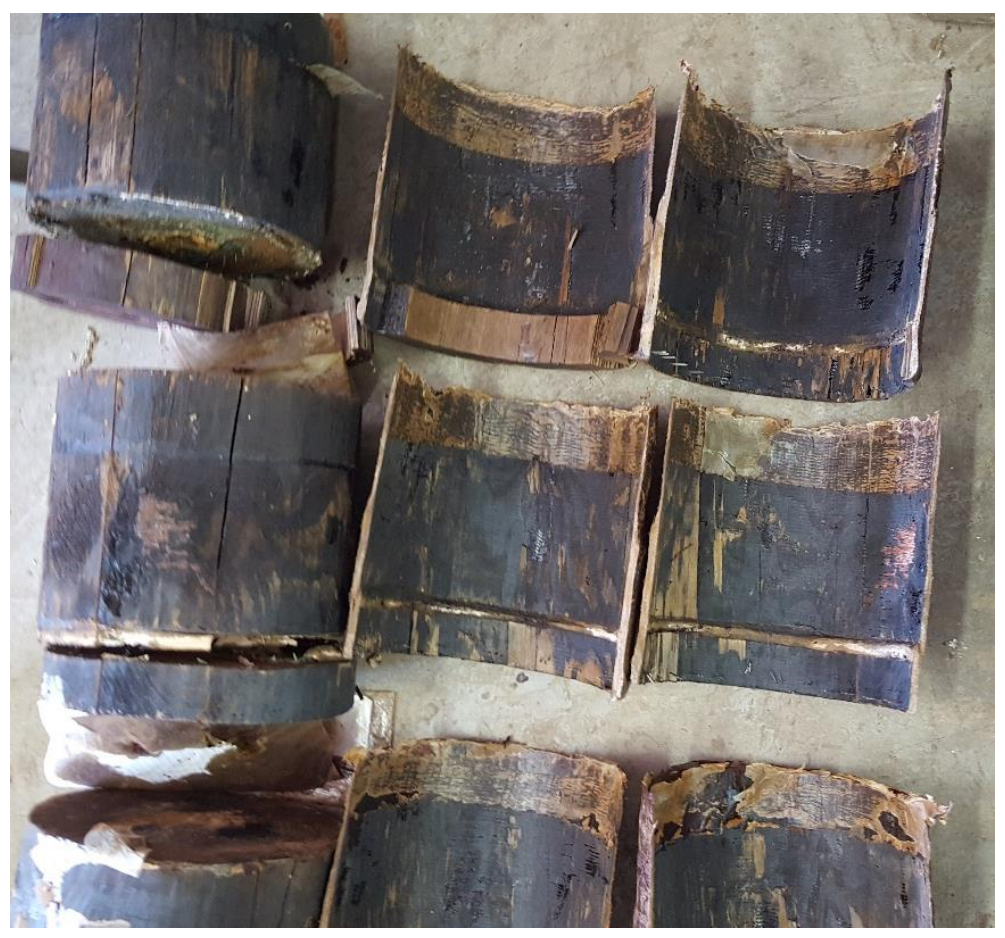

Figure 4-7: Superficial retained timber on 6" Phenolic wraps

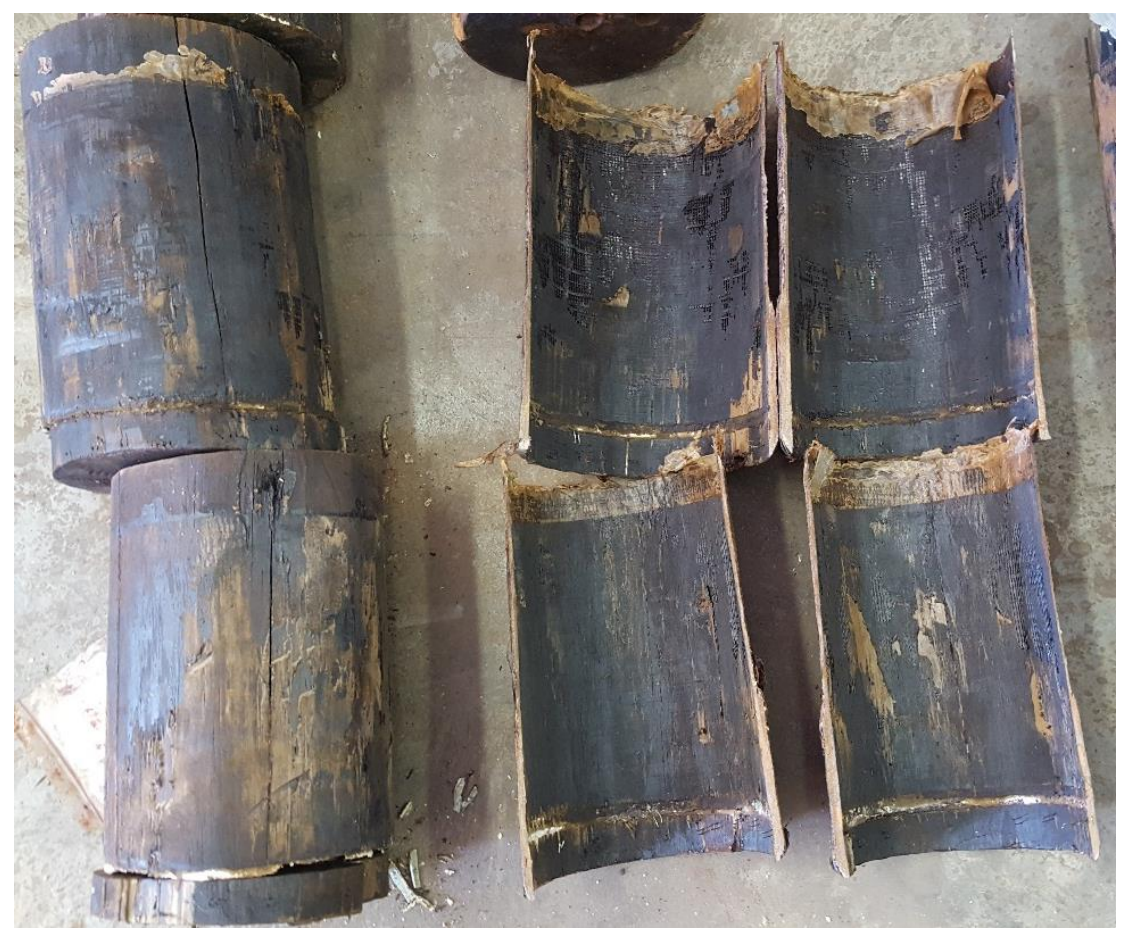

Figure 4-8: Superficial retained timber on 12" phenolic wraps 


\subsubsection{Plot of load vs deflection of phenolic pushout}

Similar behavior, as to the Fyfe bond specimens, was present in the phenolic samples as shown in Figure 4-9, potentially indicating timber failure. Visual inspections also seemed to verify this observation.

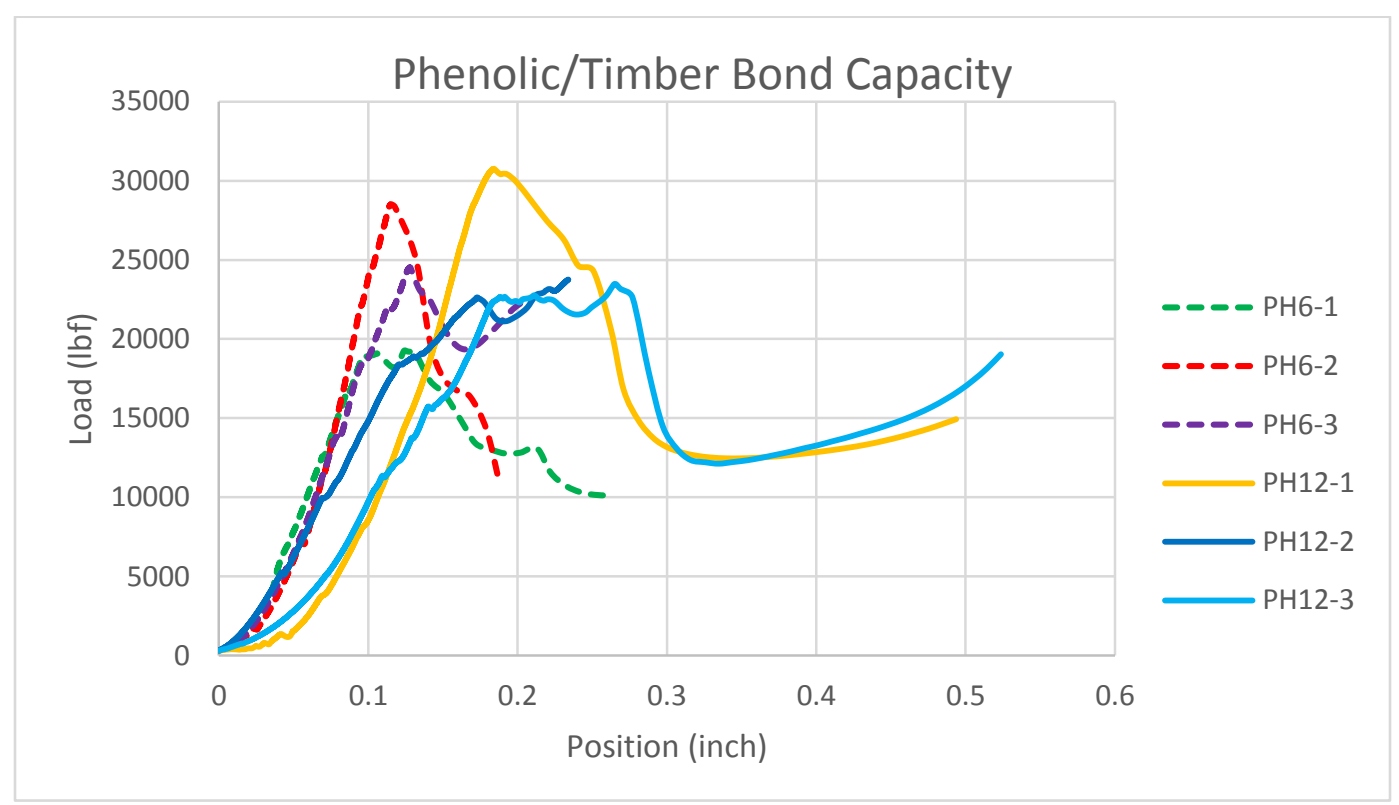

Figure 4-9: Phenolic/timber bond capacity (lbf)

\subsubsection{Discussion of push-out bond evaluations}

The discussion of the push-out results includes, discussion of failure modes, and possible influences on the bond performance including primers, types of fabric used in the wraps, eccentric loading, variations within timbers, and summary. Finally, the results are compared with typical design criteria for timber piles in the field.

\subsubsection{Comparison of results}

The bond strengths and capacities for the Fyfe, Aquawrap®, and phenolic systems evaluated are included in Table 4-7. Overall increasing the bond area increased the overall capacity of the bond, but the bond strengths were always reduced. Such behavior suggests a non-linear 
relationship between wrap length and bond strength. More testing would be needed to clearly establish the bond length-strength relationship. The Fyfe wraps provided the highest bond strengths and capacities by far. The Aquawrap ${ }^{\circledR}$ systems displayed low average bond strengths and capacities. For the phenolic wraps, the bond strengths were low, but it was also interesting to note that increasing the bond length did not significantly increase the bond capacity $\approx 7 \%$ increase). (Such behavior suggests that an optimum bond length for the phenolic system exists between 6" and 12"; increasing bonds past this will not necessarily provide more axial capacity). Possible explanations for these results are discussed below.

Table 4-7: Summary of strengths and capacity by bond length

\begin{tabular}{|l|r|r|c|c|}
\hline \multirow{2}{*}{ System } & \multicolumn{2}{|c|}{ Strength (psi) } & \multicolumn{2}{c|}{ Capacity (lbf) } \\
\cline { 2 - 5 } & \multicolumn{1}{c|}{$6 "$} & \multicolumn{1}{c|}{$12^{\prime \prime}$} & \multicolumn{1}{c|}{6} & \multicolumn{1}{c|}{$12^{\prime \prime}$} \\
\hline Fyfe & 316 & 240 & 61,150 & 90,541 \\
\hline Aquawrap® & 99 & 56 & 20,352 & 22,336 \\
\hline Phenolic & 112 & 64 & 24,107 & 25,997 \\
\hline
\end{tabular}

\subsubsection{Failure modes of the FRP/timber bond types}

Inspection of specimens after evaluations showed that both the Fyfe and phenolic systems failed predominately in the timber on the delaminated wrap. Aquawrap ${ }^{\circledR}$ specimens failed consistently in the bond with no retained timber. The plots for 6" (Figure 4-10) and 12" (Figure 4-11) bond behavior seem to correspond well to the assumption that timber failures display sudden drops in capacity with a rapid increase in deformation while bond failures display longer sustained loads before deflection. 


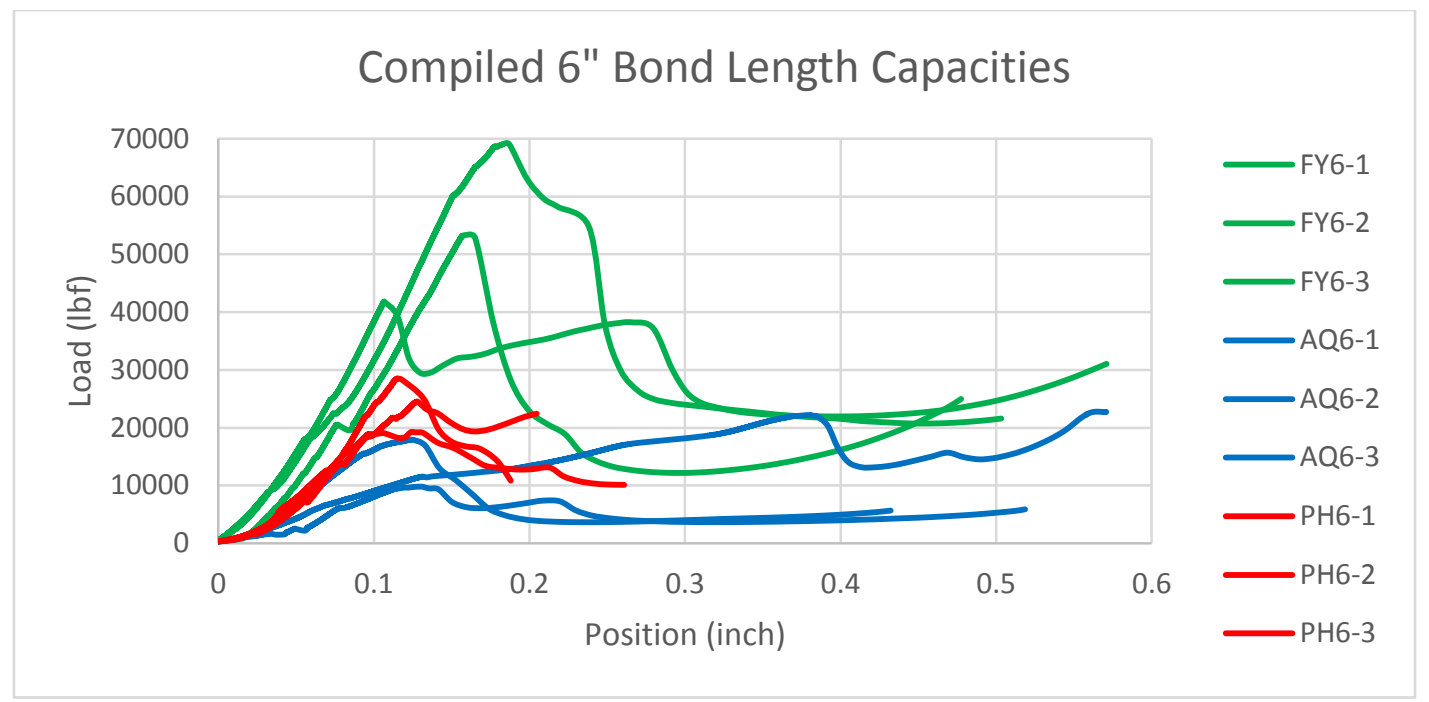

Figure 4-10: Compiled 6" bond lengths capacities

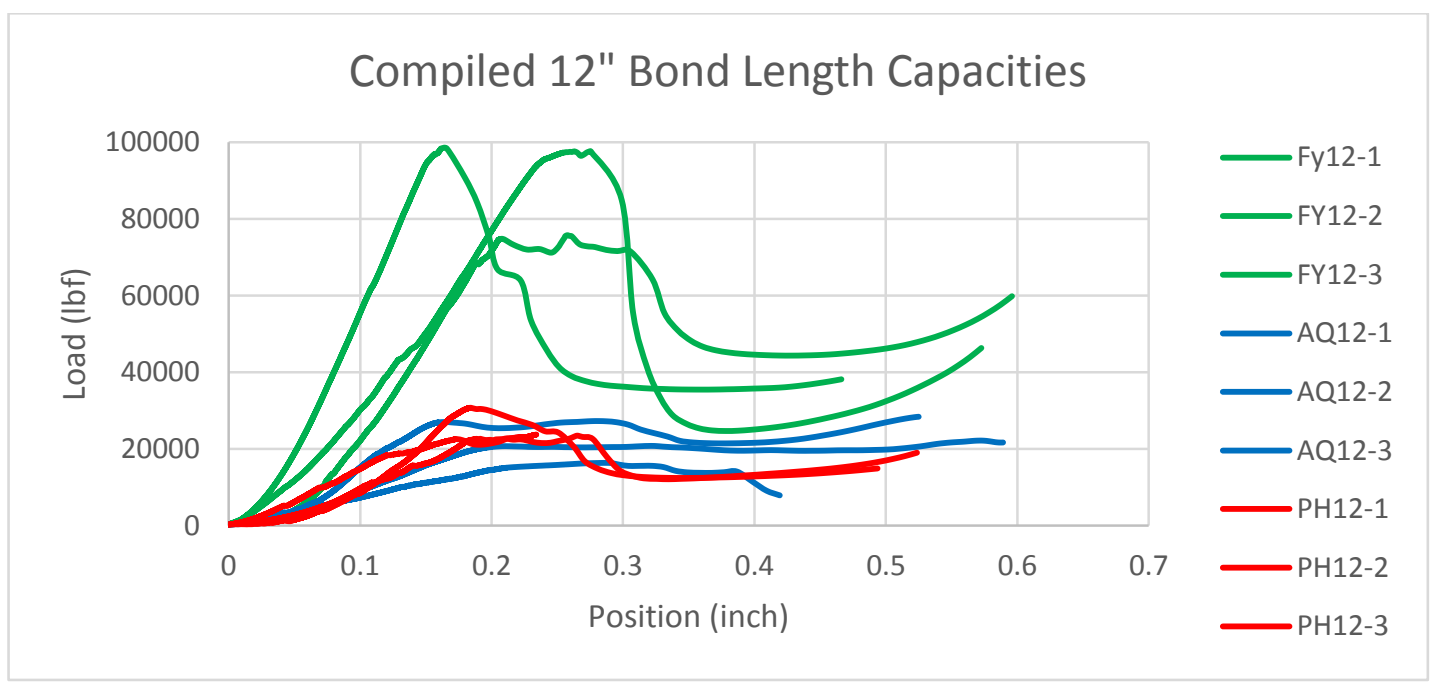

Figure 4-11: Compiled 12" bond lengths capacities

\subsubsection{Influences from primers/resins}

From the literature review, good bond between timber and wrap is a function of chemically compatible primer/resin systems with glass.

For the Fyfe system, the epoxy resin was used as both a primer (low viscosity) and a saturating resin for the fabric. It is possible that the low viscosity $(600-700 \mathrm{cps})$ and low set time (6 hour) of the resin allowed for deeper penetration of epoxy into timber during the curing process. 
Deeper resin penetration could account for the higher bond strengths and larger portions of retained timber (1/4" to $1 / 2 ")$ on the wrap surface after failure.

Phenolic wraps also used the saturating resin as a primer. However the phenolic resin had a higher viscosity (700-800 cps) and a much faster curing time (1 hour) than the epoxy used in the Fyfe system which might indicate that the phenolic resin did not penetrate the wood as deeply. A shallower penetration could be responsible for the lower bond strengths and superficial layers $(1 / 32 ")$ of retained timber on the wrap surface.

Aquawrap® did not use a primer system for wrapping. Instead, the timber and the wrap were sprayed with water to activate the curing process and then directly installed on the timber with the assumption that the resin impregnated in the fabric would bond with the timber. Using this manufacturer recommended application process, the wraps did not seem to develop deep penetrating timber bonds. Three explanations are possible. First, the use of water instead of a resin primer did not properly prepare the creosote treated wood for bonding. The water likely filled the wood pores and could not be absorbed further into the creosote treated timber. With water filled pores, it is speculated that the resin could not penetrate any deeper into the wood, creating surface

level bonds. Second, the polyurethane resin cured quickly once activated (30 minutes), which likely made deep timber penetration difficult, creating a low strength bonds as with the phenolic system. Third, the preimpreganated wraps ensured a consistent fiber volume fraction in the system, but also restricted resin from flowing freely into the timber, which could have further inhibited resin penetration into timber pores from outer surfaces.

\subsubsection{Influences from fabric stiffness}

During wrapping, it was noted that the stiffness of the fabric utilized for wrapping influenced how well the wraps conformed to the perimeter of the timber samples. 
The Fyfe and phenolic systems both utilized wet layup techniques that permitted the fabric to remaining flexible during installation, enabling the wrap to conform to flat surfaces and other irregularities with full contact. Few exceptions to full contact existed except for a few specimens, which displayed slight debonding near the tops of the piles due in part to tapered sections (changing diameter along the length of the pile).

Aquawrap® fabrics were much stiffer due to the preimpreganated nature of the system. This stiffness made it difficult for the wrap to maintain good (if not 100\%) contact around the pile especially on flat surfaces. The Aquawrap® tended to bridge over flat spots on the pile (Figure 4-12) and would not conform to areas were the pile tapered (Figure 4-13). The lack of complete bonding could account for the lower bond performance of the Aquawrap® wraps.

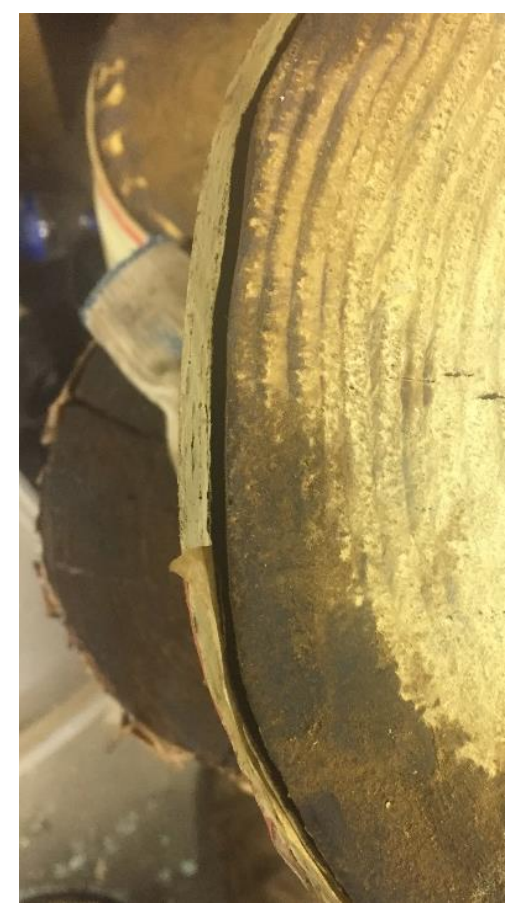

Figure 4-12: Lack of bond around flat edge of AQ6-3 (6P2-4) 


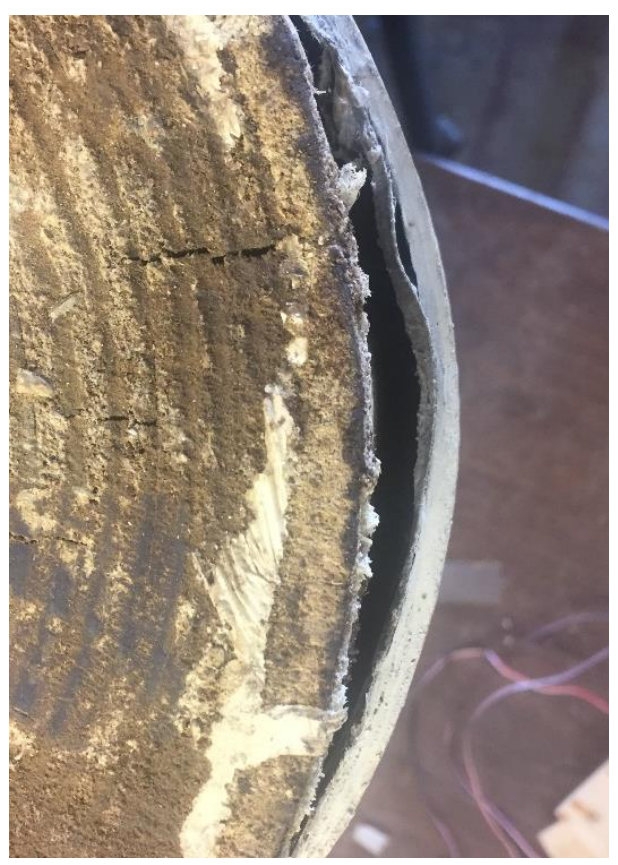

Figure 4-13: De-bond on rounded Aquawrap ${ }^{\circledR}$ bond specimen AQ12-2 (12P2-2)

\subsubsection{Influences of eccentricities}

As discussed previously in Section 3.2.2, the timber specimens that were wrapped did not possess perfectly parallel testing surfaces due to the variations inherent when cutting with a handheld chainsaw. The cutting process also at times left a slightly uneven top or base (slopes, high spots, waves, etc.) which might have not evenly distributed the load in the specimens. While the adjustable testing head rotated to adjust for smooth, non-parallel surfaces, these surface defects possibly created eccentric conditions with uneven load distributions.

The most noticeable eccentricities were in the 12 " bond length phenolic samples. To correct this eccentricity on specimen PH12-1, an elastomeric pad was placed under an edge to evenly distribute the load throughout the specimen as shown in Figure 4-14. The pad did provide a $24 \%$ increase in bond strength compared to the other specimens evaluated (see Table 4-6), but the pad was not used on the other 12 " phenolic bond length samples or any other specimens. The exact influences of the eccentricities are not recorded for all of the specimens, but lack of 
elastomeric pad is still expected to have produce some capacity decrease on some samples. However, unanticipated eccentric loads are likely to be found in field conditions, thus the results obtained herein are applicable to real world conditions.

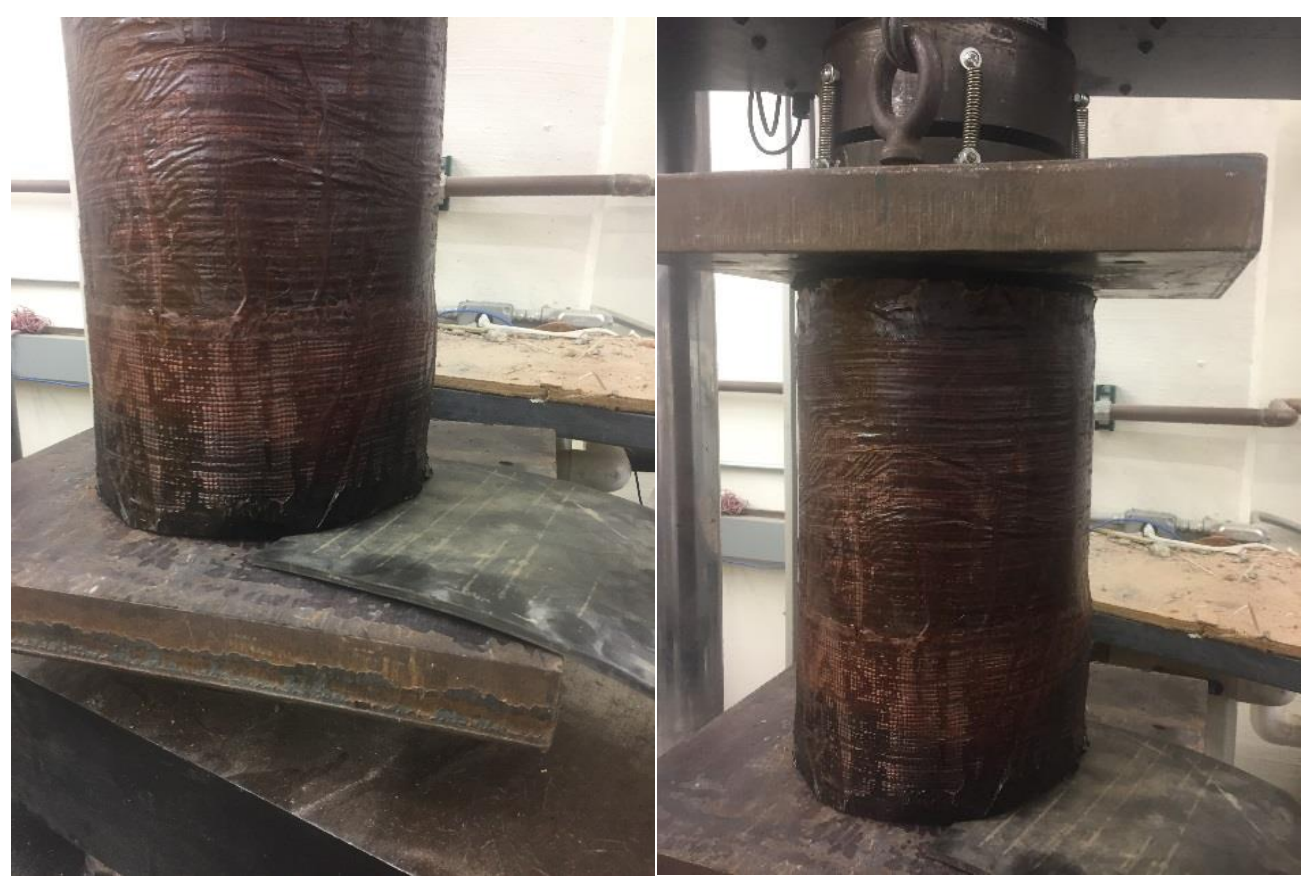

Figure 4-14: Phenolic bond specimen with pad to negate eccentricity (12p2-1)

\subsubsection{Influence of compressive capacity}

During the evaluations of the Aquawrap® bond specimens (which were tested before Fyfe and phenolic systems), all 12" bond specimens experienced composite compressive failure in the base and around the gap as shown in Figure 4-15. The failure of the base occurred because the bond strengths exceeded the compressive strengths of the wraps as presented in Section 4.4.3. Such a failure was due to the large stress concentration that developed from the square edges used to create the gap for testing.. These issues were corrected for the remaining of the bond specimens (Fyfe and phenolic systems) with the application of additional layers of FRP wrap around the base and the gap. Due to this, no further compression failures occurred during bond testing, because of circumferential reinforcing of wraps at the end. The reinforcement need at the gap and bottom is a 
test related issue and does not have any direct correlation to field applications as field applications would likely not have such an abrupt change from the bond area to the FRP carrying the load.

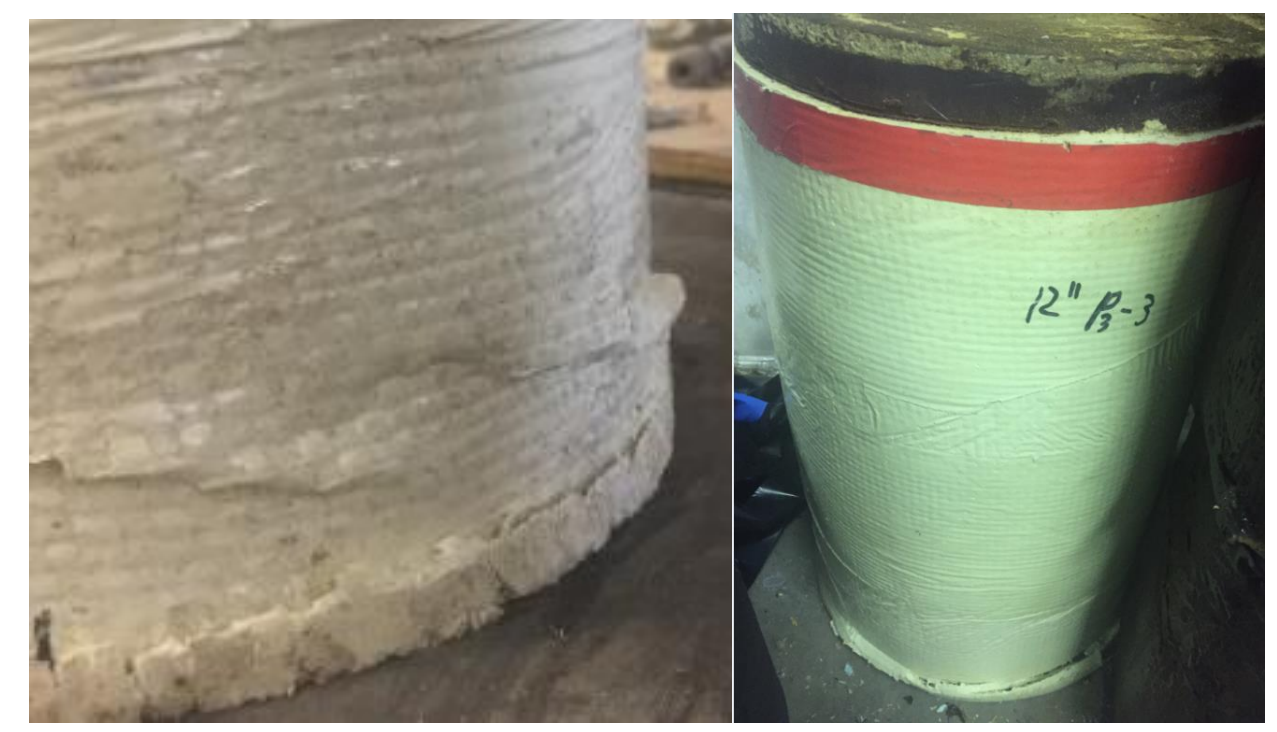

Figure 4-15: Failure from fiber crushing on AQ 12-1 (12P1-1) and AQ 12-3 (12P3-3)

\subsubsection{Influence of timber pile variability}

Timber substrates from the same pile would hypothetically display similar properties and behaviors. To avoid bonding entire systems to the same pile, samples were divided among different piles before wrapping. The influence from the pile substrates were tracked by determining if particular piles displayed higher capacities than others. It was found that samples taken from Pile ID 12P1 were consistently high while those from Pile ID 12P3 were of the lowest performances. However, no single system appeared to be influenced by the timber substrate more than the others. It is also possible that these variations were related to the other influences previously mentioned.

\subsubsection{Summary of Influences}

The various factors that negatively influenced bond failures are summarized below in Table 4-8. While many of the factors are related to the properties of the systems, a knock down factor for eccentricity should be provided in future design work as it will influence in the field. A 
recommended reduction will be provided later. One factor that will be critical for design purposes is moisture cycling. While this portion of the study did not evaluate aging, determining the long term behavior of timber/FRP bonds is critical for establishing design knock down factors in the future.

Table 4-8: Factors influencing lowered bond capacity

\begin{tabular}{|c|l|l|}
\hline Factor & \multicolumn{1}{|c|}{ Samples } & \multicolumn{1}{c|}{ Influence } \\
\hline Poor Timber Penetration/No Primer & Aquawrap ${ }^{\circledR}($ All) & Limited timber failure \\
\hline Fabric Stiffness & Aquawrap ${ }^{\circledR}($ All $)$ & Lack of full contact bond \\
\hline Compressive Failure & Aquawrap ${ }^{\circledR}\left(12^{\prime \prime}\right)$ & No bond failure \\
\hline Eccentric Load & Phenolic $\left(12^{\prime \prime}\right)$ & Reductions of $24 \%$ bond strengths \\
\hline
\end{tabular}

\subsubsection{Comparisons with timber pile capacities}

The bond evaluations were ultimately conducted to determine if repaired piles in the field could develop the equivalent capacity to a new timber pile. For a 10" diameter pile, a design capacity is recommended to be between 94 to 98 kips based on Table 3-2 of the Timber Pile Design and Construction Manual [29]. The Fyfe bond strength for 12" bond lengths most closely approached this range with an average capacity of 90.5 kips on a 10 " pile, albeit with no factor of safety. Methods to improve the bond capacity of Aquawrap ${ }^{\circledR}$ and phenolic systems should be determined before applying them to piles utilizing the full capacity. It could be possible to utilize these two systems on piles with lower services loads (18-20 kips), but further evaluations are needed for verification.

Furthermore these bond evaluations were conducted on new timbers with relatively "good" substrates which improved bond strengths. In the field, most rehabilitated piles will most likely have poorer quality substrates which would further reduce the bond strength (since Fyfe and phenolic failed in the timber, lower timber strength would result in low bond strength). This is a postulation and such contingency needs to be accounted for in design through safety factors. 
Extrapolating the bond strength losses to older timber piles in the field would require the use of knock down factors to account for aging and deterioration. As a separate study, aging evaluations are scheduled to be conducted on wrapped piles to attempt to determine design knock down factors.

\subsection{Pull off Bond Testing (Modified ASTM D7522)}

Bond pull off testing was conducted on all four systems with five pull-offs each to determine baseline pull off strength values on good timber. Results are presented for Fyfe (4.3.1), Sika (4.3.2), Aquawrap® (4.3.3), and Phenolic (4.3.4) wrap systems. Pull off tests conducted on timber piles used in the study are shown in 4.3.5. Discussion of the results is given in 4.3.6.

\subsubsection{Pull Off Bond Strength of Fyfe}

Fyfe bond pull off specimens all failed in the timber with large sections of retained timber as shown in Figure 4-16, thus showing that the FRP to timber bond in tension exceeds that of the underlying wood. Average pull off strength was 353 psi as shown in Table 4-9. The wood fibers on this particular sample displayed a large amount of variability (knots, grain changes) which contributed to the large variation between pull off tensile strengths.

Table 4-9: Fyfe Pull-off Bond Capacity and Strengths

\begin{tabular}{|c|r|c|l|}
\hline Sample & $\begin{array}{c}\text { Force } \\
\text { (lbf) }\end{array}$ & $\begin{array}{c}\text { Stress } \\
(\mathbf{p s i})\end{array}$ & Failure Mode \\
\hline FY 1 & 1133 & 360.65 & Timber \\
\hline FY 2 & 660 & 210.08 & Timber \\
\hline FY 3 & 1471 & 468.23 & Timber \\
\hline FY 4 & 1682 & 535.40 & Timber \\
\hline FY 5 & 596 & 189.71 & Timber \\
\hline AVG & 1108.4 & $\mathbf{3 5 2 . 8 1}$ & $39 \%($ COV $)$ \\
\hline \multicolumn{5}{|c}{${ }^{1}$ Failed on knot } \\
\hline
\end{tabular}



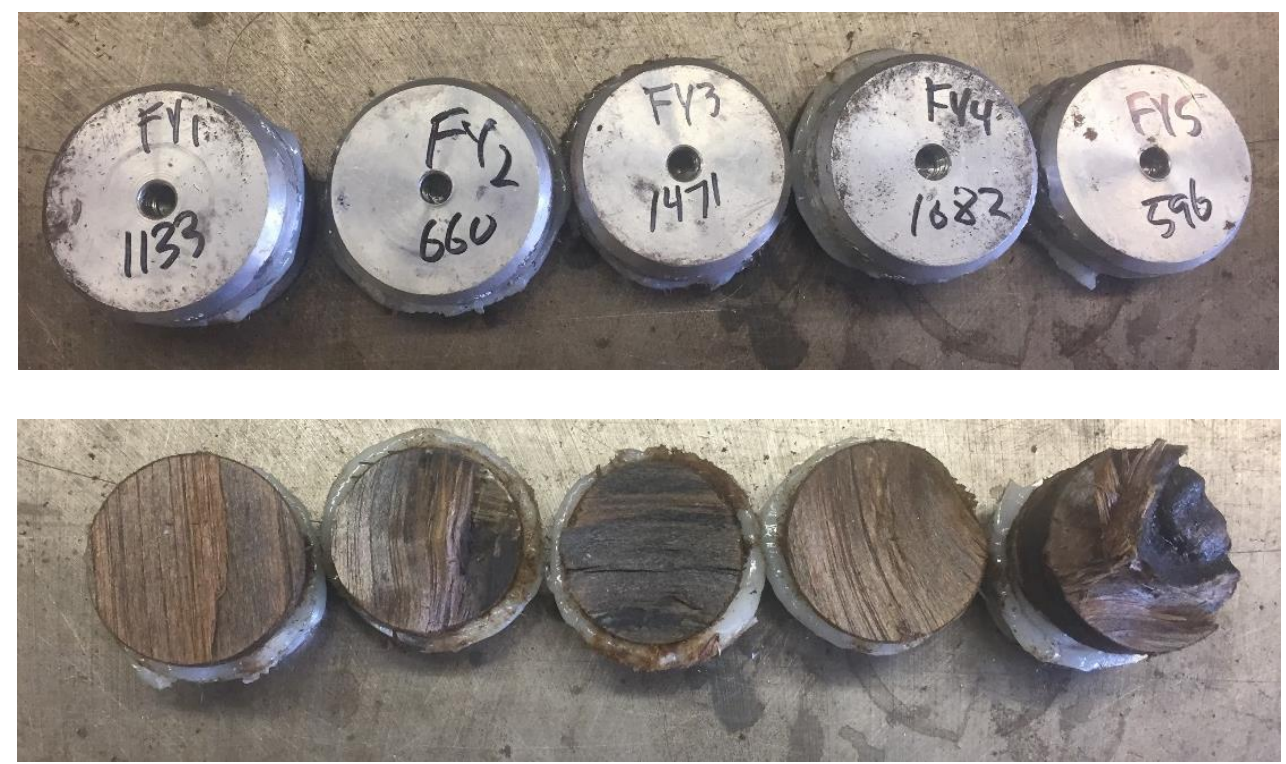

Figure 4-16: Fyfe Pull-off Carriers (Timber)

\subsubsection{Pull Off Bond Strength of Sika}

Similar to the Fyfe bond pull off specimens, Sika pull off samples all failed in the timber with large sections of retained timber as shown in Figure 4-17. Again the strength of the FRP to timber bond in tension exceeded that of the underlying wood. Excluding the extremely low value of SK2 (whose failure was caused by a crack in the timber), the average pull off strength was 316 psi as shown in Table 4-10.

Table 4-10: Sika Pull-off Bond Capacity and Strengths

\begin{tabular}{|c|r|r|l|}
\hline Sample & $\begin{array}{c}\text { Force } \\
\text { (lbf) }\end{array}$ & $\begin{array}{c}\text { Stress } \\
(\mathbf{p s i})\end{array}$ & Failure Type \\
\hline SK 1 & 934 & 297.30 & Timber \\
\hline SK 2 & 350 & 111.41 & Cracked Timber \\
\hline SK 3 & 1016 & 323.40 & Timber \\
\hline SK 4 & 1033 & 328.81 & Timber \\
\hline AVG & 994.333 & $\mathbf{3 1 6 . 5 1}$ & $4 \%($ COV $)$ \\
\hline
\end{tabular}

${ }^{1}$ neglected this outliner for averaging 

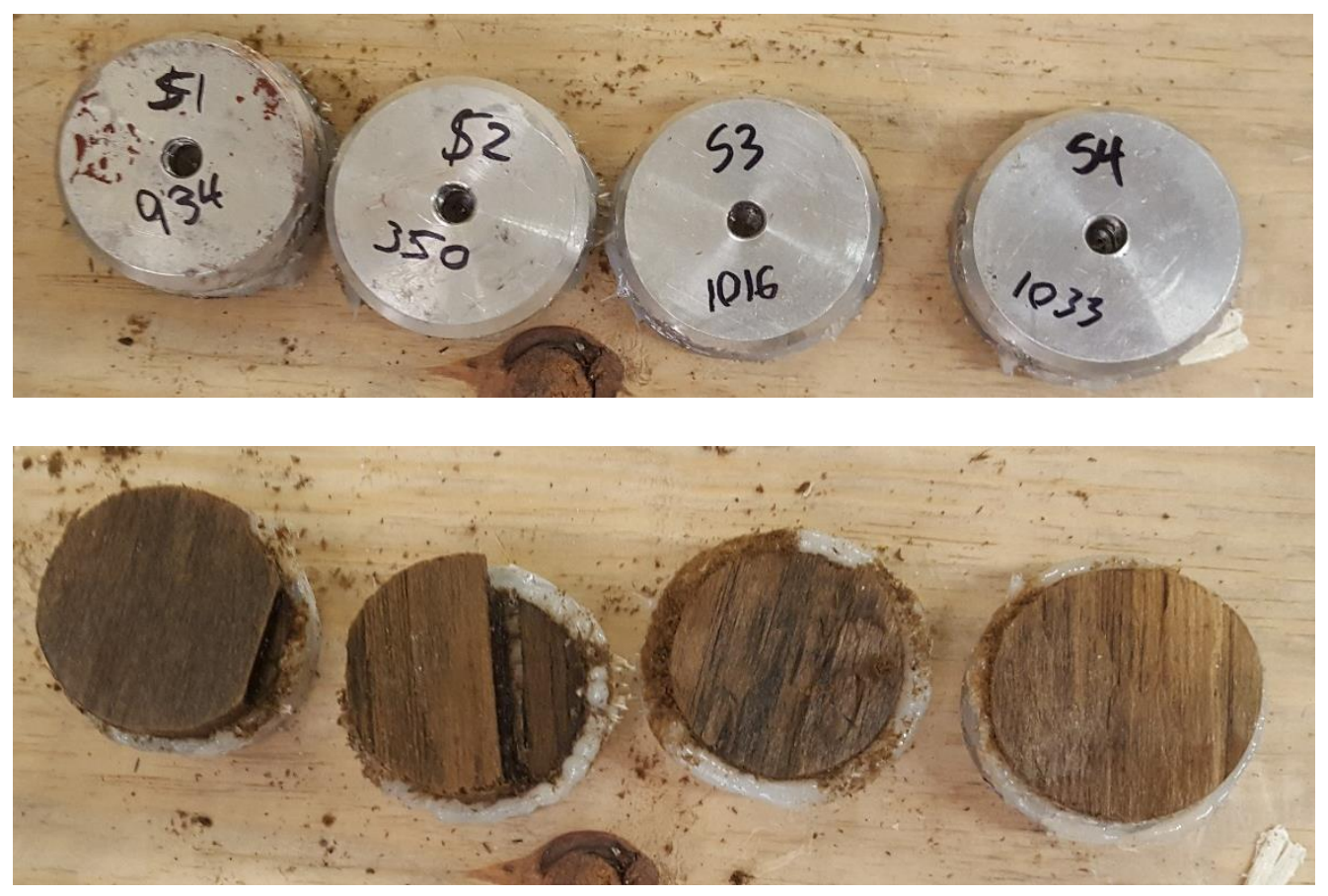

Figure 4-17: Sika Pull-off Carriers (Timber)

\subsubsection{Pull Off Bond Strength of Aquawrap ${ }^{\circledR}$}

The pull off tests for the Aquawrap® displayed failure in the bond line indicating poor resin penetration into the wood substrate (Figure 4-18). One of the pull off carriers had a buildup of epoxy from the carrier attachment process which produced an artificially high pull off strength. The data point was neglected along with AQ3 which carried barely any load (>20 lbf). Average pull off strength was 34 psi as shown in Table 4-11.

Table 4-11: Aquawrap ${ }^{\circledR}$ Pull-off Bond Capacity and Strengths

\begin{tabular}{|c|r|r|l|}
\hline Sample & $\begin{array}{c}\text { Force } \\
\text { (lbf) }\end{array}$ & $\begin{array}{c}\text { Stress } \\
(\mathbf{p s i})\end{array}$ & Failure Type \\
\hline AQ 1 & 141 & 44.88 & Bond line \\
\hline AQ 2 & 99 & 31.51 & Bond line \\
\hline AQ 3 & 18 & 5.73 & Bond line \\
\hline AQ 5 & 105 & 33.42 & Bond line \\
\hline AQ 6 & 82 & 26.10 & Bond line \\
\hline AVG & 106.75 & $\mathbf{3 3 . 9 8}$ & 20\% (COV) \\
\hline
\end{tabular}

${ }^{1}$ Excluded from average 

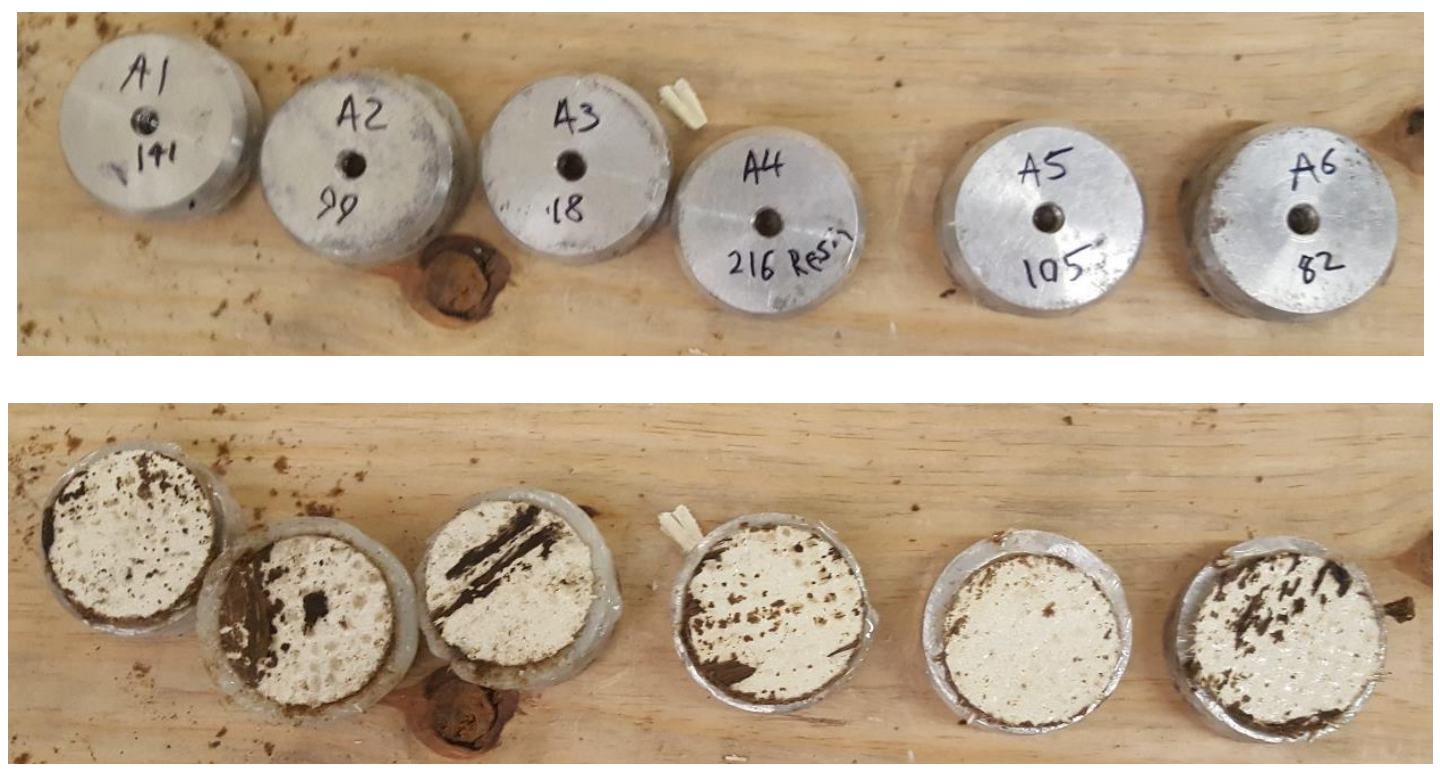

Figure 4-18: Aquawrap ${ }^{\circledR}$ Pull-off Carriers (Bond line)

\subsubsection{Pull Off Bond Strength of Phenolic}

Phenolic pull off specimens exhibited timber failures, shown in Figure 4-19, with less retained timber than the Fyfe pull off samples. However, it again showed that the FRP to timber bond in tension was greater than that of underlying timber. Average pull off strength was $173 \mathrm{psi}$ as shown in Table 4-12.

Table 4-12: Phenolic Pull-off Bond Capacity and Strengths

\begin{tabular}{|c|c|c|l|}
\hline Sample & $\begin{array}{c}\text { Force } \\
\text { (lbf) }\end{array}$ & $\begin{array}{c}\text { Stress } \\
(\mathbf{p s i})\end{array}$ & Failure Type \\
\hline PH 1 & 514 & 163.61 & Timber \\
\hline PH 2 & 549 & 174.75 & Timber \\
\hline PH 3 & 426 & 135.60 & Timber \\
\hline PH 4 & 613 & 195.12 & Timber \\
\hline PH 5 & 613 & 195.12 & Timber \\
\hline AVG & 543 & $\mathbf{1 7 2 . 8 4}$ & $13 \%(C O V)$ \\
\hline
\end{tabular}



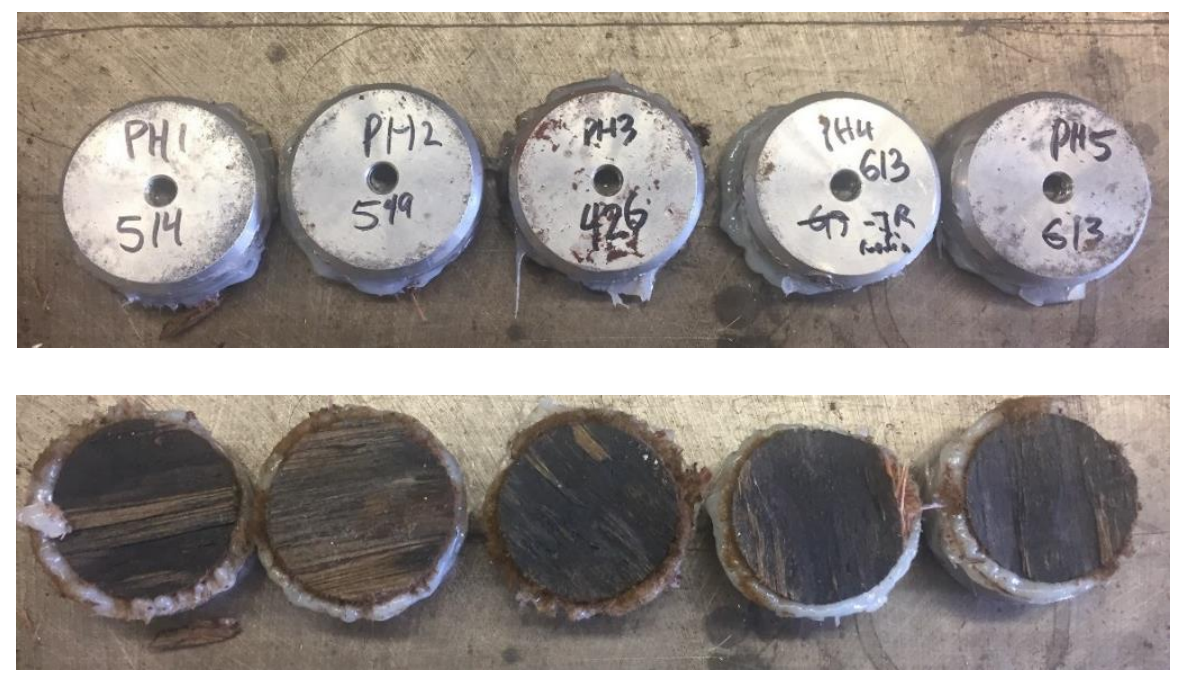

Figure 4-19: Phenolic Pull-off Pucks (Timber)

\subsubsection{Pull Off Capacity of Timber}

Pull off testing was completed on various locations of creosote treated timber piles and the results are included in Table 4-13. The piles tested were taken from specimens previously tested for push out bond strengths (Aquawrap®). Resin and outer layers of creosote were sanded off before attaching pull off pucks. Average capacity of the timber under pull off was 235 psi, but varied from 130 psi to 383 psi.

Table 4-13: Pull off Bond Capacity

\begin{tabular}{|c|r|r|l|}
\hline Sample & \multicolumn{1}{|c|}{$\begin{array}{c}\text { Load } \\
\text { (lbf) }\end{array}$} & $\begin{array}{c}\text { Stress } \\
\text { (psi) }\end{array}$ & Failure Type \\
\hline P1C1 & 508 & 162 & Timber \\
\hline P1C2 ${ }^{1}$ & 666 & 212 & Timber surface \\
\hline P1C3 ${ }^{1}$ & 543 & 173 & Bondline \\
\hline P1T4 1 & 753 & 240 & Timber surface \\
\hline P1C5 & 689 & 219 & Timber \\
\hline P2C1 & 1203 & 383 & Timber \\
\hline P2C2 & 1092 & 348 & Timber \\
\hline P2T3 & 525 & 167 & Timber \\
\hline P2C4 & 409 & 130 & Timber \\
\hline P2C5 & 981 & 312 & Timber \\
\hline AVG & 737 & $\mathbf{2 3 5}$ & $34.7 \%(C O V)$ \\
\hline
\end{tabular}

'no full bond area due to poor glue application 


\subsubsection{Discussion of pull-off results}

\subsubsection{Comparison between systems}

Results from the pull off evaluations are summarized in Table 4-14. The Fyfe and Sika systems displayed the highest pull off strengths.

Table 4-14: Summary of Pull off Strengths

\begin{tabular}{|l|r|l|}
\hline \multicolumn{1}{|c|}{ System } & \multicolumn{1}{c|}{$\begin{array}{c}\text { Strength } \\
\text { (psi) }\end{array}$} & \multicolumn{1}{|c|}{$\begin{array}{c}\text { Failure } \\
\text { Mode }\end{array}$} \\
\hline Fyfe & 353 & Timber \\
\hline Sika & 317 & Timber \\
\hline Aquawrap $®$ & 34 & Bond line \\
\hline Phenolic & 173 & Timber \\
\hline Timber & 235 & N/A \\
\hline
\end{tabular}

Note that the strengths for these systems were higher than the timber itself. Two possible explanation could exist for the higher capacity. First, the timber substrates to which the systems were bonded have higher pull off strengths than the un-bonded timbers. Second it is possible that the epoxy resin penetrated deeply into the timber, increasing the pull off strengths. More testing on different piles would be needed to further understand the behavior. Further discussions on possible influences on the bond strengths were provided in Section 4.2.5 (pushout results).

\subsubsection{Comparison with push-out bond testing results}

The two bond tests conducted in this study evaluated two different bond properties, shear and pull-off strengths. The shear evaluations (pushout tests) were conducted from a design point of view to determine the axial capacity based on bond length. Pull off tests were conducted to determine any correlations between the shear transfer in the push out tests and the tensile transfer in the pull out tests, as pull out tests are likely to be used to verify proper field installation. For 
comparison purposes, the average pushout and pull off strengths are provided for each system in Table 4-15.

Table 4-15: Comparison with pull off and average pushout bond strengths (psi)

\begin{tabular}{|l|r|r|r|}
\hline \multicolumn{1}{|c|}{ System } & \multicolumn{1}{c|}{$\begin{array}{c}\text { Pull off } \\
\text { (psi) }\end{array}$} & $\begin{array}{c}\text { Avg } \\
\text { Pushout } \\
\text { (psi) }\end{array}$ & $\begin{array}{c}\text { Factor } \\
\text { Difference }\end{array}$ \\
\hline Fyfe & 353 & 278 & 1.36 \\
\hline Aquawrap® & 34 & 78 & 0.44 \\
\hline Phenolic & 173 & 88 & 1.97 \\
\hline
\end{tabular}

As seen, the Fyfe system displayed the highest strengths in both evaluations. Both Fyfe and phenolic systems displayed timber failures in both bond tests. Aquawrap® displayed the lowest bond strengths for both evaluations with limited timber retention on the wraps.

\subsubsection{Comparison of field installed phenolic system with phenolic system}

Phenolic wraps were installed by WVU-CFC on deteriorated piles on bridges on South Branch Valley Railroad (SBVR) in 2010. Pull off tests were conducted on piles on bridges designated 36.7 and 35.7 using the same procedure as in the lab. Average bond strengths were found to be 37 psi and 35 psi respectively as shown by the values as recorded in Table 4-16 and Table 4-17. Determination of the failure type was difficult as the color of the resin and the creosote leaching from piles were nearly identical, but none of the samples removed timber.

Table 4-16: Bridge 36.7 pull off strengths

\begin{tabular}{|r|r|r|l|}
\hline Sample & \multicolumn{1}{|c|}{ Load } & Stress & Failure Type \\
\hline 1 & 93 & 30 & Bond \\
\hline 2 & 123 & 39 & Bond \\
\hline 4 & 134 & 43 & Partial timber/bond \\
\hline Average & 117 & $\mathbf{3 7}$ & $14.9 \%(\mathrm{COV})$ \\
\hline
\end{tabular}


Table 4-17: Bridge 35.7 pull off strengths

\begin{tabular}{|r|r|r|l|}
\hline Sample & Load & Stress & \multicolumn{1}{|c|}{ Failure Type } \\
\hline 1 & 76 & 24 & surface of wood \\
\hline 2 & 93 & 30 & surface of wood \\
\hline 3 & 93 & 30 & Partial surface/bond \\
\hline 4 & 175 & 56 & Partial surface/bond \\
\hline Average & 109.25 & $\mathbf{3 5}$ & $35.3 \%(\mathrm{COV})$ \\
\hline
\end{tabular}

Clearly the values from the field were lower than those in the laboratory by a factor of almost 5 (lab values of 173 psi vs 35 psi). Additional testing would be required to verify those capacities. Failure types for two of the field pull offs were mainly in the timber portions possibly indicating that the lowered bond strengths could be caused by the deteriorated wood and not the strength of the bond.

\subsubsection{Future testing}

To verify the controlling factor influencing the pull off capacities (whether timber substrate or strength of bond), another round of pull off testing on a variety of piles for each system is recommended.

\subsection{Compression Testing}

The results presented are the maximum load and stress for each specimen along with the load versus deflection plots. Compressive results are presented for Fyfe (4.4.1), Sika (4.4.2), Aquawrap® (4.4.3), and Phenolic (4.4.4). Specimens designated A, B, and C are specimens with three layers, and specimens label D, E, and F are specimens with five layers of wrap (Exceptions to this notation are noted when appropriate). Additionally the tensile coupon test results are given in Section 4.4.5. Discussion of the results is provided in Section 4.4.6. 


\subsubsection{Fyfe Compression Results}

\subsubsection{Compressive capacity and strength of the Fyfe wraps}

The Fyfe specimens generally failed in pure compression of the fibers after matrix failure for both three and five layers or wrap as shown in Figure 4-20. The exception to this was Fyfe A which failed in compression and bending due to eccentric loading. The maximum compressive loads and stresses of the Fyfe shells are presented in Table 4-18 for three layers wrap and Table 4-19 for five layers of wrap. The maximum average stress for three layers was $14.5 \mathrm{ksi}$ and 15.5 ksi for five layers. As noted on the tables, large wrinkles in the shells possibly influenced the performance of the systems; more in depth discussions will be provided in 4.4.6.2. Note: The fiber volume fraction was assumed to be $30 \%$ for hand layup methods

Table 4-18: Fyfe Three Layer Compression Results

\begin{tabular}{|c|c|c|c|c|}
\hline Specimen & $\begin{array}{c}\text { Area } \\
(\text { in^^2) }\end{array}$ & $\begin{array}{c}\text { Load } \\
\text { (lbf) }\end{array}$ & $\begin{array}{l}\text { Stress } \\
\text { (psi) }\end{array}$ & FVF \\
\hline Fyfe $A^{1}$ & 4.11 & 39,075 & 9,510 & $30 \%$ \\
\hline Fyfe B & 4.11 & 70,600 & 17,183 & $30 \%$ \\
\hline Fyfe $C^{2}$ & 4.17 & 49,324 & 11,838 & $30 \%$ \\
\hline \multirow{2}{*}{\multicolumn{2}{|c|}{$\begin{array}{r}\text { Average: } \\
\text { COV: }\end{array}$}} & 59,962 & 14,511 & \\
\hline & & $17.7 \%$ & $18.4 \%$ & \\
\hline
\end{tabular}

${ }^{1}$ Off-center positioning/2large wrinkle (both neglected in average)

Table 4-19: Fyfe Five Layer Compression Results

\begin{tabular}{|c|c|c|c|c|}
\hline Specimen & $\begin{array}{c}\text { Area } \\
(\text { in^^2) }\end{array}$ & $\begin{array}{c}\text { Load } \\
\text { (lbf) }\end{array}$ & $\begin{array}{c}\text { Stress } \\
(\mathbf{p s i})\end{array}$ & FVF \\
\hline Fyfe $D^{2}$ & 7.45 & 106,130 & 14,252 & $30 \%$ \\
\hline Fyfe E & 7.45 & 125,490 & 16,851 & $30 \%$ \\
\hline Fyfe $F^{2}$ & 7.45 & 96,546 & 12,965 & $30 \%$ \\
\hline \multirow{2}{*}{\multicolumn{2}{|c|}{$\begin{array}{r}\text { Average: } \\
\text { COV: }\end{array}$}} & 115,810 & 15,551 & \\
\hline & & $8.4 \%$ & $8.4 \%$ & \\
\hline
\end{tabular}




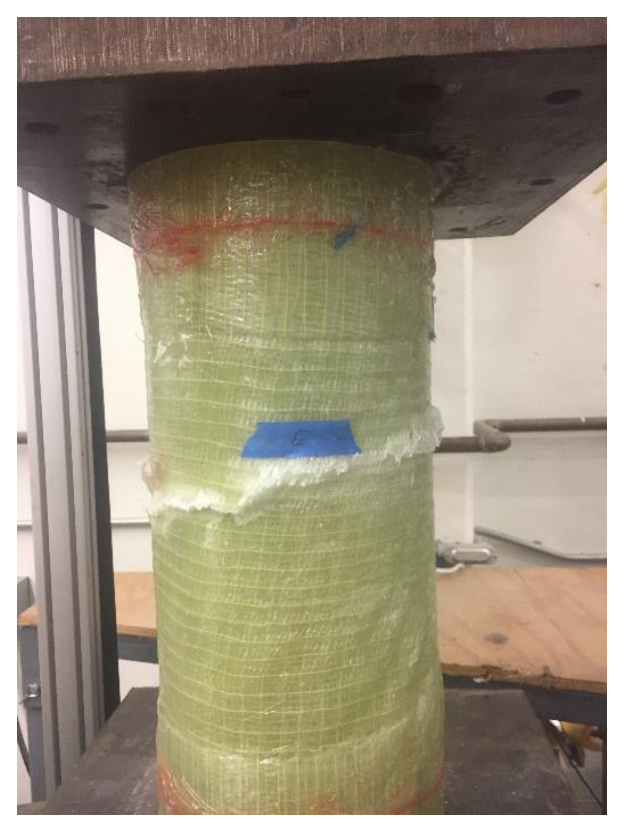

Figure 4-20: Typical Fyfe crushing compression failure (Fyfe E)

\subsubsection{Plots of Fyfe load versus deflection}

It can be seen in Figure 4-21 that the slope of the five layer samples the shells under load are relatively the same which could indicated a consistent (stiffness) modulus of elasticity of the wrap. The failures displayed abrupt drops likely due to the failure in the fibers.

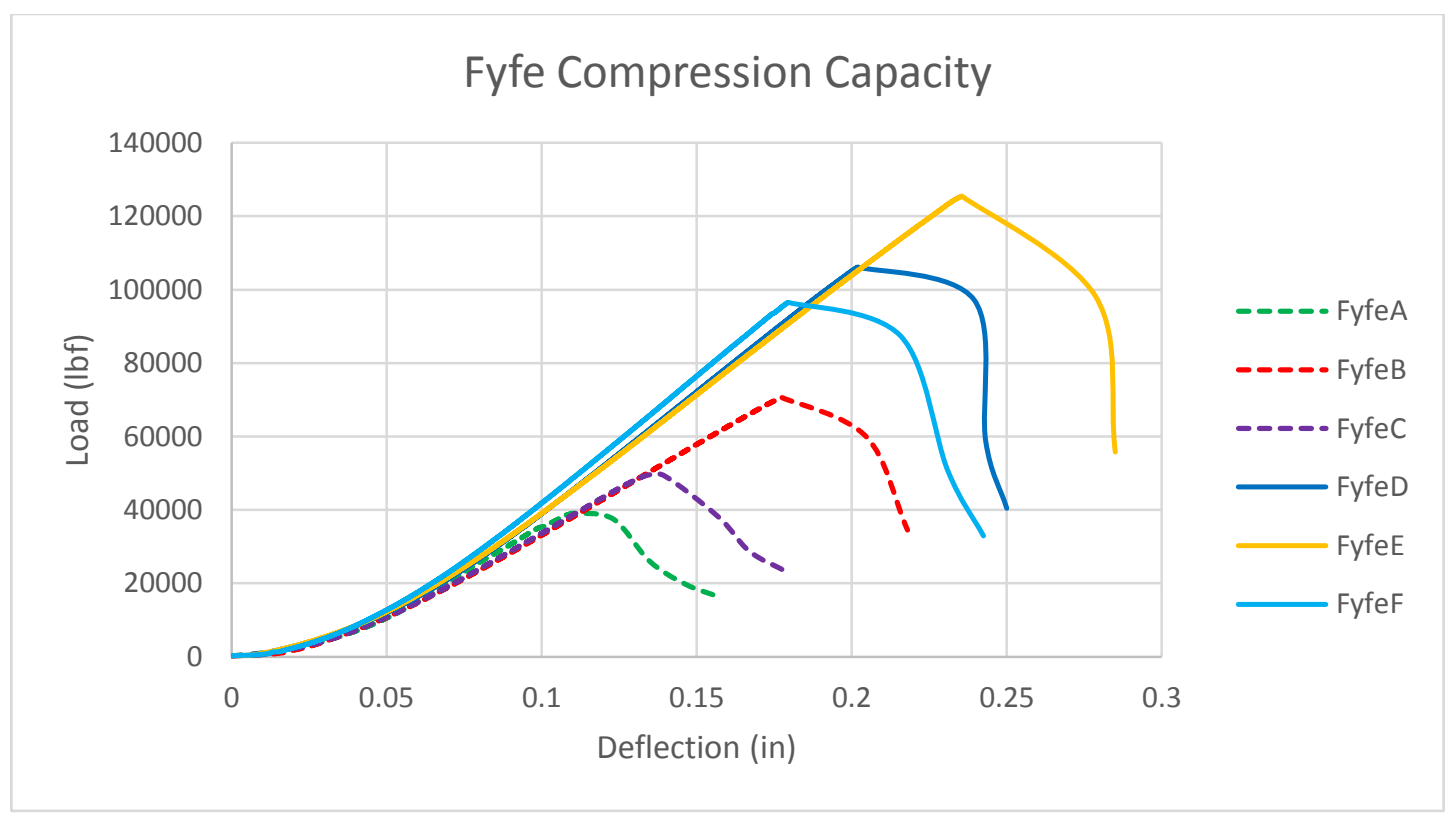

Figure 4-21: Plot of Fyfe compression load vs deflection 


\subsubsection{Sika Compression Results}

\subsubsection{Compressive capacity and strength of the Sika wraps}

The Sika wraps also displayed compressive failures in the fibers after layer separation, similar to the Fyfe system, as seen in Figure 4-22. The maximum compressive loads and stresses of the Sika shells are presented in Table 4-20 for three layers wrap and Table 4-21 for five layers of wrap. The maximum average stress for three layers was $13.8 \mathrm{ksi}$ and $14.1 \mathrm{ksi}$ for five layers. As noted, non-parallel bearing surfaces were present on half of the shells, which could have lowered capacity. More discussion of this influence will be provided in 4.4.6.3.

Table 4-20: Sika Three Layer Compression Results

\begin{tabular}{|c|c|c|c|c|}
\hline Specimen & $\begin{array}{c}\text { Area } \\
(\text { in^2) }\end{array}$ & Load (lbf) & Stress (psi) & FVF \\
\hline Sika A & 3.43 & 48,957 & 14,279 & $30 \%$ \\
\hline Sika B $^{1}$ & 3.43 & 26,688 & 7,784 & $30 \%$ \\
\hline Sika $C^{1}$ & 3.43 & 45,734 & 13,339 & $30 \%$ \\
\hline \multirow{2}{*}{\multicolumn{2}{|c|}{$\begin{array}{r}\text { Average: } \\
\text { COV: }\end{array}$}} & 47,346 & 13,809 & \\
\hline & & $3.4 \%$ & $3.4 \%$ & \\
\hline
\end{tabular}

${ }^{1}$ non-parellel testing surfaces (Average neglects Sika B)

Table 4-21: Sika Five Layer Compression Results

\begin{tabular}{|c|c|c|c|c|}
\hline Specimen & $\begin{array}{c}\text { Area } \\
(\text { in^^2) }\end{array}$ & Load (lbf) & $\begin{array}{c}\text { Stress } \\
\text { (psi) }\end{array}$ & FVF \\
\hline Sika $\mathrm{D}^{1}$ & 7.36 & 91,674 & 12,457 & $30 \%$ \\
\hline Sika E & 7.36 & 112,690 & 15,312 & $30 \%$ \\
\hline Sika F & 7.36 & 106,850 & 14,519 & $30 \%$ \\
\hline \multirow{2}{*}{\multicolumn{2}{|c|}{$\begin{array}{r}\text { Average: } \\
\text { COV: }\end{array}$}} & 109,770 & 14,096 & \\
\hline & & $2.7 \%$ & $2.7 \%$ & \\
\hline
\end{tabular}

${ }^{1}$ non-parellel testing surfaces (avg neglects Sika D) 


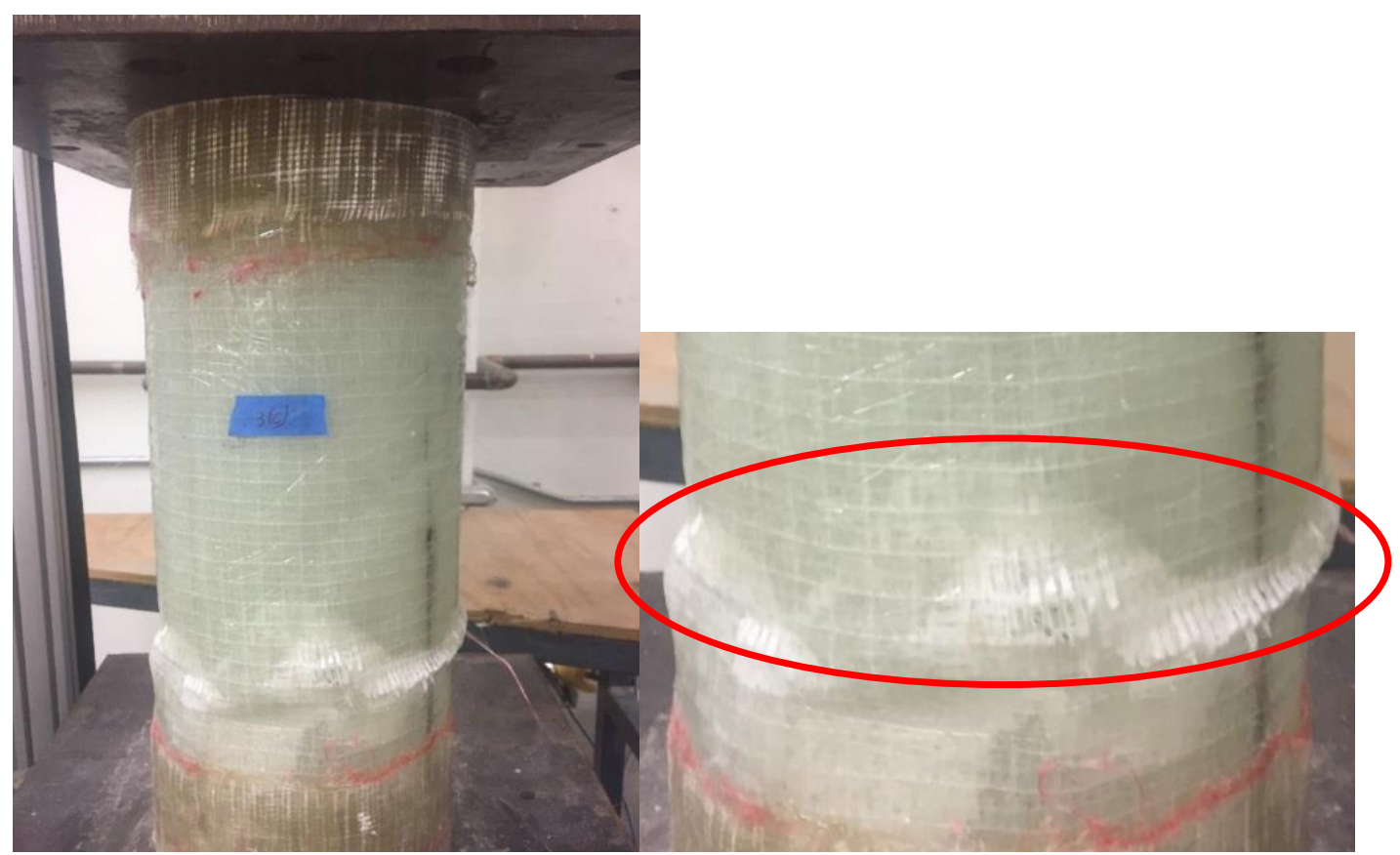

Figure 4-22: Typical compressive failure of sika system (Sika C) in the fibers

\subsubsection{Sika load versus deflection plots}

Plots of the load versus deflections of the Sika shells are shown in Figure 4-23. The Sika shells displayed very similar behaviors as to those of the Fyfe shells. Such a similarity is likely due to the similar nature of the Fyfe and Sika systems (same fabric type and epoxy resin).

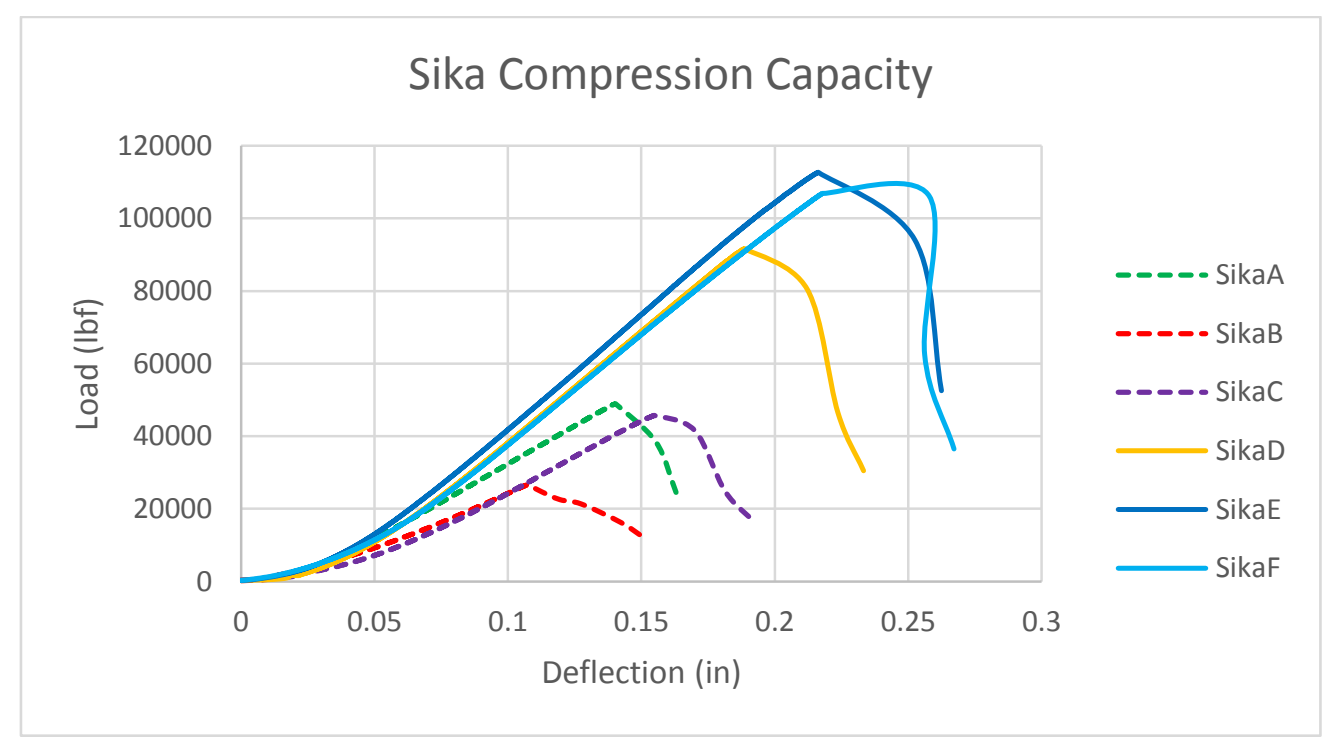

Figure 4-23: Sika Compression Capacities 


\subsubsection{Aquawrap® Compression Results}

Shells manufactured from the Aquawrap® system displayed classic elastic local buckling failure as shown in Figure 4-24. The layers appeared to debond from each other at failure. Once the load was removed, the shells returned to their original shapes. The maximum compressive loads and stresses of the Aquawrap® shells are presented in Table 4-22 for three layers wrap and Table 4-23 for five layers of wrap. The maximum average stress for three layers was $3.0 \mathrm{ksi}$ and $5.2 \mathrm{ksi}$ for five layers.

Table 4-22: Aquawrap ${ }^{\circledR}$ Three Layer Compression Results

\begin{tabular}{|c|c|c|c|c|}
\hline Specimen & $\begin{array}{c}\text { Area } \\
(\text { in^2) }\end{array}$ & $\begin{array}{c}\text { Load } \\
\text { (lbf) }\end{array}$ & $\begin{array}{c}\text { Stress } \\
\text { (psi) }\end{array}$ & FVF \\
\hline Aquawrap® A & 4.86 & 20,307 & 4,175 & $30 \%$ \\
\hline Aquawrap® B & 4.86 & 14,102 & 2,899 & $30 \%$ \\
\hline Aquawrap® $\mathrm{C}$ & 4.93 & 16,362 & 1,981 & $30 \%$ \\
\hline \multirow{2}{*}{\multicolumn{2}{|c|}{$\begin{array}{r}\text { Average: } \\
\text { COV: }\end{array}$}} & 16,924 & 3,018 & \\
\hline & & $15.2 \%$ & $29.8 \%$ & \\
\hline
\end{tabular}

Table 4-23: Aquawrap® Five Layer Compression Results

\begin{tabular}{|c|r|r|r|r|}
\hline Specimen & $\begin{array}{c}\text { Area } \\
\text { (in^2) }\end{array}$ & $\begin{array}{c}\text { Load } \\
\text { (lbf) }\end{array}$ & $\begin{array}{c}\text { Stress } \\
\text { (psi) }\end{array}$ & FVF \\
\hline Aquawrap ${ }^{\circledR}$ D & 8.26 & 27,882 & 5,655 & $30 \%$ \\
\hline Aquawrap ${ }^{\circledR}$ E & 7.92 & 35,399 & 4,468 & $30 \%$ \\
\hline Aquawrap® F & 8.04 & 45,049 & 5,606 & $30 \%$ \\
\hline & $\begin{array}{r}\text { Average: } \\
\text { COV: }\end{array}$ & 36,110 & $\mathbf{5 , 2 4 3}$ & \\
\cline { 3 - 5 } & & $19.5 \%$ & $10.5 \%$ & \\
\hline
\end{tabular}




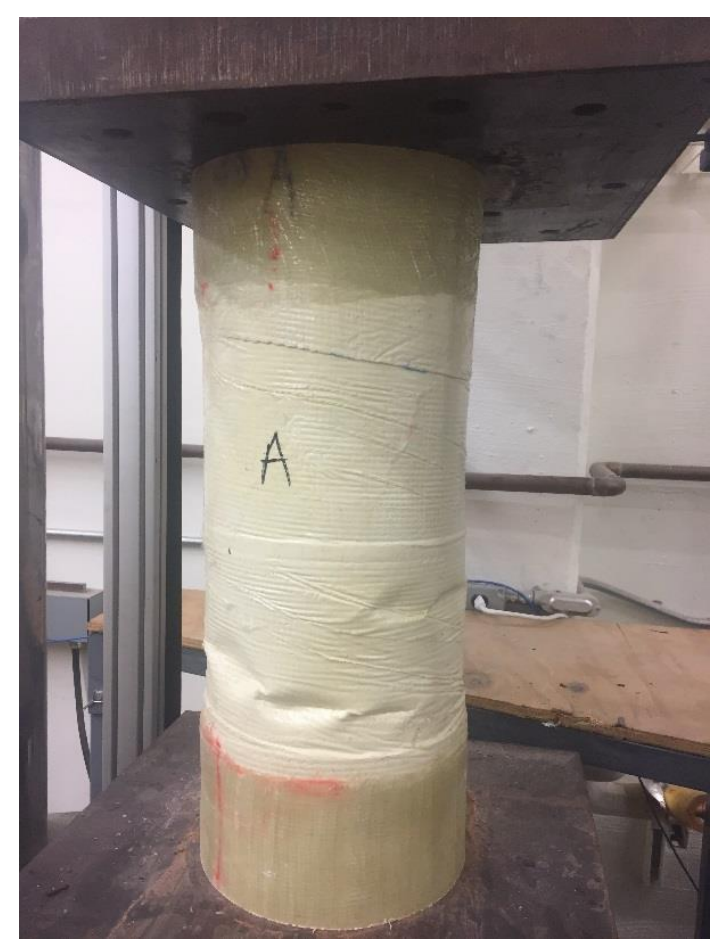

Figure 4-24: Elastic Local Buckling Failure of Aquawrap® (3 layers)

\subsubsection{Aquawrap ${ }^{\circledR}$ load versus deflection plots}

Plots of the load-deflection behaviors of the Aquawrap ${ }^{\circledR}$ shells are shown in Figure 4-25. Shells displayed a smoother peak versus the sharp drops in the Fyfe and Sika systems. The shape of the plots is likely due to the elastic local buckling failures between layers.

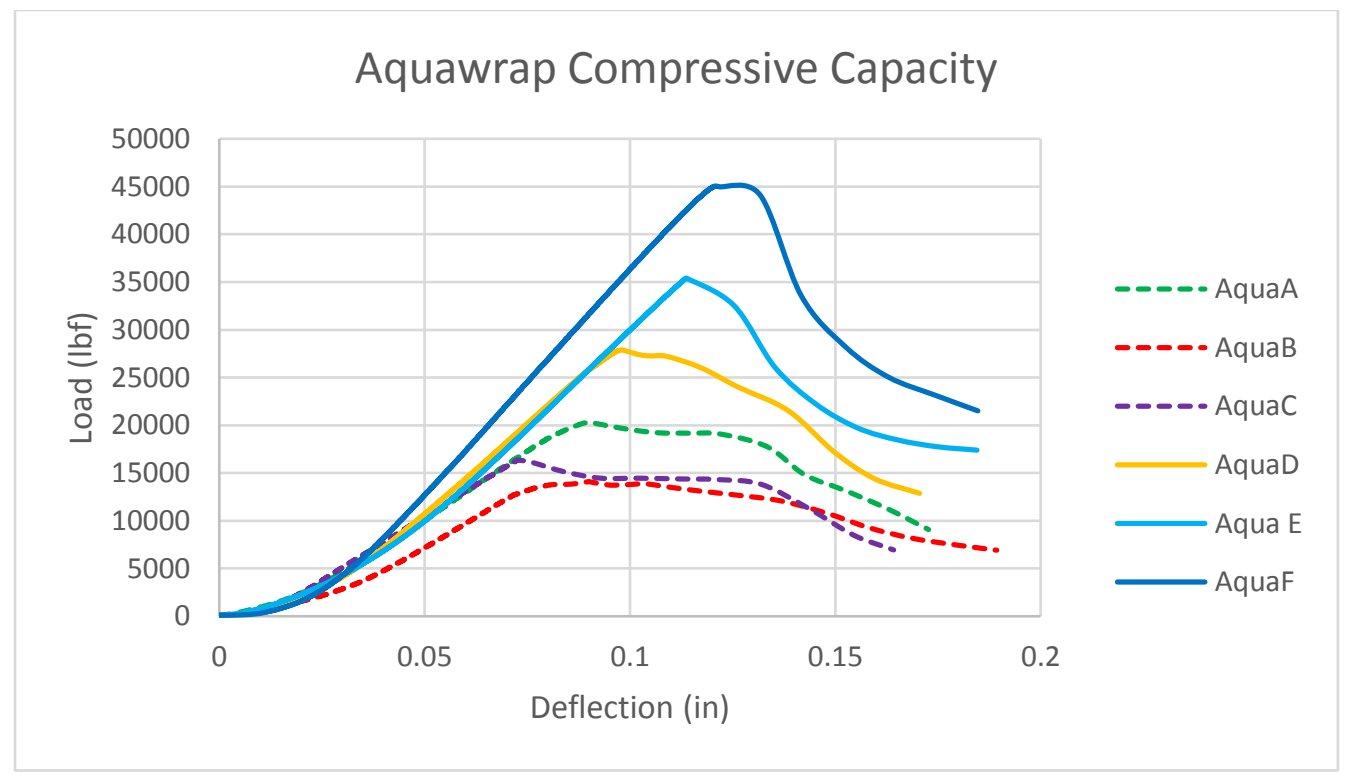


Figure 4-25: Aquawrap ${ }^{\circledR}$ Compression Capacities

\subsubsection{Phenolic Compression Results}

Phenolic shells all failed in compression of the fibers as shown in Figure 4-26. The maximum compressive loads and stresses of the phenolic shells are presented in Table 4-24 for three layers wrap and Table 4-25 for five layers of wrap. The maximum average stress for three layers was $5.2 \mathrm{ksi}$ and $7.7 \mathrm{ksi}$ for five layers. The failure mechanism was similar to the Fyfe and Sika systems, but as it was at a much lower load the visual damage is much less severe.

Table 4-24: Phenolic Three Layer Compression Results

\begin{tabular}{|c|c|c|c|c|}
\hline Specimen & $\begin{array}{c}\text { Area } \\
(\text { in^2) }\end{array}$ & $\begin{array}{c}\text { Load } \\
\text { (lbf) }\end{array}$ & $\begin{array}{c}\text { Stress } \\
(\mathrm{psi})\end{array}$ & FVF \\
\hline Phenolic A & 2.98 & 11,462 & 3,843 & $30 \%$ \\
\hline Phenolic B & 2.88 & 15,004 & 5,210 & $30 \%$ \\
\hline Phenolic C & 2.96 & 15,091 & 5,095 & $30 \%$ \\
\hline \multirow{2}{*}{\multicolumn{2}{|c|}{$\begin{array}{c}\text { Average: } \\
\text { COV: }\end{array}$}} & 15,048 & 5,152 & \\
\hline & & $0.3 \%$ & $1.1 \%$ & \\
\hline
\end{tabular}

Table 4-25: Phenolic Five Layer Compression Results

\begin{tabular}{|r|r|r|r|r|}
\hline Specimen & $\begin{array}{c}\text { Area } \\
\text { (in^2) }\end{array}$ & $\begin{array}{c}\text { Load } \\
\text { (lbf) }\end{array}$ & $\begin{array}{c}\text { Stress } \\
\text { (psi) }\end{array}$ & FVF \\
\hline Phenolic D & 4.98 & 37,390 & 7,515 & $30 \%$ \\
\hline Phenolic E & 4.98 & 36,380 & 7,312 & $30 \%$ \\
\hline Phenolic F & 4.98 & 40,563 & 8,153 & $30 \%$ \\
\hline \multicolumn{2}{|c|}{$\begin{array}{r}\text { Average: } \\
\text { COV: }\end{array}$} & 38,111 & $\mathbf{7 , 6 6 0}$ & \\
\cline { 2 - 5 } & & $4.7 \%$ & $4.7 \%$ & \\
\hline
\end{tabular}




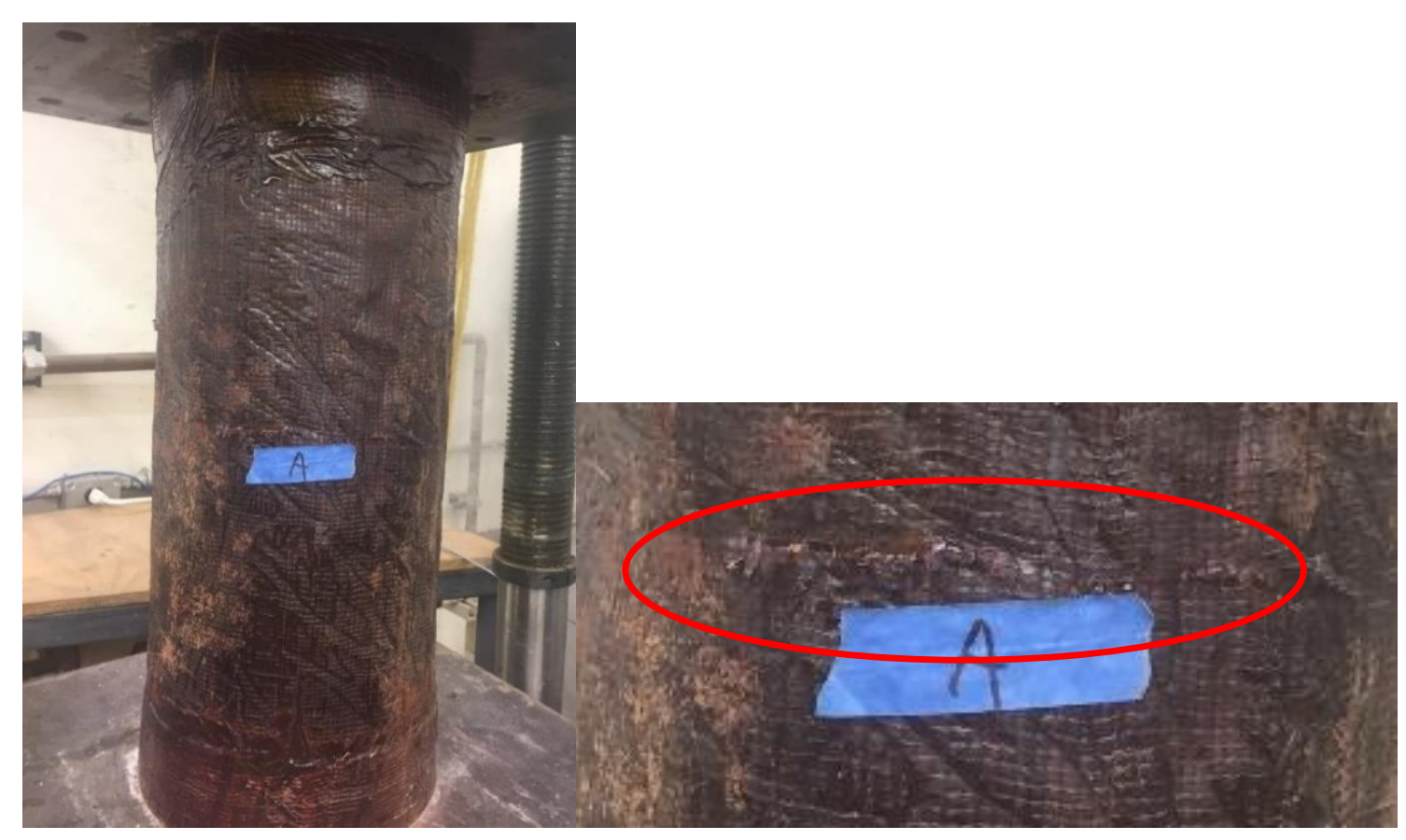

Figure 4-26: Typical compressive failure mode in the phenolic shell (Phenolic A)

\subsubsection{Phenolic load versus deflection plots}

Plots of the load versus deflection behaviors of the phenolic shells are shown in Figure 4-27. The plots display the same abrupt drops in load at failure present with the Fyfe and Sika which could correspond to the observed fiber failures. This shows that after the compression failure of the fibers the FRP shell has significantly reduced capacity. Coupled with the lack of visual indication of failure is concerning as it would be difficult to detect failure during a typical visual inspection even though a failed phenolic wrap might have significantly reduced capacity. 


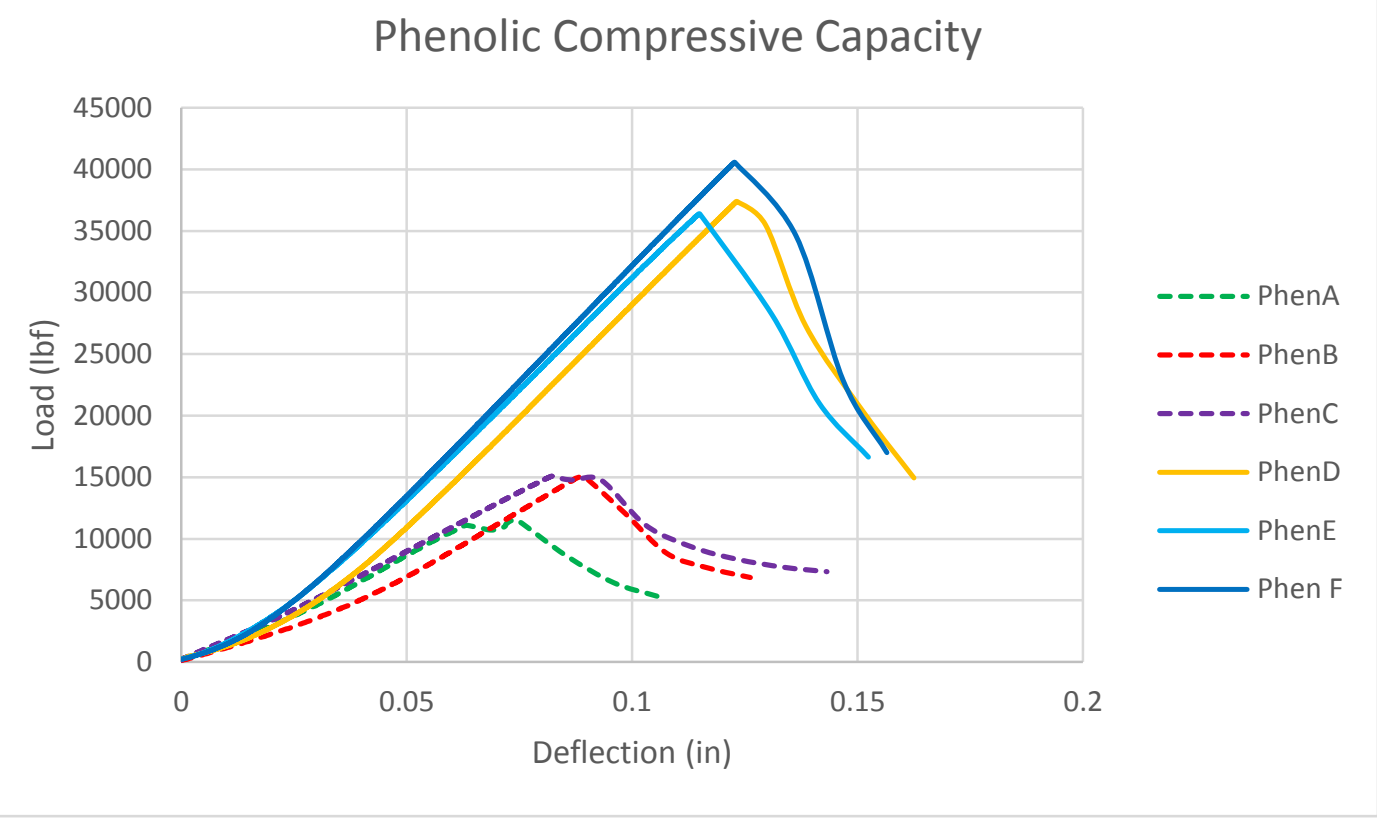

Figure 4-27: Phenolic Compression Capacities

\subsubsection{Coupon Testing Results}

Coupons constructed from three layers for each of the systems were tested. Results are included below. All coupons failed between the grips.

\subsubsection{Results and failure modes}

For the Fyfe systems, the average capacity was 59,719 psi as summarized in Table 4-26. The coupons displayed fiber failures as shown in Figure 4-28.

Table 4-26: Fyfe tensile strength

\begin{tabular}{|c|c|c|}
\hline Type & Load & Stress \\
\hline Fyfe 2 & 8529 & 64,918 \\
\hline Fyfe 3 & 8831 & 58,981 \\
\hline Fyfe 4 & 8638 & 57,565 \\
\hline Fyfe 5 & 8717 & 62,144 \\
\hline Fyfe 6 & 8392 & 62,199 \\
\hline Fyfe 8 & 8225 & 52,504 \\
\hline \multicolumn{2}{|c|}{ Average: } & $\mathbf{5 9 , 7 1 9}$ \\
\hline & COV: & $6.7 \%$ \\
\hline
\end{tabular}




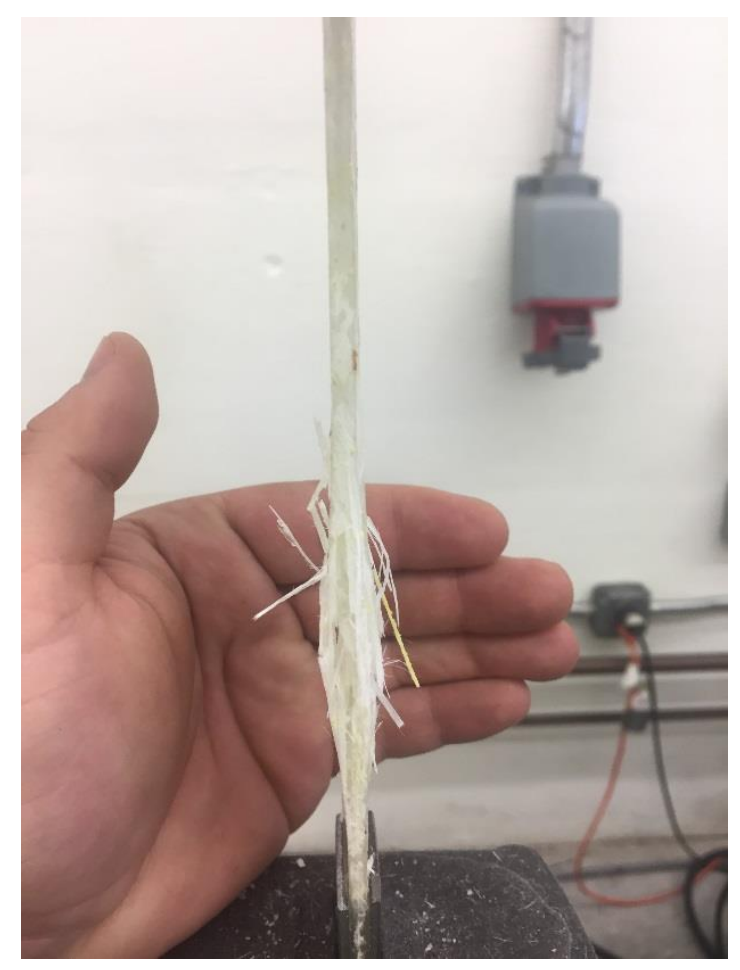

Figure 4-28: Fyfe coupon failure

For the Sika systems, the average capacity was 59,719 psi as summarized in Table 4-27. A small wrinkle (see Figure 4-29) was present. All failures occurred in the wrinkle (see Figure 4-30) and it is likely that such a wrinkle resulted in lower tensile strength values.

Table 4-27: Sika tensile strength

\begin{tabular}{|c|c|c|}
\hline Type & Load & Stress \\
\hline Sika 4 & 6841 & 44,234 \\
\hline Sika 5 & 7195 & 45,095 \\
\hline Sika 6 & 6908 & 41,740 \\
\hline Sika 7 & 7019 & 43,246 \\
\hline Sika 9 & 6531 & 37,740 \\
\hline \multicolumn{2}{|r|}{ Average: } & 43,579 \\
\hline & COV: & $5.9 \%$ \\
\hline
\end{tabular}




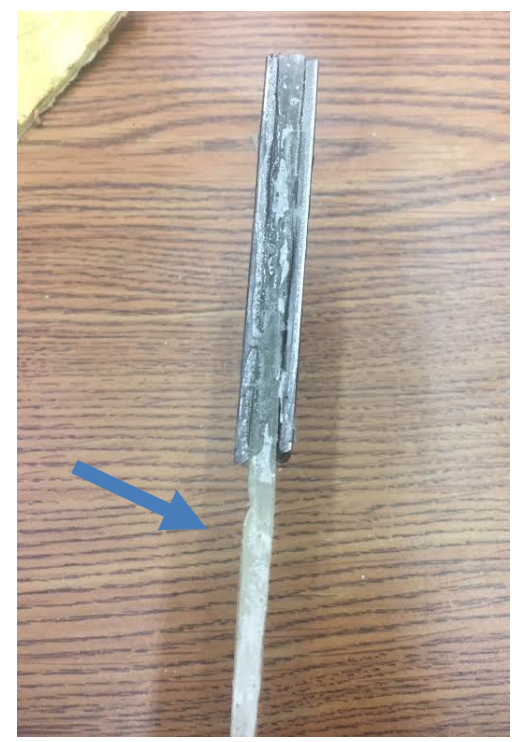

Figure 4-29: Sika coupon showing wrinkle

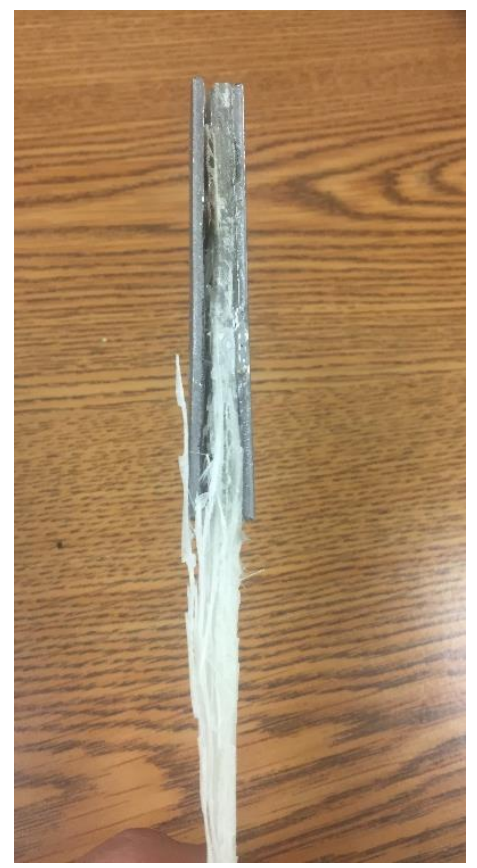

Figure 4-30: Sika Coupon Failure at wrinkle

For the Aquawrap® coupons, the average capacity was 35,436 psi as summarized in Table 4-28. The coupons displayed fiber failures, but the three layers de-bonded across nearly the entire length of the coupon after failure as shown in Figure 4-31. The de-bonding behavior suggests poor bonding between wrap layers. 
Table 4-28: Aquawrap ${ }^{\circledR}$ tensile strength

\begin{tabular}{|c|c|c|}
\hline Type & Load & Stress \\
\hline $\mathrm{Aq} 2$ & 3101 & 29,210 \\
\hline $\mathrm{Aq} 3$ & 3487 & 34,416 \\
\hline $\mathrm{Aq} 4$ & 3463 & 32,368 \\
\hline $\mathrm{Aq} 5$ & 3596 & 36,331 \\
\hline Aq 6 & 3868 & 39,006 \\
\hline $\mathrm{Aq} 7$ & 3871 & 41,285 \\
\hline \multicolumn{2}{|c|}{ Average Strength: } & 35,436 \\
\hline & COV: & $11.3 \%$ \\
\hline
\end{tabular}

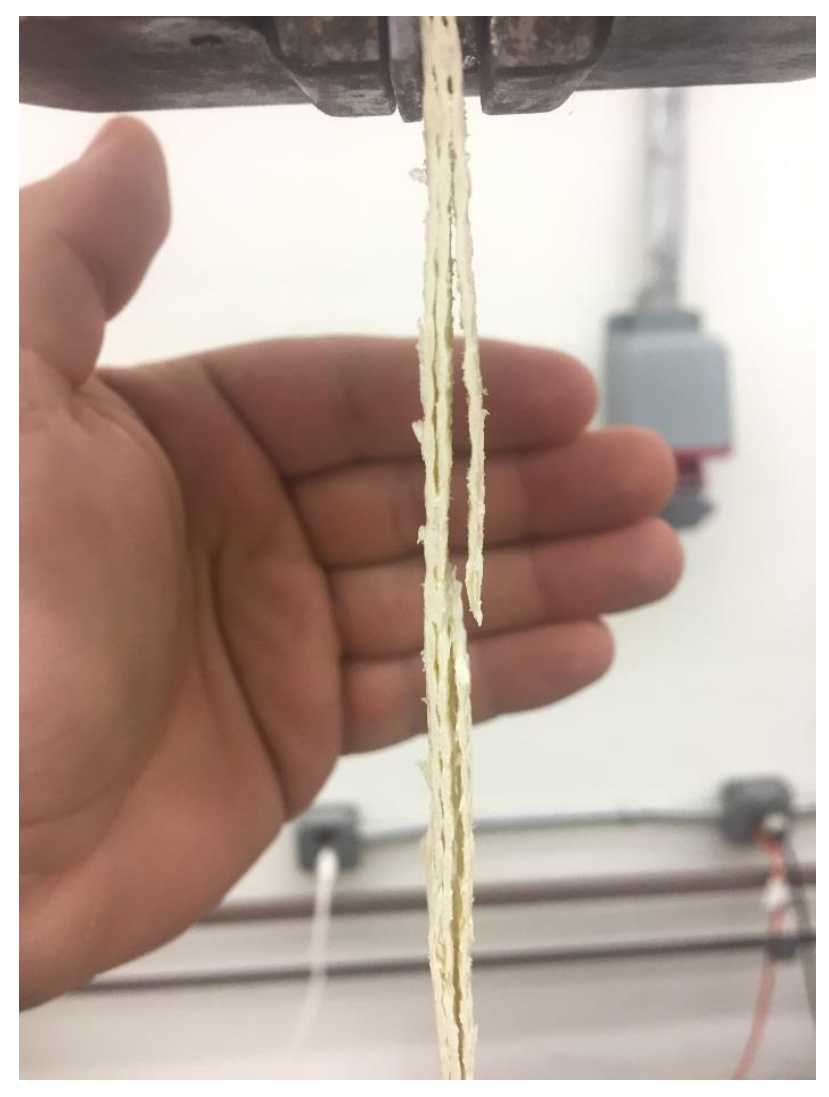

Figure 4-31: Aquawrap ${ }^{\circledR}$ coupon failure

For the phenolic coupons, the average tensile strength was 32,692 psi as summarized in Table 4-29. The coupons displayed fiber failure as shown in Figure 4-32. 
Table 4-29: Phenolic tensile strength

\begin{tabular}{|c|c|c|}
\hline Type & Load & Stress \\
\hline Phen 2 & 2840 & 32,977 \\
\hline Phen 3 & 2692 & 31,914 \\
\hline Phen 4 & 2325 & 27,463 \\
\hline Phen 5 & 3232 & 37,387 \\
\hline Phen 6 & 3036 & 33,589 \\
\hline Phen 7 & 2946 & 32,823 \\
\hline \multicolumn{2}{|r|}{ Average: } & 32,692 \\
\hline & COV: & $8.9 \%$ \\
\hline
\end{tabular}

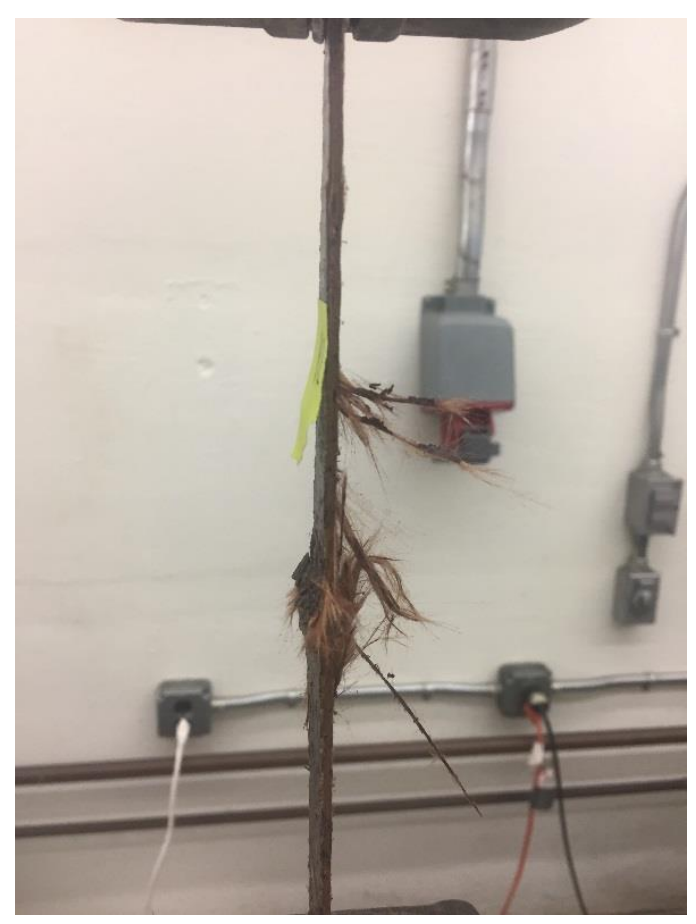

Figure 4-32: Phenolic Coupon Failure

Tensile coupon results are summarized in Table 4-30.

Table 4-30: Summarized Tensile Results

\begin{tabular}{|l|c|}
\hline \multicolumn{1}{|c|}{ System } & $\begin{array}{c}\text { Strength } \\
\text { (psi) }\end{array}$ \\
\hline Fyfe & 59,719 \\
\hline Sika & 43,579 \\
\hline Aquawrap $^{\circledR}$ & 35,436 \\
\hline Phenolic & 32,692 \\
\hline
\end{tabular}




\subsubsection{Discussion of Compression Results}

\subsubsection{Comparisons of compressive results}

Compression evaluations showed that Fyfe and Sika systems provided the highest capacities and strengths when compared to the other two systems. (Table 4-31 and Table 4-32). Overall increasing the number of wraps also increased the strength and capacity of the shells. (More layers $=$ higher strengths). Note that while the strength of the Fyfe and Sika shells increased slightly $(7.2 \%$ and $8 \%$ ) from three to five wraps, the Aqua and phenolic systems showed dramatic increases $(73.7 \%$ and $48.7 \%$ ) from three to five wraps. The sizable increase in strength corresponded fairly closely to the increase in the thickness of the shells (Aqua at $67 \%$ and Phenolic at $69 \%$ as shown in Table 4-33). However strength increases from additional wraps were between 2,000 to 3,000 psi for all systems. Therefore the relative percentage increases are more likely due to low compressive strength of the Aquawrap ${ }^{\circledR}$ and phenolic materials versus an actual improvement in the wrap capacity.

Table 4-31: Average compressive capacity by number of wraps (lbf)

\begin{tabular}{|l|c|r|r|}
\hline \multicolumn{1}{|c|}{ System } & $\begin{array}{c}\mathbf{3} \\
\text { Wraps }\end{array}$ & $\begin{array}{c}\text { 5 } \\
\text { Wraps }\end{array}$ & $\begin{array}{c}\text { Factor } \\
\text { Increased }\end{array}$ \\
\hline Fyfe & 59,962 & 115,810 & 1.93 \\
\hline Sika & 47,346 & 109,770 & 2.32 \\
\hline Aquawrap ${ }^{\circledR}$ & 16,924 & 36,110 & 2.13 \\
\hline Phenolic & 15,048 & 38,111 & 2.53 \\
\hline
\end{tabular}

Table 4-32: Average compressive strength by number of wraps (psi)

\begin{tabular}{|c|r|r|r|}
\hline System & $\begin{array}{c}\mathbf{3} \\
\text { Wraps }\end{array}$ & $\begin{array}{c}\text { 5 } \\
\text { Wraps }\end{array}$ & $\begin{array}{c}\text { Factor } \\
\text { Increased }\end{array}$ \\
\hline Fyfe & 14,511 & 15,551 & $7.2 \%$ \\
\hline Sika & 13,809 & 14,915 & $8.0 \%$ \\
\hline Aquawrap ${ }^{\circledR}$ & 3,018 & 5,243 & $73.7 \%$ \\
\hline Phenolic & 5,152 & 7,660 & $48.7 \%$ \\
\hline
\end{tabular}


Table 4-33: Average thicknesses of systems (inches)

\begin{tabular}{|l|c|c|r|}
\hline \multicolumn{1}{|c|}{ System } & $\begin{array}{c}\mathbf{3} \\
\text { layer }\end{array}$ & $\begin{array}{c}\mathbf{5} \\
\text { layer }\end{array}$ & $\begin{array}{c}\boldsymbol{\%} \\
\text { Increase }\end{array}$ \\
\hline Fyfe & 0.147 & 0.263 & $79 \%$ \\
\hline Sika & 0.123 & 0.260 & $112 \%$ \\
\hline Aquawrap ${ }^{\circledR}$ & 0.170 & 0.287 & $69 \%$ \\
\hline Phenolic & 0.105 & 0.175 & $67 \%$ \\
\hline
\end{tabular}

\subsubsection{Failure modes of compression shells}

For the Fyfe, Sika, and Phenolic systems, the shells failed universally in fiber crushing due to layer separation. The failure mode for the Aquawrap ${ }^{\circledR}$ shells was entirely in elastic local buckling and not in the fiber crushing. The elastic local buckling failure suggests poor adhesion between the layers in the system compared to the other systems.

When the load versus deflection behavior of the shells are plotted, the differences between failure modes are even more distinct (see Figure 4-33 and Figure 4-34). The plots for the Fyfe, Sika, and phenolic shells display consistent linear deformation until max capacities were reached. After the max capacities were reached, a sharp drop occurs in the plots which could correspond to the failure of the fibers. Plots of the Aquawrap® material displayed similar linear behavior as the load was applied until nearing the maximum load. After the maximum load, the plots show a slow decrease in load as the deformation continued to increase which would correspond with a more elastic local bucking failure mode versus sudden fiber fracture. 


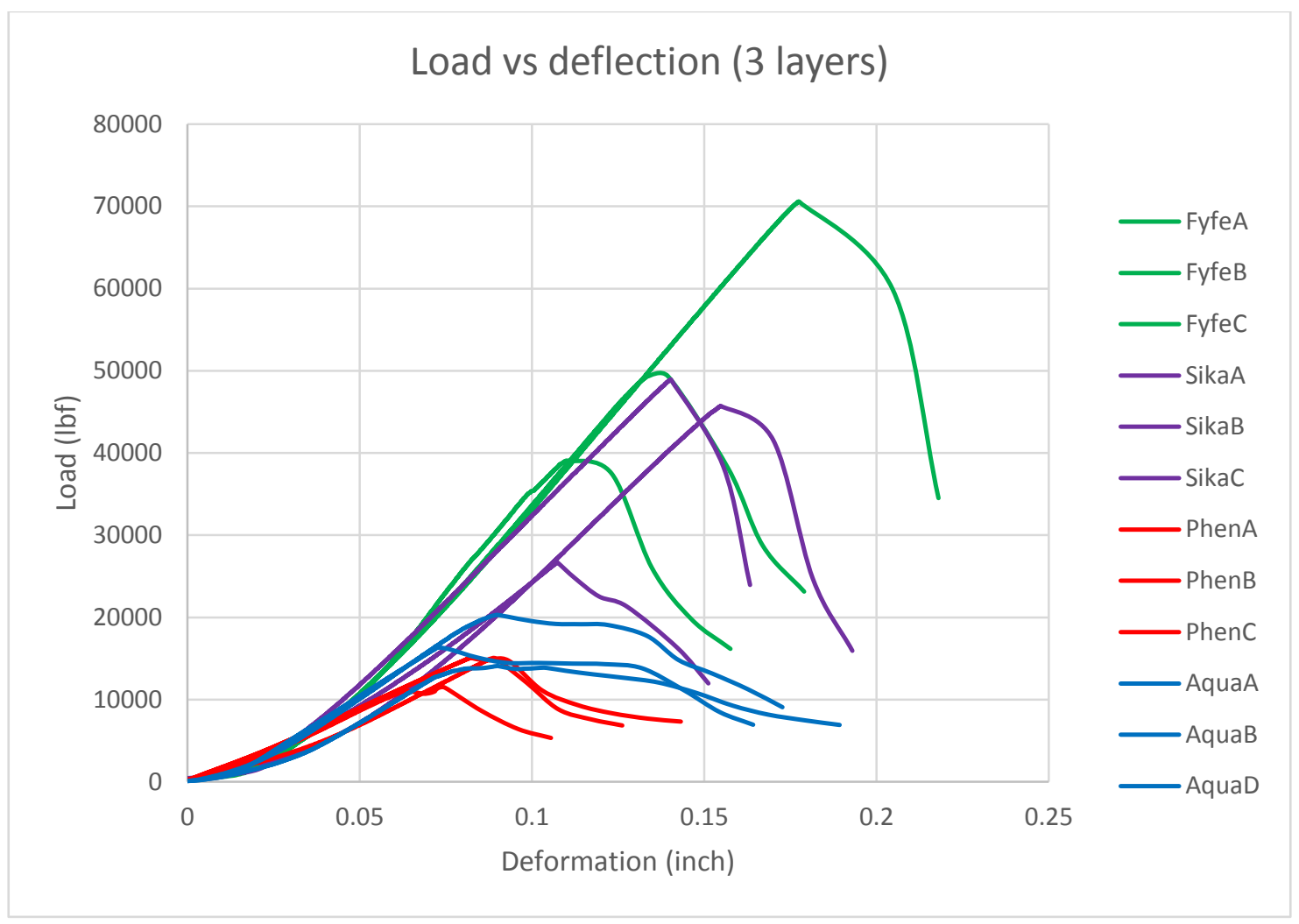

Figure 4-33: Load vs deflection for three layers of wrap

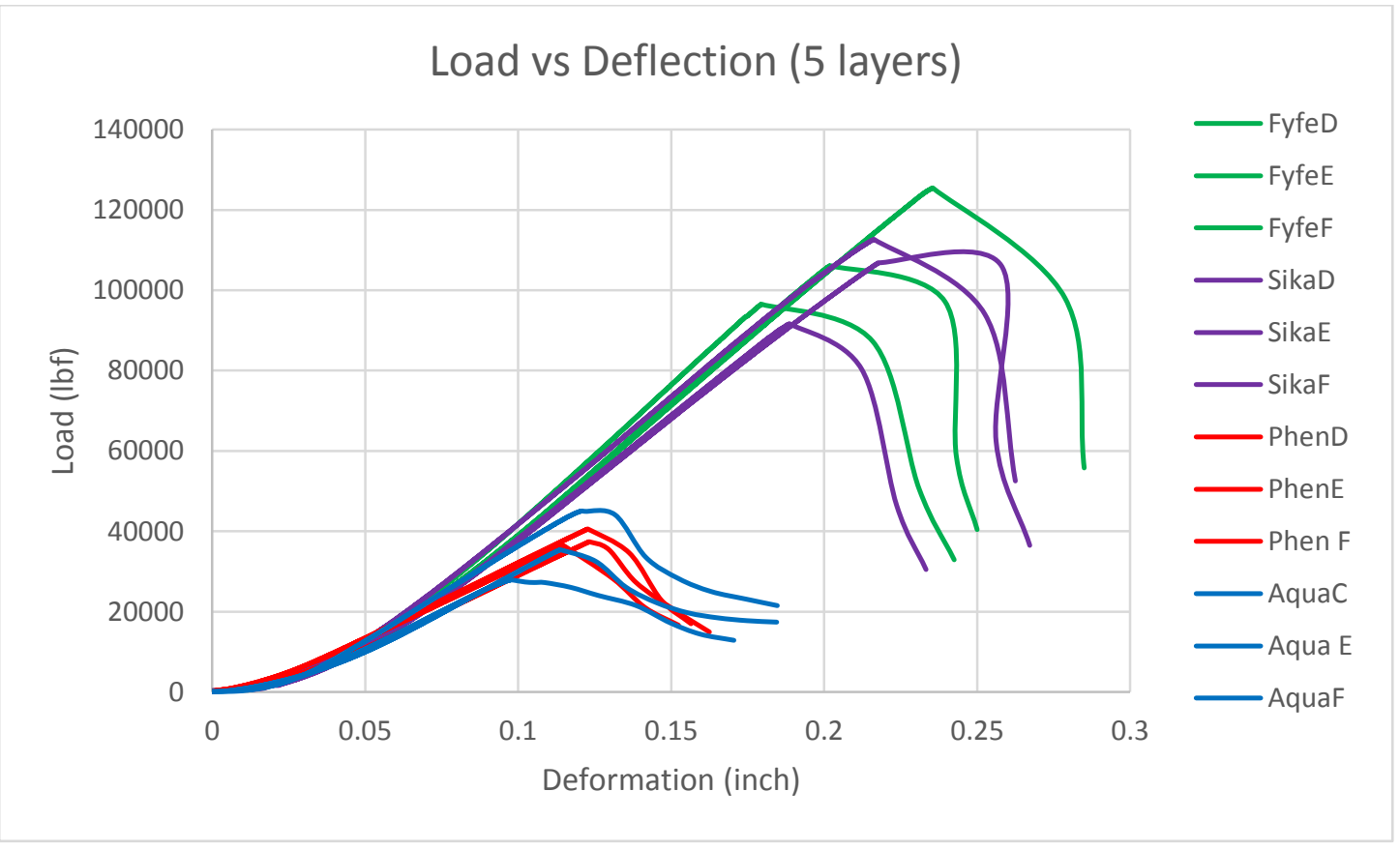

Figure 4-34: Load vs deflection for five layers of wrap 


\subsubsection{Influence from wrinkles caused by manufacturing}

As mentioned, the shells for the compression testing were manufactured through hand layup methods on PVC molds and secured for curing with stricture wrap. In general the manufacturing methods produced uniform shells with very few visible defections, but for the Fyfe system large wrinkles were present. The wrinkles were likely caused by two factors, oversaturation of a slick resin and stricture wrapping. The bond samples did not experiencing this wrinkling because the timber was less slippery then the smooth molds and less resin was used in the manufacturing of these samples.

The saturating epoxy used in the wrap had an extended work time, which allowed for deep fiber penetration, but it also resulted in resin with a slickness equivalent to petroleum jelly before curing. If too much resin was applied to saturate the fabric, the wrap became very difficult to secure to the mold or layers beneath. In some situations, this produced a loose wrap that could have potentially folded on itself creating the wrinkles. The issue could have been further exacerbated when the stricture wrap was applied. During stricture applications, the shells were wrapped tightly with uniform pressure until the entire shell was secured. Wrapping of the stricture could have easily pulled the oversaturated wrap onto itself forming the wrinkles. In either case, the wrinkles were present in Fyfe C, D, and F. In these samples, failure occurred in the wrinkles as seen in Figure 4-35 and Figure 4-36 and at lower strengths, though it is unknown if the failure initiated in the wrinkle or propagated to it. The wrinkles could have possibly created misalignment of the longitudinal fibers causing an off-axis load to be applied. The off-axis load likely resulted in a lowered strength values. 
From a manufacturing point of view, the other systems displayed fewer issues with wrinkling and other defects. Therefore more care should be taken with the Fyfe system to ensure proper resin saturation and stricture wrapping.

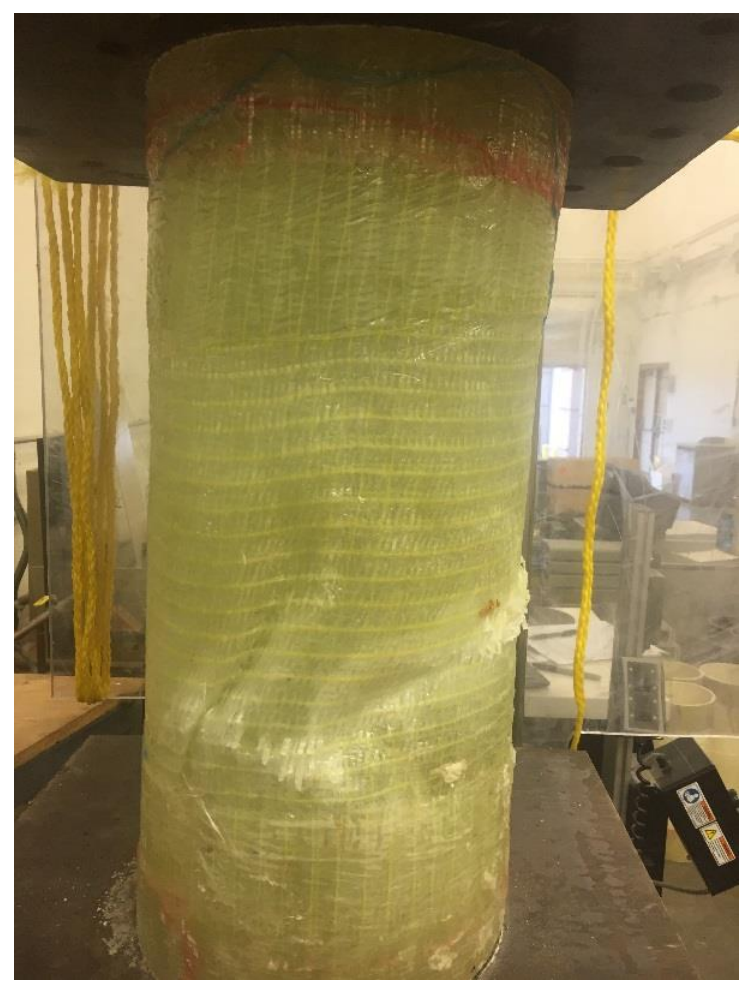

Figure 4-35: Fyfe $C$ with large wrinkle 


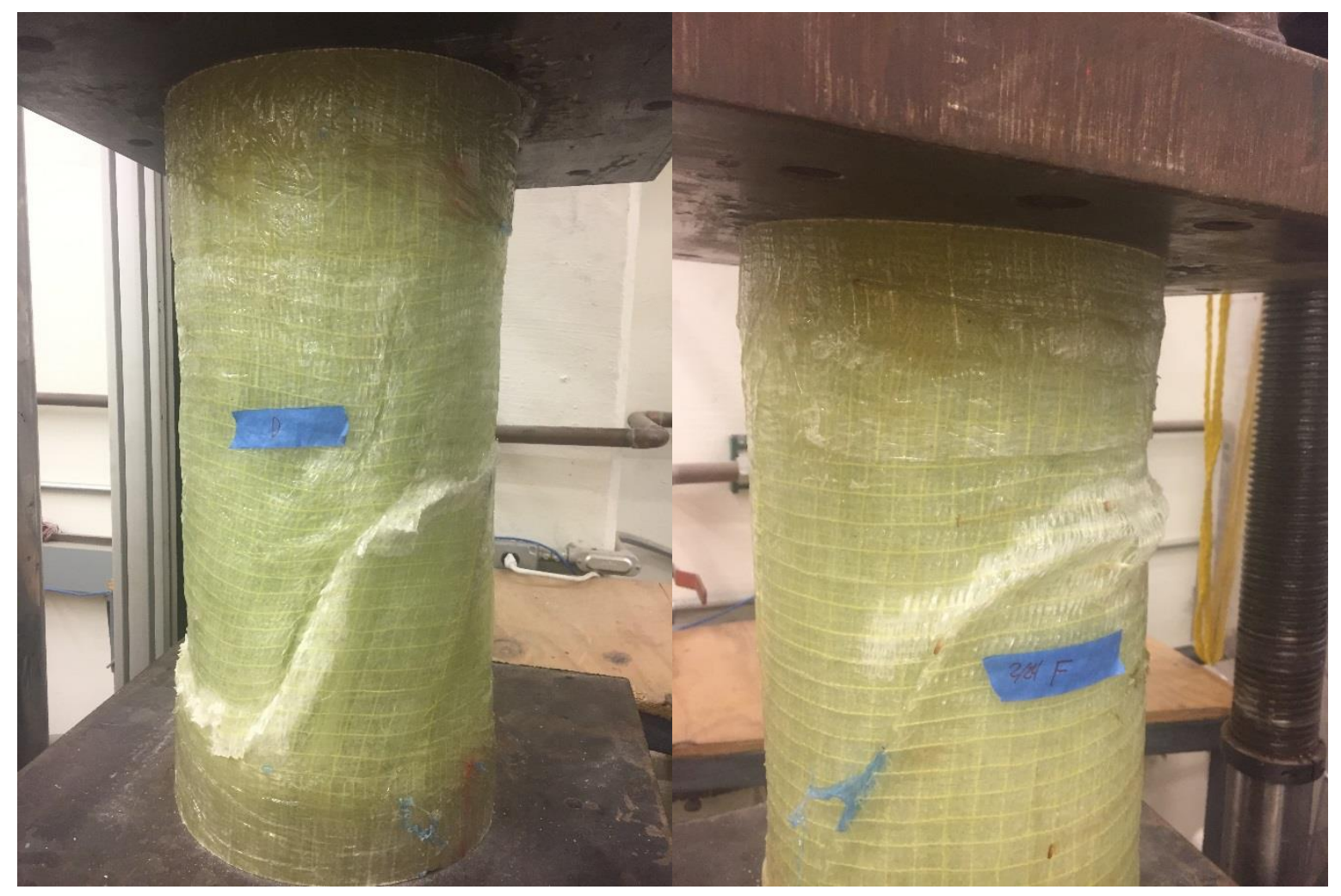

Figure 4-36: Fyfe D and Fyfe F wrinkles

\subsubsection{Influences of eccentricities}

All of the shells were trimmed before testing using a band saw to attempt to square off the ends parallel to one another. However, due to the end reinforcement changing the diameter, not all samples were trimmed with parallel surfaces which resulted in some unevenly distributed loading, mostly clearly illustrated in Figure 4-37 and Figure 4-38. However the testing head on machine was able to rotate (Figure 4-39) which helped to evenly distribute the load despite the non-parallel surfaces. 


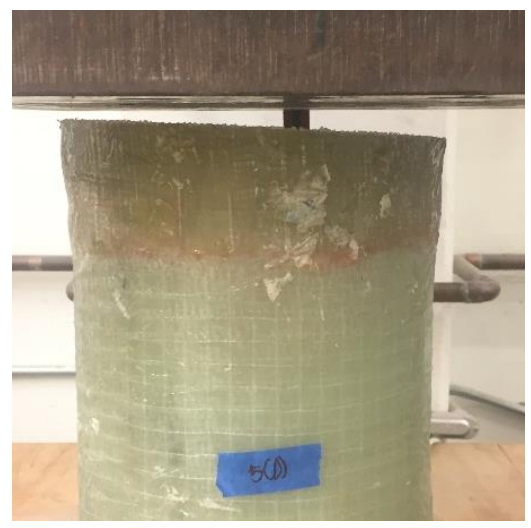

Figure 4-37: Lack of parallel bearing surface (Sika D)

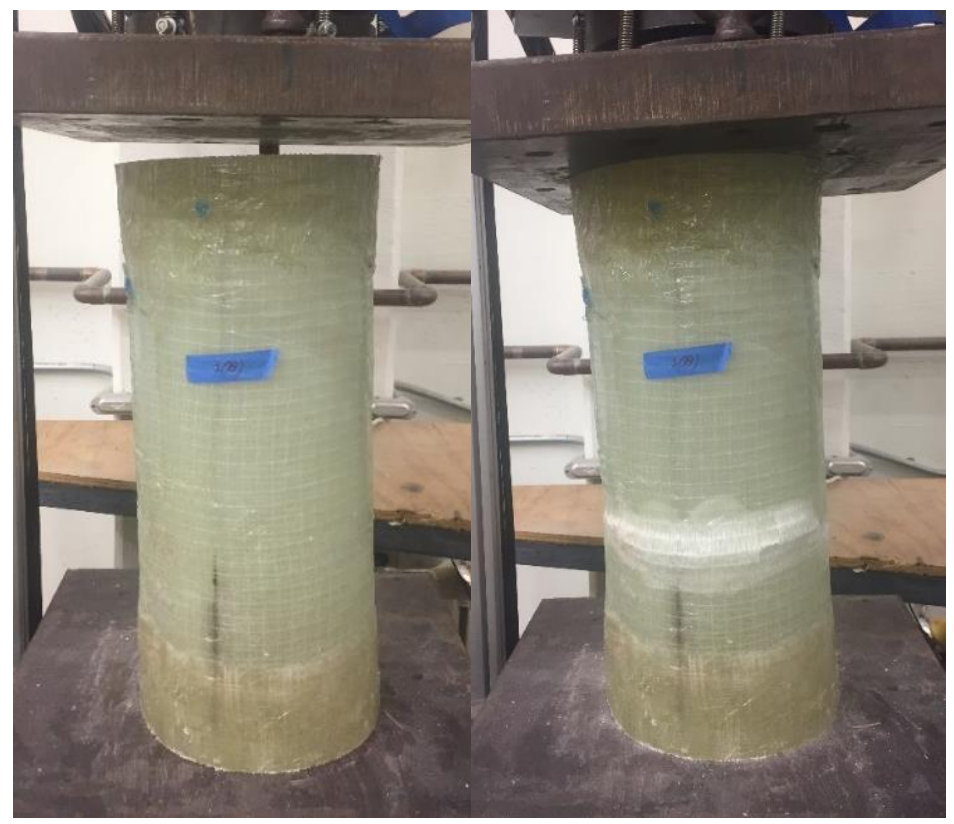

Figure 4-38: Failure from contributory eccentricity (Sika B)

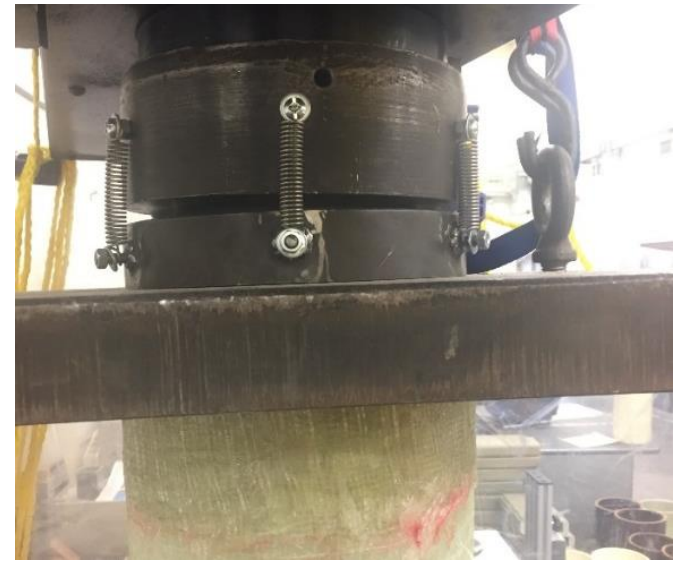

Figure 4-39: Testing head rotating to remove eccentricity 
On one occasion, a Fyfe sample was installed slightly off center in the machine which resulted in a relatively low compressive load. It was offset by roughly 2 to 3 inches as shown in Figure 4-40.

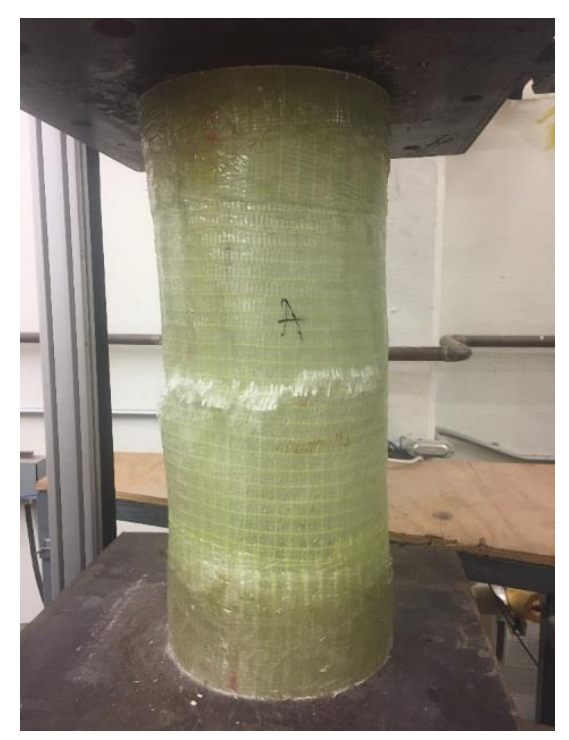

Figure 4-40: Fyfe A with incorrect placement in Instron (placed too far to the right)

The strength loss from the eccentricities is difficult to verify without additional testing and placing strain gages to collect strain data in the longitudinal and hoop directions. In the most extreme case, a reduction around $30 \%$ was found on Sika B compared to the other Sika samples. Sika B displayed an exceptionally non-parallel top surface that were beyond the adjustment limit of the machine. The remaining samples were within the adjustment range of the testing head and the eccentricities are not expected to have contributed significantly. However, more testing will be required with specific load off sets to properly calculate applied moments. The current results only supply very basic trends on the influences of eccentricities and the exact moments applied are not fully known. 


\subsubsection{Summary of influences}

The influences on the systems are summarized below in Table 4-34. These influences will be translated into knock down factors for future designs.

Table 4-34: Summary of influences on compressive capacity

\begin{tabular}{|l|l|l|}
\hline \multicolumn{1}{|c|}{ Factor } & \multicolumn{1}{c|}{ Samples } & \multicolumn{1}{c|}{ Influence } \\
\hline Wrinkles & Fyfe C, D, F & Reduced capacity by 20-30\% \\
\hline Off set Loading & Fyfe A & Reduced capacity by up to 45\% \\
\hline Uneven Surfaces & Sika B, D & Reduced capacity by 45, 20\%, respectively \\
\hline
\end{tabular}

\subsubsection{Comparing compression and tensile strengths}

The compression and tensile strengths for three layers of wrap for each systems are shown in Table 4-35. The Fyfe and Sika systems displayed the two highest strengths in both tension and compression while Aquawrap and phenolic systems displayed divergent behavior. The Aquawrap®, while higher than the phenolic in tension, displayed a comparably lower compressive capacity. To better understand this discrepancy, it is helpful to discuss the influence of fabric density (fiber volume fraction) on both compressive and tensile capacities for the systems.

Table 4-35: Compression vs tensile strengths compared (psi)

\begin{tabular}{|l|r|r|}
\hline System & Compression & Tensile \\
\hline Fyfe & 14,511 & 59,719 \\
\hline Sika & 13,809 & 43,579 \\
\hline Aqua & 3,018 & 35,436 \\
\hline Phenolic & 5,152 & 32,692 \\
\hline
\end{tabular}

\subsubsection{Fabric density related to compressive and tensile strength}

Both Fyfe and Sika fabrics used $27 \mathrm{oz} / \mathrm{yd}^{\wedge} 2$ uniaxial fabrics while Aquawrap ${ }^{\circledR}$ and phenolic used $22 \mathrm{oz} / \mathrm{yd}^{\wedge} 2$ and $18 \mathrm{oz} / \mathrm{yd}^{\wedge} 2$ bidirectional fabrics. For the wrap configurations 
selected, the fabric densities in the longitudinal direction were as shown in Table 4-36. The unidirectional fabrics were wrapped in a $0 / 90 / 0$ configuration, resulting in $2 / 3^{\text {rd }} \mathrm{s}$ of the fibers in the longitudinal direction while the bidirectional systems had equal fibers in each direction. If strength of the composite is assumed to be controlled by the fabric density, the Fyfe/Sika systems could be expected to have fairly high strengths followed in magnitude by Aquawrap ${ }^{\circledR}$ and then phenolic. However such is not the case.

Table 4-36: Fabric densities in longitudinal direction $\left(\mathrm{oz} / \mathrm{yd}^{\wedge}{ }^{\wedge}\right)$

\begin{tabular}{|l|r|r|}
\hline System & 3 layer & 5 layer \\
\hline Fyfe/Sika & 54 & 81 \\
\hline Aqua & 33 & 55 \\
\hline Phenol & 27 & 45 \\
\hline
\end{tabular}

The compressive strengths and fabric densities of phenolic and Aquawrap ${ }^{\circledR}$ specimens compared against the Fyfe material are given in Table 4-37. The longitudinal fabric density of the phenolic system is $50 \%$ to $55 \%$ of Fyfe/Sika systems for 3 and 5 layers, respectively. The compressive strengths tended to be in line with this, around 36\% for 3 layers and $50 \%$ for 5 layers. This suggests that 3 layers may be insufficient to develop the full compressive strength for the phenolic system. For Aquawrap ${ }^{\circledR}$, the longitudinal fabric density is $61 \%-67 \%$ of the Fyfe/Sika systems for three and five layers respectively. However, the Aquawrap ${ }^{\circledR}$ compressive strengths are $\sim 21 \%$ of the Fyfe/Sika systems for 3 layers and $\sim 34 \%$ for 5 layers.

Table 4-37: Comparing compressive strengths and fabric densities versus Fyfe

\begin{tabular}{|c|r|r|r|r|}
\hline \multirow{2}{*}{ System } & \multicolumn{2}{|c|}{ 3 Wraps } & \multicolumn{2}{c|}{ 5 Wraps } \\
\cline { 2 - 5 } & Strength & Density & Strength & Density \\
\hline Aquawrap® & $21 \%$ & $61 \%$ & $34 \%$ & $65 \%$ \\
\hline Phenolic & $36 \%$ & $50 \%$ & $49 \%$ & $56 \%$ \\
\hline
\end{tabular}


While the compressive data deviated from the relationship between fabric density and strength, the tensile strengths did not. The comparisons of fabric and tensile strengths for the phenolic and Aquawrap® tensile strengths with Fyfe fabric and tensile strengths are shown in Table 4-38. The relationship between the fabric density and tensile strength is more consistent for both systems versus compression behavior indicating that in tension, tensile strength is more controlled by the fabric density.

Table 4-38: Comparing tensile strengths and fabric densities versus Fyfe

\begin{tabular}{|c|r|r|}
\hline \multirow{2}{*}{ System } & \multicolumn{2}{|c|}{ 3 Wraps } \\
\cline { 2 - 3 } & Strength & Density \\
\hline Aquawrap ${ }^{\circledR}$ & $59 \%$ & $61 \%$ \\
\hline Phenolic & $55 \%$ & $50 \%$ \\
\hline
\end{tabular}

Therefore the relatively low strengths of Aquawrap ${ }^{\circledR}$ in compression compared to its fabric density and tensile behavior likely indicates a different compressive failure mode controlled by matrix debond and not fiber crushing. Such an observation corresponds well with the failure mode observed in 4.4.3 (Aquawrap ${ }^{\circledR}$ results). The Fyfe, Sika, and phenolic systems displayed failure modes that were directly proportional on their respective fabric densities.

\subsubsection{Stiffness behavior}

Up to a certain point, the tensile and compressive strengths should have similar stress-strain behavior as the modulus is a material property. While no strain gages were used in this study, the global strain (change in length over original length) was calculated based off of the position data obtained from the Instron machines. The "E" value was determined based off of $20 \%$ of ultimate failure and the ultimate for each as summarized below in Table 4-39. Due to the settling of the machines, the strains calculated in the manner are not considered accurate but are given for a rough estimation of the modulus. Fyfe modulus are relatively close for both compression and tension 
(13\% differenc). The Sika coupon moduli are much lower than expected by nearly $1 / 2$. The low tensile modulus is likely due to wrinkle mentioned previously which reduced the strength of the material. The Aquawrap tensile modulus was slightly higher than its compressive modulus which correlates well with revealed data (ei relatively low compressive strengths compared to other systems). The phenolic system had a very consistent modulus for both materials.

Table 4-39: Average moduli based on compression and tension

\begin{tabular}{|l|c|r|r|r|}
\hline \multirow{2}{*}{ System } & \multicolumn{2}{|c|}{ Compression } & \multicolumn{2}{c|}{ Tension } \\
\cline { 2 - 5 } & $\begin{array}{c}\text { Modulus } \\
\text { (ksi) }\end{array}$ & \multicolumn{1}{c|}{ COV } & $\begin{array}{c}\text { Modulus } \\
\text { (ksi) }\end{array}$ & COV \\
\hline Fyfe & 2296.5 & $6.8 \%$ & 2009.4 & $6.5 \%$ \\
\hline Sika & 2212.2 & $15.8 \%$ & 1211 & $11.7 \%$ \\
\hline Aquawrap $^{\circledR}$ & 1136.4 & $11.2 \%$ & 1263.7 & $8.7 \%$ \\
\hline Phenolic $^{2}$ & 1412.8 & $3.9 \%$ & 1439.1 & $6.5 \%$ \\
\hline
\end{tabular}

By comparing the relative stiffness, it can be shown that theoretically, the compression samples could develop strengths equivalent to the tension results. It is likely that voids created during the hand lay-up process and eccentric loads applied served to fail the material prematurely. If small scale compressive coupon specimens were created for ASTM compression testing, it is very likely that higher compressive strengths could be reached for these specimens as this would eliminate many of the strength reducing factors that come into play during scale up.

\subsubsection{Differences between published values and tested values.}

The tensile coupon testing conducted herein had consistently lower values than the published tensile properties of the manufacturers as shown in Table 4-40Error! Reference source not found.. Both Fyfe and Sika publish design values that exceed the tested values obtained herein (66,720 psi and 77,100 respectively). The compressive strengths from the full size samples are 
extremely divergent from published values (Fyfe and Aquawrap ${ }^{\circledR}$ do not publish compressive strengths, but they are expected to be similar to tensile strengths).

Table 4-40: Variations in Tensile Coupon Testing Results

\begin{tabular}{|l|r|r|r|}
\hline System & \multicolumn{1}{|c|}{ Tested } & Published & Difference \\
\hline Fyfe & 59,719 & 83,400 & $-28 \%$ \\
\hline Sika & 43,579 & 88,800 & $-51 \%$ \\
\hline Aqua & 35,436 & 45,400 & $-21 \%$ \\
\hline Phenolic & 32,692 & N/A & \\
\hline
\end{tabular}

The lower tensile strengths $(30 \%<)$ are expected for the Fyfe and Sika samples as only 2/3 of the fibers are oriented in the longitudinal direction. The additional low value in the Sika samples is likely due to the wrinkle present on the coupons. Further differences between the results in this study and the published values are likely due to the variations inherent in the hand layup process (which could have produced a lower fiber volume fraction in the composite). There were also likely differences in the curing environments as manufacturers often utilize a higher temperature post cure. However, these differences are likely to be replicated in the field given the complexity of field installation. Thus, appropriate knock-down factors should be developed for design to use any manufacturer supplied data.

The compressive strengths are much lower than design values again due in part to $2 / 3$ of the fibers running in the longitudinal directions. While the results from this study are based on large scale hand layup specimens, the design values were conducted according to ASTM standards on very small coupons. The coupon level would have far fewer deformities and voids present in the composite and no eccentric loadings present. As demonstrated previously by examining the modulus of the systems, the full scale compressive strengths could theoretically reach this value if no layer separation occurred. However full scale specimens will not be able to reach these design values. Therefore it would be more conservative to use the values determined from this study for 
designing full scale repairs in the field as the same limitations from the layup process will be present.

Table 4-41: Compressive Strengths

\begin{tabular}{|l|r|r|r|}
\hline System & \multicolumn{1}{|c|}{ Tested } & Published & Difference \\
\hline Fyfe & 14,511 & $83,400 *$ & $-83 \%$ \\
\hline Sika & 13,809 & 86,800 & $-85 \%$ \\
\hline Aqua & 3,018 & $45,400 *$ & $-93 \%$ \\
\hline Phenolic & 5,152 & N/A & \\
\hline
\end{tabular}

* Values from tensile testing and assumed to be similar

\subsubsection{Comparison with required axial capacity of the piles}

As with the bonding tests, the compressive evaluations were conducted to confirm if the compressive capacities of the systems could match the capacity for an equivalent new timber pile (for 10" diameter, 94-98 kips, [29] ). From the tests, it would seem that five wraps of the Fyfe and Sika systems (115.8 kips and 109.8 kips) could provide this capacity, but the other systems would not likely provided the needed capacity with the number of wraps used. If the trends presented herein continue, both the Aquawrap ${ }^{\circledR}$ and Phenolic systems would need in excess of 10 layers of FRP to fully carry the load originally carried by the pile. However, further testing would be required to determine the exact number of wraps as it is not clear if the relationship to number of wraps and overall capacity would continue to increase linearly.

\subsection{Full Scale Rehabilitation Simulation}

Rehabilitation simulation samples using three wraps with 12 " bond lengths on timber piles with an 18" gap were tested for Fyfe and phenolic systems. Results are summarized in 4.5.1 and discussed in 4.5.2. 


\subsubsection{Summary of rehabilitation results}

During wrapping of these specimens, long widths of fabric were used which resulted in at minimum of 12 " of overlap. After three layers of fabric, such overlaps were equivalent to at-least one additional layer of fabric bringing the actual number of layers to closer to four layers. After testing, the samples were cut and the actual thicknesses of the wraps were directly measured for accurate stress calculations. The failure mode for both systems was consistently in compression of the shell and not bond slippage. For the Fyfe specimen, failure occurred at the transition between the wrap and the timbers. Causes for this type of failure will be discussed below in 4.5.2.1. The phenolic specimen failed in the center of the specimen, a similar failure mode experienced by the phenolic compression samples. Shown in Table 4-42 are the summarized results for the piles evaluated. Eccentricities from the manufacturing methods likely have caused a reduced capacity in the samples, but were within the adjustment of the testing head and are within the normal out of plumb levels seen in field installations. However, the overall length of the samples (60 inches long) would result in even small eccentricities creating significant bending. Plots of the load versus deflection behavior of the load were given in Figure 4-41. Photos of the failure modes are provided in Figure 4-42 and Figure 4-43.

Table 4-42: Summary of Rehab Simulations

\begin{tabular}{|l|c|c|c|c|c|c|}
\hline System & $\begin{array}{c}\text { Ultimate } \\
\text { Load } \\
\text { (lbf) }\end{array}$ & $\begin{array}{c}\text { Compress } \\
\text { Area } \\
\text { (in^2) }\end{array}$ & $\begin{array}{c}\text { Compress } \\
\text { Stress } \\
\text { (psi) }\end{array}$ & $\begin{array}{c}\text { Bond } \\
\text { Area } \\
\text { (in^2) }\end{array}$ & $\begin{array}{c}\text { Bond } \\
\text { Stress } \\
\text { (psi) }\end{array}$ & Failure Mode \\
\hline Fyfe & 49,733 & 6.46 & 7,698 & 379 & 131 & Compression \\
\hline Phenolic & 20,953 & 4.33 & 4,843 & 361 & 58 & Compression \\
\hline
\end{tabular}




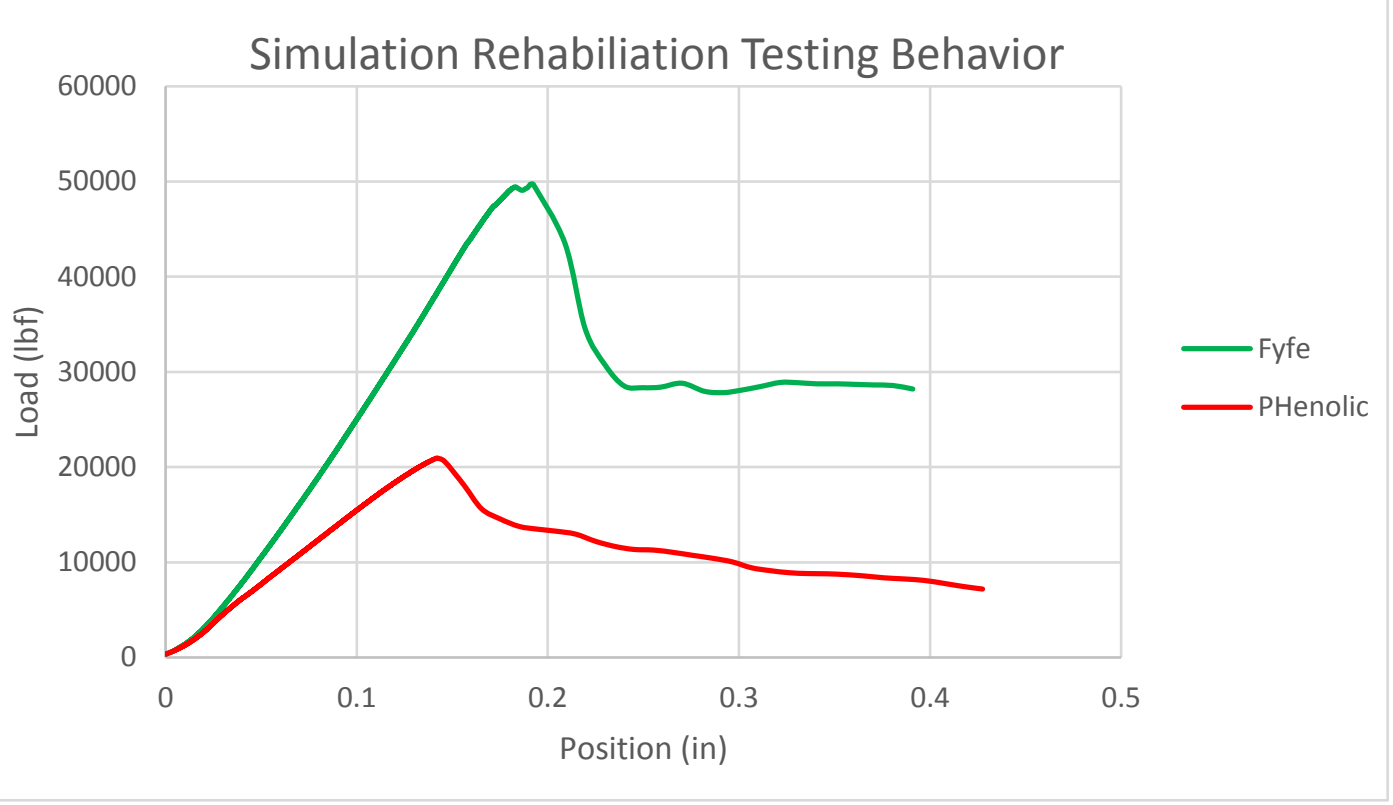

Figure 4-41: Load versus position behavior of wraps on the piles

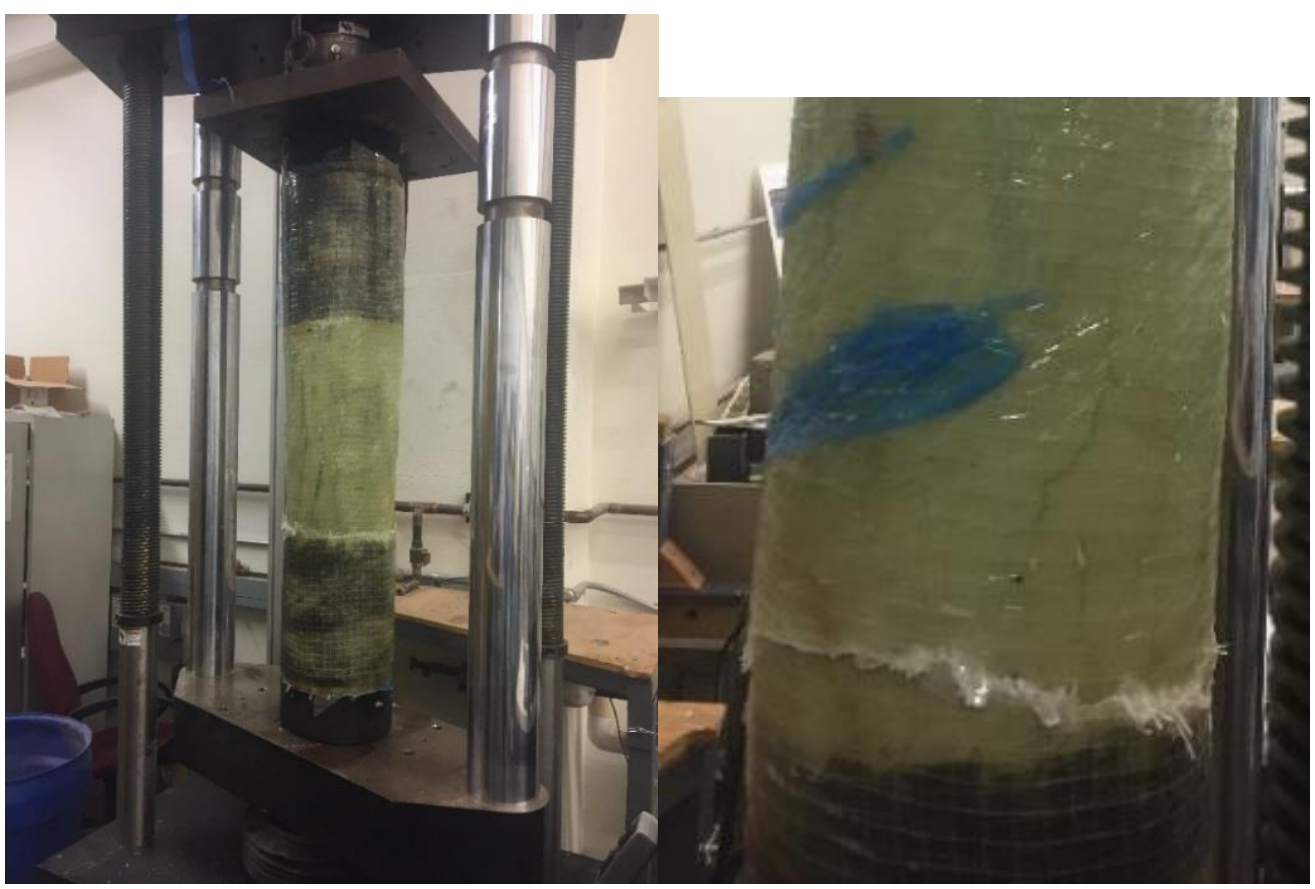

Figure 4-42: Fyfe simulated rehab failure 


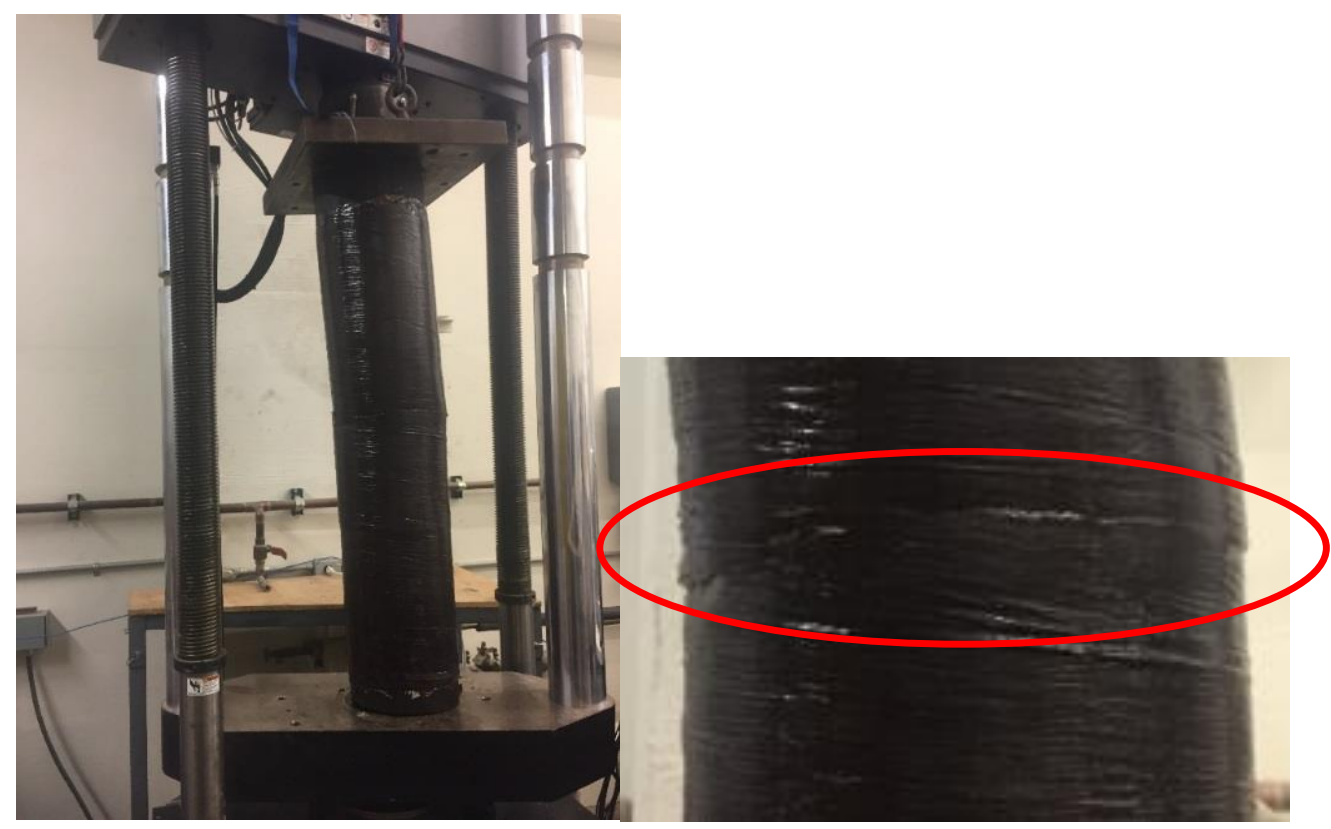

Figure 4-43: Phenolic simulated rehab failure

\subsubsection{Discussion of full scale rehabilitation simulations results}

\subsubsection{Comparison with compressive and bond strengths}

The compressive strengths obtained from the rehabilitation simulation testing were compared with the compression testing results as shown in Table 4-43.

Table 4-43: Comparison with compression strength (psi)

\begin{tabular}{|l|c|c|c|c|}
\hline \multirow{2}{*}{ System } & \multicolumn{2}{|c|}{ Compression } & \multicolumn{2}{c|}{ Rehab } \\
\cline { 2 - 5 } & $\begin{array}{c}\text { Thickness } \\
\text { (in) }\end{array}$ & $\begin{array}{c}\text { Strength } \\
\text { (psi) }\end{array}$ & $\begin{array}{c}\text { Thickness } \\
\text { (in) }\end{array}$ & $\begin{array}{c}\text { Strength } \\
\text { (psi) }\end{array}$ \\
\hline Fyfe & 0.147 & 14,511 & 0.200 & 7,698 \\
\hline Phenolic & 0.105 & 5,152 & 0.142 & 4,843 \\
\hline
\end{tabular}

Compared to compression testing results, the Fyfe specimen showed a $47 \%$ reduction in compressive strength even with the additional thickness. As previously stated, the Fyfe specimen failed in the transition zone between the timber and the hollowed portion of the wrap. The transition zone likely caused large stress concentrations to develop which would account for this 
lowered strength. To counter this, it would be reasonable to apply additional reinforcing wraps in the transition zone in the failure. Furthermore a visual inspection of the wrap showed that the acetone used to melt out the foam degraded the resin matrix in this zone further contributing to strength reduction. (Note: degradation from acetone will not be an issue encountered in the field). As mentioned, the eccentricities present also contributed to the reduced strengths.

Phenolic compressive strengths were reduced by $6 \%$. As noted in the compression testing, the phenolic compressive strengths increased with the wrap thickness which would account for less reduction in strength compared to the Fyfe system. The eccentricity present likely accounted for the majority of the loss in strength. The exact amount of compression strength reduction from eccentricity will be estimated during future evaluations of the influence of eccentricity on compressive strengths.

The bond stresses developed did not exceed the bond strength of the specimens as shown in Table 4-44. The stress developed on the phenolic rehab, however, was fairly close to the average phenolic bond strength.

Table 4-44: Comparison with bond strength (psi)

\begin{tabular}{|c|c|c|}
\hline System & Bond & $\begin{array}{c}\text { Rehab } \\
\text { (did not fail in bond) }\end{array}$ \\
\hline Fyfe & 240 & 131 \\
\hline Phenolic & 64 & 58 \\
\hline
\end{tabular}

\subsubsection{Conclusions}

The simulation rehabilitations suggest that the individual compression and bond tests are likely suitable test methods to replicate the field conditions for the Fyfe system, but are not as conclusive regarding the phenolic. The differences in the phenolic could be due the hand lay-up manufacturing method producing a variable product. Additional samples are prepped for wrapping 
with Aquawrap ${ }^{\circledR}$ and the Sika systems, and test of these samples will shed more light on the validity of the test methods. 


\section{CHAPTER 5 CONCLUSIONS AND RECOMMENDATIONS}

\subsection{Bond Strength Evaluations}

Greater bonding area resulted in higher bond capacity, but lowered bond strength in terms of pounds per square inch of bond. The Fyfe system displayed the best bond strengths for both push out and pull off tests indicating a superior resin system. A possible explanation for the higher bond strengths is greater timber penetration due to the low viscosity and longer cure time of the resin when compared to the other systems. Aquawrap® displayed lower bond strengths which are attributed to a lack of a primer and rigidity of the wraps. The phenolic systems did not show increases in capacity despite the increase in wrap length. None of the bond strengths were adequate to achieve the capacity of a new timber pile, although the 12 " Fyfe bond was close to the pile capacity. Pull-off testing revealed that systems with high shear bond strengths also displayed high tensile strengths while systems with low shear strength displayed low tensile strengths. Because of the correlations here, field tensile tests will likely reflect the condition of bond shear strengths of installed systems.

\subsection{Compressive Strength Evaluations}

The more layers of wrap utilized was directly proportional to the compressive strength resistance offered by any one of these systems that has been tested here in. The Fyfe and Sika systems provided the highest capacities with failure in the fibers. The Phenolic system also failed in the fibers, and the strength is attributed primarily to a lower longitudinal fiber density, although the 3 layer samples may have had insufficient thicknesses to achieve their expected strengths. Aquawrap experienced debond between wrap layers and consequently had the lowest strengths. 
Five layers of Fyfe and Sika systems should be able to provide enough capacity for the wraps while more evaluation is needed to determine the number of layers for the other systems. Compressive strength results are shown in Error! Reference source not found..

Table 5-1: Average compressive strength by number of wraps (psi)

\begin{tabular}{|c|r|r|}
\hline System & $\begin{array}{c}\text { 3 } \\
\text { Wraps }\end{array}$ & $\begin{array}{c}\text { 5 } \\
\text { Wraps }\end{array}$ \\
\hline Fyfe & 14,511 & 15,551 \\
\hline Sika & 13,809 & 14,915 \\
\hline Aquawrap ${ }^{\circledR}$ & 3,018 & 5,243 \\
\hline Phenolic & 5,152 & 7,660 \\
\hline
\end{tabular}

\subsection{Recommendations}

From the evaluations, the following recommendations are suggested:

- Fyfe and Sika wraps could be used to repair timbers to replace the capacity of a full pile.

- For higher capacities, more wraps should be applied with longer bond lengths after determining the strengths through additional testing.

\subsection{Future Work}

To further the significance of this study, the following additional work is recommended:

- Conduct more iterations of the bond push-out tests to determine effective methods for increasing the bond capacity such as different primers or trying vacuum bagging instead of stricture.

- Conduct additional pull off testing with the systems with varying timber substrates to further establish effective pull off bond strengths 
- Conduct with rapid moisture cycling evaluations on the bond systems to establish knock down factors from aging.

- Conduct compression evaluation on compression shells with a controlled eccentricity to determine an accurate estimation of its influence on final compressive strengths.

- Use a higher density fabric or additional layers for the phenolic system to determine if compressive strengths for this system can be increased.

- Conduct evaluations on filler materials to be used in tandem with the wrap systems.

- Conduct another round of tests on the Aquawrap® system utilizing a different installation method and with primers. 


\section{REFERENCES}

AASHTO, Maintenance Manual for Roadways and Bridges, 4th ed., 2007.

[2]

D. Breyer, K. Cobeen, K. J. Fridley and D. G. Pollock, "Chapter 4: Properties of Wood and Lumber Grades," in Design of Wood Structures - ASD/LRFD, 7th ed., 2015, pp. 186-187. the Effects of Moisture on Wood and Wood Products," in MNL18, Moisture Control in Buildings: The Key Factor in Mold Prevention, 2nd ed., 2009, pp. 54-78.

R. M. Rowell, "Moisture Properties," in Handbook of Wood Chemistry and Wood Composites, R. Rowell, Ed., CRC Press, Taylor \& Francis Group, 2005, pp. $77-98$.

M. A. Ritter, Timber Bridges: Design, Construction, Inspection, and Maintenance, Washington, DC.: U. S. Department of Agriculture, 1990.

R. N. Emerson, "In Situ Repair Technique for Decayed Timber Piles," in Structures 2004: Building on the Past, Securing the Future, 2004. 600/AFJPAM 32-1088)," Joint Departments of the Army and Air, Washington, DC, 1994. and Evaluation of Timber Piles Used in Nebraska for Retrofit and Rating," Miami, FL, 2014. 
F. W. Klaiber, D. J. White, T. J. Wipf, M. Mekkawy and J. Koskie, "Investigation of Steel-Stringer Bridges: Superstructures and Substructures, Volume II: Final Report," Bridge Engineering Center, 2007. Appropriate Maintenance, Repair and Rehabilitation Methods for Iowa Bridges," Iowa Department of Transportation, 2003. Milwaukee, WI, 2009.

P. Caiza, M. Shin and B. Andrawas, "Load Rating and Retrofit Testing of Bridge Timber Piles Subjected to Eccentric Loading: FHWA-ICT-12-014," Illinois Center for Transportation, November 2012.

Piling and Back Wall Rehabilitation and Repair," Bridge Engineering Center, 2012.

M. W. Hagos, "Repair of Heavily Decayed Timber Piles Using Glass Fiber Reinforced Polymers (GFRP) and Cementious Grout," Winnipeg, Canda, 2001.

R. Liang and H. V. S. GangaRao, "Fiber-Reinforced Polymer (FRP) composites in environmental engineering applications," in Developments in Fiberreinforced polymer (FRP) Composites for Civil Engineering, N. Uddin, Ed., Woodhead Publishing, 2013, pp. 410-468. Column Confined with Carbon Fibers Using Inorganic Matrix," Journal of Materials in Civil Engineering, vol. 19, no. 2, pp. 198-204, 1 Feb 2007. 
Fiber Wrap Composites for Timber Piles Repair and Protection of a Pier Structure," Coastal Engineering Practice, pp. 10-14, 2011.

Sika Coporation, "NYC- Pier Rehab," [Online]. Available: usa.sika.com/dms/getdocument.get/4b313d9e.../cs-cpd-NYC-Pier\%20Rehabus.pdf.

Air Logistics Coproration, "Timber Pile Repair," [Online]. Available: http://www.airlog.com/FACS/FACS\%20Aquawrap\%20Wood\%20Piling.htm.

B. King and H. GangaRao, "Rehabilitation of Timber Railroad Bridges using Glass Composite Fabrics," in Repairing and Rehabilitating the Buildings and Bridges of the Americas-Hemispheric Workshop on Future Direction, Mayguëz, Puerto Rico, 2001.

[21] A. Smith, "Rehabilitation of Timber Railroad Bridges Using Glass Fiber Reinforced Polymer Composite Wraps," Morgantown, WV, 2004.

[22] P. Vijay, G. Hota, R. Liang and M. Skidmore, "Rapid Restoration of Rail Road Timber Bridges Using Polymer Composites," in ANTEC, 2011. E. J. Barbero, "Chapter 4: Manufacturing Processes," in Introduction to Composite Materials Design, CRC Press, Taylor \& Francis Group, 2011, pp. 72-75. ACI Committee 440, "ACI 440.2R-08 Guide for the Design and Construction of Externally Bonded FRP Systems for Strengthening Concrete Structures," American Concrete Institute, July 2008. 
AASHTO, "Guide Specifications for Design of Bonded FRP Systems for Repair and Strengthening of Concrete Bridge Elements," 2012.

[26] AASHTO, AASHTO LRFD Bridge Design Specifications, U.S Customary Units with 2015 Interim Revisions, 7th ed., 2015.

[27] ASTM International, "ASTM Standard D25: Standard Specification for Round Timber Piles," West Conshohocken, PA.

[28] ASTM International, "ASTM Standard D2899: Standard Practice for Establishing Allowable Stresses for Round Timber Piles," West Conshohocken, PA.

[29] J. G. Collin, Timber Pile Design and Construction Manual, Timber Pile Counsel, 2002.

[30] G. Davis, "The Performance of Adhesive Systems for Structural Timbers," International Journal of Adhesion and Adhesives, vol. 17, no. 3, pp. 247-255, 1997.

$$
\text { C. Vick, "Adhesive Bonding of Wood Material," in Wood handbook - Wood }
$$
as an engineering material. Gen. Tech. Rep. FPL-GRT-113., US Department of Agriculture, Forest Service, Forest Products Laboratory, 2009. D. J. Gardner, J. F. Davalos and U. A. Munipalle, "Adhesive bonding of pultruded fiber-reinforced plastic to wood," Forest Products Journal, vol. 44, no. 5, pp. 62-66, May 1994. R. S. Abihari, "Rehabilitation of Timber Railroad Bridges Using Glass Fiber Reinforced Polymer Composites," Morgantown, WV, 2007. 
J. S. Lyons and M. R. Ahmed, "Factors Affecting the Bond between Polymer Composites and Wood," Journal of Reinforced Plastics and Composites, vol. 24, no. 4, pp. 405-412, 4 November 2005. D. Talakanti, "Testing and Evaluation of Wood-GFRC Adhesive Interface Integrity under Accelerated Aging and Mechanical Fatigue," 1997. C. Vick, "Coupling agent improves durability of PRF bonds to CCA-Treated Southern Pine," Forest Products Journal, vol. 45, no. 3, pp. 78-84, 1995. C. B. Vick, "Hydroxymethylated Resorcinol Coupling Agent for Enhanced Adhesion of Epoxy and Other Thermosetting Adhesives to Wood," in Wood Adhesive, Madison, WI, Forest Products Society, 1996, pp. 47-55. R. Lopez-Anido, A. P. Michael and T. C. Sandfor, "Fiber Reinforced Polymer

Composite - Wood Pile Interface Characterization by Push-Out Tests," Journal of Composites for Construction, vol. 8, no. 4, pp. 360-368, July/August 2004. ASTM International, "ASTM Standard D7522: Standard Test Method for Pull-Off Strength for FRP Bonded to Concrete Substrate," West Conshohocken, PA, 2012.

M. Saafi and E. Asa, "Extending the Service Life of Electric Distribution and Transmission Wooden Poles Using a Wet Layup FRP Composite Strengthening System," Journal of Performance of Constructed Facilities, vol. 24, no. 4, pp. 409416, August 2010. S. M. Petro, H. V. S. GangaRao, U. B. Halabe, S. Aluri, A. W. Smith, B. King, W. E. Steele and A. Vasudevan, "WVDOH RP\# 173: CARBON FIBER 


\section{REINFORCED POLYMER COMPOSITES USED TO REPAIR AND}

REHABILITATE WOOD RAILROAD BRIDGES," WVU-CFC, June 2007.

[42] X. Song, H. Tang, W. Zhang and X. Gu, "Compressive Stress Strain Relationship of Wood confined with Fiber Composite Sheets," Advanced Materials Research, Vols. 133-134, pp. 1207-1211, 2010.

[43] Timber Piling Council (American, Timber Pile Design and Construction Manual, American Wood Preservers Institute, 2002. 


\section{APPENDIX A - Bond Test Development}

For the bond testing, three different factors were evaluated: general influence of bond length, influence of surface smoothness, and influence of vacuum bagging versus stricture wrapping. The first two iterations used vacuum bagging to secure the wraps for bond testing.

For the first iterations, the focus was mainly on determining the effectiveness of the test methods. For the evaluations 4", 8", and 10" bond lengths were used as shown in Figure 5-1. From the initial testing no dramatic increase in strengths were observed and the longer specimens failed in crushing of composite before bond failure. From the results it was difficult to fully distinguish a noticeable increase in capacity as shown in Figure 5-2. Despite the limited results, the tests proved effective for their purpose.

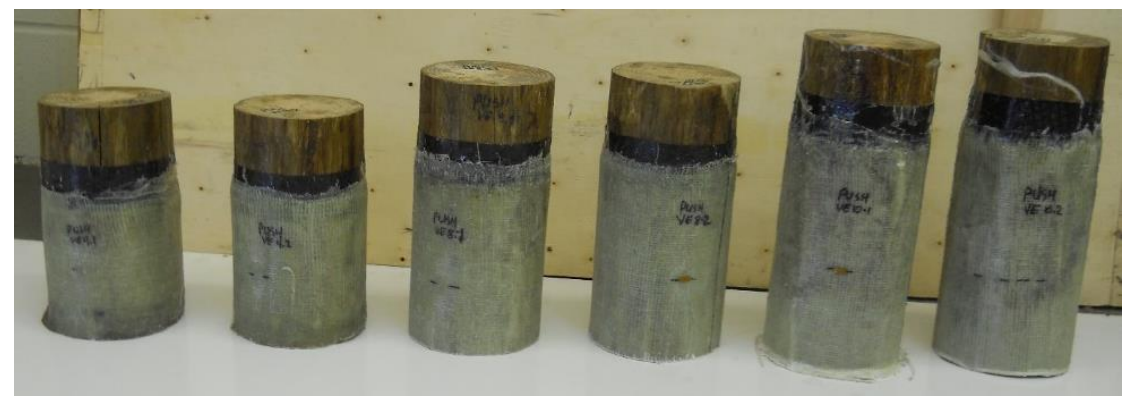

Figure 5-1: Preliminary evaluations of bond length with 4", 8" and 10" bond lengths

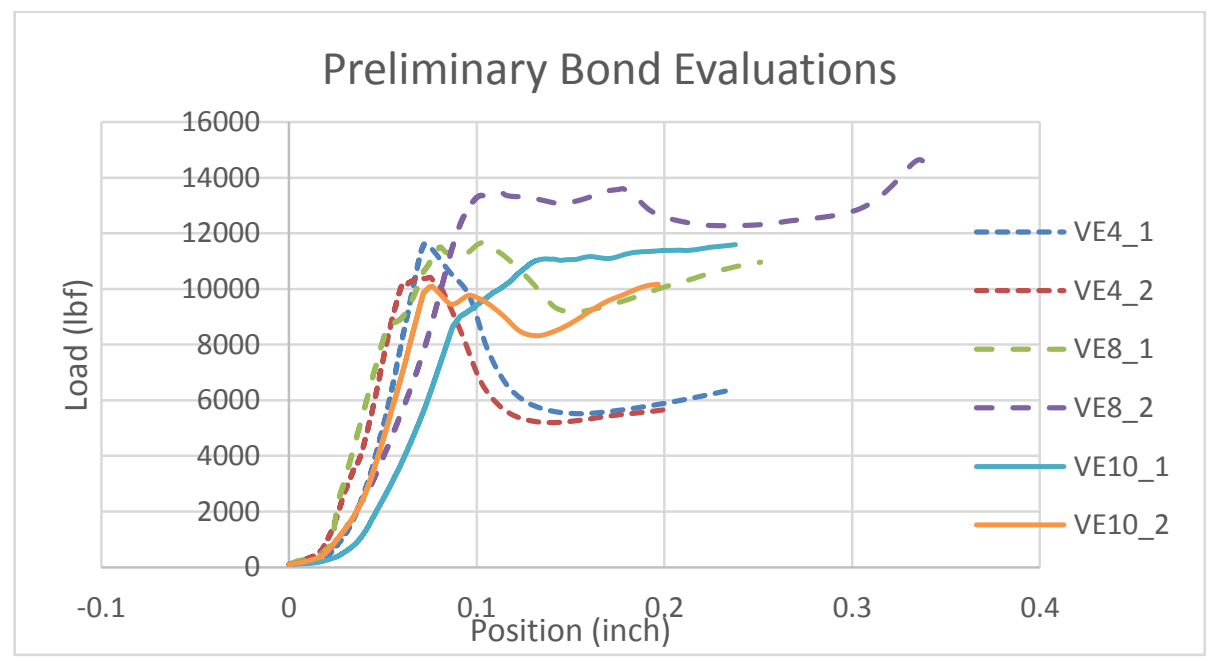

Figure 5-2: Preliminary evaluations with 4", 8", and 10" bond lengths 
For the second iteration, perfectly rounded specimens were created by lathing down timber to remove all surface defects possible. These next specimens were compared against those of the same bond length on non-perfectly smooth piles. The specimens are shown in Figure 5-3, those on the left are perfectly rounded while those on the right were unaltered. From the results (shown in Figure 5-4) it appeared that perfectly smooth surfaces actually had slightly lowered capacities. Results indicated that slightly rougher surfaces created a better bond then perfectly smooth surfaces.

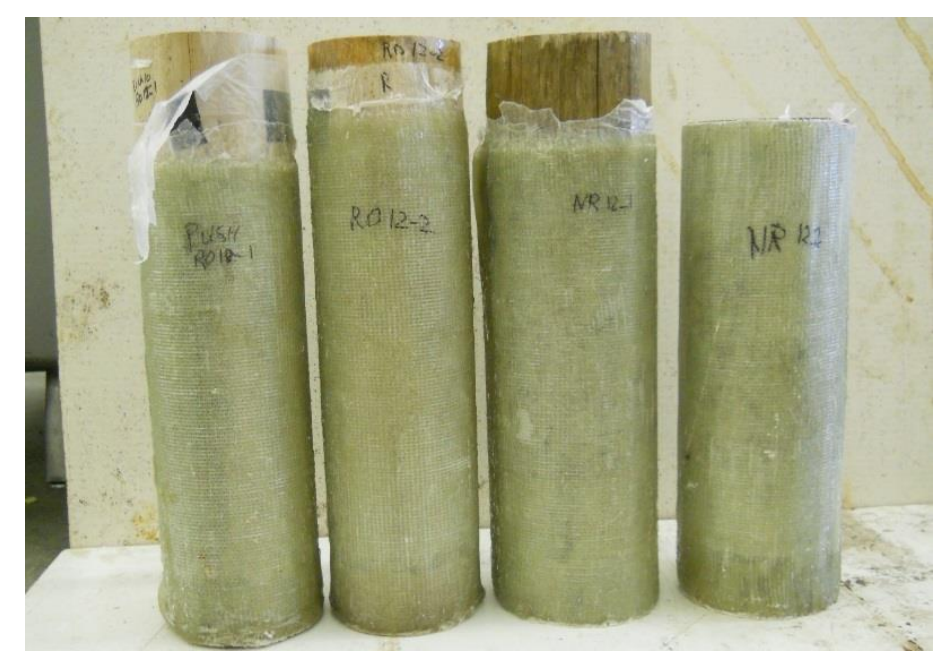

Figure 5-3: Comparing perfectly smooth and non-smooth surfaces

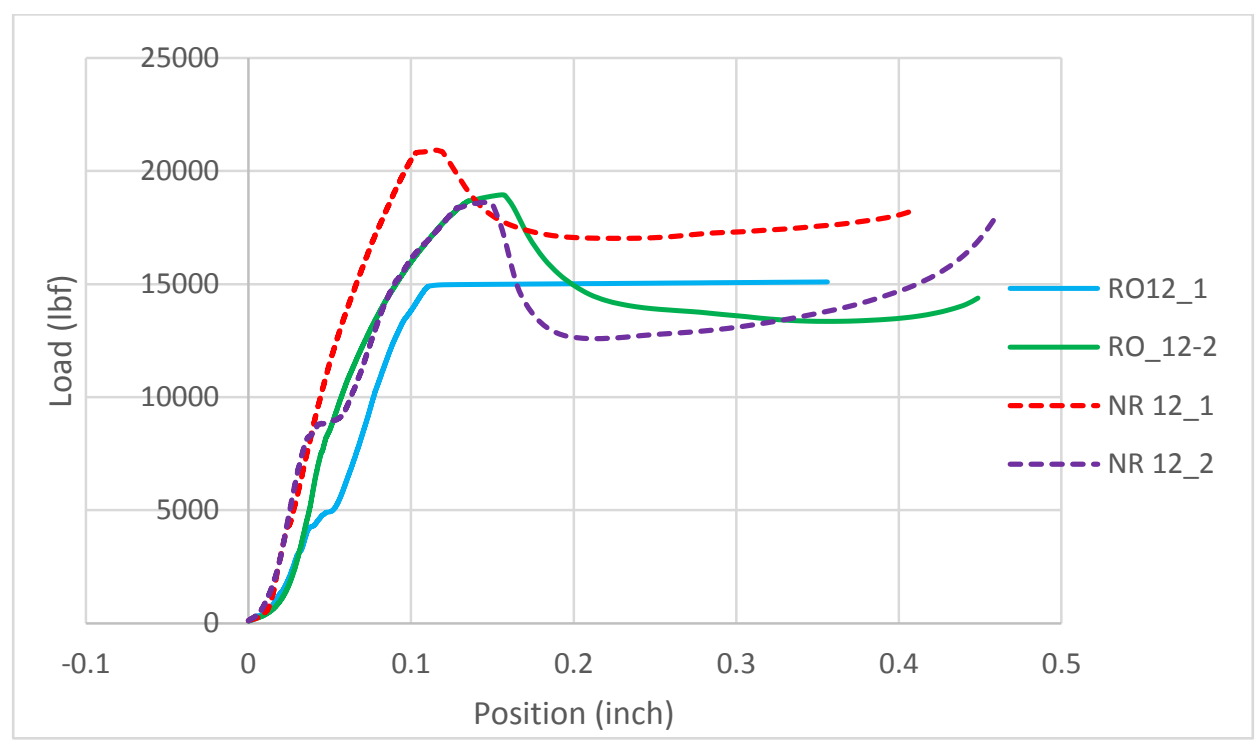

Figure 5-4: Perfectly round versus non-round results 
For the third iteration, vacuum bagging was compared against stricture wrapping as a pressure application process. Specimens A, C, and E used stricture wraps and specimens B, D, F used vacuum bagging as shown in Figure 5-5. The vacuuming bagging displayed slightly higher capacities then the stricture wrapping, but not by a noticeable amount as shown in Figure 5-6. Therefore stricture wrapping was considered to be viable alternative.
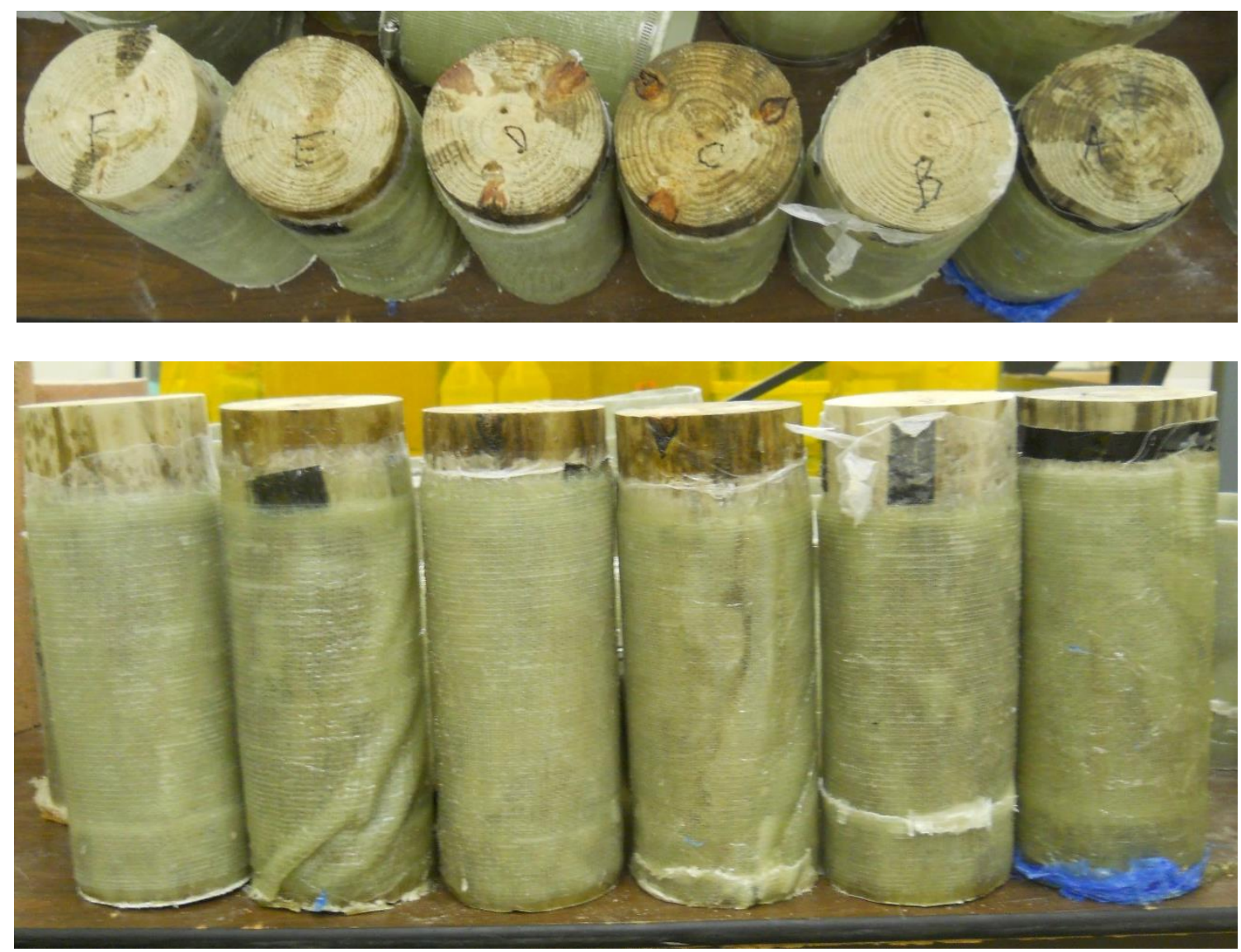

Figure 5-5: Preliminary evaluations with stricture wrapping and vacuum bagging 


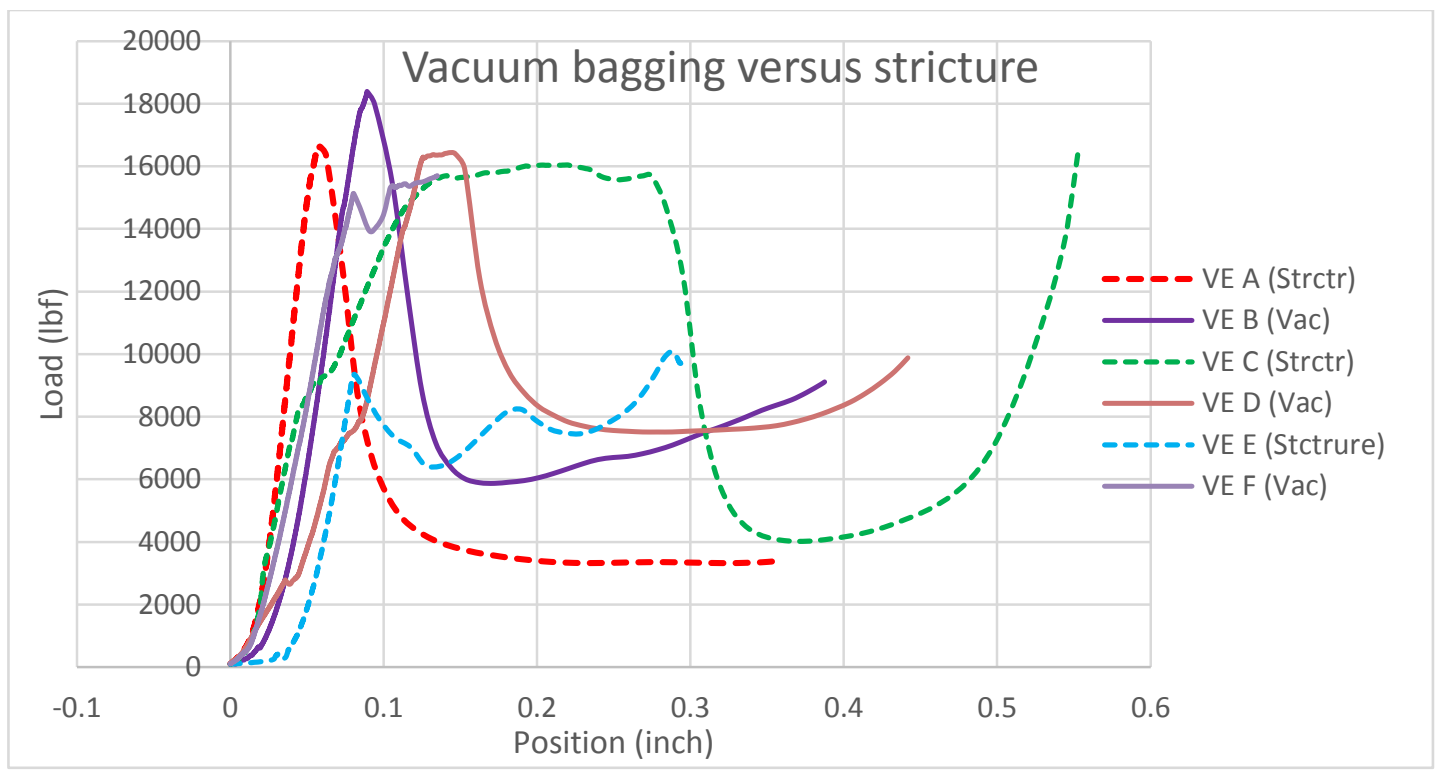

Figure 5-6: Vacuum bagging versus stricture wrapping 


\section{APPENDIX B - Compression Test Development}

To determine the most effective means of preventing fiber crushing several design iterations were attempted. Since the focus of the evaluations was simply to prevent fiber rushing in the base, no strength values are included.

First, evaluations were conducted on VE shells using high strength adhesive to attached 1" timber sections on the base to see if it could improve on the crushing issue. The first sample failed in compression while the second two failed in fiber crushing in the base with the behavior shown in Figure 5-7. Such failure indicated that the enhancements did not provide enough strengthening against crushing, requiring another iteration.
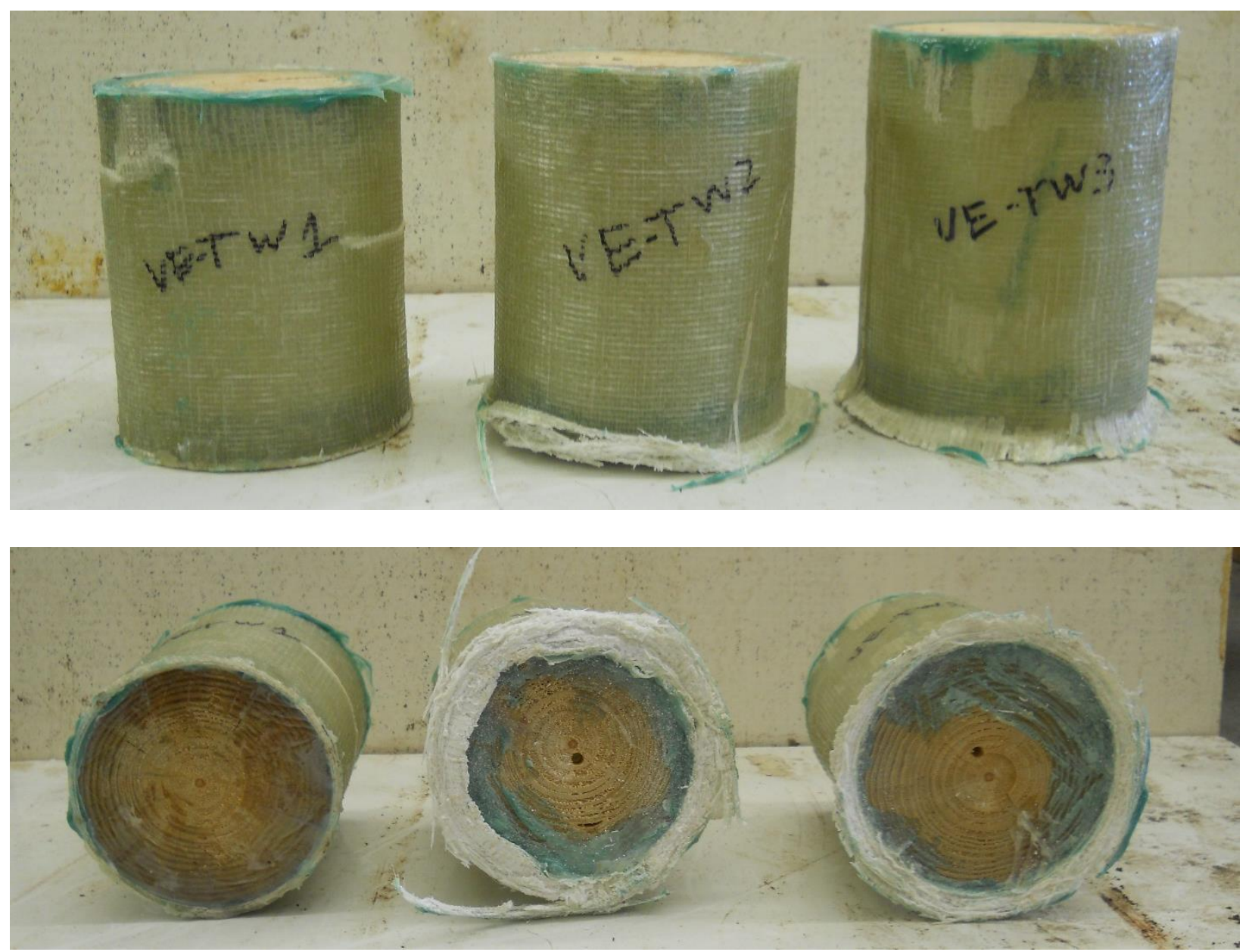

Figure 5-7: Crushing reinforcement using timber sections and adhesive 
For the second round of evaluations, PVC sections were secured with high strength adhesive with additional pipe clamp applied at the base of the specimens. All of the specimens failed in fiber crushing indicating that the reinforcement did not provide adequate protection against fiber crushing as shown in Figure 5-8.

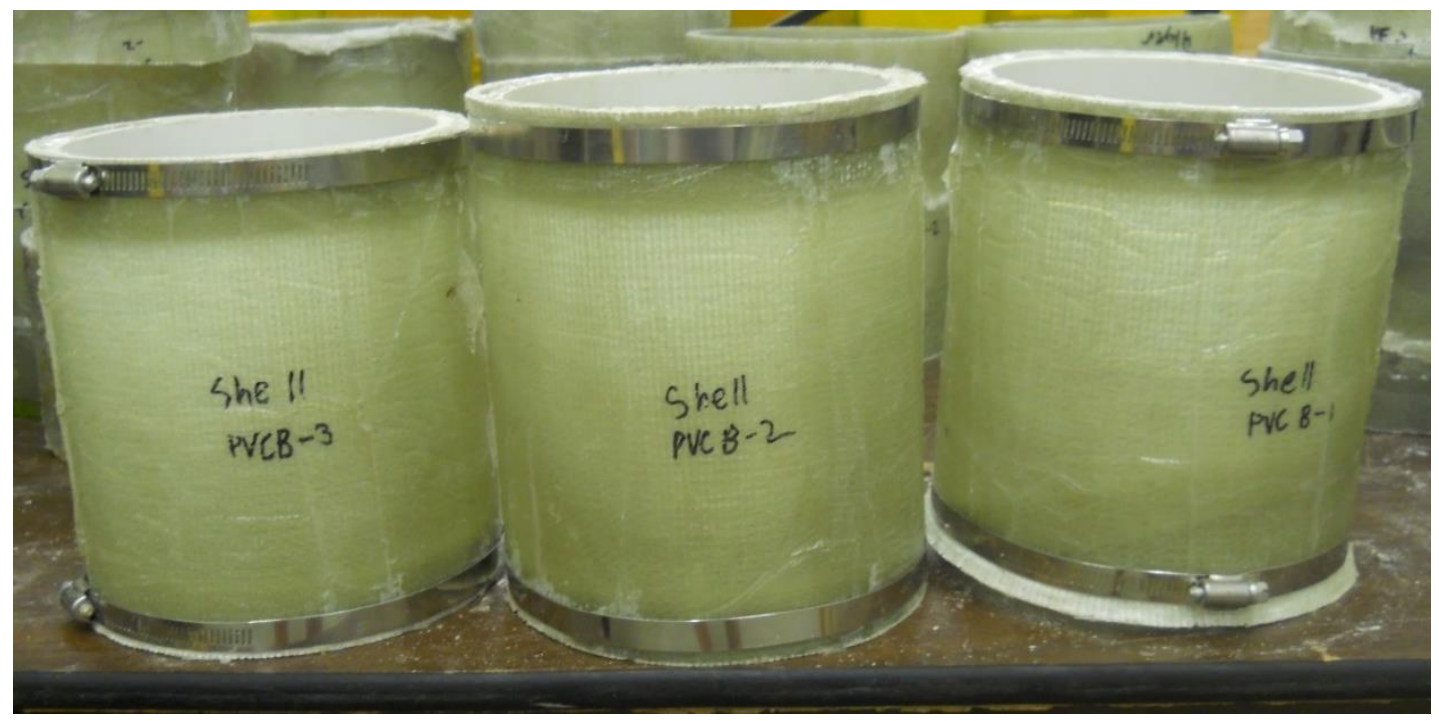

Figure 5-8: Crushing reinforcement using PVC and pipe clamps

For the third round of evaluations, three additional layers of wrap were applied. From these evaluations, it was found that additional layers of wrap at the based was sufficeint to prevent the crushing behavior as shown in Figure 5-9.

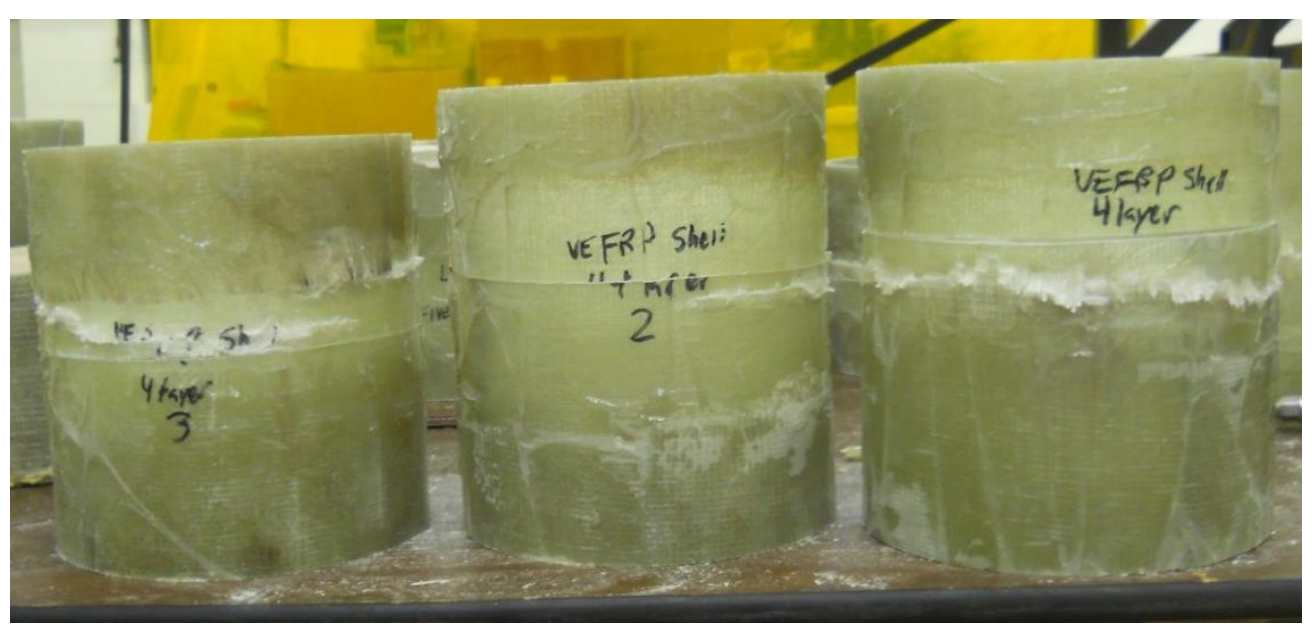

Figure 5-9: Crushing reinforcement using three additional layers of wraps 